Copyright

By

Hangwen Li

2010 
The Dissertation Committee for Hangwen Li certifies

that this is the approved version of the following dissertation:

\section{Prostate Cancer Stem Cells and Their Involvement in Metastasis}

\section{Committee:}

Dean G, Tang Supervisor

Christopher A Jolly Co-Supervisor

Sue Fischer

Michael MacLeod

Kimberly Kline

Bob Sanders 


\title{
Prostate Cancer Stem Cells and Their Involvement in Metastasis
}

by

HANGWEN LI, B. Med

\author{
DISSERTATION \\ Presented to the Faculty of the Graduate School of \\ the University of Texas at Austin \\ in Partial Fulfillment \\ of the Requirements \\ for the Degree of
}

\section{DOCTOR OF PHILOSOPHY}

\section{THE UNIVERSITY OF TEXAS AT AUSTIN}

August 2010 


\section{Dedication}

To my parents, who have always been behind me and supported me, and have given me the principles for being a man

To my wife, Xiran, who gives me the sweetest love and happiness in the world and has encouraged me to overcome all the difficulties we have encountered 


\section{Acknowledgements}

It is really an exciting time to be close to finishing my Ph. D journey, not only because of the 7 years I have spent on the road, but also the scientific training, honor and perseverance I have gained. I could not have completed all of these without one person's guide. This is my mentor, Dr. Dean Tang. I would like to express my deepest gratitude to him, for his knowledge, passion and dedication to science. I have learnt a lot from him both professionally and personally that will stay with me for the rest of my life. A special thank also goes to my co-mentor, Dr. Christopher Jolly. He brought me to the US and helped me to start my career. I also would like to express my appreciation to my committee members from both UT Nutrition and M.D. Anderson Science Park: Dr. Sue Fischer, Dr. Michael MacLeod, Dr. Kimberly Kline, and Dr. Bob Sanders who have given me constructive advice and supervision. The big Tang family always provides an exciting work environment and I have really enjoyed working here. In particular, I would like to thank Dr. Mahipal Suraneni and John Moore for their valuable insight and collaboration; Dr. Wei Zhang and Dr. Da Yang from department of pathology, MD. Anderson Cancer center for their fantastic bioinformation contribution; Mark Badeaux for his great help in my writing, his genius humor, and organizing entertainment and sport activities; Tammy Calhoun-Davis for her help for the mouse work. I also thank all my Chinese colleagues: Dr. Jichao Qin, Xin Chen, Xin Liu, Can Liu, Bigang Liu, Dr. Hong Yan, for their tremendous help and for making me less homesick. I thank Dr. Hongxin Lu from UT for her invaluable suggestions. I also would thank the DOD PCRP 
for a prestigious pre-doctoral fellowship. Finally, I would like to thank the Flow Cytometry, Molecular Biology, Histology Cores, as well as the Animal Facility Core in Science Park for their great support. 


\title{
Prostate Cancer Stem Cells and Their Involvement in Metastasis
}

\author{
Publication No.
}

\author{
Hangwen Li, Ph.D. \\ The University of Texas at Austin, 2010
}

\author{
Supervisor: Dean G. Tang \\ Co-supervisor: Christopher A Jolly
}

The recently resurrected cancer stem cell (CSC) theory sheds new light on understanding tumor biology. Most solid tumors have now been shown to contain CSCs, i.e., stem cell-like cancer cells. These cells, although generally rare, appear to be highly tumorigenic and may be the cells that drive tumor formation, maintain tumor homeostasis, and mediate tumor metastasis. In order to test whether any given human tumor cell population has CSC properties, the relatively enriched single tumor cells have to be put into a foreign microenvironment in a recipient animal to test their tumorigenic potential. Furthermore, various in vitro assays can be performed to demonstrate that the presumed CSCs have certain biological properties normally associated with the stem cells (SCs). Herein, I first present a comprehensive review of the experimental methodologies 
that our lab has been using in assaying putative prostate cancer (PCa) SCs in culture, xenograft tumors, and primary tumor samples.

Clonal morphology is one of the critical properties of cultured cancer cells that has been largely ignored. Interestingly, long term-cultured human epithelial cancer cells form holoclones, meroclones, and paraclones, and tumor cell holoclones have been hypothesized to harbor stem-like cells. Using PC3 human prostate carcinoma cells as a model, we provide direct experimental evidence that tumor cell holoclones contain stemlike cells that can initiate serially transplantable tumors. Importantly, holoclones derived from either cultured PC3 cells or holoclone-initiated tumors can be serially passaged and regenerate all three types of clones. In contrast, meroclones and paraclones cannot be continuously propagated and fail to initiate tumor development. Phenotypic characterizations reveal high levels of $\mathrm{CD} 44$, a $2 \beta 1$ integrin, and $\beta$-catenin expression in holoclones, whereas meroclones and paraclones show markedly reduced expression of these markers. These observations have important implications in understanding morphologic heterogeneities and tumorigenic hierarchies in human epithelial cancer cells.

PCa metastasis represents the worst outcome, and, if unchecked, will eventually kill the patient. Although many PCa cell-intrinsic molecules and end-organ factors have been implicated in the metastatic dissemination of PCa cells, the role of primary tumor microenvironment and the nature of the metastatic PCa cells remain poorly defined. By establishing a reliable and quantifiable experimental PCa metastasis model in NOD/SCID mice, we show that PCa cells implanted orthotopically (i.e., in the prostate) metastasize much more extensively and widely than those implanted ectopically (i.e., subcutaneously 
or s.c). Microarray-based gene expression profiling reveals that the orthotopically implanted human PCa cells prominently overexpress not only several classes of molecules involved in proteolysis/invasion/angiogenesis and inflammation, but also numerous developmental and SC regulating genes. These latter observations suggest that the orthotopic microenvironment (i.e. mouse prostate) appears to be promoting the manifestation of CSC phenotypes and these CSCs might be involved in enhanced metastasis in the orthotopic microenvironment and later distant organ metastasis.

In support, shRNA-mediated knockdown in many metastatic and CSC genes greatly inhibits PCa cell metastasis. Importantly, PCa cells that express high levels of osteopontin (OPN) or CD24, when prospectively purified out and used in spontaneous metastasis assays, demonstrate high metastatic capacities characteristic of metastatic CSCs. In sharp contrast, PCa cells negative for OPN and CD24 expression show little metastatic property. Finally, we provide multiple pieces of additional evidence that metastatic/metastasizing PCa cells possess CSC properties. 


\section{TABLE OF CONTENTS}

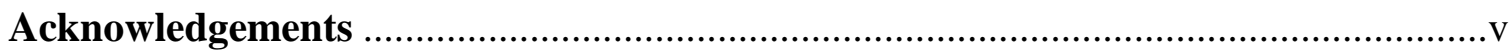

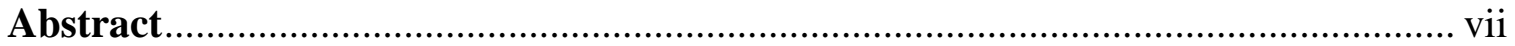

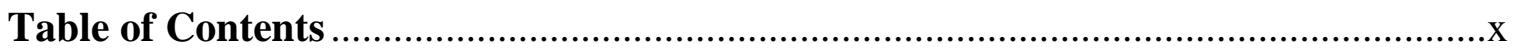

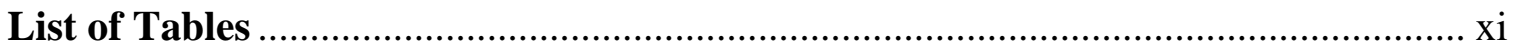

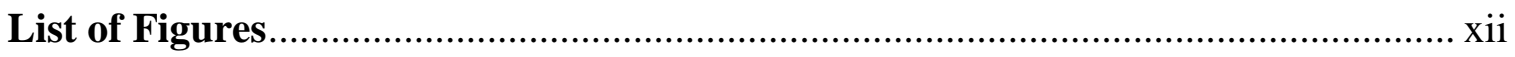

Chapter I. Introduction

Chapter II. Assays for identification of Prostate Cancer Stem Cells .............................22

Chapter III. PC3 Human Prostate Carcinoma Cell Derived from Holoclones Contain Self-Renewing Tumor-Initiating Cells .................................................4 41

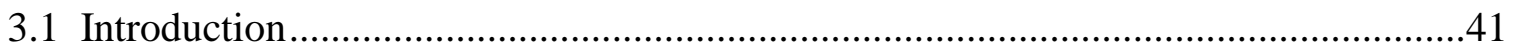

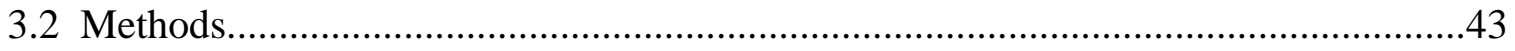

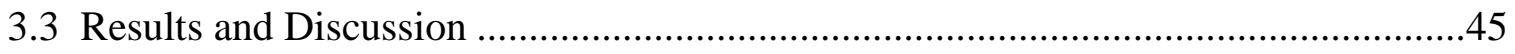

Chapter IV. Microenvironment-Regulated Manifestation of Metastatic Prostate Cancer Stem Cells ...........................................................................58

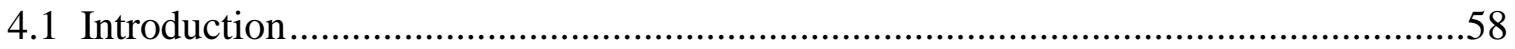

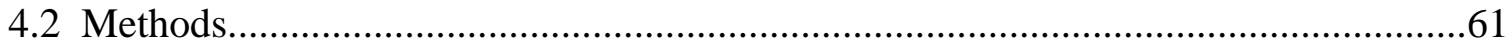

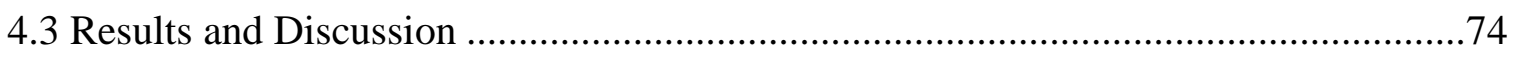

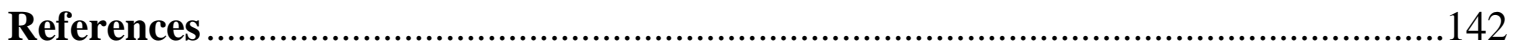

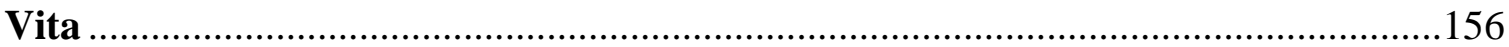




\section{LIST OF TABLES}

2.1 CSC Studies in Human Solid Tumor (2003-2008) ..............................................23

2.2 Variables affecting the outcome of human prostate cancer tissue/cell

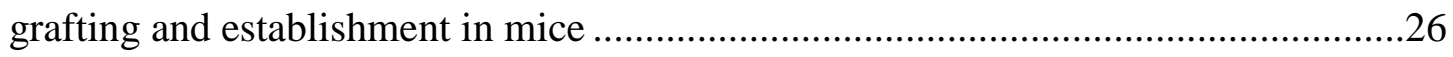

2.3 Mouse strains commonly used in CSC studies .................................................27

3.1 Tumorigenicity and serial transplantability of PC3-GFP cell clones ........................52

4.1 Tumor-initiating frequency (TIF) of PCa cells subcutaneously (s.c)

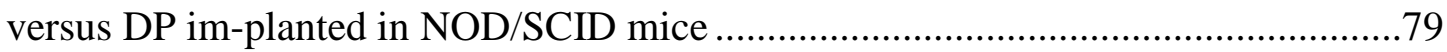

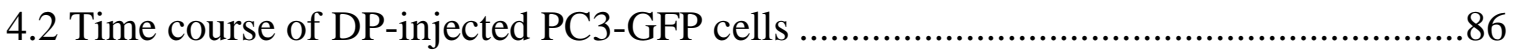

4.3 Representative human genes commonly upregulated in DP versus s.c

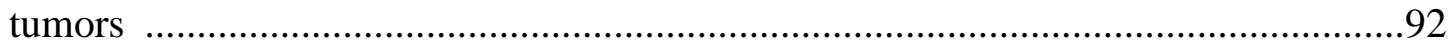

4.4 Representative mouse genes commonly upregulated in $3 \mathrm{DP} / \mathrm{SC}$ tumor

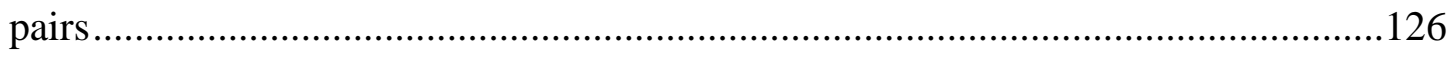

4.5 Summary of prediction result based on three signatures ...................................... 137 


\section{LIST OF FIGURES}

1.1 Killing the root of cancer

1.2 CSC related oncogenic pathways involved in growth, survival, invasion and drug resistance

1.3 Two hypothetical models explaining origin of human breast cancer heterogeneity.

1.4 A modified integrative model of breast cancer metastasis 20

2.1 A model of hierarchical organization of tumorigenic PCa cells 30

2.2 SP analysis of LAPC9 tumor cells on two different flow cytometers

2.3 Comparison of tumor development of prostate CSC populations (i.e., the SP and CD44+ cells acutely purified from the GFP-tagged LAPC9 tumors) implanted at different sites

2.4 The s.c. site is also the most sensitive microenvironment to reconstitute tumordevelopment from pieces or cells of the primary $\mathrm{PCa}$ samples. .39

3.1 Cultured PCa cells exhibit three distinct clonal morphologies .46

3.2 Clonal Heterogeneity of PC3 Cell .48

3.3 Developmental fate of three types of clones during the 12-wk followup period

3.4 Both P1E2 holoclone-derived cells (A) and P1E2 tumor-derived cells (B)

3.5 Serial tumor transplantation of three holoclone-derived PC3 cells .53 
3.6 PC3 cell holoclones express high levels of stem and progenitor cell markers

4.1 Different metastatic potential of PC3 cells implanted in the prostate vs s.c...... .76

4.2 Differential Lung metastasis between DP and sc implanted cells ( $10,000)$ .78

4.3 Human PCa cells implanted into orthotopic sites demonstrate less primary tumor growth and more widespread metastasis than cells implanted in the ectopic sites .80

4.4 The AP-implanted PCa-GFP cells metastasize to multiple organs

4.5 HPCa-80 Tumor growth and metastasis profiles in two implantation site DP vs sc

4.6 Schematic chart for gene microarray and clustering analysis upregulated human genes...

4.7 Pie graph showed the Gene Ontology analysis of DP up-regulated human genes

4.8 Verification of gene expression identified by microarray . .94

4.9 OPN WB in LAPC9 DP/sc tumor samples .95

4.10 shRNA knockdown tumor experiments in PC3 model. .99

4.11 MMP9 is functionally involved in PCa cell invasion and metastasis 100

4.12 PC3-GIPZ-OPN2 Knockdown Xenograft model suppress metastasis 101

4.13 OPN expression in the cell surface of PCa. 104 
4.14 OPN+ cells exhibit strong tumor growth and metastasis potential compared with OPN- counterpart

4.15 CD24 expression in the cell surface of PCa cells by detection of flow

cytometry.

4.16 PC3-GFP CD24+ population enriched metastasis potential 109

4.17 LAPC9-AI-GFP-DP CD24 +/- tumor and metastasis....

4.18 Flow chart for serial enrichment PC3 Model

4.19 DP-implanted PC3-GFP cells at high cell numbers (i.e., 2,000,000

cells) metastasize to multiple organs

4.20 Second round Metastatic LM1 and BM1 enhance tumorigenesis and metastasis potential

4.21 CD24 and CXCR4 expression are increased in LM1 and BM1

4.22 SP enriched in BM1 cells 118

4.23 PC3-GIPZ-TGFß2 KnockDown suppressed metastasis 120

4.24 TGF- $\beta 2$ regulated stem cells markers OPN and CD44 expression

4.25 Ingenuity Pathway Analysis (IPA) of Up-regulated genes in DP vs sc com-parisons (human).

4.26 Commonly upregulated genes in both human and mouse. 128

4.27 Tumor host cross talk pathway. 129

$4.28 \alpha$-SMA/S100A9 expression in LAPC9 and PC3 DP vs s.c. 130

4.29 Reconstitute Metastasis profiles in mouse subcutis 
4.30 Genes whose down/up-regulated expression in metastasis validated

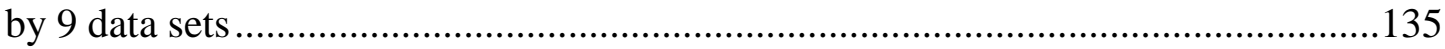

4.31 K-mean clustering identify high risk and low risk prostate cancer samples based on six down-regulated (A and B), 40 up-regulated genes $(\mathrm{C}$ and $\mathrm{D})$ and the stem cell markers $(\mathrm{E}$ and $\mathrm{F})$ 


\section{Chapter I: Introduction}

\section{The Lethal 'Seeds' of Cancer and Metastasis}

There is only one ultimate target of cancer research: to eliminate cancer. This means not only removing primary tumor or reducing the primary tumor burden, but also blocking recurrence and metastasis. Cancer could come back and metastasize after tumor therapy that usually targets the bulk of tumor cells (Rove and Crawford, 2009). The CSC hypothesis is a model that advocates the idea of eliminating cancer without recurrence and metastasis (Weissman, 2000). In this model, CSCs are the seeds of lethal cancer and metastasis if one gets rid of the seeds, the tumor will neither develop nor metastasize. Much like the seeds or roots of grass, if you want to kill wild grass, you must destroy or remove the seeds and roots; if you only damage the stem, grass will finally come back (Henderson et al., 2008; Hill and Wu, 2009)(Figure 1.1). The next critical and practical question then is how to find and destroy the seeds/roots of cancer. Cancer is a complicated process and disease, and unlike how you can easily distinguish seeds/roots from the stem of a grass based on the distinct morphology, it is generally difficult to distinguish seeds/roots from the bulk of tumor cells due to lack of defined markers.

In my Ph.D thesis research, my focus has been to identify the CSCs, the potential lethal seeds/roots of cancer and metastasis in PCa and metastasis. Since tumor is heterogeneous and made of different subpopulations, identification of CSCs is linked closely to understanding tumor heterogeneity, one of the key features of cancer (Shackleton et al., 2009).

\section{Tumor Heterogeneity}




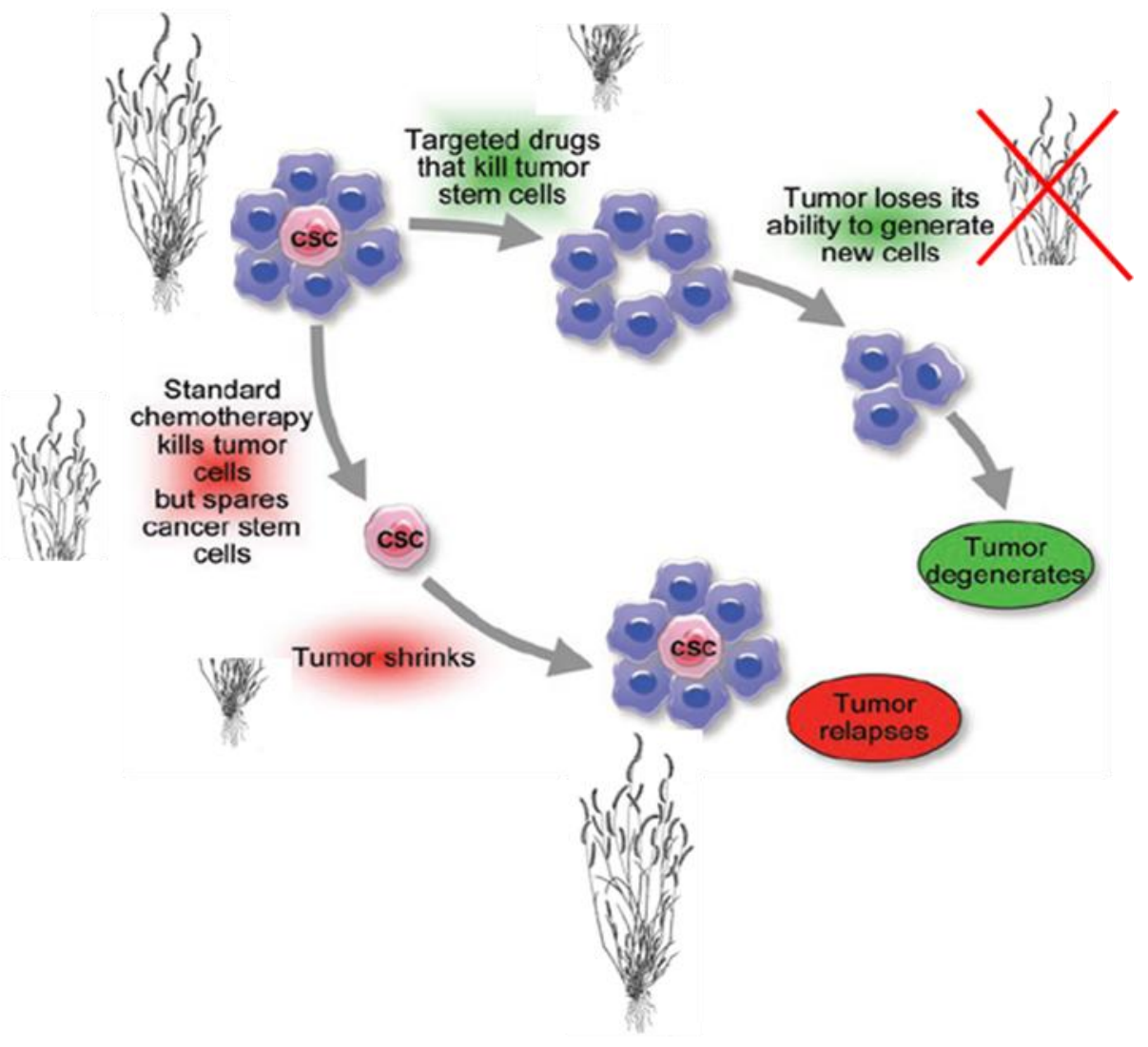

Figure 1.1. Killing the root of cancer

Only targeting the CSC, the root of cancer, can theoretically eliminate cancer successfully. Standard chemotherapy kills the bulk tumor cells, like the stem of grass. As a result, tumor will only shrink and relapse later (adapted from Hermann PC, et al., Cell Cycle. 2008) 
Tumor heterogeneity is a hallmark of cancer. The biological and clinical significance of cancer cell heterogeneity is likely linked to distinctions between tumorigenic and non-tumorigenic cells (Marusyk and Polyak, 2010). Cancer heterogeneity exists on two levels: between tumors and within a single tumor. Epithelial tumors or carcinomas could be classified to different subtypes by their different metastatic ability and stages (Campbell and Polyak, 2007). For example, PCa is thought of as a multistage process including sequential progression from normal tissue to hyperplasia, to prostatic intraepithelial neoplasia (PIN), and then to invasive and metastatic stages. Each subtype of tumor exhibited distinct diagnostic (with different Gleason grade) traits and prognosis, as well as unique gene expression (Man and Gardner, 2008). Within a single tumor, there also exists a striking variety of cell features including differences in cell size, morphology, cell surface markers and antigen expression, as well as cell behaviors like proliferation, metabolism, and metastatic potential etc. Cancer heterogeneity is influenced by genetic, epigenetic, and microenvironmental factors. The cause of cancer heterogeneity and molecular mechanisms that control this process are fundamental issues in understanding the causes of tumor development and tumor metastasis (Marusyk and Polyak, 2010). In addition, understanding these molecular pathways could lead to potential progress in cancer therapy. However, all of these problems remain poorly understood. The CSC hypothesis and clonal evolution model have been evoked to explain tumor heterogeneity (Campbell and Polyak, 2007; Sgroi, 2010).

\section{The CSC Hypothesis}


It was reported $>50$ years ago that a minor subset $(0.01 \%-1 \%)$ of cancer cells actually isolated from a tumor had the ability to regenerate a clonal growth or a tumor, suggesting that such rare cells might represent tumor stem cells (Reya et al., 2001). These CSC may be able to self-renew, have a distinct advantage of tumor regeneration, and produce the heterogeneous subsets of cancer cells that comprise the tumor (Clarke et al., 2006). The first direct evidence of CSCs came from John Dick's group on acute myeloid leukemia (AML) by using CD $34^{+} / \mathrm{CD}^{-} 38^{-}$as the marker profile (Bonnet and Dick 1997). Michael Clark's group was the first to report on CSCs in solid cancer by isolating $\mathrm{CD}_{4} 4^{+} / \mathrm{CD} 24^{-}$subpopulation in breast cancer (Al-Hajj et al., 2003). In the past decade, CSCs have been reported in multiple cancers (Li et al., 2009; Shackleton et al., 2009). In these studies, CSCs have been identified by using normal stem/progenitor cell markers. The gold standard for functionally defining CSCs is to test the enriched tumorigenic ability by serial transplantation to show CSC self-renewal ability in a xenograft mouse model. The newly generated tumors from the potential CSCs should contain the full repertoire of heterogeneity of cancer cells observed in the parental tumors (Tang et al., 2007). One limitation for current CSC studies is that they mainly utilize tumor initiation as the major yardstick to measure CSC properties without analyzing metastasis, multi-potency and asymmetric cell division. Consequently, "cancer initiating cells" may be more appropriate than the term CSCs.

\section{CSC Generate Tumor Heterogeneity}




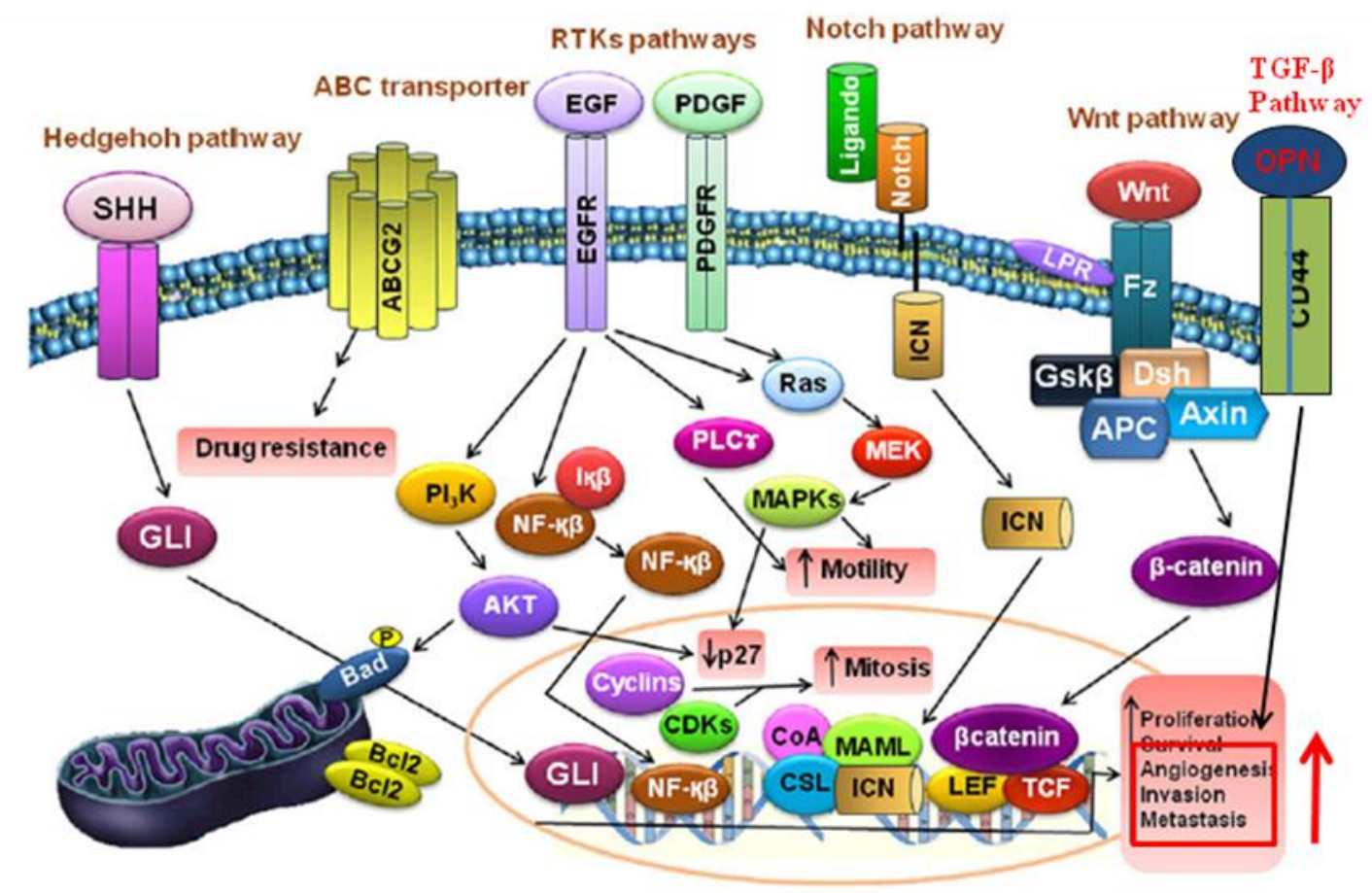

Figure. 1.2. CSC related oncogenic pathways involved in growth, survival, invasion and drug resistance

Except these established pathways such as Wnt, Notch, et al, TGF- $\beta$ may regulate CSC through OPN and CD44. These CSC pathways correlate with Angiogenesis/Invasion/metastasis, implying that metastasis is an important property of CSCs (adapted from Vera-Ramirez et al. Cancer Treatment Reviews 2010) 
There is ample evidence that demonstrates the link between normal SCs and CSCs. Most reported CSC markers are normal SC markers. Many of the normal SC markers and pathways, such as CD44, Wnt, Notch, Hedgehog, and BMI, are also highly up-regulated in malignant tumors and metastases (Vera-Ramirez et al., 2010) (Figure1.2). These 'stemness pathways' also correlate with metastasis. In addition, a recent study indicates that an embryonic SC gene signature correlates with tumor grade (Ben-Porath et al., 2008). Similarly, another group demonstrated that a normal breast tissue stem cells signature was enriched in highly malignant breast tumors (Pece et al., 2010). Therefore, a better appreciation of the CSC model and its significance in heterogeneity and tumor therapy largely relies on understanding normal SC biology. In normal tissue, only the SC holds the properties of unlimited proliferation. A SC passes this intrinsic 'immortal' trait to another SC and a daughter cell by asymmetric division, a process known as SC self-renewal (Shackleton et al., 2009; Weissman, 2000). The daughter cell, also called progenitor cell, has a more limited proliferative ability and lacks the self-renewal ability, and eventually will differentiate to a mature functional cell (Weissman, 2000). The process of $\mathrm{SC} \rightarrow$ progenitor $\rightarrow$ mature cell is normally under tight control, which creates the cellular hierarchy of normal tissue (Campbell and Polyak, 2007; Marusyk and Polyak, 2010; Shackleton et al., 2009). Similar to normal SCs, CSCs are hypothesized to be undifferentiated and able to self-renew and generate daughter cells that can differentiate into more mature cancer cells. In figure $1.3 \mathrm{~B}$, an example on breast cancer is given to illustrate the hypothesis of CSC generating tumor heterogeneity. In CSC based tumor therapy, when CSCs are eliminated, tumor development will be halted and tumor recurrence will not occur (Hermann et al., 2008). 


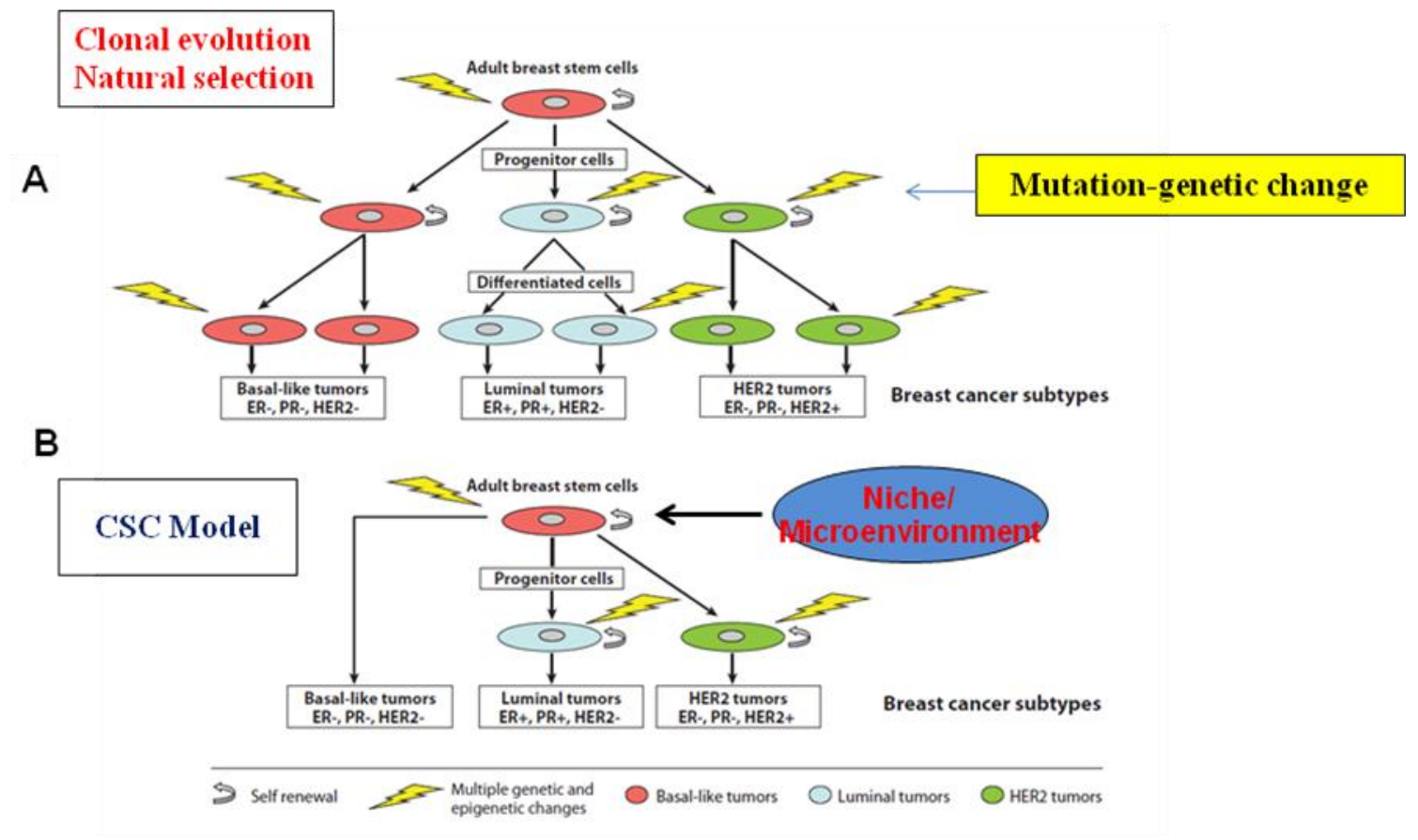

Figure 1.3. Two hypothetical models explaining origin of human breast cancer heterogeneity

(A) Clonal evolution model. The epithelial cells (e.g., stem cell, progenitor, or differentiated cell) may be the target of transformation to initiate breast cancer. Each breast cancer subtype with different origins forms breast cancer heterogeneity. (B) CSC model. The initiating events target the SCs or progenitor cells to initiate tumor development. CSCs are originated from normal SCs and can differentiate into progenitor cells and more mature differentiated cells under the influence of niche. One significant point between these two models is that clonal evolution mainly depends on intrinsic genetic changes to undergo natural selection whereas CSC tumor phenotype is determined by a combination of genetic and epigenetic events.

Abbreviations: $\mathrm{ER}+(-)$, presence(absence) of immunohistochemical expression of estrogen receptor (ER) expression; HER+(-), presence (absence) of immunohistological expression or HER gene amplification; PR+(-), presence (absence) of immunohistochemical expression of progesterone receptor $(\mathrm{PR})$ expression.

(adapted from Sgroi DC., et al., Annual Review of Pathology: Mechanisms of Disease. 2010) 
Traditional target of differentiated tumor cells can shrink the tumor size but any unaffected CSCs might re-initiate the tumor and cause recurrence and metastasis (Reya et al., 2001).

Compared with the well-defined hierarchy elucidated in some normal stem cells, our understanding of CSC-derived cellular heterogeneity in most tumors is very limited. These differences may be related to the significant genetic alternations in CSCs. The microenvironment and epigenome between normal SCs and CSCs are also dramatically different. An increasing number of studies have started to address such differences in the CSC field (Pece et al., 2010; Polyak et al., 2009). Some cancers like germ cell tumors and certain leukemia appear to follow a tumor hierarchy model in which some primitive cancer cells can differentiate into post-mitotic derivatives (Shackleton et al., 2009). A recent study using Numb as the marker in breast cancer demonstrates that breast CSCs can undergo asymmetric divisions (Pece et al., 2010). An ongoing study in our lab reveals that human prostate CSCs can also divide into one CSC and another nonCSC by asymmetric division (Qin et al, manuscript in preparation).

\section{Clonal Evolution and Tumor Heterogeneity}

Clonal evolution is another potential mechanism that could generate tumor cell heterogeneity (Campbell and Polyak, 2007). The biggest difference between the CSC hypothesis and clonal evolution lies in the principle of cancer therapy: in contrast to CSC hypothesis, clonal evolution based therapy targets all cells in a tumor; it does not focus on any special subpopulation inside the tumor. Nowell first proposed the clonal evolution model in 1976 since he observed that tumor progression was in a interesting pattern: different genetic variable clones with more aggressive properties were sequent selected to maximize their proliferation and invasiveness 
potential (Nowell, 1976). It implies that these individual clones may hold a growth advantage over other cells due to more genetic instability. The most significant point of the clonal evolution model is that cancer can initiate from any random single cell if it happens that this single cell has accumulated necessary mutations to drive malignancy. There is no difference between SCs and non-SCs in this model. The tumorigenic clones acquire the advantage of growth and malignancy by winning the competition against their adjacent cells to survive. During tumor procession, only the dominant clones proliferate well (e.g., resistance to apoptosis) and acquire additional mutations to promote increased invasiveness or resistance to therapies and recurrence. According to this model, tumor cells are always in different mutation stages and intrinsic genetic alterations gained from multiple mutations select tumor cells with unlimited proliferative potential. In other words, genetic diversifications result in tumor heterogeneity (Figure1.3 A)(Campbell and Polyak, 2007). This cancer evolution model is akin to Charles Darwin's "natural selection" for species evolution (Greaves, 2010) .

Clonal evolution is supported by several recent studies. In breast cancer, Polyak's group demonstrates a distinct molecular gene signature between $\mathrm{CD} 44^{+}$versus $\mathrm{CD} 24^{+}$subpopulations (Shipitsin et al., 2007). The $\mathrm{CD}^{+} 4^{+} / \mathrm{CD} 24^{-}$breast cancer cells have been reported previously as CSCs (Al-Hajj et al., 2003). In support, they show that $\mathrm{CD}_{4} 4^{+}$population's gene signature contained many known SC genes and correlated with decreased patient survival (Shipitsin et al., 2007). According to CSC hypothesis, the CD24 ${ }^{+}$breast cancer cells might be the progenitor cells or more matured progeny derived from the $\mathrm{CD} 44^{+} / \mathrm{CD} 24^{-}$population (Al-Hajj et al., 2003). Therefore, the genetic differences between the $\mathrm{CD} 44^{+}$and the $\mathrm{CD} 24^{+}$are expected to be minor. However, Shipitsin et al observed distinct genetic differences between these two populations, 
suggesting that $\mathrm{CD} 44^{+}$and $\mathrm{CD} 24^{+}$subpopulations are clonally distinct and not related to CSC lineage development (Shipitsin et al., 2007). In colon cancer, by selecting single primary cancer cell-derived clone, lineage markers for mature cells like Muc-2 and Villin were strongly expressed in only some tumor-initiating clones, implying that tumor initiate from a clone selection model (Odoux et al., 2008; Vermeulen et al., 2008). In addition, genetic studies on acute lymphoblastic leukemia (ALL) also reveal a complex, non-linear, clonal architecture with subclones having distinctive genetic signatures at the single cell level (Greaves, 2010). On the other hand, the recent observations of a pre-metastatic gene signature in an early-stage primary breast tumor implying that metastatic cells may pre-exist in the primary tumor and are unlikely to be generated through natural selection via clonal evolution (Ramaswamy et al., 2003; van 't Veer et al., 2002; van de Vijver et al., 2002).

Despite the differences, the CSC model and clonal evolution model may both be operational during tumor initiation and progression. Specifically, tumor initiation may take place at the single-cell level by following either clonal evolution or the CSC model. Normal SCs, progenitor cells, and even differentiated cells could receive the malignant hit to initiate a tumor. However, during tumor progression, presumably only the CSCs possess properties like self-renewal that can endow them the advantage to undergo a combination of differentiation and clonal selection. These can be perfectly explained by a recent study on colon cancer: clonally selected tumor clones exhibit enhanced tumorigenic ability and CSC markers expression, indicating that CSC properties may be the key factor to make the clone dominant to undergo serial selection (Odoux et al., 2008). Perhaps only these CSCs could survive during this selection process and accumulate further mutations. Furthermore, step-wise mutations and clonal selection may 
enhance or maintain self-renewal properties to create the dominant CSC subclone and form genetically diverse units of evolutionary selection during cancer progression (Adams and Strasser, 2008).

\section{The Origin of CSCs}

The origin of CSCs has implications in understanding tumor heterogeneity and tumor development. The cellular origin of tumors in human cancer research is a question that remains largely unresolved. The CSC hypothesis may provide a direct speculation on this question by assuming that CSCs derive from their normal SC counterparts. There are several lines to support this point. Firstly, most reported CSCs appear to share similar markers to these on the corresponding normal SCs, implying that they might descend from same lineage. For instance, $\mathrm{CD}_{4} 4^{+}$subpopulation from breast cancers express many SC markers that are commonly expressed in the CD44 ${ }^{+}$normal SCs (Al-Hajj et al., 2003). Secondly, self-renewal is the most important trait of CSCs. Normal SCs already posses this capability and it is reasonable to think that SCs bestow this property to CSCs during transformation. Theoretically, it will be more difficult for the non-self-renewing differentiated cells to acquire this trait through multiple rounds of mutations that were reviewed in (Lawson and Witte, 2007; Li et al., 2007b). Thirdly, the recent two reviews ( $\mathrm{Li}$ et al., 2007b; Shackleton et al., 2009) that they demonstrate the longevity of SCs may allow greater opportunities to accumulate genetic changes in them. This thinking would be consistent with the idea that tumor transformation is not a single-hit but a multi-hit process. In contrast, more differentiated cells without self-renewal ability have a shorter life span such that they are likely to die or undergo terminal differentiation before enough 
mutations accumulate for full malignant transformation. Finally, evidence from both human primary cancer and animal tumor models supports the idea that tumor is a disease of SC origin. The Philadelphia chromosome is a hallmark for human chronic myelogenous leukemia (CML) (Fialkow et al., 1977), it is also found to be present in normal hematopoietic stem cell (HSC) (Miyamoto et al., 2000; Passegue et al., 2003), thus suggesting strongly that HSC are the targets of malignant transformation.

Recent studies in mouse tumor models demonstrate that cancer may also arise from committed progenitors (Haeno et al., 2009; Jordan et al., 2006). For example, over-expression of mixedlineage leukemia (MLL)- AF9 oncogene successfully induce leukemia from committed myeloid progenitors (Krivtsov et al., 2006). Since SCs are thought generally rare and quiescent, they may have less of a chance to acquire key mutations compared with the more enriched and faster dividing progenitors. All of these discussions support the idea that progenitor cells could also be targets of transformation during cancer evolution.

Another possible origin of CSCs might be cell fusion between SCs and other types of cells including other SCs, progenitor cells, mature differentiated cells, stromal cells and inflammatory cells. The cell fusion hypothesis may nicely fit the CSC hypothesis in that the combination of SCs with self-renewal ability with differentiated cells that have accumulated mutations could allow the fusion cells to attain fully neoplastic transformation (Wang, 2010). One example is that a renal cell carcinoma arises and metastasizes after allogeneic stem cell (HSC) transplantation. Tumor cells derived from the metastases exhibited a hybrid feature between both the host and donor cells (June, 2007). Other studies have shown that bone marrow derived cells (BMDCs) can fuse with neoplastic epithelium to promote tumor development and metastasis and that BMDCs 
-tumor cell fusions could be the source of CSCs (Rizvi et al., 2006). Recent studies on induced pluripotent SCs ( iPS) reveal that reprogramming differentiated human cells could initiate cancer development (Werbowetski-Ogilvie and Bhatia, 2008).

Taken together, SCs and progenitor cells are possible targets for tumorigenic transformation although cell fusion might provide another cellular origin of cancer development. Since tumor microenvironments play a critical role in tumor initiation and metastasis (Hu and Polyak, 2008a), it is possible that the microenvironment also influences the origin of cancer, as illustrated by the BMDCs and microphages-tumor cell fusions (Lu and Kang, 2009; Pawelek and Chakraborty, 2008). Identification of the normal cell populations targeted for tumorigenic transformation may facilitate the development of more effective cancer preventives.

\section{Normal Prostate Stem/progenitor Cells and Prostate CSCs}

Two fundamental traits of SCs, i.e., self-renewal and multi-potency, were first established in studies of hemopoiesis ( $\mathrm{Li}$ et al., 2007b; Weissman, 2000). The adult SCs are primitive cells with the self-renewal ability to differentiate into all different lineages of progeny and even to reconstitute an organ or regenerate the damage tissues, the best example of which are HSCs that can reconstitute the whole blood and rescue a lethally irradiated mouse (Tang et al., 2007; Weissman, 2000). Identification of various SCs has generated great hopes for cell therapies of neurodegenerative diseases, burn, diabetes, and heart diseases (Tu et al., 2002). On the other hand, although the HSCs can be defined as long-term (LT) HSCs, short-term (ST) HSCs, and multi-potent progenitor (MPP) cells, we still lack in-depth knowledge on the lineage development in most other solid tissue SCs including the prostate (Tang et al., 2007). 
Human prostate is a tubular-alveolar exocrine gland comprised of three distinct cell types (basal, luminal, and neuroendocrine $[\mathrm{NE}]$ cells) embedded in a fibro-muscular stroma. Basal cells form the layer along the basement membrane and luminal cells sit above the basal-cell layer and secret prostatic proteins into the lumen whereas NE cells are a minor population of neuronlike cells that produce biogenic amines and neuropeptides that support epithelial growth (AbateShen and Shen, 2000; Lawson and Witte, 2007; Tang et al., 2007). Luminal cells express prostate-specific antigen (PSA), prostatic acid phosphatase (PAP), androgen receptor (AR), and cytokeratins (CK) 8 and 18. In contrast, basal cells express CK5 and CK14 but not PSA and PAP (Abate-Shen and Shen, 2000; Lawson and Witte, 2007). However, some basal cells also express low levels of AR and BCL-2 and more than $80 \%$ of the proliferative cells reside in the basal layer. Unlike the majority of secretary luminal cells, NE cells are quiescent and do not express AR or PSA but express NE-specific markers such as chromogranin A and synaptophysin (Palapattu et al., 2009).

Evidence that strongly supports the existence of SCs in the prostate is from John Isaac's classic androgen cycling experiments (English et al., 1987). The observation that the adult rodent prostate can undergo multiple rounds of castration-induced regression and testosterone-induced regeneration indicates that a small population of SCs possesses the ability of to both self-renew and differentiate while the bulk, androgen-dependent, terminally differentiated cells lack such an ability (English et al., 1987). Several candidate populations of human prostate stem/progenitor cells have been reported including those expressing high levels of CD44, integrin a2 $\beta 1$, or CD133 (Tang et al., 2007). CD44 is expressed on most basal cells and has been implicated in tumor metastasis (Patrawala et al., 2007; Patrawala et al., 2006). The CD133 ${ }^{+}$cells are enriched 
in $\mathrm{CD} 44+/ \mathrm{a} 2 \mathrm{~B} 1^{\mathrm{hi}}$ basal cell population and possess high proliferation potential in vitro and the ability to produce prostate glandular structures (Richardson et al., 2004). Interestingly, two recent independent studies in the mouse prostate have identified two different populations of SCs. One, marked by CD117 (c-Kit), is localized in the basal layer (Leong et al., 2008) and the other, called castration-resistant Nkx3.1-expressing cells, in the luminal layer (Wang et al., 2009).

Identification and characterization of normal prostate SCs clearly have relevance to understanding the cell of origin for human PCa. The experimental evidence that most of the cells that survive castration appear to be basal rather than luminal cells has led to the traditional hypothesis that the basal-cell layer harbors self-renewing SCs (Abate-Shen and Shen, 2000; English et al., 1987). There are several pieces of evidence that support this hypothesis. First, some key molecules that normally regulate SC self-renewal and survival, e.g., p63, hTERT, and Bcl-2, are preferentially localized in the basal layer (Liu et al., 1997; Tang et al., 2007). Second, the proximal region of mouse prostatic ducts that highly expresses basal cell marker CK14 but not luminal marker CK8 is found to be the prostatic SC niche (Tsujimura et al., 2002). Furthermore, basal cells that express SC markers such as CD44, CD49f, CD117, Bc12, Tert, and p63 are all located in proximal region but not distal and intermediate regions.(Lawson and Witte, 2007; Leong et al., 2008) Third, when the basal-cell marker p63 is knocked out, mice are born without prostate or mammary gland (Mills et al., 2002). Forth, basal cells have been shown to differentiate into luminal cells (Collins et al., 2001; Lawson and Witte, 2007; Liu et al., 1997). Fifth, all primary normal human prostate (NHP) epithelial cells that can expand in vitro express basal-cell markers such as CD44, a2ß1, CK5, hTERT, and p63 but not luminal markers(Bhatia 
et al., 2008a), suggesting that these primary cells may contain a small number of prostate SCs. Finally, a single, basally localized $\mathrm{Lin}^{-} \mathrm{Sca}-1^{+} \mathrm{CD} 133^{+} \mathrm{CD} 44^{+} \mathrm{CD} 117^{+}$cell can generate prostatic glands when recombined with urogenital sinus mesenchyme and transplanted under the kidney capsule, strongly arguing for the presence of SCs in the basal layer. This defined SC population also possesses long-term self-renewal and can produce secretory prostatic product (Leong et al., 2008). Moreover, the SC marker CD117 is specifically expressed in the proximal region and enriched upon castration (Leong et al., 2008).

In human PCa, the majority of tumor cells express luminal markers such as PSA and AR and most prostate tumors have a notable lack of cells expressing basal-cell markers, in fact, loss of basal cell markers has been used in assisting the clinical diagnosis of PCa(Millikan and Logothetis, 1997; Oesterling et al., 1993). In the mouse prostate, the long-term BrdU labelretaining cells (or LRCs), which have been shown to possess functional SC properties in many other tissues, are localized in not only basal but also the luminal layer in the proximal region (Tsujimura et al., 2002), suggesting that the luminal cell layer may also harbor SCs. The luminal origin of prostatic SCs is further supported by recent demonstration of a rare population of luminal cells that expresses the regulator of prostate epithelial differentiation, the homeobox gene Nkx3.1, and manifest SC properties upon castration (Wang et al., 2009). These luminally localized SCs, termed CARNs for castration-resistant Nkx3.1-expressing cells, can self-renew and regenerate prostatic outgrowth with single-cell transplantations (Wang et al., 2009).

Intriguingly, Leong et al demonstrated that the synaptophysin-expressing NE cells are also enriched in $\mathrm{Lin}^{-} \mathrm{Sca}-1^{+} \mathrm{CD} 133^{+} \mathrm{CD} 44^{+} \mathrm{CD} 117^{+}$mouse prostate SCs (Leong et al., 2008). Huang et al studied the relationship between the SC marker CD44 and NE cells and found that most NE 
cells expressed CD44 (Palapattu et al., 2009). These latter observations raise the possibility that even NE cells might have some SC properties. More discussions on prostate CSCs will be presented in Chapter 2.

\section{CSCs and Metastasis}

If CSCs are the only cells that have the capacity to generate a tumor, theoretically they must be responsible for metastasis and therefore CSC-targeted therapies should be able to eliminate metastasis, the main killer of cancer patients. In other words, the CSC hypothesis will be considered incomplete without making connection to metastasis (Hermann et al., 2008; Hurt and Farrar, 2008; Li et al., 2007b; Wicha, 2006). In fact, many pieces of evidence support CSC involvement in metastasis. First, eight out of nine breast cancer specimens in which the first solid-tumor CSCs were reported were actually metastases (Al-Hajj et al., 2003; Steeg, 2006), implying that metastases may be enriched in CSCs. The CD44 ${ }^{+}$prostate CSC-enriched cells are also highly metastatic (Patrawala et al., 2006). Second, gene expression profile studies on PCa identified an 11-gene signature that included 'stemness' genes such as BMI-1 in highly metastatic PCa and also predicted poor outcomes in PCa patients (Glinsky, 2005; Glinsky et al., 2005). Also, the poorly differentiated aggressive human tumors are found to possess an ES celllike gene expression profile (Ben-Porath et al., 2008). In addition, early-disseminated breast cancer cells detected in the bone marrow are enriched in $\mathrm{CD} 44^{+} \mathrm{CD} 24^{-/ 10} \mathrm{CSC}$ phenotype (Balic et al., 2006). Third, in principle, only CSCs that are endowed with the self-renewal ability could founder a colony in a distant site while differentiated cells that generally lack the self-renewal capacity would not proliferate well to establish a metastatic colony (Fidler and Talmadge, 1986; 
Tu et al., 2002). Forth, EMT (epithelial-mesenchymal transition) plays an important role in metastasis and a recent study demonstrates that breast cancer cells induced to undergo EMT also acquires CSCs phenotypes (Mani et al., 2008). Fifth, tumor dormancy has long been recognized as a cause of metastasis in the clinic, particularly in breast and prostate tumors and metastasis can occur many years after treatment (Fidler and Talmadge, 1986; Li et al., 2007b). CSCs may stay quiescent and switch from being dormant to proliferative due to environmental changes, giving rise to recurrence and metastasis. Finally, development of resistance to therapeutics frequently signals the presence of metastatic lesions. Recent studies indicate that CSCs appear to be generally more resistant to chemical and radiation therapies (Barnhart and Simon, 2007). These discussions implies that CSCs could be the lethal seeds in Paget's "seed and soli hypothesis" to spread metastasis.

On the other hand, as is always the case, the real picture may be more complicated. For instance, the $\mathrm{CD} 44^{+} / \mathrm{CD} 24^{-}$breast CSCs do not seem to possess higher metastatic potential than the CD44/CD24 ${ }^{+}$cells when intra-cardiacally injected into mice although the two populations show differences by in vitro invasion assays(Sheridan et al., 2006). In addition, the molecule CD24 is, in fact, highly expressed in breast cancer metastases (Shipitsin et al., 2007). CD133 is another widely used CSC marker; however, Hermman et al reported that the CD133 ${ }^{+}$cell population alone could not produce metastasis in an orthotopic pancreatic cancer model but the $\mathrm{CD}_{133}{ }^{+} \mathrm{CXCR}^{+}$subpopulation showed strong metastasis (Hermann et al., 2007). Also, the $\mathrm{CD}_{133^{-}}$colon cancer cells have been shown to be even more aggressive and metastatic than their $\mathrm{CD}_{133^{+}}$counterparts although both populations could initiate tumor development (Shmelkov et al., 2008). These findings suggest that metastasis and tumor initiation might be processes 
mediated by distinct cancer cell populations (Hermann et al., 2008) and there might exist metastatic CSCs (Mimeault and Batra, 2007; Weigelt et al., 2005). The genetically-based clonal evolution hypothesis proposes that metastasis results from accumulation of genetic mutations and only the rare tumor cell clones that have attained the right and sufficient numbers of mutations will be "naturally selected" and able to disseminate (Campbell and Polyak, 2007; Polyak, 2007). This hypothesis is challenged by demonstrations that metastatic cells pre-exist in early-stage primary tumors (Singh et al., 2002; van 't Veer et al., 2002; van de Vijver et al., 2002). As a matter of fact, CSCs might actually be the 'naturally selected' clones in the clonal evolution model (Scheel et al., 2007). A putative model was proposed by using breast CSCs as an example (Weigelt et al., 2005)(Figure 1.4). In this model, oncogenic mutations occurring in a breast SC can cause the transformation to a breast CSC in a CSC niche, and the breast CSCs may change to metastatic CSCs to direct metastasis, and finally enter the distant organs to develop metastasis. These distant or tissue specific metastatic CSCs also need to interact with microenvironment. CSC may differentiate into a cancer progenitor cell with low ability to metastasize under a progenitor cell induced microenvironment. On the other hand, normal progenitor cells also could be the targets of transformation events, the outcome of which is that breast cancer progenitor cells will be produced, which may only initiate tumor development with less or limited potential to metastasize. The key point is that metastasis might be a critical feature for CSC, and microenvironment may play important roles in deciding the fate of CSCs. 


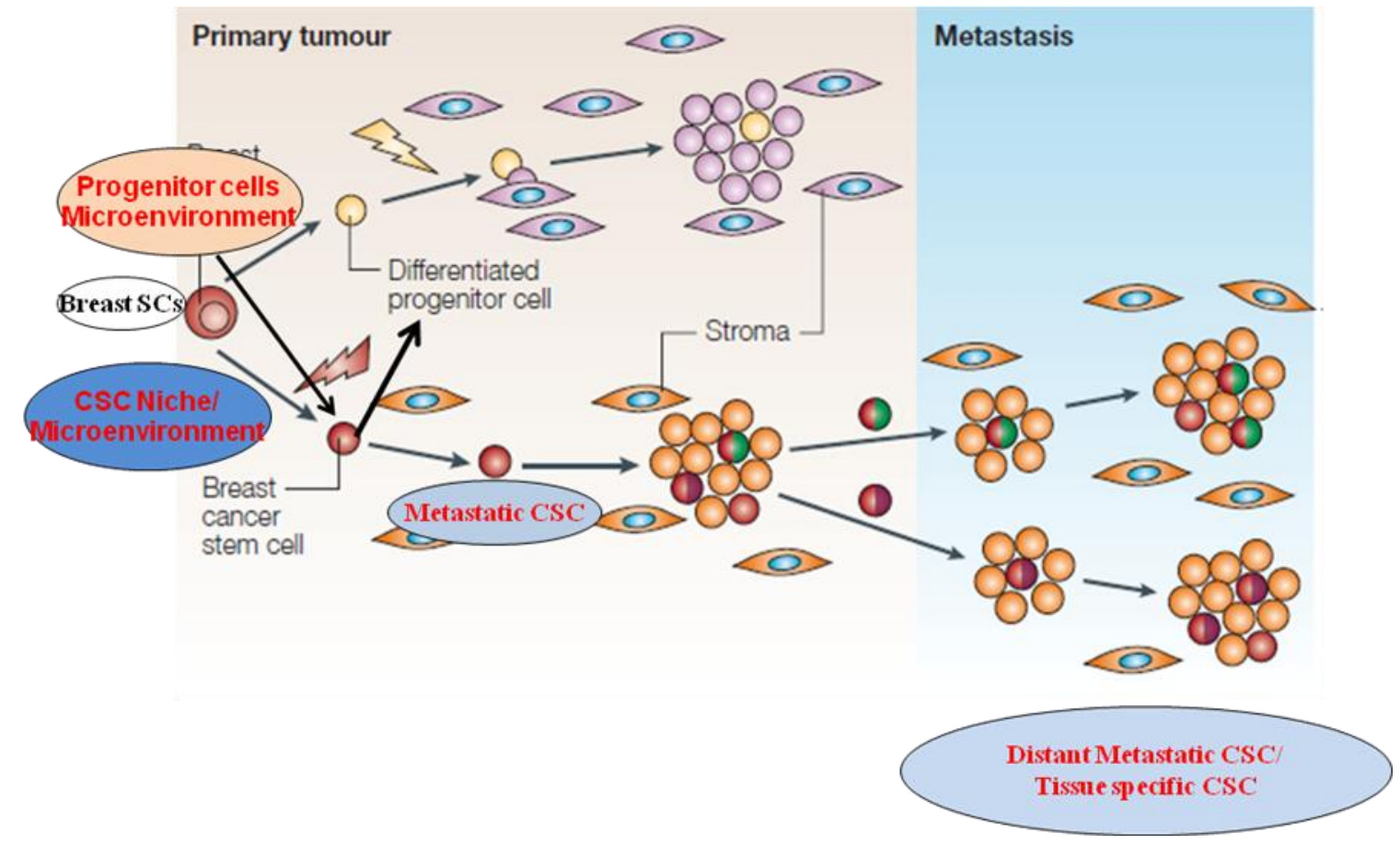

\section{Figure 1.4. A modified integrative model of breast cancer metastasis}

Oncogenic mutations occurring in a breast stem cell (red) can cause the transformation to a breast CSC in a CSC niche. The breast CSCs may change to metastatic CSC to direct metastasis, and finally enter the distant organs as a distant or tissue specific metastatic CSCs. The CSC may differentiate into a cancer progenitor cell with low ability to metastasize under a progenitor cell induced microenvironment. On the other hand, normal progenitor cells also could be the target of transformation events, the outcome of which is that breast cancer progenitor cells will be produced which may only initiate tumor development with less or limited potential to metastasize. The key point is that metastasis might be a critical feature of CSCs, meanwhile, microenvironment may play important role in deciding the fate of CSC (adapted from Weigelt B, et al., Nat Rev Cancer. 2005) 
In summary, the true interrelationship between CSCs and metastasis awaits more in-depth studies and it is anticipated that novel therapeutics that specifically target CSCs may also root out metastasis-seeding cells. 


\section{Chapter II: Assays for identification of Prostate Cancer Stem Cells*}

The CSC theory posits that only a small population of tumor cells within the tumor has the ability to reinitiate tumor development and is responsible for tumor homeostasis and progression. By this definition, the intrinsic CSC properties must be tested and exhibited by tumor growth ability in a foreign microenvironment. In human CSC studies, growing a human tumor in immune deficient mouse is very complicated and influenced by multiple factors. To a certain degree, the methods used to analyze CSC properties are just as same important as the intrinsic CSC properties. Some debates against CSC hypothesis are actually related to method. Herein, we present a comprehensive review of the experimental strategies that our lab has been using in assaying potential PCa SCs in culture, xenograft tumors, and primary tumor samples.

In the past 7 years, putative CSCs, or tumor initiating cells, have been reported for many human solid tumors (Table 2.1), including brain tumors (Singh et al., 2004), melanoma (Schatton et al., 2008), and cancers of the breast (Al-Hajj et al., 2003; Ginestier et al., 2007), colon (Dalerba et al., 2007; O'Brien et al., 2007; Ricci-Vitiani et al., 2007; Todaro et al., 2007), pancreas (Hermann et al., 2007; Li et al., 2007a), liver (Yang et al., 2008), lung (Eramo et al., 2008), and head and neck (Prince et al., 2007). Unlike CSC studies in other cancers, the direct demonstration of tumor-initiating cells from primary human prostate tumors has not been reported. Most CSC studies in prostate cancer mainly use cancer cell lines or xenograft models (Tang et al., 2007). Several important principles have emerged from these studies.

First, most CSCs have been identified using cell surface markers for the corresponding normal tissue stem/progenitor cells, suggesting that normal and cancer SCs share some phenotypic markers. Second, interestingly, CD44 and CD133 have been used to identify many

* This chaptor is adapted from Li, et al., Methods Mol Biol. 2009, with permission of copyright 
Table 2.1. CSC Studies in Human Solid Tumor (2003-2008)

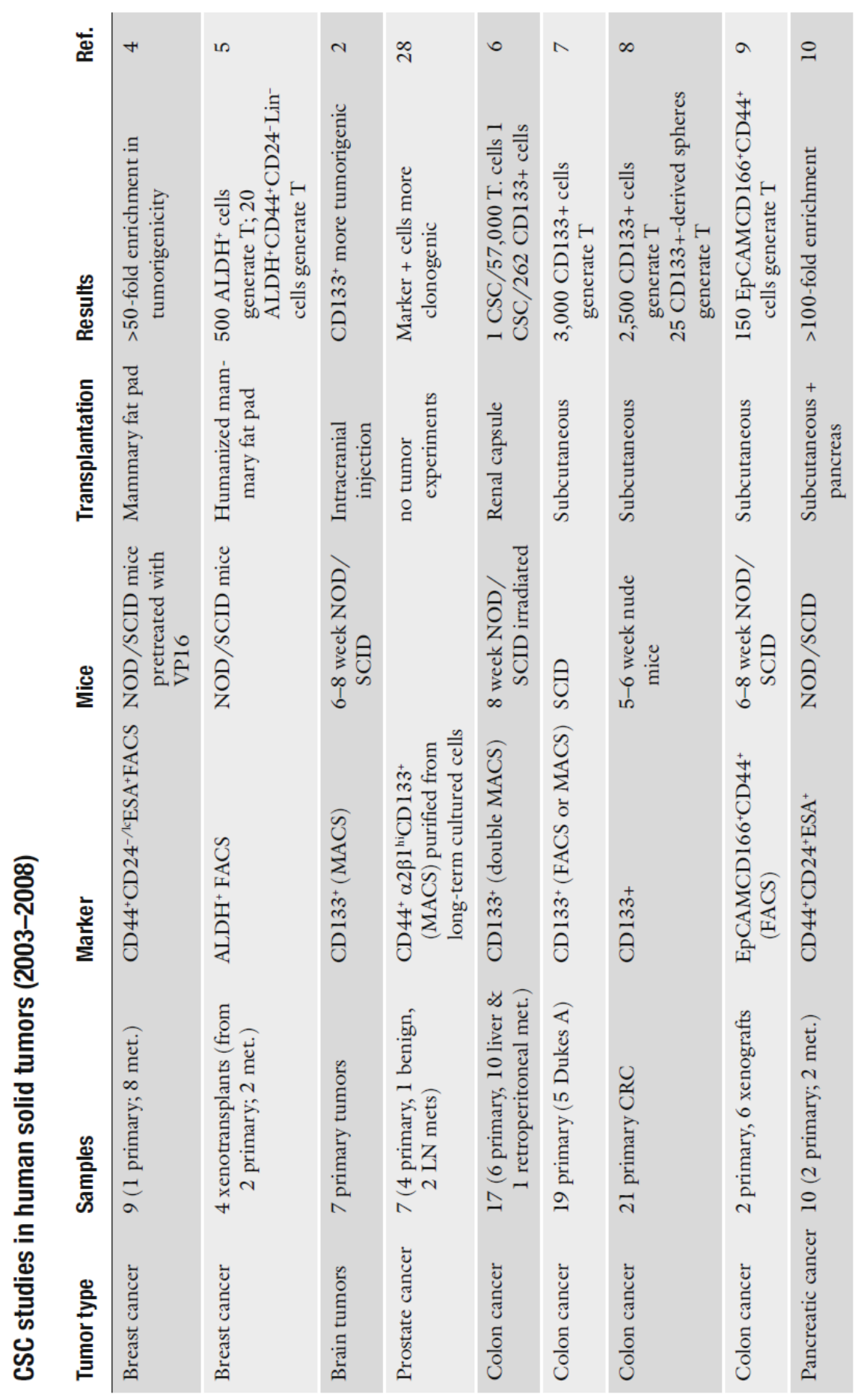


Table 2.1. CSC Studies in Human Solid Tumor (2003-2008)

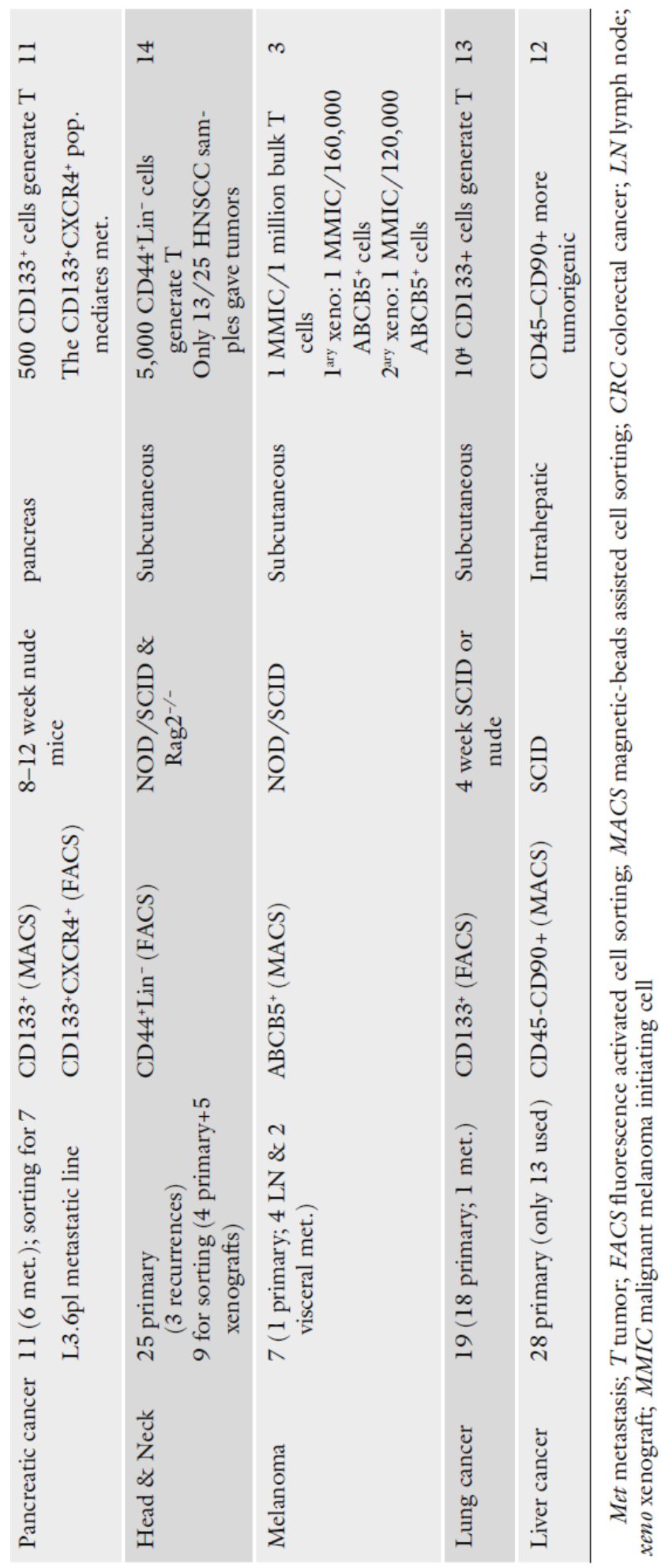


types of CSCs. For example, CD44 has been used to enrich for breast, colon, pancreatic, liver, and head and neck CSCs whereas CD133 for CSCs in lung and colon cancers and glioma (Table 2.1). Some other markers may be tumor specific, e.g., breast CSCs have a CD $44^{+} / \mathrm{CD}_{2} 4^{-}$ phenotype (Al-Hajj et al., 2003) whereas pancreatic CSCs possess the CD44 ${ }^{+} / \mathrm{CD} 24^{+}$phenotype (Li et al., 2007a). Third, in a particular tumor, CD44 and CD133 may identify distinct and/or overlapping populations of tumor stem/progenitor cells. For instance, both CD133 (O'Brien et al., 2007; Ricci-Vitiani et al., 2007; Todaro et al., 2007) and CD44 (Dalerba et al., 2007) have been utilized as the positive selection marker for colon CSCs. The same two markers have also been employed to independently select for pancreatic CSCs (Hermann et al., 2007; Li et al., 2007a). In both cases, the interrelationship (inclusive, exclusive, or hierarchical) between the CD133 and CD44 selected CSCs remains unclear. These observations emphasize the important point that the CSC population is likely heterogeneous, as elucidated in LSCs (Hope et al., 2004), and also raise the possibility that combining CD44 and CD133 may enrich for more primitive CSCs. Fourth , CSCs are only operationally or functionally defined. Perhaps one of the most important criteria is that putative CSCs possess an enhanced ability to initiate serially transplantable tumors that phenotypically recapitulate patient tumor histology (Clarke et al., 2006; Tang et al., 2007). In all of the above mentioned CSC studies (Table 2.1), "naked" tumor cells were injected into the immunodeficient mice, implying that putative CSCs possess an intrinsic ability to establish a "niche" in a foreign microenvironment. Fifth, on the other hand, reconstitution of CSC activity and tumordevelopment of human tumor cells in mice represents an extremely challenging task (Hill, 2006; Tang et al., 2007) involving numerous variables 
Table 2.2. Variables affecting the outcome of human prostate cancer tissue/cell grafting and establishment in mice

\begin{tabular}{|l|l|}
\hline Recipient mice & Strains (see Tables 3) \\
& Preconditioning (pre-injection of etoposide; \\
& irradiation; injection of anti-NK antibodies, \\
& "humanizing" implantation sites, etc.)
\end{tabular}


Table 2.3-1. Mouse strains commonly used in CSCs

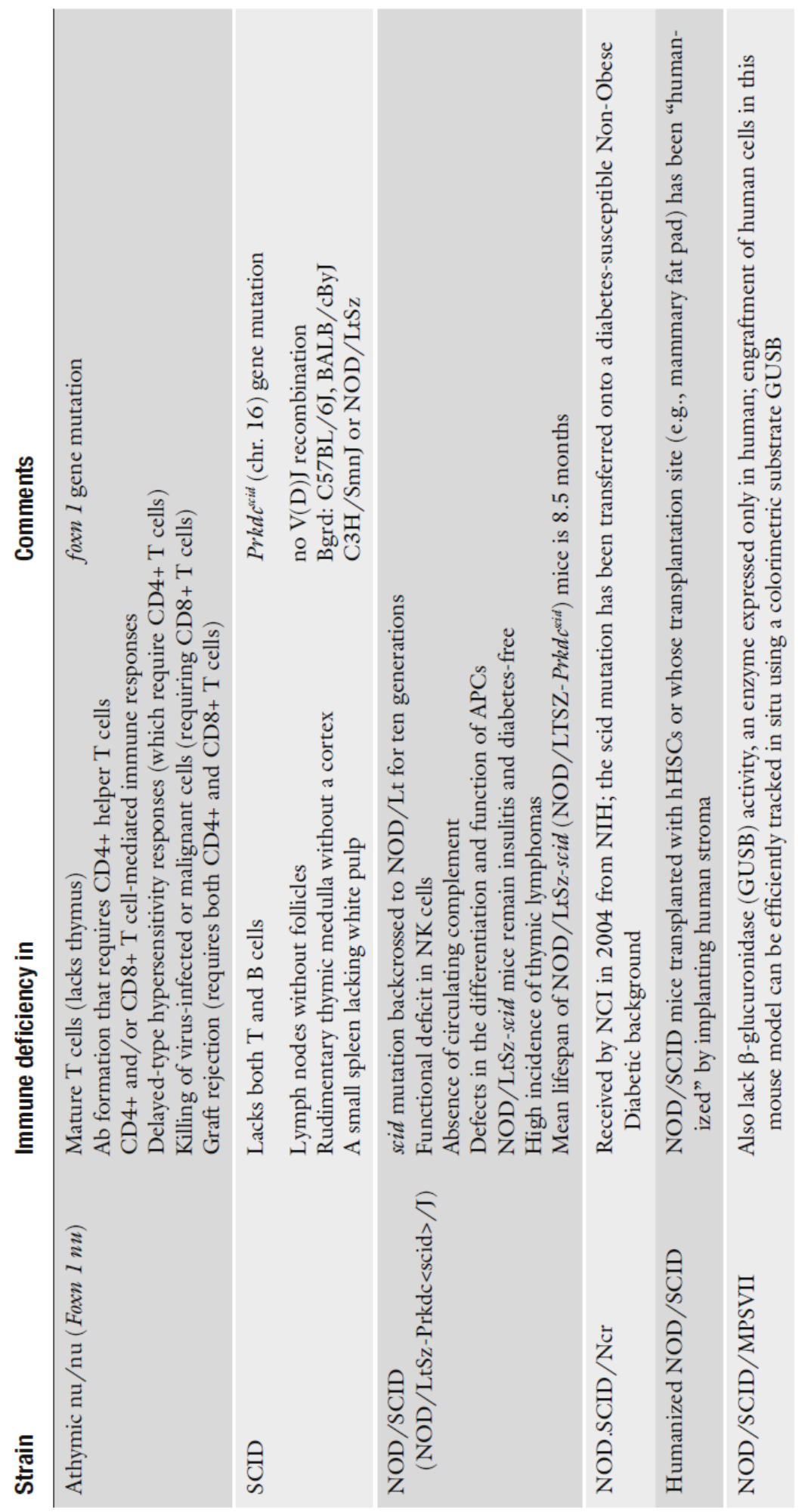


Table 2.3-2. Mouse strains commonly used in CSC studies

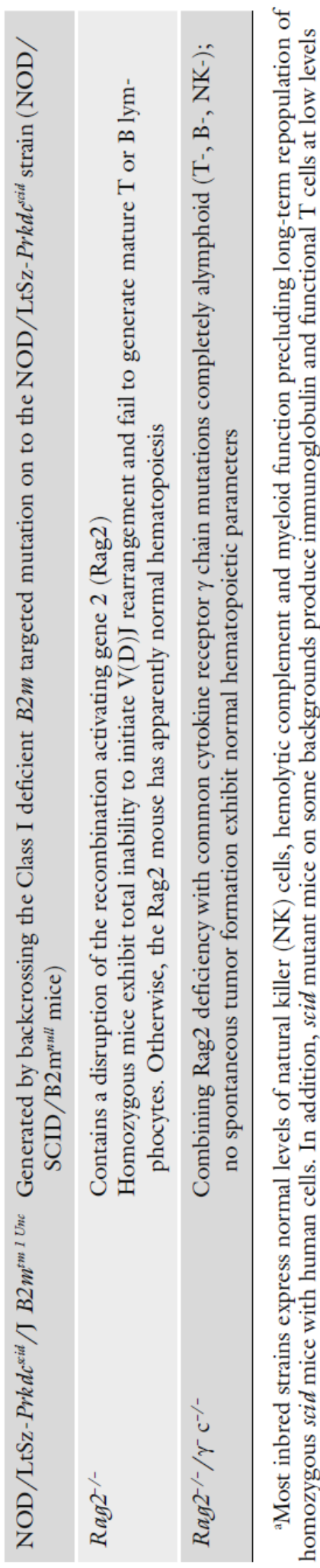


associated with both tumors (availability, heterogeneity, stage/grade, size, quality, digestion/purification/implantation methods, etc.; Table 2.2 ) and recipient mice (strains, degree of immune deficiency, preconditioning, injection/implantation sites, etc.; Table 2.3). Consequently, different studies have a wide variety of "empirical" details that cannot be interpreted readily and reconciled scientifically. For instance, although some tumorigenic subsets were implanted "orthotopically," many others were injected at ectopic sites, in particular, subcutaneously (s.c.) or under the kidney capsule (KC) (Table 2.1). Sixth, as predicted, CSCs seem to be more resistant to antitumor therapeutics, including chemotherapy and radiation (Hermann et al., 2007; Wang, 2007; Yu et al., 2007). Of clinical significance, the abundance of CSCs significantly increases in breast cancer patients who have received prior therapies (Yu et al., 2007).

Most CSC studies in prostate cancer mainly use cancer cell lines or animal models (Tang et al., 2007). Pioneering studies from Drs. Collins/Maitland provided evidence that putative human prostate epithelial SCs bear the $\mathrm{CD} 44^{+} /$a $2 \beta 1^{+} / \mathrm{CD} 133^{+}$phenotype (Collins et al., 2001; Richardson et al., 2004). In 2005, Collins et al reported that prostate tumor cells with the same surface phenotype represent potential prostate CSCs, although tumor experiments were not reported in this study (Collins et al., 2005). Using several xenograft prostate tumors (Du145, LAPC4, and LAPC9), we have shown that the $\mathrm{CD} 44^{+}$cell population is enriched in prostate CSCs and that PCa cells are organized as a tumorigenic hierarchy (Li et al., 2008; Patrawala et al., 2007; Patrawala et al., 2006; Patrawala et al., 2005)(Fig. 2.1). First, putative CSCs that can initiate serially passageable spheres and serially transplantable tumors are marked by CD44 
Stem/progenitor cells(minor subsets in the tumor)

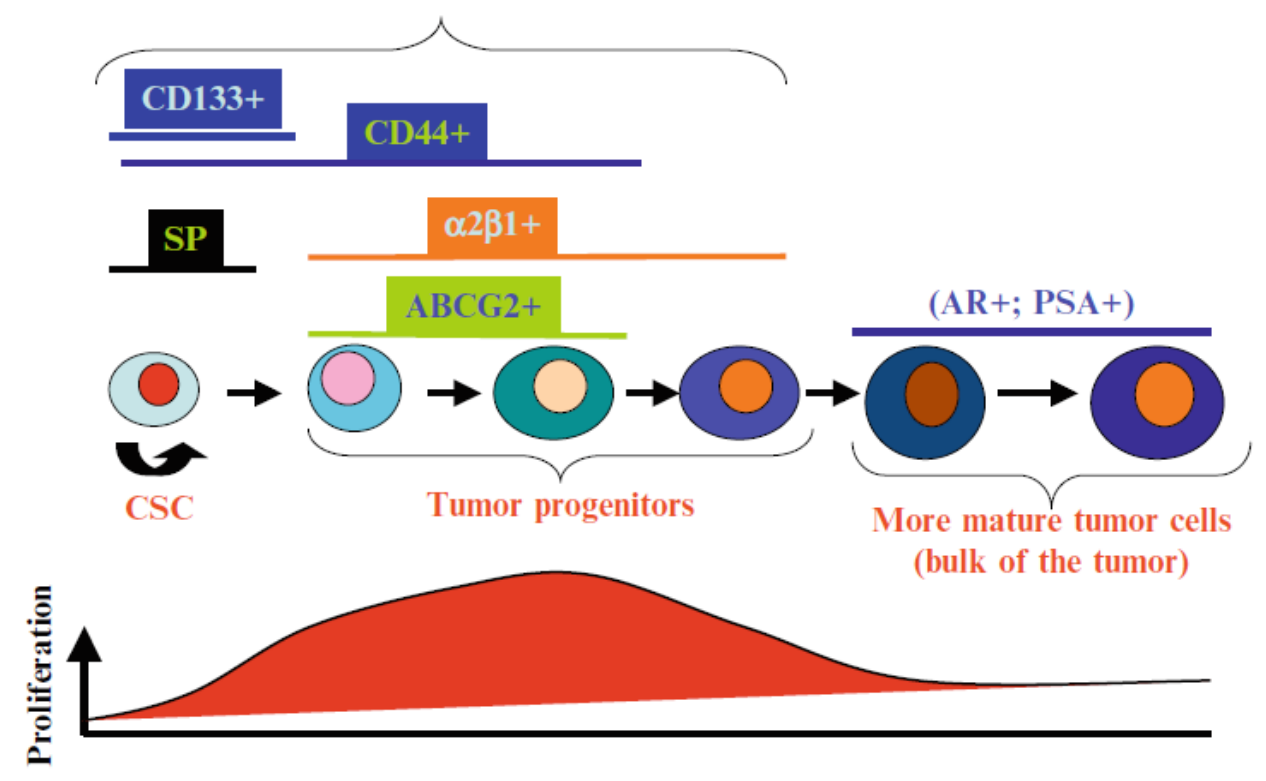

Fig. 2.1. A model of hierarchical organization of tumorigenic PCa cells

(Taken from Li et al., Methods Mol Biol. 2009 with permission) 
expression and constitute the minority. Most of the $\mathrm{CD} 44^{+}$cells in the spheres or tumors are not proliferating. Importantly, essentially all metastatic activity resides in the $\mathrm{CD}_{4} 4^{+}$cell population (Patrawala et al., 2006). Second, the side population (SP) also contains tumorigenic cells and 97\% of the SP cells are CD44 ${ }^{+}$. Third, in contrast to the SP and CD $44^{+}$cells, the $a 2 \beta 1^{+}$and $\mathrm{ABCG}_{2}{ }^{+} \mathrm{PCa}$ cells identify fast proliferating tumor progenitors. Fourth, essentially all $\mathrm{ABCG} 2^{+}$ and $>80 \%$ a $2 \beta 1^{+}$cells are encompassed in the $\mathrm{CD} 44^{+}$population. Therefore, the CD44 $/ a 2 \beta$ $1^{+}$cell population is highly enriched in tumor-initiating cells, whereas the $\mathrm{CD} 44^{-} / \mathrm{a} 2 \beta 1^{+}$cells virtually lack tumorigenicity. Fifth, most $\mathrm{CD} 44^{+} \mathrm{PCa}$ cells are $\mathrm{AR}^{-}$and can give rise to $\mathrm{AR}^{+}$cells in the spheres and tumors, thus indicating their ability to self-renew and undergo asymmetric division. Recent work by others has confirmed the presence of stem-like PCa cells in cell lines (Gu et al., 2007; Miki et al., 2007).

Practically, CSC studies involve (a) sample preparation, (b) candidate cell purification, and (c) in vitro and (d) in vivo analysis and characterizations of CSC properties. In the following we present some basic in vitro and in vivo assays applied to prostate CSC studies. Sample preparation, candidate cell purification and related protocols can also be found in our publications (Jeter et al., 2009; Li et al., 2008; Patrawala et al., 2007; Patrawala et al., 2006; Patrawala et al., 2005)and on our website (http://sciencepark.mdanderson.org/tanglab/protocols).

In order to reconstitute a tumor in immune-deficient mice, hundreds or thousands of cultured or xenograft tumor cells must be injected. This phenomenon suggests that perhaps only a small population of cells in the bulk tumor or the entire culture holds the ability to reinitiate a tumor. Therefore, for a candidate CSC population, once it is isolated from the bulk tumor by 
removing the stromal cells, it must be purified by either SC markers or non-marker based purification strategies. Then it can be tested for its enriched tumor associated properties by in vitro assays as well as in vivo tumor experiments (Tang et al., 2007). Though there are still a lot of questions in this field, I briefly discuss some commonly used assays to purify CSCs and characterize the functions of CSCs.

SC markers are among the most widely used to study CSCs. By using flow cytometry or MACS microbeads based system, distinct cancer cell populations can be isolated and applied in in vivo and in vitro analyses. For instance, CD44 has been regarded as the most successful CSC marker with which one can purify a CSC enriched population in PCa (Patrawala et al., 2006). One advantage of cell surface marker based analysis is to allow researcher to combine several markers to select the specific functional subpopulation inside the tumor and further distinguish the CSC population from progenitor population or more mature populations. For example, $\mathrm{CD} 44^{+} / \alpha 2 \beta 1^{+}$subpopulation exhibits much higher tumorigenic potential and could be the CSCs in $\mathrm{PCa}$, whereas the $\mathrm{CD} 4^{-} / \mathrm{a} 2 \beta 1^{+}$population may represent the progenitor population with less tumorigenic potential (Patrawala et al., 2007).

Side Population: Identifying the Side Population, or SP, is a flow cytometry-based technique using the exclusion dye hoechst 33342 and has been widely used as a marker of SC in both normal tissue and tumor (Brown et al., 2007; Patrawala et al., 2005). For example, 0.1\% SP cells were found in LAPC9 tumors and these SP cells demonstrated 100-1000-fold higher tumorigenicity than the corresponding non-SP cells. SP tumor cells also expressed high levels of 
Beckman-Coulter Altra
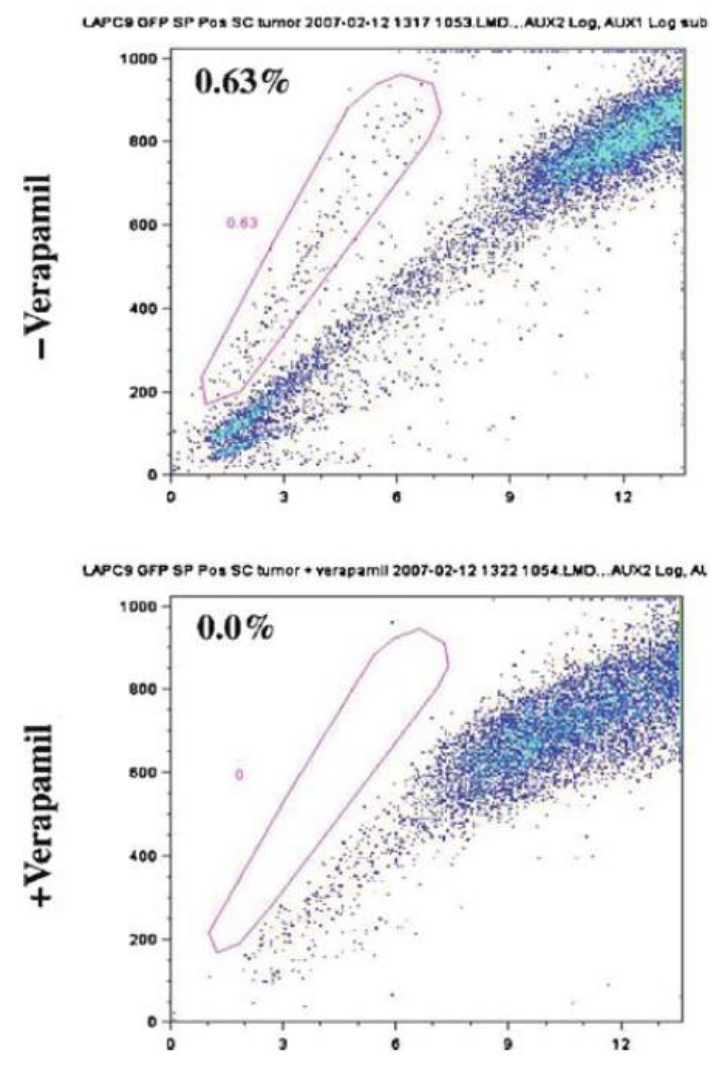

BD FACSAria
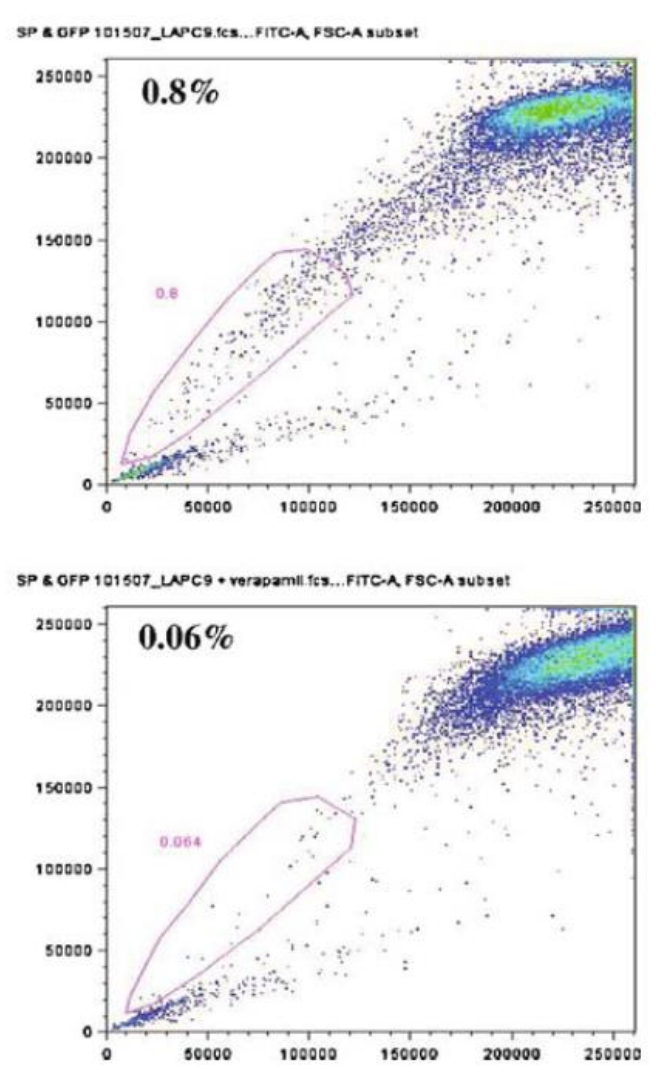

Fig. 2.2. SP analysis of LAPC9 tumor cells on two different flow cytometers 
"stemness" genes such as $\beta$-catenin and BMI-1(Patrawala et al., 2005). A recent study found that the SP was detected in both benign and malignant prostate tissues that expressed basal cell markers (Brown et al., 2007). Whether these SP cells selected from primary human PCa samples also possess CSC properties and high tumor-initiating ability is currently under investigation. It is important to note that different flow cytometers often give different SP profiles (Fig.2.2). For example, when samples are run on a Beckman-Coulter, the SP displays as distinct population on the side. However, when the same cells are analyzed on a BD Biosciences FACSAria SORP, the SP displays as a tail of the MP (Fig. 2.2).

Clonogenic or Sphere Formation Assays can be performed by putting cancer cells (including cultured or primary cancer cells) into a semi-solidified medium (such as soft agar or Matrigel) or plating onto low attachment plates such that they cannot adhere to the substrate or to each other, so that single-cell derived colonies or spheres will form. The clonogenic or sphere formation assay provides an important way to assay CSC properties in vitro. Sufficient evidence supports that SCs can maintain its spectrum of differentiating abilities in a three-dimensional sphere structure (Lawson and Witte, 2007; Tang et al., 2007). Recently, sphere formation assays were utilized in central nervous system (CNS) tumors and melanomas to demonstrate that the tumor cells in a sphere also hold the ability to re-initiate tumor in mice (Singh et al., 2003). In PCa, xenograft tumor LAPC4 was able to form clongenic spheres and these sphere-forming cells, although rare, were enriched during serial passage and developed tumors in NOD/SCID mice (Tang et al., 2007).

Clonal morphology analysis can be done by plating cells at very low numbers in culture. Cultured cancer cells or primary cells could form three morphologically distinct clones: 
holoclones, maraclones and paraclones. Holoclones were shown to have enriched CSC properties, as they contain the tumor-initiating subset cells in prostate cancer cell line (Li et al., 2008). The analysis recently is applied in not only in CSCs analysis, but also in some anti-CSC drug screening (Klonisch et al., 2008).

BrdU pulse-labeling strategy can be adapted to identify slow-cycling cells. The principle is as follows. After a long period of BrdU “pulse," i.e., from days to weeks, most or all cells with proliferative capacity will be labeled (i.e., will incorporate the thymidine analog $\mathrm{BrdU}$ into the DNA). Then the samples will be washed free of BrdU. Following an extended period of culture or maintenance in the absence of BrdU (i.e., the "chase"), samples will be terminated and used to stain for BrdU. Progenitors which cycle faster (i.e., have a shorter cell-cycle time) will gradually dilute out BrdU and eventually become BrdU-negative whereas the putative SCs, which are generally more quiescent, will stay BrdU-positive and be identified as the label-retaining cells or LRCs. The LRCs in the bulge of mouse epidermis and the proximal tubules of mouse prostate have been shown to possess SC properties (Shen et al., 2008; Tsujimura et al., 2002). The LRCs in human breast tumors coexpress mammary epithelial SC markers and seem to have certain SC properties (Clarke et al., 2003). Human PCa cell spheres and xenograft tumors (Patrawala et al., 2006) and nasopharyngeal carcinomas also possess slow-cycling LRCs (Zhang et al., 2007).

The "gold" standard of measuring CSC properties is that the candidate CSC population should possess enhanced capacity, in an experimental system, to initiate tumors that recapitulate patient tumor histology and can be serially transplanted (Table 2.2) (Clarke et al., 2006; Tang et al., 2007). Therefore, tumor experiments must be done to show this critical capacity associated with the presumed CSCs. Nevertheless, "reconstituting" human tumor development in mice, 
whether from primary tumor pieces or from dissociated cells, is extremely complicated involving numerous variables associated with both (donor) tumors and (recipient) mice (Tables 2.2, 2.3). Several major lessons on reconstituting human PCa development in NOD/SCID mice have been learnt. The first thing that we need to pay attention to is the clarification about tumor take vs. tumor growth. These two terms are often used interchangeably, by mistake. Tumor take refers simply to "grafting," i.e., the initially implanted human tumor pieces (or cells) have stayed or grafted in the mouse but there is no apparent tumor growth. Tumor growth (or regeneration or development) refers to obviously enlarged tumor burden compared to the initial implants. Any confusion of these two concepts may cause some false judgment. As we mentioned earlier, we are not injecting "naked" tumor cells into mice and Matrigel is commonly used to co-inject with tumor cells since Matrigel could resemble the complex extracellular three-dimensional matrix found in many tissues (Kleinman and Martin, 2005). In the most commonly used route of implantation, i.e., s.c., we have noticed that with cultured PCa cells, increasing Matrigel concentrations from $25 \%$ to $50 \%$ dramatically improves tumor take rate as well as tumor growth (unpublished observations). Furthermore, we need pay great attention on tumor implantation sites. Based on transplant sites, there are roughly two xenograft models: the orthotopic model in which injected/implanted human tumor cells/ pieces are implanted into the mouse prostate (dorsal prostate, or DP; anterior prostate, or AP) and the ectopic model in which human PCa cells/pieces are injected/implanted outside the mouse prostate, including s.c. and kidney capsules (KC). Among these sites, the s.c. site is the most sensitive and consistent site in reconstituting 
A Tumor development of SP and CD44 LAPC9-GFP cells implanted at different sites

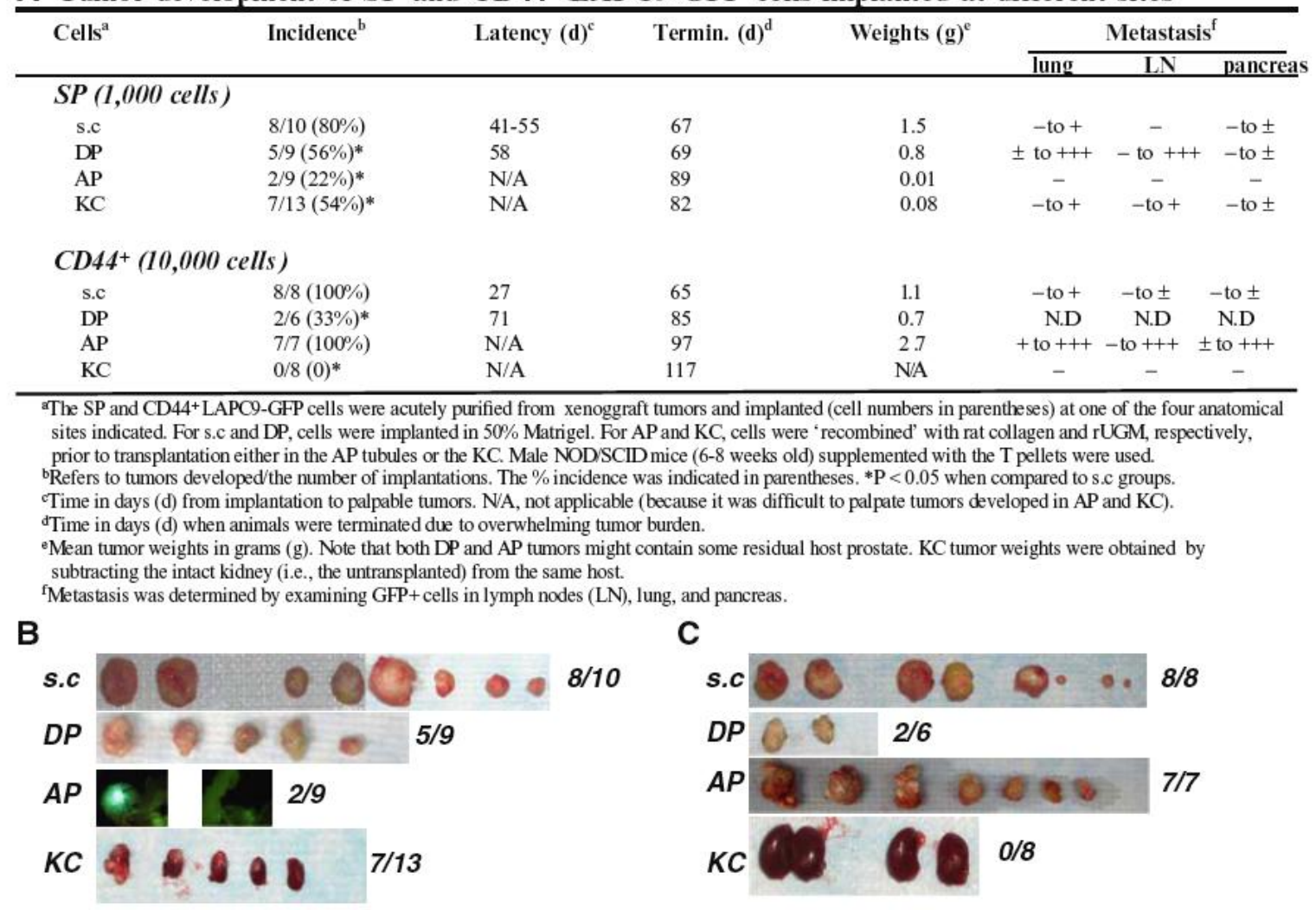

Fig. 2.3. Comparison of tumor development of prostate CSC populations (i.e., the SP and $\mathrm{CD44}^{+}$cells acutely purified from the GFP-tagged LAPC9 tumors) implanted at different sites

(A) Table presentation of results. (B) and (C) Tumor images of SP (B) and CD44 ${ }^{+}(\mathrm{C})$ LAPC9 cells implanted at different sites. In B, the two AP tumors were very small and therefore the GFP images were shown. In $\mathrm{C}$, none of the eight transplants in four kidneys developed into tumors SP analysis of LAPC9 tumor cells on two different flow cytometers. 
tumor development of prostate CSC-enriched cells in NOD/SCID mice. CSC-enriched PCa cell populations, including both SP (Patrawala et al., 2005) and CD44 (Patrawala et al., 2006) cells, when implanted into the 4 different sites, i.e., s.c., DP, AP, and KC, significant differences were observed with respect to tumor development (Fig.2.3). With both SP and CD44 ${ }^{+}$cells purified from LAPC9-GFP cells, the s.c.-implanted cells demonstrated the highest tumor regeneration (Fig.2.3). Differences between the SP vs. CD $44^{+}$cell populations were also observed in terms of tumor regeneration (Fig. 2.3). Additionally, the s.c. site is also the most sensitive and consistent site in reconstituting tumor development of primary HPCa pieces or cells in NOD/SCID mice. In fact, when tumor pieces or single cells from primary HPCa samples (6 Gleason 6 tumors, 8 Gleason 7 tumors, and 4 Gleason 8/9 tumors) were similarly implanted into the s.c., KC, or AP sites, the subcutaneum was also found to be the most sensitive site for tumor regeneration (Fig.2.4). However, s.c. is not the best model for metastasis. Consistent with previous study by using bulk HPCa cells in mouse DP injection (Kitadai et al., 1995), the prostate-implanted PCa cells show more extensive metastasis. In the experiments carried out with the SP and CD44 LAPC9-GFP cells, when we examined the $\mathrm{GFP}^{+}$tumor cells that disseminated into the lymph nodes (LN), lung, and pancreas, we observed that cells injected into DP (for SP cells) or AP (for CD $44^{+}$cells) showed more extensive dissemination than the s.c.-implanted cells ( Fig. 2.3A ). These observations are consistent with the long-established concept that the orthotopically implanted tumor cells demonstrate higher metastatic capacity.

With the above-discussed CSC assays in mind, we have proposed the following criteria to functionally or operationally define the putative CSCs (Tang et al., 2007). First, the presumptive CSC must be prospectively purified. When purifying candidate populations of CSCs, lineage 


\section{A Gleason 7}
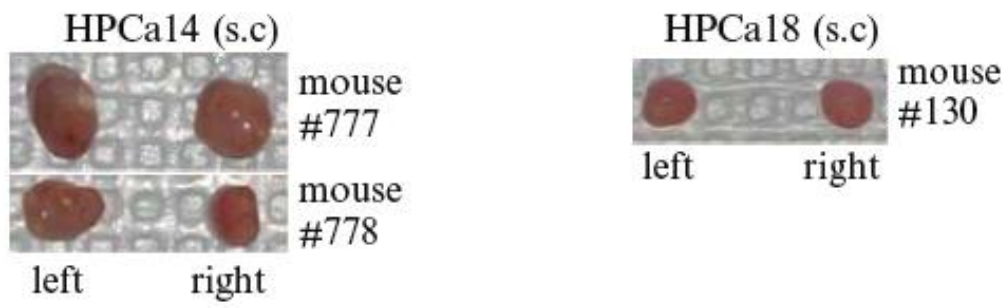

B Gleason 8
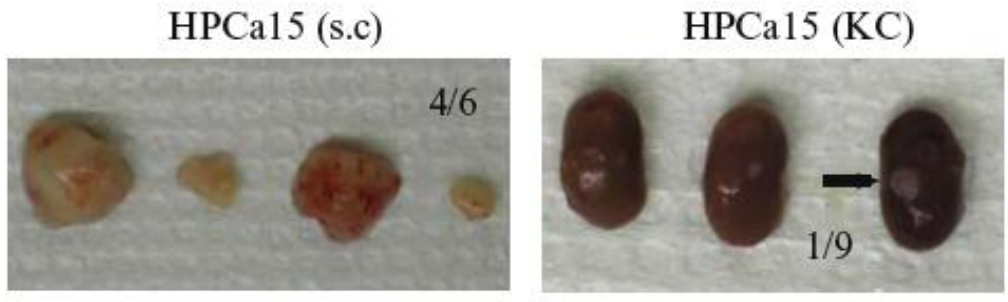

C Gleason 9
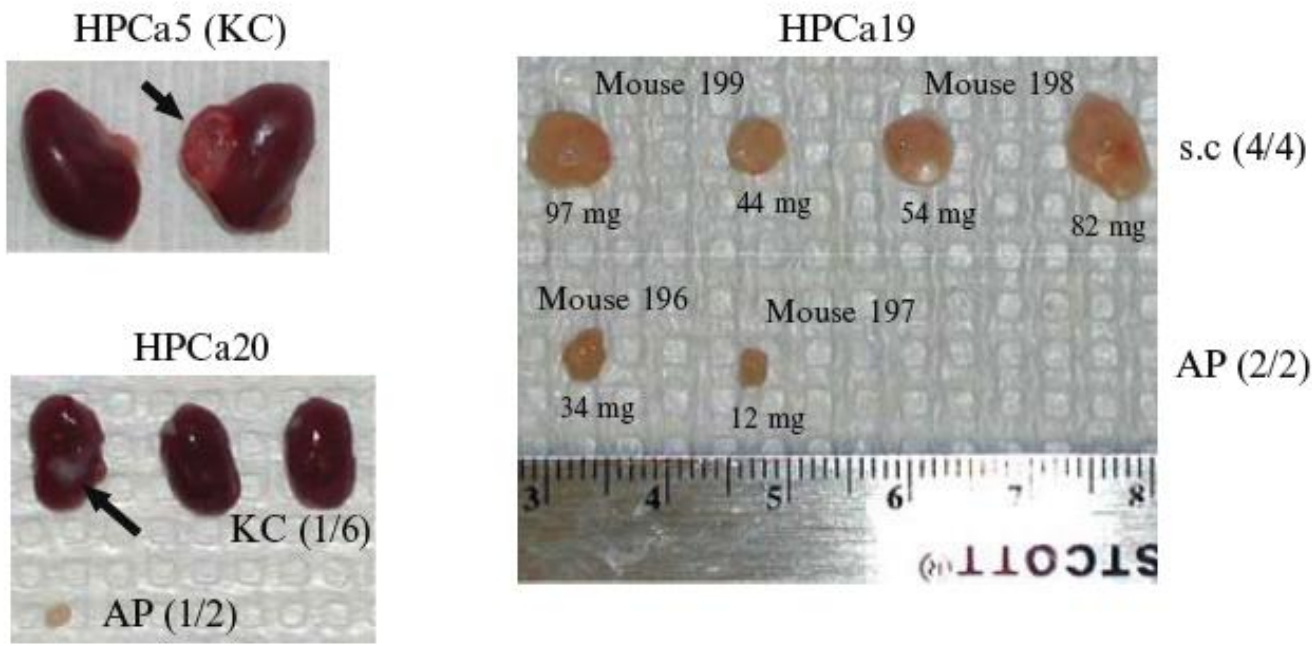

Fig. 2.4. The s.c. site is also the most sensitive microenvironment to reconstitute tumordevelopment from pieces or cells of the primary PCa samples

Shown are representative images of tumors regenerated from Gleason grade 7(A), 8 (B), and 9 (C) primary prostate tumor samples. Note obvious differences in the sizes of s.c. vs. AP or KC regenerated tumors. 
selection should be performed to remove "irrelevant" cells such as stromal and blood cells that may contain other SCs, including mesenchymal SCs and HSCs. Second, in vivo tumorigenicity experiments must be done to show that such cell populations, freshly purified and without extensive expansion in vitro, are enriched in tumor-reinitiating cells. When feasible, serial tumor xenotransplantation should be carried out to determine whether the tumors derived from the putative CSCs could be transplanted for multiple generations. Third, the reconstituted as well as serially xenotransplanted tumors should histologically resemble the original patient tumor. Fourth, importantly, the presumptive CSC population, or a subpopulation within, has to be studied to show that they possess certain intrinsic biological properties (extensive proliferative capacity, self-renewal, differentiation, etc.) normally associated with the SCs. Only when these conditions are fulfilled can one confidently claim that the candidatete population of tumor cells under investigation is enriched in potential CSCs or tumor-initiating cells. It is important to bear in mind that such tumorigenic populations are likely to be heterogeneous with true CSCs representing perhaps a very small fraction. 


\section{Chapter III: PC3 Human Prostate Carcinoma Cell Derived from Holoclones Contain Self-Renewing Tumor-Initiating Cells*}

\section{Introduction}

Every normal tissue or organ comprises multiple resident cell types that are heterogeneous with respect to their morphologies, functions, and gene and protein expression patterns. This cellular heterogeneity has been thought to reflect mostly the developmental and maturation stages of various normal stem and progenitor cells (Raff, 2003). Pioneering work by Barrandon and Green more than 20 years ago (Barrandon and Green, 1985) showed that when primary human keratinocytes were put in culture, their abilities to establish a clone were related to the heterogeneity in cell size —only cells $11 \mu \mathrm{m}$ in diameter could form a clone whereas cells $12 \mu \mathrm{m}$ were irreversibly committed to terminal differentiation. Their subsequent work (Barrandon and Green, 1987) in clonal cultures revealed three distinct types of clones with profoundly different proliferative capacity. The holoclone contains tightly packed small cells and has the greatest replicative capacity, and $<5 \%$ of the colonies formed by the cells of a holoclone abort and terminally differentiate. In contrast, the paraclone is a loosely packed clone of large cells with a short replicative life span —after $<15$ cell generations, paraclones uniformly abort and terminally differentiate. The third type of clone, the meroclone, contains a mixture of cells of different proliferative potential and is a transitional stage between the holoclone and the paraclone (Barrandon and Green, 1987). Recent work reveals that keratinocyte holoclones contain selfrenewing stem cells and that the ability to form a holoclone is an intrinsic property of the adult stem cells of the hair follicle (Claudinot et al., 2005).

\footnotetext{
* This chaptor is adapted from Li et al, Cancer Res. 2008, 68, 1820-5 with permission of copvright
} 
Tumor development to a certain degree resembles and has been likened to a "caricature" of normal tissue histogenesis and organogenesis (Sell and Pierce, 1994). Indeed, most human tumors are heterogeneous in their cellular composition (Dexter et al., 1978; Heppner, 1984; Weiss, 2000). Although many posit that tumor cell heterogeneity is of a genetic basis associated with inherent high genomic instability in tumor cells, the heterogeneous cellular composition in tumors has also been hypothesized, early on, to be the consequence of abnormal tumor stem cell differentiation (Pierce, 1974). On the other hand, cancer cell lines seem to have the unlimited proliferation ability. What is the model of these non-stop growth trends is still unknown. Recent our lab's work revealed that $\mathrm{CD} 44^{+}$and $\mathrm{CD} 44^{+} / \alpha 2 \beta 1^{+}$represented CSC population respectively (Patrawala et al., 2007; Patrawala et al., 2006). These work along other's studies supported that cancer cell line may also contain CSC. Additionally, similar to primary keratinocytes, longterm-cultured squamous cell carcinoma (Locke et al., 2005)and many other epithelial cancer cells (Locke et al., 2005; Tang et al., 2007) can also form different types of clones in culture. Only a small percentage of cells are endowed with the ability to establish holoclones, whereas the majority forms paraclones and meroclones (Locke et al., 2005; Tang et al., 2007) (Figure 3.1). Like keratinocyte holoclones, cancer cell holoclones have been hypothesized to contain self-renewing stem-like cells although direct evidence supporting this hypothesis is still lacking. Additionally, CSC is very difficult to detect in some HPCa cell lines due to short of appropriated markers. For instance, almost 100\% PC3 cells expressed CD44 whereas SP can not be detected in PC3 cells due to the extremely low level (Patrawala et al., 2006; Patrawala et al., 2005). It is possible to apply clonal morphology as an effective way to distinguish CSC from bulk of cells in such cancer cell lines like PC3. Hence, in this study, we used PC3 cells, an androgen receptor- 
negative, undifferentiated prostate cancer cell line, as the model system to directly test this hypothesis.

\section{Materials and Methods}

Cells, animals, and reagents PC3 cells were obtained from the American Type Culture Collection and cultured in RPMI containing 7\% of heat-inactivated fetal bovine serum (FBS). Nonobese diabetic/severe combined immunodeficient (NOD/SCID) mice were initially purchased from The Jackson Laboratory, and the breeding colonies were established in our animal facility (Patrawala et al., 2007; Patrawala et al., 2006; Patrawala et al., 2005)and maintained in standard conditions according to the institutional guidelines. Most chemicals and reagents were from Sigma unless otherwise specified.

Establishment of PC3-GFP cells Basic retroviral and lentiviral procedures have been previously described (Bhatia et al., 2008b; Bhatia et al., 2005). Briefly, 293FT packaging cells were transfected with pLL3.7-GFP lentiviral vector, together with the packaging plasmids, using Fugene 6 . The virus-containing medium was collected 48 to $72 \mathrm{~h}$ later and passed through a $0.45-\mu \mathrm{m}$ filter to remove debris. PC3 cells were then infected with virus at a multiplicity of infection of 50 and essentially $100 \%$ cells were infected.

Single-cell cloning by limiting dilution PC3-GFP cells (70\% confluent) were harvested in trypsin $(0.25 \%)$ /EDTA and resuspended in fresh medium to generate a single-cell suspension with a density of 10 cells $/ \mathrm{mL}$. Then, $100 \mu \mathrm{L}$ single-cell suspension was dispensed into each well 
in a 96-well culture plate. After plating, each well was checked under both an Olympus BX50 fluorescence microscope and a phase-contrast microscope. Wells containing only a single cell were marked, and wells with no cells or with more than one cell were excluded. These single-cell wells were checked daily and maintained in RPMI-7\% FBS. When the colony grew confluent, they were transferred to six-well dishes. Clones in six-well dishes were maintained until nearly confluent and then some of them were frozen and some were replated into T-75 flask or used for tumor experiment.

Senescence-associated $\boldsymbol{\beta}$-galactosidase staining Basic procedures were previously described (Bhatia et al., 2005).

\section{Subcutaneous tumor cell implantation and purification of tumor cells from xenograft}

tumors Basic procedures were previously described (Li et al., 2009; Patrawala et al., 2007; Patrawala et al., 2006; Patrawala et al., 2005). For tumor development assays, cells derived from holoclones, meroclones, or paraclones were injected s.c. in 50\% Matrigel into the flanks of NOD/SCID mice. To purify tumor cells, PC3 xenograft tumors were aseptically dissected out from animals and minced into 1-mm3 pieces in RPMI-7\% FBS. After rinsing in the same medium (2x), tumor tissues were incubated with 1x Accumax (1,200-2,000 units/mL proteolytic activity containing collagenase and DNase; Innovative Cell Technologies, Inc.) at $20 \mathrm{~mL} / 1 \mathrm{~g}$ tissue for $30 \mathrm{~min}$ at room temperature under rotating conditions. A single-cell suspension was obtained by filtering the supernatant through a $40-\mu \mathrm{m}$ cell strainer, and the cell suspension was then gently loaded onto a layer of Histopaque-1077 gradient $\left(1 \times 10^{6}-3 \times 10^{6}\right.$ cells $/ \mathrm{mL}$ Histopaque 
in a total of 3-mL volume) and centrifuged at $400 \times \mathrm{g}$ for $30 \mathrm{~min}$ at room temperature. $\mathrm{RBC}$, dead cells, and debris were eliminated from the bottom of the tube and live nucleated cells were collected at the interface. Then, the single-cell suspension was used for tumorigenesis or serial transplantation experiments.

Immunophenotypic characterizations of PC3 cell clones Bulk-cultured PC3 or PC3-GFP cells, or clone-derived cultures were plated on glass cover slips and used in immunofluorescence staining for CD44, a $2 \beta 1$ integrin, and $\beta$-catenin using monoclonal antibodies and protocols described previously (Patrawala et al., 2007; Patrawala et al., 2006; Patrawala et al., 2005).

\section{Results and Discussion}

\section{Prostate Cancer Cells in Clonal Cultures Exhibit Distinct Clonal Morphologies}

Our laboratory has been studying normal human prostate epithelial and prostate cancer stem and progenitor cells (Bhatia et al., 2008b; Bhatia et al., 2005; Patrawala et al., 2007; Patrawala et al., 2006; Patrawala et al., 2005; Tang et al., 2007). Our recent work has shown the existence of stem-like cells in cultured as well as xenograft-derived prostate cancer cells (Patrawala et al., 2007; Patrawala et al., 2006; Patrawala et al., 2005; Tang et al., 2007). Cultured epithelial cancer cells, when plated at clonal densities, have been shown to behave like primary keratinocytes and form colonies of distinct morphologies (Locke et al., 2005; Tang et al., 2007). Indeed, when PC3

(Fig. 3.1.A) and Du145 (Fig. 3.6.B) prostate cancer cells were cultured at clonal densities, in 2 weeks clones resembling keratinocyte holoclones, meroclones, and paraclones could be easily 

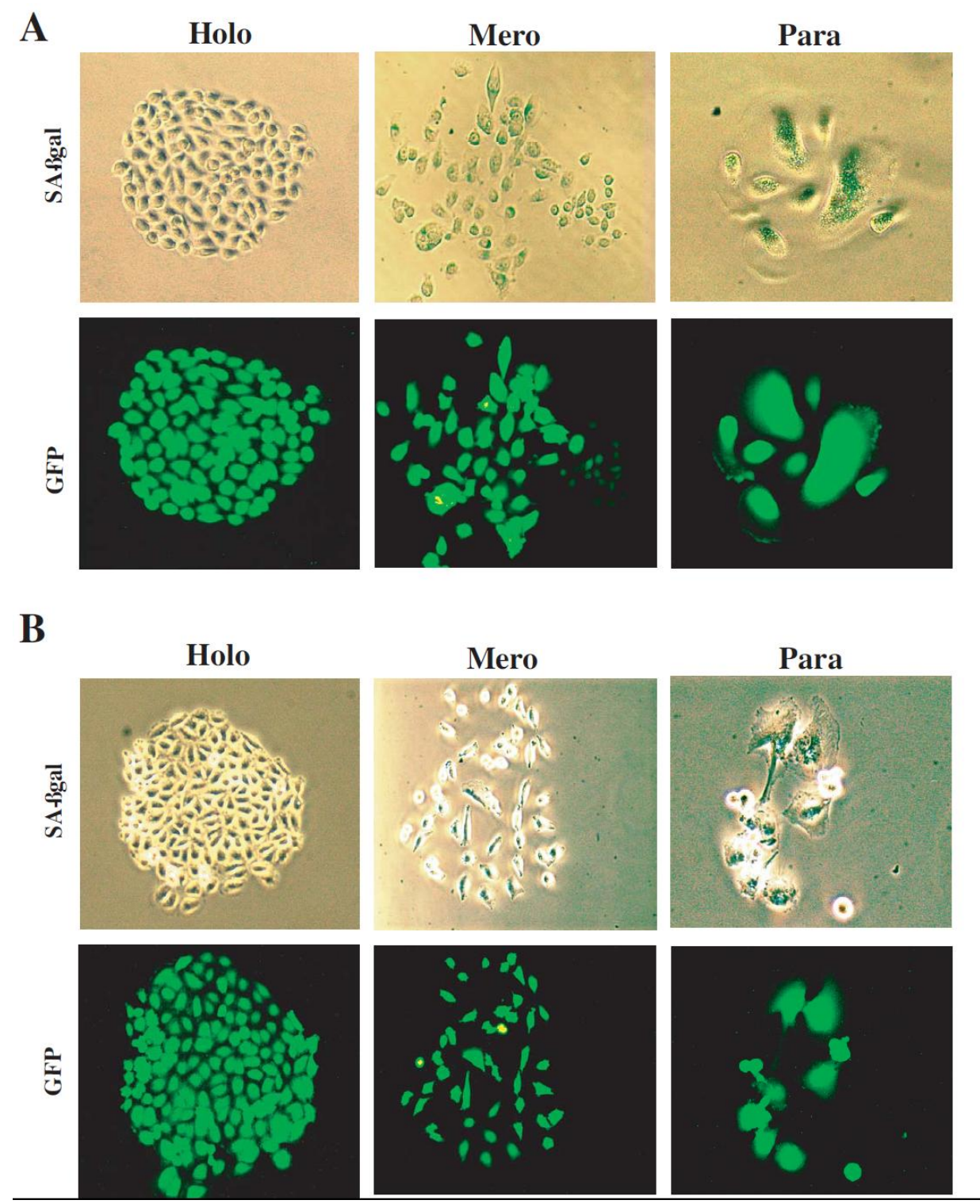

Figure 3.1. Cultured PCa cells exhibit three distinct clonal morphologies

PC3 (A) and Du145 (B) cells were cultured at clonal densities for 1 week. GFP images of holoclones (Holo), meroclone (Mero), or paraclones (Para) were shown at the bottom panels and phase-relief images of SA- $\beta$ gal staining (Bhatia et al., 2005) at the top. Original magnifications, $\mathrm{x} 100$. 
identified. Like keratinocyte clones (Barrandon and Green, 1985; Barrandon and Green, 1987; Claudinot et al., 2005), prostate cancer cell holoclones consisted of tightly packed, small cells and paraclones of larger and fewer cells, whereas the meroclones were made of cells of intermediate sizes and numbers (Fig. 3.1). Importantly, although cells in prostate cancer cell holoclones were homogenously small and senescence-associated $\beta$-galactosidase (SA- $\beta$ gal) negative, most cells in paraclones were flat and large and SA- $\beta$ gal positive (Fig. 3.1), suggesting that the latter cells were mostly senescent and non-proliferative. Many cells in meroclones were also SA- $\beta$ gal positive (Fig. 3.1.A). These results reveal that prostate cancer cells are heterogeneous in establishing different types of clones.

\section{Different PC3 Cell Clones Possess Distinct Proliferative and Self-renewing Capacities.}

We previously showed that Du145 cell holoclones could be serially passaged and sustain longterm expansion in culture (Tang et al., 2007). In this study, we focused on PC3 cells, which lack androgen receptor expression, are completely undifferentiated, highly tumorigenic and metastatic, and have been considered the most malignant prostate cancer cell line (Patrawala et al., 2007; Patrawala et al., 2006; Patrawala et al., 2005). We plated PC3-GFP cells at 1 cell per well in 96-well plates, and 2 hours after plating (to prevent cell division) we marked and selected those wells that contained only 1 viable cell (Fig. 3.2 A). Among the three 96-well plates studied, 72 wells satisfied our selection criteria (see Materials and Methods) and were followed up at the

clonal level (Fig. 3.2. B). In 2 weeks, 7 clones (10\%) developed into typical holoclones and 49 cells (68\%) formed paraclones, whereas 16 clones (22\%) were meroclones (Figs. 3.2 and 3.3). 


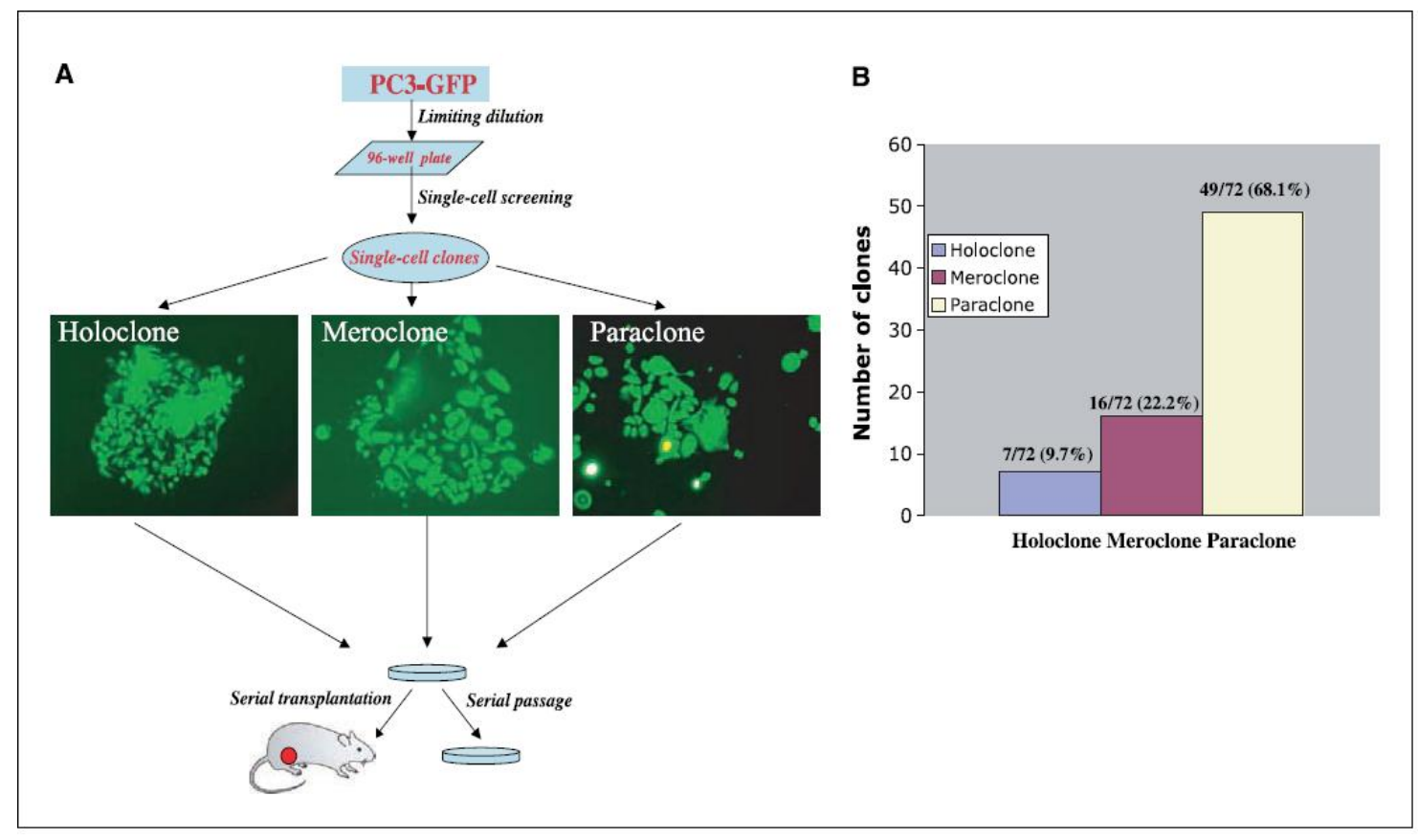

\section{Figure 3.2. Clonal Heterogeneity of PC3 Cell}

A, schematic depicting the procedure of deriving PC3 cell clonal cultures and functional assays. The GFP images are representative of three types of clones. Magnification, X100. B, quantification of each types of clones 2 wk after plating. 


\begin{tabular}{llr} 
A & \multicolumn{1}{c}{ Survival } & Aborted \\
\cline { 2 - 3 } $\begin{array}{l}\text { Clone } \\
\text { type }\end{array}$ & $6 / 7(85.7 \%)$ & $1 / 7(14.3 \%)$ \\
\hline Holoclone & $6 / 16(31.2 \%)$ \\
Meroclone & $11 / 16(68.8 \%)$ & $5 / 160)$ \\
Paraclone & $1 / 49(2.0 \%)$ & $48 / 49(98.0 \%)$ \\
\hline
\end{tabular}

B

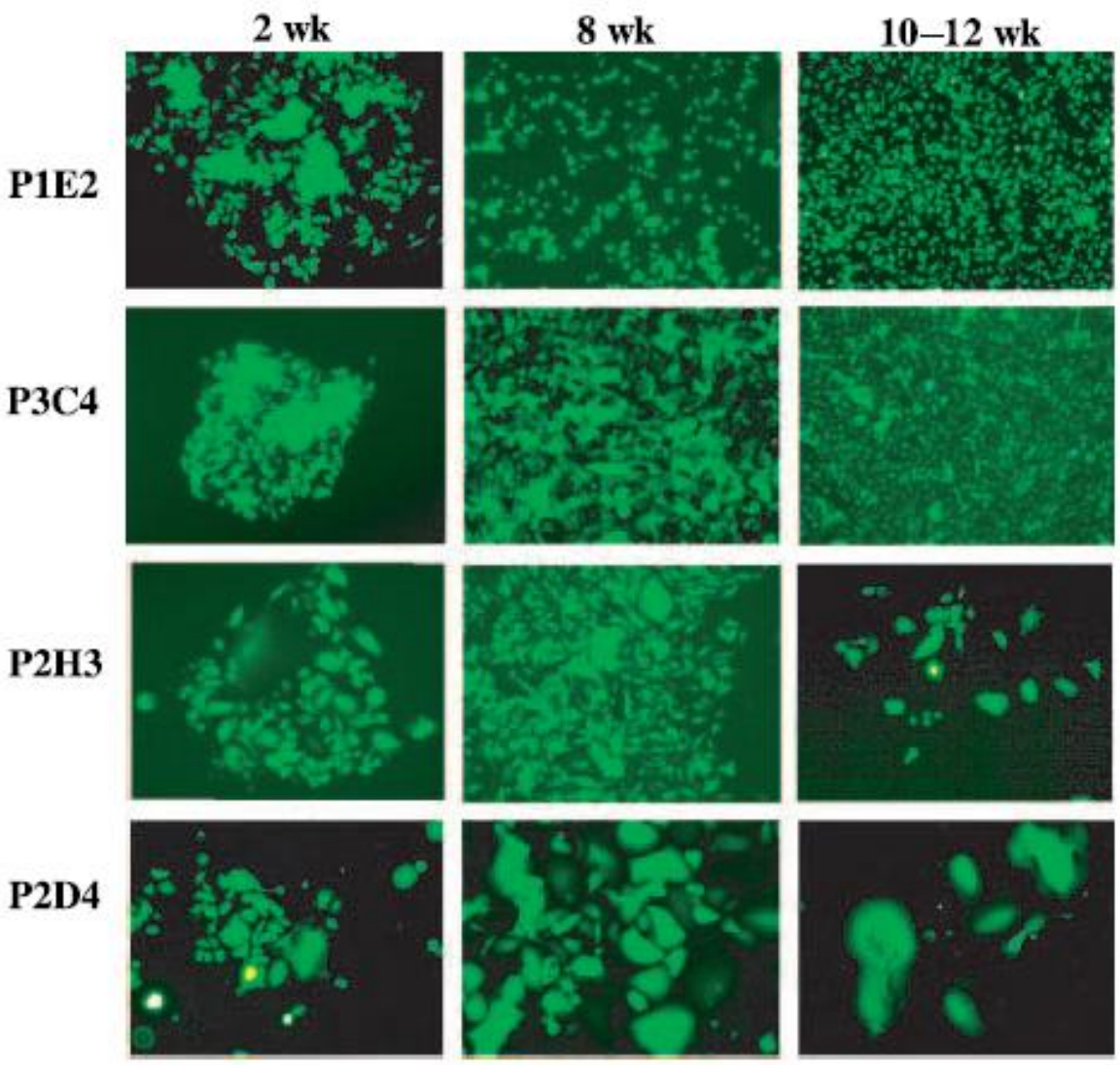

Figure 3.3 Developmental fate of three types of clones during the 12-wk follow-up period

A. Tble showing the "survival" and "abort" percentages of three types of clones 8 wk after initial plating. B. representative GFP images of two holoclones (P1E2 and P3C4), one meroclone $(\mathrm{P} 2 \mathrm{H} 3)$, and one paraclone (P2D4). Original magnifications, X200. 
We then followed the development of each individual clone in vitro through serial passaging and, for several clones, we also carried out serial tumor transplantation experiments (Fig. 3.2A; see below). As shown in Fig. 3.3B, the distinct morphologies of three types of PC3 clones were obvious at 2 weeks after single-cell plating. By 8 weeks, although most holoclones proliferated robustly and only one holoclone aborted, most (i.e., 98\%) of the paraclones, on the other hand, had aborted and thus been terminated (Fig. 3.3A and B). Meroclones showed intermediate behavior: By 8 weeks, 5 of the 16 clones (31\%) were abolished and could not be further propagated (Fig. 3.3. A). By 10 to 12 weeks after initial plating, 6 of the 7 holoclone-derived PC3 cells were still robustly proliferating whereas the remaining paraclone colony and the rest of the meroclones all aborted (Fig. 3.3.B, right panels). In all "aborted" clones, cells were generally big and flat (e.g., Fig. 3.3.B, bottom middle and right panels) and SA- $\beta$ gal positive (not shown). All six holoclone-derived PC3 cells could be continuously propagated for $>6$ months.

When the P1E2 holoclone-derived cells were re-plated at clonal densities, they were able to regenerate the full spectrum of clonal heterogeneities within 1 week (Fig. 3.4.A).

\section{PC3 Holoclones, But not Paraclones or Meroclones, Contain Stem-like Cancer Cells that Could Initiate Tumor Development and Sustain Serial Tumor Transplantation.}

The above serial cell passaging and re-plating experiments show that the PC3 holoclones contain self-renewing cancer cells or CSCs that can sustain long-term propagation in culture. The gold standard in measuring CSC activity is that the candidate cell populations must be able to initiate serially transplantable tumor development (Clarke et al., 2006; Tang et al., 2007). Therefore, we determined and compared the tumor-initiating capacities of PC3 holoclones, meroclones, and 

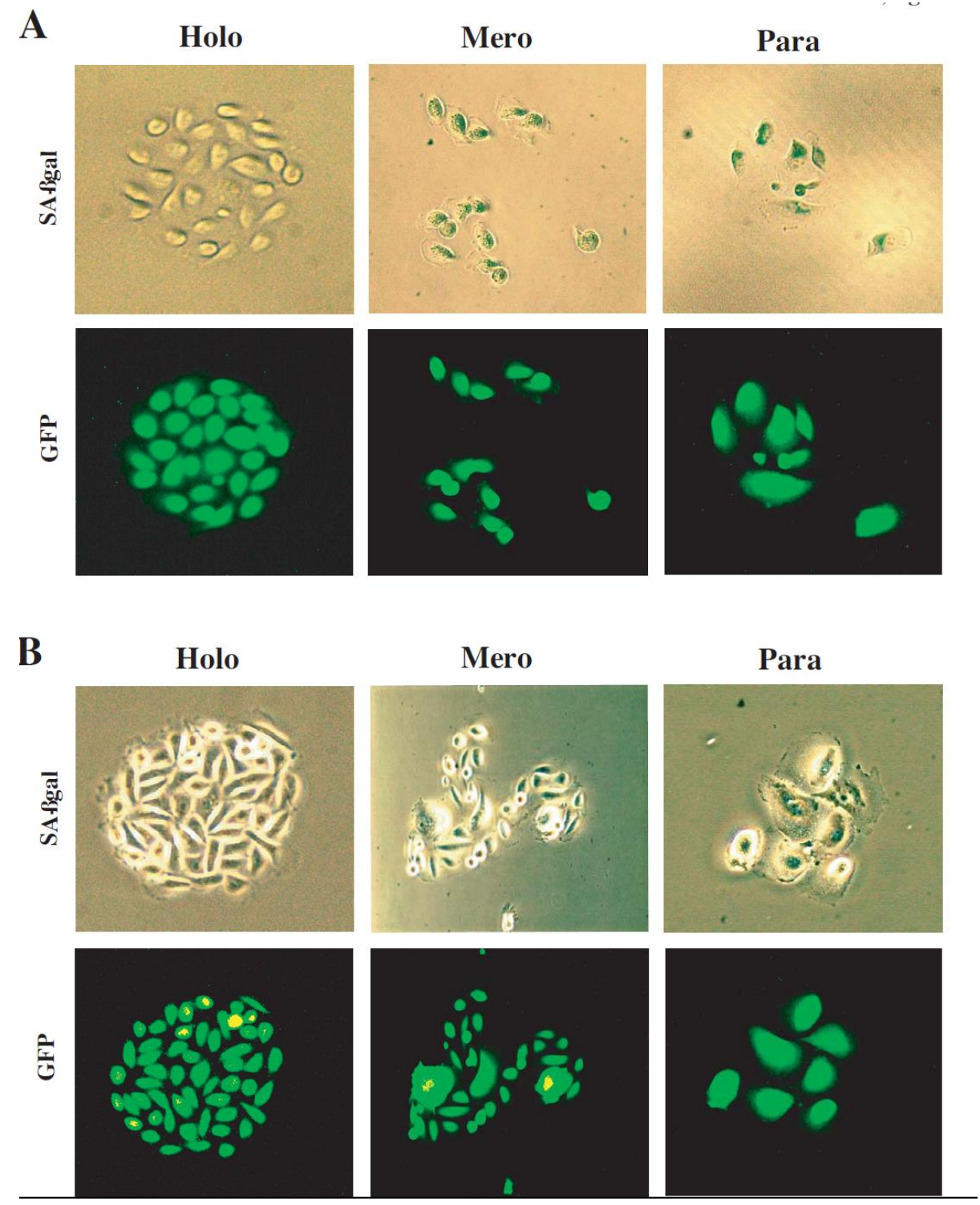

Figure 3.4. Both P1E2 holoclone-derived cells (A) and P1E2 tumor-derived cells (B). when replated, can regenerate the clonal heterogeneity in culture. Original magnifications, x100. 
Table 3.1. Tumorigenicity and serial transplantability of PC3-GFP cell clones

\begin{tabular}{|c|c|c|c|c|}
\hline Clone & Cell & $\begin{array}{c}\text { Tumor } \\
\text { incidence* }\end{array}$ & $\begin{array}{c}\text { Mean } \\
\text { tumor } \\
\text { weight } \\
(\mathrm{g})^{+}\end{array}$ & $\begin{array}{l}\text { Termination } \\
\text { (d) }{ }^{ \pm}\end{array}$ \\
\hline \multirow[t]{4}{*}{ PC3 } & 100 & $1 / 8(13 \%)$ & ND & $90(33)$ \\
\hline & 1,000 & $5 / 8(63 \%)$ & ND & $75(33)$ \\
\hline & 10,000 & $8 / 8(100 \%)$ & ND & $60(18)$ \\
\hline & 100,000 & $7 / 8(88 \%)$ & ND & 45 (18) \\
\hline \multirow[t]{2}{*}{ PC3-GFP } & 10,000 & $7 / 8(88 \%)$ & 1.11 & $65(24)$ \\
\hline & 100,000 & $6 / 8(75 \%)$ & 1.37 & $55(11)$ \\
\hline \multicolumn{5}{|l|}{ P3C4 (holoclone) } \\
\hline & 10,000 & $8 / 8(100 \%)$ & 0.37 & $70(24)$ \\
\hline $2^{\circ}$ & 1,000 & $8 / 10(80 \%)$ & 0.39 & $69(26)$ \\
\hline $3^{\circ}$ & 1,000 & $9 / 10(90 \%)$ & 0.51 & $47(26)$ \\
\hline \multirow[t]{2}{*}{ P1E2 (holoclone) } & 1,000 & $8 / 10(80 \%)$ & 0.21 & $75(54)$ \\
\hline & 10,000 & $9 / 10(90 \%)$ & 1.41 & $75(47)$ \\
\hline $2^{\circ}$ & 1,000 & $5 / 6(83 \%)$ & 0.22 & $55(35)$ \\
\hline $3^{\circ}$ & 1,000 & $7 / 8(88 \%)$ & 0.19 & $49(29)$ \\
\hline \multirow[t]{2}{*}{ P1H8 (holoclone) } & 1,000 & $8 / 10(80 \%)$ & 0.27 & $69(36)$ \\
\hline & 10,000 & $10 / 10(100 \%)$ & 1.39 & $69(28)$ \\
\hline $2^{\circ}$ & 1,000 & $6 / 6(100 \%)$ & 1.06 & 71 (39) \\
\hline \multirow[t]{2}{*}{ P2A12 (meroclone) } & 10,000 & $0 / 10$ & 0 & 187 \\
\hline & 100,000 & $0 / 2$ & 0 & 187 \\
\hline \multirow[t]{2}{*}{ P2H3 (meroclone) } & 10,000 & $0 / 9$ & 0 & 121 \\
\hline & 100,000 & $0 / 2$ & 0 & 121 \\
\hline P2D4 (paraclone) & 1,000 & $0 / 1$ & 0 & 63 \\
\hline
\end{tabular}

NOTE: PC3 or PC3-GFP cells, or PC3-GFP cell clones at the indicated numbers were injected in $50 \%$ Matrigel into the flanks of NOD/SCID mice. Secondary $\left(2^{\circ}\right)$ and tertiary $\left(3^{\circ}\right)$ serial transplantation experiments were performed as described in the text.

Abbreviation: ND, not determined.

*The number of tumors developed/number of injections.

$\dagger$ Mean tumor weight in grams.

‡ Time in days when animals were terminated and the tumor was harvested. The numbers in parentheses are latency (i.e., when tumors were first detected after implantation). 
A
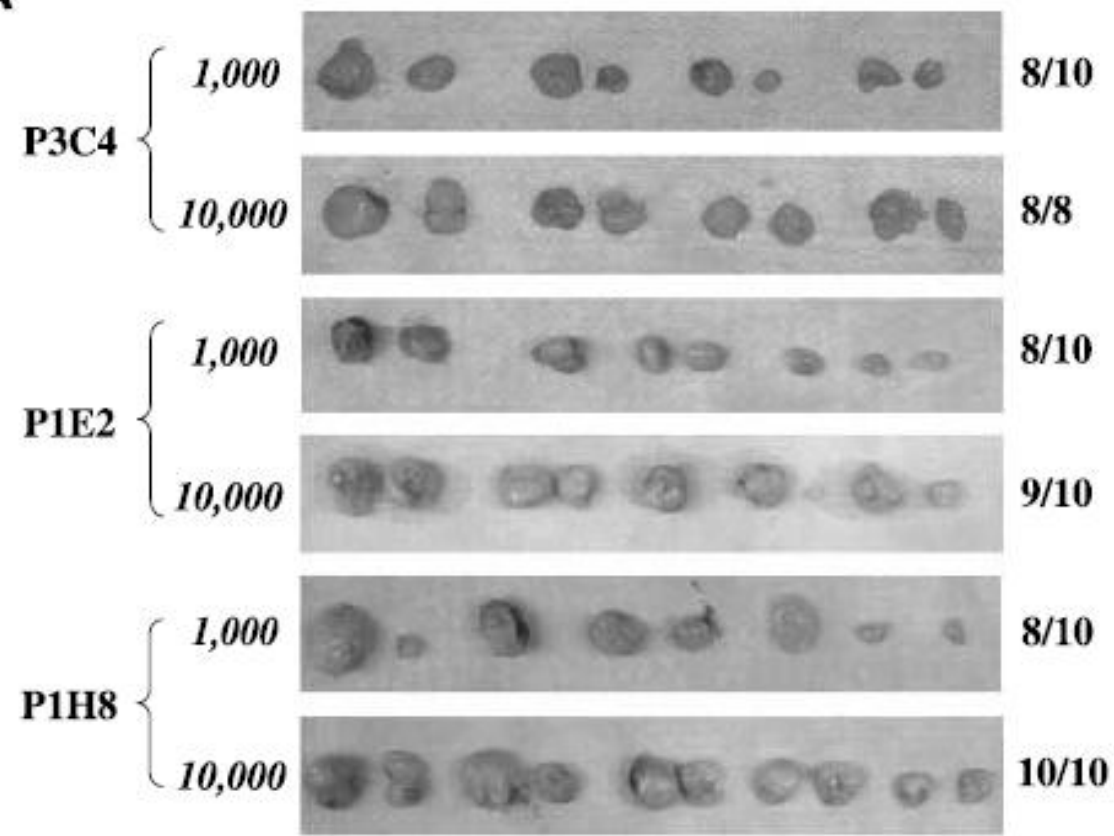

B

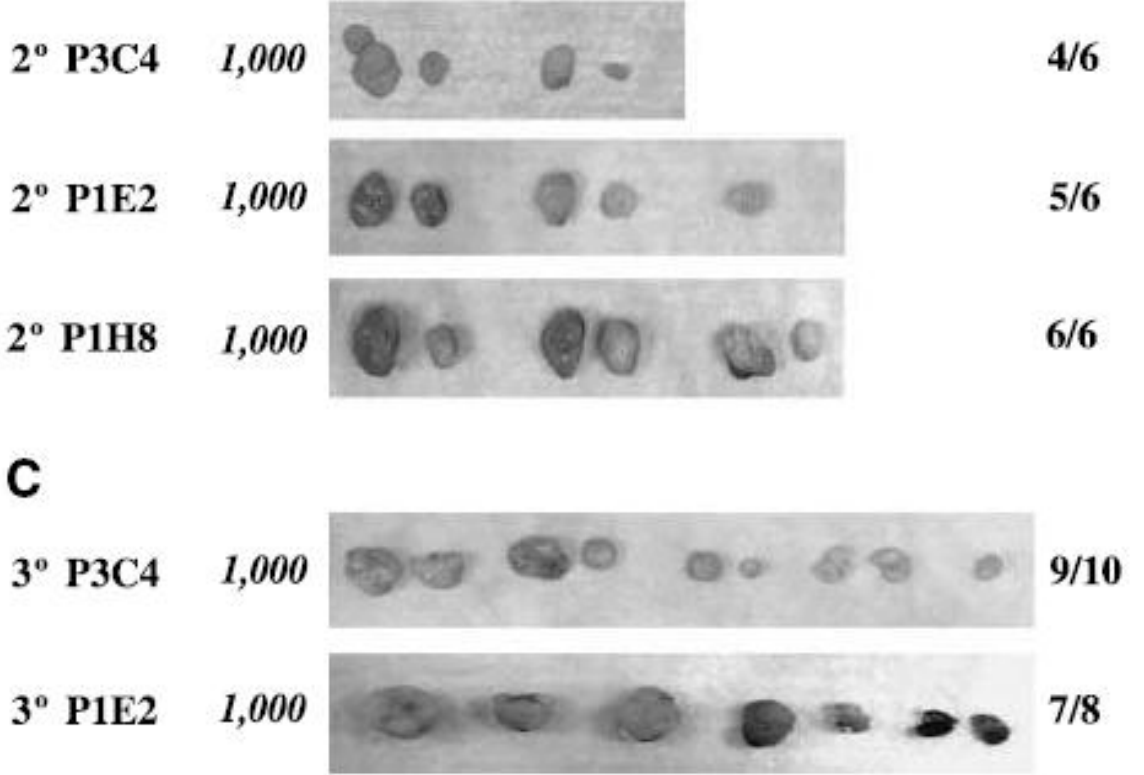

Figure 3.5. Serial tumor transplantation of three holoclone-derived PC3 cells

A to $\mathrm{C}$, tumor images of the first, secondary, and tertiary, respectively, tumor transplants. There are no statistical differences in tumor rates among each clone $(\mathrm{P}>0.05$; Fisher's test). 
paraclones. As shown in Table 1, both PC3 and PC3-GFP cells initiated cell number-dependent tumor development in NOD/SCID mice. As few as 100 PC3 cells initiated tumor development in one of the eight injections and at 10,000 cells most of the injections of either PC3 or PC3-GFP cells developed tumors (Table 3.1). Three holoclones (i.e., P3C4, P1E2, and P1H8), harvested around 6 to 8 weeks after plating, when injected at 1,000 or 10,000 cells, all initiated $80 \%$ to $100 \%$ tumor development in NOD/SCID mice in 47 to 75 days (Table 3.1; Fig. 3.5.A). In sharp contrast, two meroclones (i.e., P2A12 and P2H3) did not develop any tumors in 5 to 6 months even with 10,000 or 100,000 cells (Table 3.1 ). Most paraclones became senescent very early on, and these senescent cells were very difficult to harvest. The only paraclone of which we managed to harvest 1,000 cells did not initiate tumor development (Table 3.1). Importantly, when the three holoclone-derived tumors were harvested and PC3-GFP cells were purified and used in secondary tumor transplantation experiments, 1,000 cells of each clone regenerated tumors in $70 \%$ to $100 \%$ of the injections (Table 3.1 ; Fig. $3.5 \mathrm{~B}$ ).

When tertiary tumor transplantation experiments were carried out with P3C4 and P1E2 secondary tumor-derived cells, 1,000 cells again reinitiated tumor development in $90 \%$ of injections (Table 3.1; Fig. 3.5. C). When the P1E2 holoclone tumor-derived PC3-GFP cells were re-plated in clonal cultures, within one week, all three types of clones were observed (Fig.3.4.B), suggesting that holoclones could regenerate (or maintain) the clonal heterogeneity in vivo. These serial tumor transplantation experiments and re-plating assays provide concrete experimental evidence that PC3 holoclones contain stem-like tumor cells that can initiate serially transplantable tumors. 


\section{Holoclones Express High Levels of Stem and Progenitor Cell Markers CD44, a $2 \beta 1$}

\section{integrin, and $\beta$-catenin}

To further characterize stem cell-associated properties in holoclones, we immunophenotyped bulk-cultured PC3-GFP (Fig. 3.6A) or PC3 (not shown) cells for the expression of three stem and progenitor cell markers-CD44, $\mathrm{a} 2 \beta 1$ and $\beta$-catenin. Our previous work has shown that the $\mathrm{CD}_{4} 4^{+}$and $\mathrm{CD}_{4} 4^{+} / \mathrm{a} 2 \beta 1^{+}$prostate cancer cell populations are enriched in tumorigenic and metastatic CSCs, whereas the $a 2 \beta 1^{+}$prostate cancer cells most likely mark fast-proliferating tumor progenitors (Patrawala et al., 2007; Patrawala et al., 2006). Furthermore, $\beta$-catenin is preferentially expressed in the CD $44^{+}$prostate CSCs (Patrawala et al., 2006). As shown in Fig. 3.6.A, holoclones in bulk-cultured PC3-GFP cells expressed high levels of all three molecules, whereas paraclones showed barely detectable expression of the three markers. Staining of regular PC3 cells (not shown) or holoclone-derived PC3-GFP cultures (Fig. 3.6 B) revealed similarly differential expression patterns of these three stem cell markers. Importantly, meroclone P2H3derived cells, which as expected, did not form holoclones, expressed little CD44 and a2ß1 (Fig. 3.6) or $\beta$-catenin (data not shown). These immunostaining results provide direct experimental evidence that PC3 cell holoclones, but not meroclones and paraclones, contain stem-like cells. The fact that all three markers are expressed in essentially all cells in the holoclones suggests that these three molecules are probably expressed in both cancer stem and progenitor cells. 


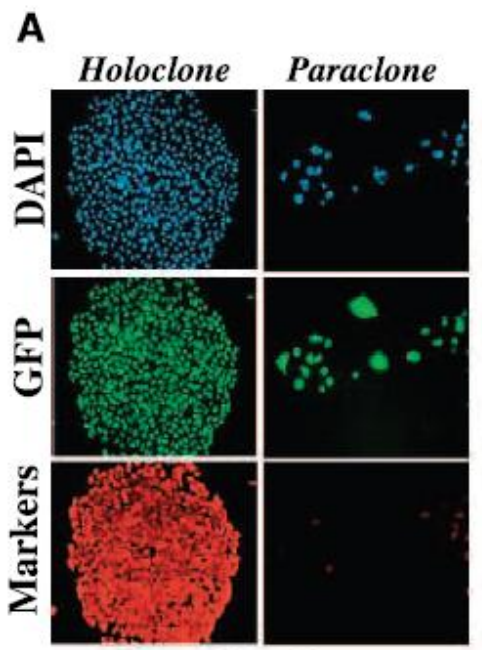

CD44

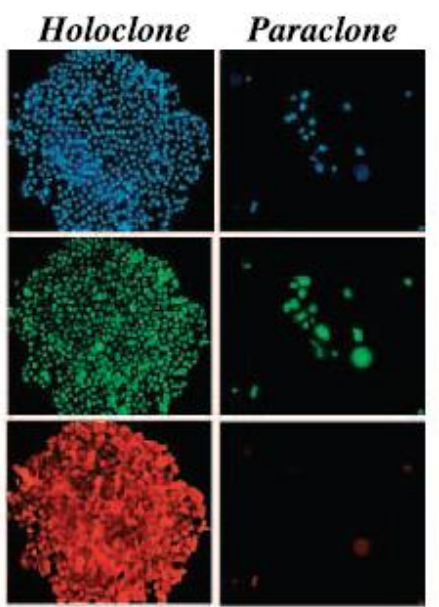

$\alpha_{2} \beta_{1}$

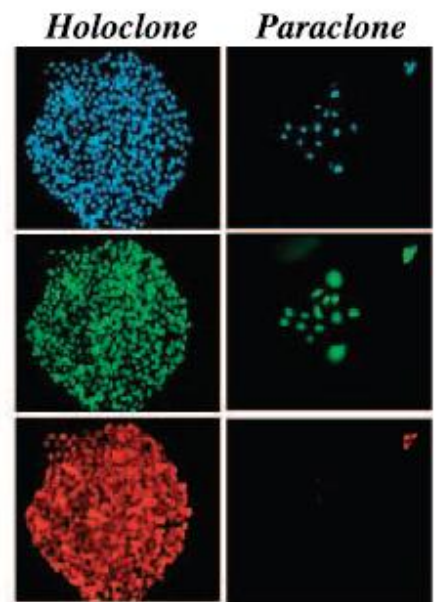

$\beta$-Catenin

C

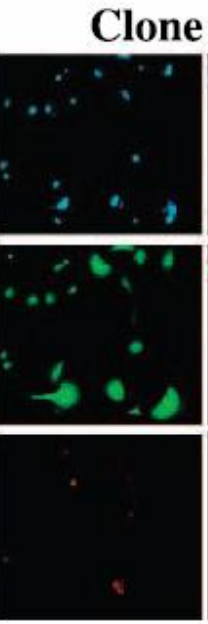

CD44
P2H3
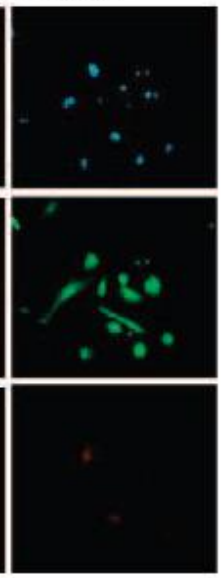

$\alpha_{2} \beta_{1}$

Figure 3.6. PC3 cell holoclones express high levels of stem and progenitor cell markers Bulk-cultured PC3-GFP cells (A), holoclone P1H8 (B), or meroclone P2H3 (C) derived cells were plated on glass coverslips and stained for $\mathrm{CD} 44$, $\alpha 2 \beta 1$, and/or $\beta$-catenin using monoclonal antibodies. Original magnifications, X100. 
In summary, we addressed in this study the critical question of whether epithelial cancer cell holoclones may contain stem-like cells. Through clonal analyses, serial passaging in vitro, serial tumor transplantation experiments, re-plating assays, and immunophenotyping, we provide unequivocal evidence that PC3 prostate cancer cell holoclones contain self-renewing cancer cells that could initiate serially transplantable tumors. Because tumor regeneration in serial transplantation experiments is the current gold standard for defining CSCs (Clarke et al., 2006; Tang et al., 2007), our results suggest that PC3 holoclones contain putative CSCs. In support, PC3 cell holoclones, but not meroclones and paraclones, express the three well-established stem and progenitor cell markers mentioned above. This suggestion is also consistent with primary keratinocyte holoclones containing normal stem cells (Barrandon and Green, 1985; Barrandon and Green, 1987; Claudinot et al., 2005). The observations made herein are unlikely restricted to only PC3 cells as Du145 holoclones can also be serially passaged and sustain long-term propagation (Tang et al., 2007) and many other epithelial cancer cells form holoclones that also express CSC markers such as CD44 (Locke et al., 2005). Future work will focus on devising ways to enrich for stem-like cells in cancer cell holoclones and then prospectively purifying them and characterizing their biological properties. 


\section{Chapter IV: Microenvironment-Regulated Manifestation of Metastatic Prostate CSCs}

\section{Introduction}

The CSC hypothesis proposes many similarities between normal SCs and CSCs. The CSCs may be a small population that expresses normal SC markers, and may undergo asymmetric division (Reya et al., 2001). Evidence suggests that CSCs have the ability to reinitiate serially transplantable tumors (Li et al., 2009; Shackleton et al., 2009). The SC niche plays a critically important role in maintaining SC properties and preventing depletion of SCs and may further endow additional functions to SCs. Compared with the SC niche, the tumor microenvironment is also proven to serve a significant role to modulate tumor growth, progression and metastasis (Fidler et al., 2007).

A fundamental unanswered question is whether or not this tumor microenvironment behaves like the niche? Tumor microenvironment that comprised non-malignant stromal cells, inflammatory cells fibroblasts, soluble factors, vascular networks, nutrients and metabolic components, and the structural extracellular matrix (ECM) components have been better characterized than that of metastatic sites and has been shown to promote tumor metastasis (Fidler et al., 2007). The metastatic niche model suggests that only those primary tumors with a pre-metastatic niche that is characterized by an inflammatory microenvironment allow tumor cells to disseminate and proliferate at the secondary site (Peinado et al., 2008; Psaila and Lyden, 2009). In addition, the CSC hypothesis would not be complete without a link to metastasis; human tumors at advanced stages (such as Gleason grade 9-10 PCa) may also contain subsets of cells that have already been bestowed with the ability to disseminate and metastasize (An et al., 
1998). Therefore, the ideal 'reconstitution' system should also allow the manifestation of the metastatic potential of these rare cells. Putative breast CSCs, isolated mostly from metastases, were shown to possess high tumor-reinitiating ability without showing increased metastatic potential (Sheridan et al., 2006), presumably reflecting an inadequate microenvironment in the "reconstitution" system to recapitulate the metastatic propensity of the presumptive CSCs (Hurt and Farrar, 2008; Sheridan et al., 2006). Hence, the relationship between CSCs and metastatic niche is the next urgent question. Not many reported studies have specifically addressed this question. The CSC model may be the best to answer this question since the CSC perhaps possess the intrinsic ability to metastasize.

Clinically, PCa metastasizes to lymph nodes (LN), bone, lung, liver, pancreas, and some other organs with a preference for the bone, suggesting that the end-organ microenvironment has a great influence on the route and pattern of PCa cell dissemination (Morrissey and Vessella, 2007). Experimentally, metastasis is most frequently studied by implanting human PCa cells in different sites of recipient mice followed by observation of pattern/extent of disseminating tumor cells. It has long been observed that various human cancer cells implanted into an orthotopic organ environment in mice display much more widespread and extensive metastasis than tumor cells implanted at an ectopic site (Fidler, 2002; Fidler et al., 2007; Langley and Fidler, 2007). This widely-established phenomenon suggests that the microenvironment in which human cancer cells are implanted also greatly influences the manifestation of their metastatic potential in recipient mice. For example, Dr. Fidler's group reported in 1992 that in an experimental mouse model, PC3-M cells implanted in an orthotopic site (i.e., prostate) led to widespread metastasis whereas the same tumor cells implanted in an ectopic site such as subcutaneous (s.c), though 
showing similar tumor development, failed to give rise to significant metastasis (Stephenson et al., 1992). Many subsequent studies (An et al., 1998; Jantscheff et al., 2009; Rembrink et al., 1997; van Weerden and Romijn, 2000; Wang et al., 2005a; Wang et al., 2005b; Yang et al., 1999), using similar and improved animal models, provide confirmation of the essential role of the 'orthotopic' environment in reconstituting the metastatic capacity of human (prostate) cancer cells. The clinical (Morrissey and Vessella, 2007) and experimental (Fidler, 2002; Hoffman, 2002; Langley and Fidler, 2007) observations, taken together, imply that the mouse prostatic microenvironment resembles, at least partly, the human prostate microenvironment. Despite these important and long-held observations, what factors in the orthotopic microenvironment and what microenvironment-affected genes in $\mathrm{PCa}$ cells which promote the manifestation of metastatic potential remain scarcely understood.

Genomic microarray has been widely applied in tumor microenvironment field (van 't Veer et al., 2002). Gene expression profiles identified by these studies are applied either for mechanismal studies or for clinical related diagnosis and prognosis. Recent large scale genetic microarray studies on human breast cancer and prostate cancer indicated that tumor stroma, or tumor microenvironment, is correlated to tumor progression, angiogenesis, and metastasis (Allinen et al., 2004; Dakhova et al., 2009; Dhanasekaran et al., 2001; Foye and Febbo; Hu and Polyak, 2008b; Ma et al., 2009; Patocs et al., 2007; Richardson et al., 2007; Tomlins et al., 2007; True et al., 2006; Varambally et al., 2005). However, most of these gene signature studies used a single model and it may be generally difficult to clearly distinguish the epithelial from the stromal parts with the current microdissection techniques. Hence, human xenograft model may be an ideal model to study interaction between epithelial and host stroma in that human cancer 
cells can be distinguished from mouse stromal cells by using human and mouse microarray, respectively. In addition, there have not been many microarray studies applied to CSC and their regulation by tumor microenvironment. Though a CSCs gene signature has been associated with metastasis and malignance, many functional assays were not performed in this study (Ben-Porath et al., 2008). Whether or not these stemness genes lead or contribute to metastasis is still uncertain.

Herein, in order to address these microenvironment-related questions, we set up orthotopic and ectopic PCa xenograft models with different metastatic profiles; we also performed both human and mouse gene microarray analysis by comparing these two types of tumors and identified a significant CSCs metastasis gene signature. We further purified a metastatic CSC population and show that this population significantly regulates metastasis. These data provide insight into understanding of the CSC niche and metastatic CSCs during PCa progression.

\section{Materials and Methods}

Cells, reagents, and animals Several metastatic PCa cell lines and xenografts (Li et al., 2008; Patrawala et al., 2007; Patrawala et al., 2006; Patrawala et al., 2005) were used in the current study, including PC3 and LAPC9 (derived from bone metastases), LNCaP and LAPC4 (derived from LN metastasis), and Du145 (derived from brain metastasis). LNCaP, Du145, and PC3 cells,

or their GFP-labeled derivatives ( $\mathrm{Li}$ et al., 2008), were cultured in RPMI 1640 medium containing $7 \%$ of heat-inactivated fetal bovine serum (FBS). Xenograft human prostate tumors LAPC-4 and LAPC-9 were courtesy of Dr. C. Sawyers (Tsingotjidou et al., 2001) and were 
maintained in NOD/SCID mice. Du145 xenograft tumors were established using early-passage cells and maintained in NOD/SCID mice. GFP-(or RFP) labeled xenograft tumors (i.e., Du145, LAPC4, and LAPC9) were established by infecting freshly purified tumor cells with a lentiviral vector encoding GFP (or RFP) followed by transplanting FACS-purified tumor cells into NOD/SCID mice. All animals were obtained from Jackson Laboratories and were maintained in standard conditions according to institutional guidelines. The isotype control antibody and FITC, PE, or AlexaFluor-conjugated secondary antibodies were from Chemicon. All chemicals were obtained from Sigma unless specified otherwise.

\section{Analysis of spontaneous metastasis}

Potential metastasis was first monitored in animals by symptoms such as hunched posture, irregular breathing and gait, and paraplegia. When systemic symptoms and/or primary tumor burden became obvious or the tumor-bearing animals became moribund, animals were sacrificed by $\mathrm{CO} 2$ euthanization and cervical dislocation. Necropsy was performed to isolate individual organs and to visually examine them for gross metastases (i.e., metastatic nodules, metastatic adhesions, etc). The presence of metastases in each organ was first screened under a wholemount epifluorescence Stereo Microscope (Nikon SMZ1500) with a DXM1200F digital camera and imaging software. Then individual organs were dissected and, after thorough rinsing, subjected to collagenase (type I) digestion $\left(37^{\circ} \mathrm{C} ; 60 \mathrm{~min}\right)$ followed by trypsin $\left(0.25 \% ; 37^{\circ} \mathrm{C} ; 8-\right.$ 15 min) digestion. The resulting single-cell suspension and small tissue pieces from the whole organ were plated on a collagen-coated culture dish in RPMI1640 plus 8\% FBS. The presence or absence and the relative abundance of metastatic, $\mathrm{GFP}^{+} \mathrm{PCa}$ cells in different end organs were 
independently screened and semi-quantified by two individuals under an Olympus BX50 fluorescence microscope. The detection sensitivity was up to $1 \mathrm{GFP}^{+}$cell. Then cells were cultured in RPMI-FBS medium. In some experiments, the expanded PCa cells derived from metastatic sites were purified using FACS and used in secondary tumor and metastasis assays.

\section{cDNA microarray analysis}

Total RNA was extracted from tumor pieces by using an RNeasy RNA-purification kit (Qiagen, Valencia, CA), including on-column DNase digestion to completely remove contaminating genomic DNA. RNA concentrations were determined using a NanoDrop and the quality assessed using a BioAnalyzer. Microarray experiments were carried out using the 44k 60-mer "Human Whole Genome Oligo Microarray Kit" from Agilent (Agilent Technologies, Alto, CA) with 500 ng of total RNA as starting material according to the manufacturer's protocol. This array is specific for and only detects human genes. 200-500 ng of total RNA was used for each sample in cRNA synthesis. SC samples were labeled with Cy3 and DP samples were labeled with Cy5. The DP and SC samples from the same animal were hybridized in triplicate (i.e., there were a total of 9 hybridizations) and the arrays were scanned with Agilent's dual-laser based scanner. Feature Extraction software GE2-v4_91 was used to link a feature to a design file and determine the relative fluorescence intensity between the two samples (Append. II). Each comparison (i.e., DP1 vs SC1, DP2 vs SC2, DP3 vs SC3) was first analyzed individually and data was combined later as the samples were biological triplicates. Using the normalized arrays, fold-change values were computed for each one of the comparisons and the probe sets showing a $\geqslant 2$ fold change were identified. A mean fold change value for each gene (i.e., DP1/DP2/DP3 vs SC1/SC2/SC3) 
was computed and the two aggregate groups were compared by means of paired t-test and the associated p-values were obtained. Therefore, a total of 21 G4112F-014850 Agilent microarrays (each array containing 45,015 probe sets) were used. Hybridization was performed at $65^{\circ} \mathrm{C}$ for 17 hrs and hybridized arrays were scanned with Agilent's dual-laser based scanner. Feature Extraction software GE2-v5_91 was used to link a feature to a design file and determine the relative fluorescent intensity between the two samples.

We used the lowess-normalized data provided with the data files (columns gDyeNormSignal and rDyeNormSignal), using the annotation provided by Agilent (http://www.chem.agilent.com/cag/bsp/gene_lists.asp?arrayType=gene).We focused on the 18,841 distinct gene symbols with each symbol corresponding to one or more probe sets. In the case of multiple probe sets per gene, they were treated as additional replicates for the analysis. For each one of the 18,841 genes, we identified the corresponding probe sets (one or more) and applied a paired t-test between the group of interest and the control (sample \#1). The paired t-test yields t-statistic values as well as p-values to assess the statistical significance of differential expression. For each comparison between the group of interest and control, a beta-uniform analysis (BUM) was performed on the corresponding p-values (Welsh et al., 2001) to control for the false discovery rate (fdr) that is typically used to account for multiple testing in high throughput data. In this case, we used a rather small fdr value, equal to 0.0001 . All comparisons yielded a varying and large number of significant genes.

\section{Hierarchical Clustering Analysis}


The genes were ranked based on their fold change. Either complete list of up and down regulated genes or the top 100 (50) up and down regulated genes in human and mouse arrays were selected for clustering analysis. Two-way clustering was then carried out on these gene expression values in 9 mouse/human arrays, with Euclidean distance and average linkage. All the analyses were performed using R (Version 2.10.0).

\section{Meta-analysis}

Nine prostate cancer datasets (Figure A in 4.30) with primary and metastasis prostate tumors were downloaded from 'Oncomine' (Compendia Biosciences, Ann Arbor, MI). Of all the differentially expressed genes in DPvSSQ comparisons (human), 429 up-regulated and 114 down-regulated genes are detected in at least one dataset. If a gene were significantly upregulated $(\mathrm{p}<0.05)$ in at least 3 datasets, it was arbitrarily set as a consensus up-regulated gene in prostate cancer metastasis. The same method was applied for the down-regulated genes.

\section{Ingenuity Pathway Analysis (IPA)}

We used an online tool based on a curated database, the Ingenuity Pathways Knowledge Base (IPKB), to annotate genes and to determine potential regulatory networks and pathways. The IPKB contains information on human, mouse, and rat genes including annotations, synonyms and over 1.4 million published biological interactions between genes, proteins and drugs. This database is continually updated and supplemented with curated relationships taken from MEDLINE abstracts; i.e. each gene interaction held in the IPKB is supported by published information. IPKB thus provides a framework by which lists of genes identified by large scale 
microarray studies can be annotated in terms of their functional relationships, and those that have been shown to interact.

\section{Gene Ontology}

Gene ontology (GO) analysis was carried out using Gene Ontology Analysis module from ArrayTrack microarray analysis suite developed by (food and drug administration) FDA's NCTR (Fang et al., 2009) and also by using WebGestalt (WEB-based GEne SeT AnaLysis Toolkit) developed and maintained by the bioinformatics resource center at Vanderbilt. When a gene was associated with multiple GO terms, gene card from NCBI was used to assign the gene to the appropriate GO term based on its functions.

\section{Purification of human PCa cells from xenograft tumors}

Basic procedures have been previously described (Li et al., 2008). Briefly, Du145, LAPC-4, and LAPC-9 xenograft tumors (either regular or GFP-tagged, see below) were aseptically dissected out from animals and minced into $\sim 1 \mathrm{~mm} 3$ pieces in DMEM or RPMI supplemented with $10 \%$ FBS. After rinsing in the same medium (2x), tumor tissues were incubated with 1x Accumax $(1,200-2,000 \mathrm{U} / \mathrm{ml}$ proteolytic activity containing collagenase and DNase; Innovative Cell Technologies, Inc, San Diego, CA) at $10 \mathrm{ml} / 1 \mathrm{~g}$ tissue in DPBS for $\sim 30 \mathrm{~min}$ at room temperature under rotating conditions. A single cell suspension was obtained by filtering the supernatant through a 40- u m cell strainer and the cell suspension was then gently loaded onto a layer of Histopaque-1077 gradient ( $1-3 \times 106$ cells $/ \mathrm{ml}$ HistoPaque in a total of $3 \mathrm{ml}$ volume) and then centrifuged at $400 \mathrm{~g}$ for $30 \mathrm{~min}$ at room temperature. Red blood cells, dead cells, and debris were eliminated from the bottom of the tube and live nucleated cells were collected at the interface. The resultant cell mixture was depleted of lineage-positive host cells using the MACS Lineage 
Cell Depletion Kit (Miltenyi Biotec, Auburn, CA). Briefly, cells were first incubated (10 min at $4^{\circ} \mathrm{C}$ ) in the staining solution (PBS, $\mathrm{pH} 7.2,0.5 \% \mathrm{FBS}, 0.5 \mathrm{ug} / \mathrm{ml}$ insulin) containing biotinylated antibodies against a panel of lineage antigens (CD5, CD45R, CD11b, anti-Ly-6G, 7-4, and Ter119). Cells were then incubated with anti-biotin Microbeads $\left(15 \mathrm{~min}\right.$ at $\left.4^{\circ} \mathrm{C}\right)$ and the Lin- cells were eluted using the MS columns. The eluted PCa cells were all human epithelial cells as confirmed by their expression of Ber-EP4, a surface marker unique to human epithelial cells.

\section{Purification of human PCa cells from primary tumors}

The basic protocol is modified from the above xenograft tumor protocols (Li et al., 2009). Briefly, patient tumors were minced into $\sim 1 \mathrm{~mm} 3$ pieces and tissues are subjected to enzymatic digestion (type I collagenase plus DNase at $50 \mathrm{U} / \mathrm{ml} 4 \mathrm{~h}$ to overnight, depending on the size and composition of tumors). Upon digestion, epithelial organoids are enriched by a brief centrifugation followed by trypsin digestion to release epithelial cells. Samples are then subjected to a discontinuous Percoll gradient purification step to remove the majority of mononuclear blood cells and dead cells. Finally, the cell preparation is subjected to a negative selection process using the MACS and the antibody cocktail (anti-CD3, 14, 16, 19, 20, 45, 56, and $140 \mathrm{~b}$ ) to remove the $\mathrm{Lin}^{+}$cells including hematopoietic, endothelial, and other stromal cells (smooth muscle, myoepithelial, fibroblast, etc). This digestion/purification procedure takes 6-8 h to accomplish and generally results in $>98 \%$ pure human epithelial cells as judged by Ber-EP4 staining. Purified human PCa cells are resuspended in IMDM-20\% FBS for use. 
Flow-Activated Cell Sorting (FACS) Based on Cell Surface Markers, Side Population Phenotype, or GFP Expression

Basic procedures have been described previously (Patrawala et al., 2007; Patrawala et al., 2006; Patrawala et al., 2005). The $\mathrm{GFP}^{+}$metastatic tumor cells ( LM1\&BM1) were sorted using a flow cytometer (Coulter) from culture of distant metastatic organ and used for secondary enrichment metastatic injection. Sorting by cell surface markers OPN, CD24, CD44, CXCR4, ABCG2 and CD133; and side population phenotype were performed as previously described (Patrawala et al., 2007; Patrawala et al., 2006), and sorted cells directly used for tumor implantation experiment or RNA extraction for Real-time qRT-PCR.

\section{s.c tumor cell injection}

Basic procedures have been previously described (Li et al., 2008). Briefly, NOD/SCID mice (male, 6-8 week old) were injected s.c. with different cell numbers in 50ul of medium containing $\sim 50 \%$ Matrigel. Tumor development was monitored starting from the second week. Tumorigenicity was measured mainly by tumor incidence (i.e., the number of tumors/number of injections), latency (i.e., time from injection to detection of palpable tumors), and tumor weight. All animals were terminated at 6-9 months after tumor cell injection. Tumors were fixed in formalin and paraffin sections were made for H\&E staining and immunohistochemistry.

\section{Dorsal prostate (DP) implantation}

Basic procedures have been previously described (Li et al., 2009; Patrawala et al., 2005). Briefly, male NOD/SCID mice (6-8 weeks) were anesthetized by ketamine/xylazine (65 mg/kg 
body weight) and isoflurane as needed in supine position. A lower horizontal abdominal incision was made, the bladder and seminal vesicles were gently pulled out to expose the DP, and then a total of 20ul cell suspension (10ul cell-containing medium mixed with 10ul Matrigel) was injected into the DP using a 30-gauge Hamilton syringe. Then the organs were gently put back into the body cavity and the surgical wound was closed in two layers with 4-0 Dexon interrupted sutures. The abdominal incision was closed in one layer by using surgical staples. All procedures were performed under a dissecting light microscope.

\section{Anterior prostate (AP) implantation}

PCa cells at different numbers were first mixed with type I rat collagen and incubated in a tissue culture plate at $37^{\circ} \mathrm{C}$ for $10-15 \mathrm{~min}$. Then the solidified cell pellets were gently covered in medium and cultured for $4 \mathrm{hrs}$ to overnight prior to implantation. For AP implantation (Stephenson et al., 1992; Wang et al., 2005a; Wang et al., 2005b), a transverse incision was made in the lower abdomen to expose the AP by partially pulling the bladder, seminal vesicles and prostate out of the abdominal cavity. A 2-3 mm incision was made in the AP through the tubule between the two main ducts with the aid of a 22-gauge needle. Using a fire-rounded glass pipette tip, the collagen dots were inserted into a pocket formed under the prostate tubule. Then the organs were replaced and the body wall and skin closed. In some experiments, tumor pieces of $\sim 2-3 \mathrm{~mm}$ were implanted using a sterile trocar.

\section{Cell recombination with rat urogenital sinus mesenchyme (rUGM) followed by kidney capsule (KC) transplantation (the $\mathrm{rUGM} / \mathrm{KC}$ protocol)}


Basic procedures have been described (Staack et al., 2003). The whole procedure of KC grafting can be found in an illustrated tutorial at http://mammary.nih.gov/tools/mousework/Cunha 001/index.html. Briefly, the UGM was first isolated from E17 - E18 rat embryos. PCa cells at various numbers were then mixed with different portions of rUGM using a rough ratio of one rUGM per 200,000 PCa cells. Then tissue recombinants were made in collagen as described above and incubated overnight prior to transplantation. For KC transplantation, the host NOD/SCID mice were routinely supplemented with testosterone pellet and generally one kidney received the transplantation. Grafts were collected and analyzed 2 - 5 months later.

\section{Immunofluorescence staining and Western blotting}

Basic procedures have been described (Bhatia et al., 2003; Chandra et al., 2006; Chandra et al., 2004). For surface proteins, cells were fixed in $4 \%$ paraformaldehyde (PFA) and then directly used in immunolabeling. For cytosolic proteins, cells were first permeabilized with $1 \%$ Triton X100 and then used in immunostaining. For nuclear proteins, cells were stained as for cytosolic proteins except that $0.5 \%$ Triton $\mathrm{X}-100$ was included in the primary and secondary antibody solutions. For cytoskeletal proteins (i.e., CK5 and CK18), cells were fixed and permeabilized in methanol/acetone $\left(1: 1 ;-20^{\circ} \mathrm{C}\right)$ for $10 \mathrm{~min}$ and then used in immunostaining. Cells were both analyzed for fluorescence intensity and quantified for the percentage of positive cells. For the latter, $600-1,200$ cells were counted for each condition and statistical analyses were performed using the Student's t-test. 


\section{Immunohistochemical (IHC) staining of paraffin-embedded sections or cryosections}

For IHC, formalin fixed, paraffin-embedded tissue sections $(5 \mathrm{~mm})$ were deparaffinized and hydrated. Endogenous peroxidase activity was blocked with 3\% $\mathrm{H} 2 \mathrm{O} 2$ in water for $10 \mathrm{~min}$. Antigen retrieval was performed with $10 \mathrm{mM}$ citrate buffer ( $\mathrm{pH}$ 6.0) for $10 \mathrm{~min}$ in a microwave oven followed by a 20-minute cool down and thorough wash. These treated slides or cryosections $(8 \mathrm{~mm}$ ) were incubated with Biocare Blocking Reagent (\#BS966M with casein in the buffer; Biocare, Concord, CA) for 10 min to block non-specific antibody binding. After draining, slides were incubated with various primary antibodies (at $100-2,000$ dilutions as determined from pilot experiments) for 30 minutes at room temperature. Slides were washed in phosphate buffer twice and then incubated in biotinylated goat-anti-rabbit or mouse IgG (Vector Laboratories, Burlingame, CA) at a 1:500 dilution for $30 \mathrm{~min}$ at RT. After thorough washing, slides were incubated with SA-HRP (BioGenex Laboratories Inc., San Ramon, CA) for 30 minutes at room temperature followed by washing. Finally, slides were incubated with BioGenex DAB substrate and color development was closely monitored under a microscope. In most cases, slides were lightly counterstained with hematoxylin. For cytoskeletal protein (e.g., CK5 and CK8) staining, slides were treated with proteinase $\mathrm{K}(0.06 \%)$ for $20 \mathrm{~min}$ prior to blocking.

\section{Lentiviral Production and Transduction of Cancer Cells}

The pLL3.7 lentiviral vectors have been previously described (Li et al., 2008; Zaehres et al., 2005). The pCMV-DsRed-Expression 2 vector ( Clontech, Mountain view, CA) is a lentiviral vector that is expressed in mammalian cells and consistently express red fluorescent protein for whole cell labeling or as a marker for construct transfection. pGIPZ constructs have been 
previously described (Jeter et al., 2009), and were obtained from Open Biosystems (Open Biosystems, Huntsville, AL), and included the non-silencing verified (shRNA expressing) pGIPZ-negative control vector (cat\# RHS4346), pGIPZ-OPN,MMP9,CD24,TGF $\beta$ 1,TGF $\beta$ 2,IL-1 $\beta$,ESM1. For LL3.7 and TRC constructs, lentivirus was produced in 293FT packaging cells (obtained from Clontech) using modified protocols previously described (Zaehres et al., 2005). In brief, geneticin selected, early-passage $293 \mathrm{FT}$ cells $(6$ million $/ 15 \mathrm{~cm}$ dish $)$ were transfected with the RRE (6 mg), REV (4 mg) and VSVg (4 mg) packaging plasmids, along with a lentiviral vector $(6 \mathrm{mg})$ using Fugene 6 at a 1:2.7 ratio of DNA $(\mathrm{mg})$ to transfection reagent (ml). At 36-48 h, media containing virus were collected and fresh media added. After an additional 12-24 $\mathrm{h}$ of culture, viral supernatants were again collected, pooled, and ultracentrifuged to produce concentrated viral stocks. Individual titers were determined for the GFP-tagged virus using HT1080 cells. pGIPZ constructs were similarly packaged into lentivirus using 293T cells according to the Trans-Lentiviral Packaging system (cat\# TLP4615; Open Biosystems, Huntsville, AL). Viral containing media was collected and ultracentrifuged, and the titer determined, as described above. Cells were plated $24 \mathrm{~h}$ earlier and infected at approximately $50 \%$ cell density. Cells were harvested for in vitro or in vivo characterization 48 $72 \mathrm{~h}$ post-infection.. Aggregates were gently dissociated by a brief digestion in Accumax prior to injection. For primary tumor-derived cells, tumor cells and viruses were plated together on collagen-coated dishes. After infection, cells were dissociated by Trypsin/EDTA, followed by Trypsin Neutralization Solution. The resultant single-cell suspensions were replated in PrEBM or used for tumor transplantation studies as described above. 


\section{Real-time quantitative RT-PCR analysis}

Basic procedures have been previously described (Jeter et al., 2009). Briefly, total RNA was extracted from cancer cells by using an RNeasy RNA-purification kit (Qiagen, Valencia, CA, http://www1.qiagen.com). RT-PCR was performed using an ABI Prism 7900HT and the TaqMan system (ABI; Applied Biosystems, Foster City, CA, http://www.appliedbiosystems.com).

\section{Invasion and migration assays}

The original assay were developed by McEwan's group (Albini et al., 1987), the Biocoat Matrigel invasion chamber was purchased from Becton Dickinson (Bedford, MA), which consists of an 8- $\mu \mathrm{m}$ pore size polyethylene terephthalate (PET) membrane that has been overlaid with Matrigel (basement membrane matrix). PC3,C-4-2, PC3-GIPZ-MMP9 cells were suspended at $2.5 \times 10^{3}$ cells $/ 0.25 \mathrm{ml}$ in serum-free media, then were added to the upper chamber. Next, 0.5 $\mathrm{ml}$ media with $15 \%$ serum added to the lower chamber. The chambers were incubated for $22 \mathrm{~h}$ at $37^{\circ} \mathrm{C}$ in a humid atmosphere of $5 \% \mathrm{CO} 2$. After incubation, the filters were fixed ( we did not stain the filters since our cells are all GFP labeled)and the upper surface of the filters was scraped twice with cotton swabs to remove non-invading cells. The experiments were repeated in triplicate wells, and the number of invading cells in ten high-power fields per filter was counted microscopically at $\times 400$ magnifications. The data were processed by the method described in migration assay.

\section{Statistical Analysis}


All statistical analyses were performed in SigmaPlot using unpaired Student's t-test, ANOVA (F-test), or $x^{2}$ test, depending on the nature of comparisons and type of data.

\section{Results and Discussion}

\section{Human PCa Cells Implanted Into The Mouse Prostate Demonstrate More Widespread Metastasis than Cells Implanted at Ectopic Sites.}

The orthotopic tumor xenotransplantation model has played an important role in understanding human cancer metastasis (Kang, 2009). It is the xenograft model that most closely mimics the process of human cancer metastasis, probably because the primary tumor grows up in a similar microenvironment to that of the human tumor and thus produces spontaneous metastasis. Subcutaneous implantation is a widely-used xenograft ectopic model in cancer research due to its ease of handling and low cost. Most recent CSC studies use the s.c. injections as the primary method of growing tumors. However, when compared the metastatic profiles between orthotopic tumor and s.c. tumor, Dr. Fidler's group first indicated that orthotopic models always produce more metastasis than sc models in PCa (Stephenson et al., 1992). One of the most direct conjectures for this interesting phenomenon is about angiogenesis. Surprising, Dr. Jain and Dr. Fukumura' $\mathrm{s}$ works did not show consistently that orthotopic microenvironment promoted vascular endothelial growth factor/ vascular permeability factor (VEGF/VPF) increasing compared with s.c. microenvironment. In pancreas tumor model, there are more VEGF in orthotopic tumors than that of s.c. tumors (Tsuzuki et al., 2001); but in colon cancer and breast cancer model, the results were reversed that there were more VEGF expression in s.c. tumors (Fukumura et al., 1997; Monsky et al., 2002). There was no difference in the expression of 
VEGF between orthotopic and s.c. tumors in gallbladder tumor model (Gohongi et al., 1999). Fidler's subsequent work revealed a few metastasis-related genes upregulated in the orthotopic tumors versus s.c. tumor in colon and renal tumor models (Kitadai et al., 1995; Morikawa et al., 1988), a full molecular mechanism underlying this highly metastatic orthotopic microenvironments is still lacking.

In the PCa field, there has not been a comprehensive study comparing tumorigenic and metastatic ability of human PCa cells implanted at different sites in the recipient mice. Our preliminary data in two distinct type of PCa: PC3 (Figure 4.1) and LAPC9 (Figure 4.2) demonstrated that DP microenvironment allows more metastasis profiles than the s.c. site. In order to further determine how transplantation sites/microenvironments may affect PCa metastasis, we did a comprehensive study that not only looks at the metastatic difference between these sites, but also the difference in tumor growth (table 4.1, and figure 4.3 B, C).

Since the PC3 line is an AR-independent, PSA negative line, we also used other prostate cancer lines that are AR dependent and PSA positive in the same experiment. Finally, we choose PC3, DU145, LAPC9, LAPC4 and LNCaP as a representative for PCa line. This will allow us to find some common mechanism in the whole PCa fields. We injected different cell number according limited dilution into these two sites (DP vs s.c.) by using these five PCa lines. 
A
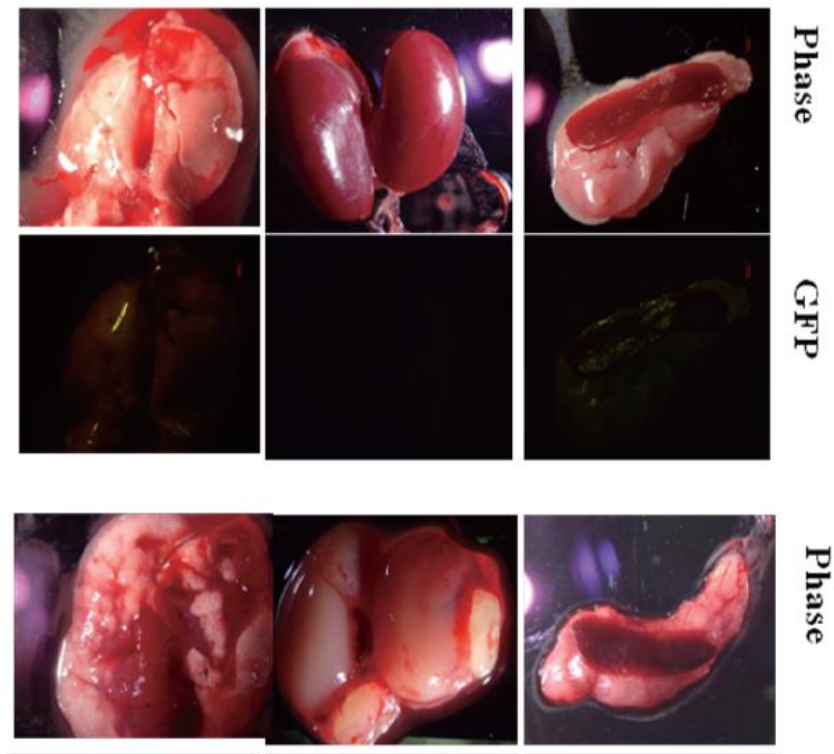

DP

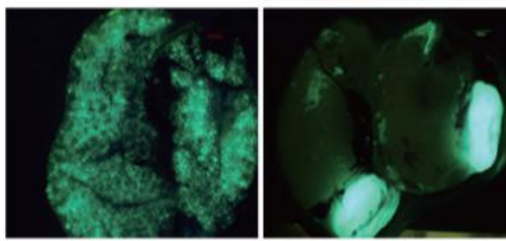

T)

B

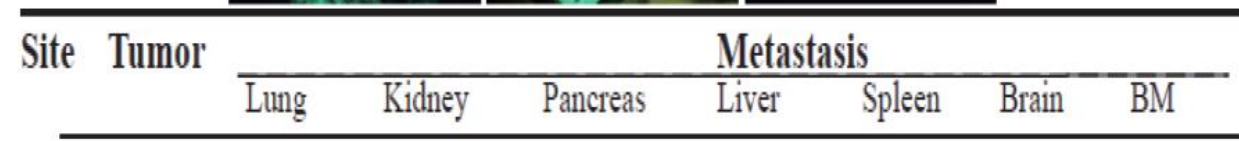

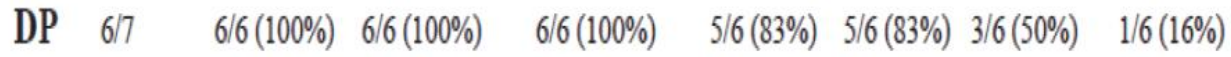

\begin{tabular}{lllllllll} 
S.C & $8 / 8$ & $1 / 4(25 \%)$ & $0 / 4$ & $0 / 4$ & $0 / 4$ & $0 / 4$ & $0 / 4$ & $0 / 4$ \\
\hline
\end{tabular}

C primary tumor lung
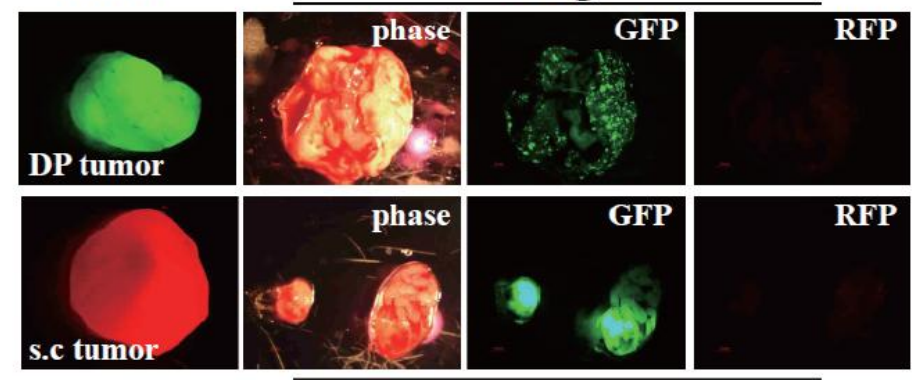

D
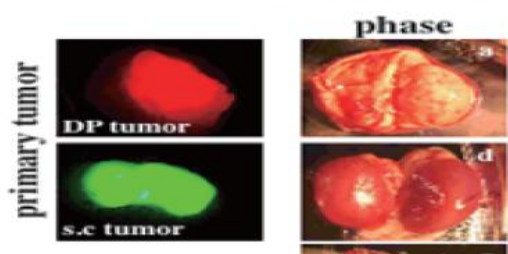

GFP

RFP

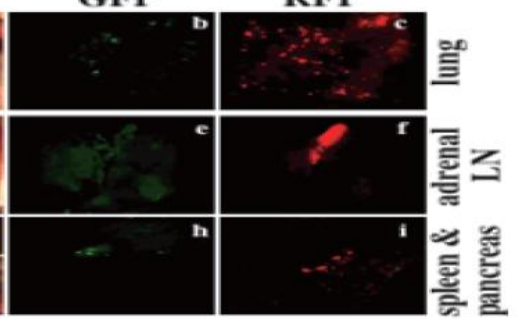




\section{Figure 4.1. Different metastatic potential of PC3 cells implanted in the prostate vs s.c}

(A)10,000 PC3-GFP cells were injected into DP and subcutis, respectively. After 8 weeks, tumors were harvested and metastases were checked in multiple organs. In this figure, whole organ level GFP image showed the metastasis. Lung, kidney, spleen and pancreas were used as the representative metastasis organs. (B)Summary of experiments in A. Tumor incidence and mean tumor weight for each animal were indicated. For DP experiments, 6 of the 7 animals developed tumors. For SC experiments, each animal received two implantations and all 4 animals developed two tumors each. Metastasis was screened by whole organ imaging and incidence was presented (\% in the parentheses). (C) Dual color assays. PC3-GFP and PC3-RFP cells $(100,000$ each) were implanted in the DP and s.c, respectively, in the same mouse, which was terminated 45 days after implantation. Shown are the representative lung and LN images of metastasis. (D)Reciprocal dual color assays. PC3-RFP and PC3-GFP cells (100,000 each) were implanted in the DP and s.c, respectively, in the same mouse, which was terminated 45 days after implantation. Shown are the representative lung and LN images of metastasis. 


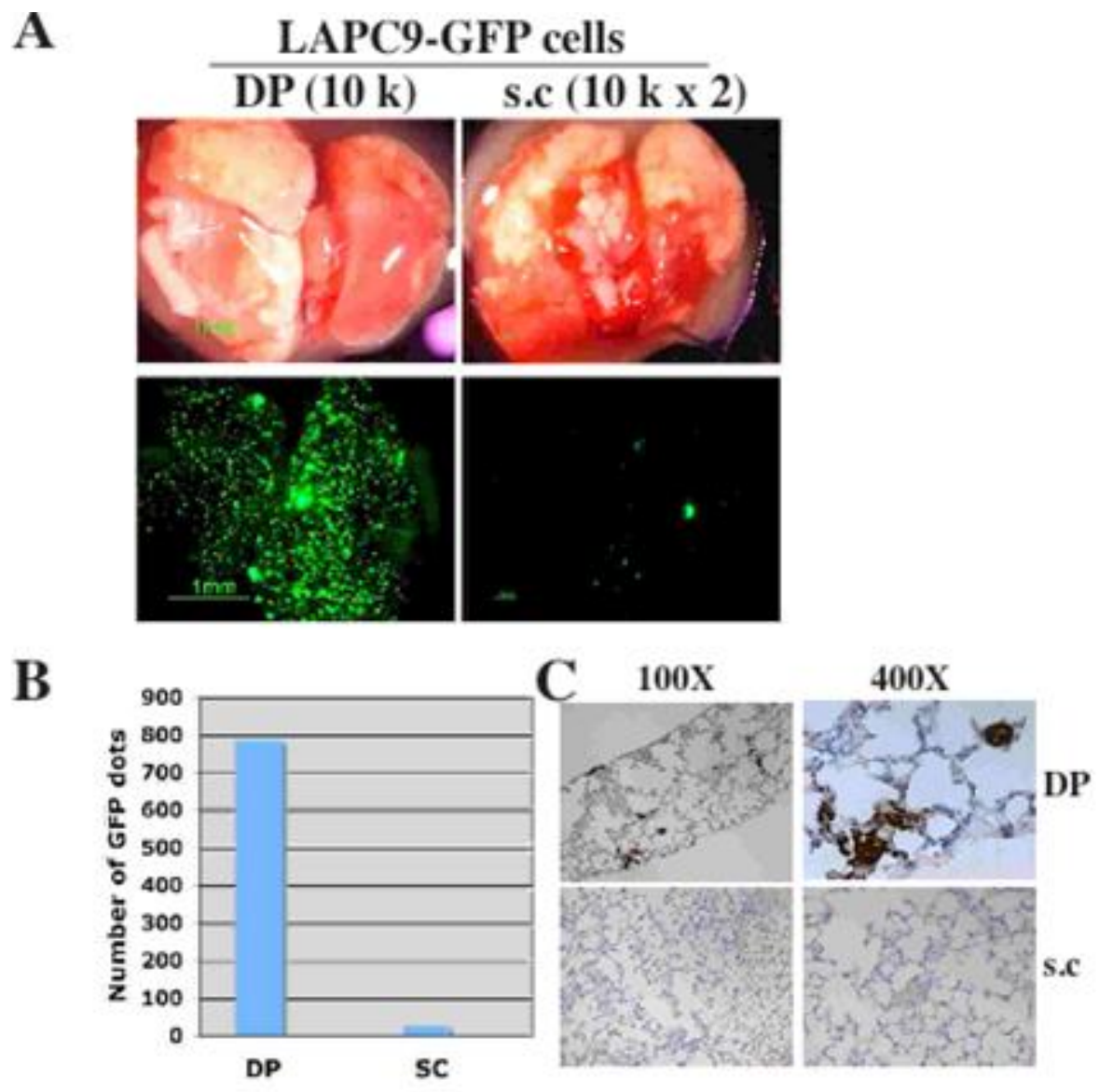

Figure 4.2. Differential Lung metastasis between DP and sc implanted cells $(10,000)$

(A) 10,000 LAPC9-GFP cells were implanted in the DP. Alternatively, two s.c injections each of 10,000 cells were carried out in NOD/SCID mice. All animals were terminated at $\sim 2$ mo. Shown are representative phase and GFP images of the lungs.

(B) Quantification of GFP dots in the lungs shown in (A). (C) Representative IHC GFP staining of lungs from DP and s.c. injected mouse. 
Table 4.1. Tumor-initiating frequency (TIF) of PCa cells subcutaneously (s.c) versus DP implanted in NOD/SCID mice

\begin{tabular}{|c|c|c|c|c|c|c|c|c|}
\hline \multirow[t]{2}{*}{ Cell type } & \multicolumn{6}{|c|}{ Cell dose } & \multirow[t]{2}{*}{ TIF (range) ${ }^{5 s}$} & \multirow[t]{2}{*}{ P value * } \\
\hline & $10^{6}$ & $10^{5}$ & $10^{4}$ & $10^{3}$ & $10^{2}$ & 10 & & \\
\hline \multicolumn{9}{|l|}{ Dul45* } \\
\hline s.c & & $4 / 4$ & $5 / 6$ & $4 / 6$ & $3 / 5$ & & $1 / 3,007(1 / 1,150-1 / 7,864)$ & 0.0016 \\
\hline DP & $3 / 3\left(2 \times 10^{5}\right) 3 / 5\left(5 \times 10^{5}\right)$ & $1 / 4$ & $0 / 4$ & $0 / 4$ & & & $1 / 479,970(1 / 192,816-1 / 1,194,774)$ & \\
\hline \multicolumn{9}{|l|}{$\mathrm{PC}^{*}$} \\
\hline s.c & & $7 / 8$ & $8 / 8$ & $5 / 8$ & $1 / 8$ & $3 / 15$ & $1 / 639(1 / 297-1 / 1,374)$ & 0.00025 \\
\hline DP & $2 / 2\left(5 \times 10^{5}\right)$ & $3 / 3$ & $2 / 4$ & $0 / 3$ & & & $1 / 15,714(1 / 4,211-1 / 58,638)$ & \\
\hline \multicolumn{9}{|l|}{$\mathrm{LNCaP}^{s}$} \\
\hline s.c & & $2 / 4$ & $1 / 4$ & & & & $1 / 106,499(1 / 31,750-1 / 357,234)$ & 0.00015 \\
\hline s.c (with TP) & $2 / 2$ & $3 / 5$ & $2 / 4$ & $0 / 4$ & & & $1 / 69,825(1 / 25,571-1 / 190,664)$ & \\
\hline $\mathrm{DP}$ & $0 / 2\left(2.5 \times 10^{\circ}\right) \quad 0 / 4$ & $0 / 4$ & & & & & & \\
\hline \multicolumn{9}{|l|}{$\mathrm{LAPC}_{4}^{\star *}$} \\
\hline s.c & & & $6 / 6$ & $5 / 8$ & $10 / 12$ & & $1 / 340(1 / 161-1 / 716)$ & 0.00001 \\
\hline $\mathrm{DP}$ & $4 / 5\left(5 \times 10^{5}\right)$ & $1 / 4$ & $0 / 4$ & $0 / 4$ & $0 / 4$ & & $1 / 392,278(1 / 55,640-1 / 2,765,676)$ & \\
\hline \multicolumn{9}{|l|}{$\mathrm{LAPC} 9$ * } \\
\hline s.c & & & $8 / 8$ & $6 / 6$ & $3 / 6$ & & $1 / 142(1 / 47-1 / 428)$ & 0.0004 \\
\hline $\mathrm{DP}$ & $4 / 4$ & $6 / 9$ & $4 / 8$ & $0 / 9$ & $0 / 3$ & & $1 / 58,747(1 / 28,326-1 / 121,841)$ & \\
\hline \multicolumn{9}{|c|}{$\begin{array}{l}\text { "Cultured cells injected in Matrigel (1:1) in female NOD/SCID mice (because both Dul45 and PC3 cells are AR-/PS. } \\
\text { "Cultured cells injected in Matrigel (1:1) with or without testosterone pellet (TP) in male NOD/SCID mice. } \\
\text { "Cells acutely purified from xenograft tumors were injected in Matrigel (1:1) in male NOD/SCID mice. } \\
\text { "Determined by the L-CalcTM software (Stemcell Technologies). The ranges are indicated in the parentheses. } \\
\text { "Determined by..... The fold difference in TIF between s.c versus DP is indicated in parentheses. }\end{array}$} \\
\hline
\end{tabular}

\#Cultured cells injected in Matrigel (1:1) in female NOD/SCID mice (because both Du145 and PC3 cells are AR-(PSA-).

${ }_{\$}$ Cultured cells injected in Matrigel (1:1) with or without testosterone pellet (TP) in male NOD/SCID mice.

\&Cells acutely purified from xenograft tumors were injected in Matrigel (1:1) in male NOD/SCID mice. $\$ \$$ Determined by the L-CalcTM software (Stemcell Technologies). The ranges are indicated in the parentheses.

*Determined by the fold difference in TIF between s.c versus DP is indicated in parentheses. 
A

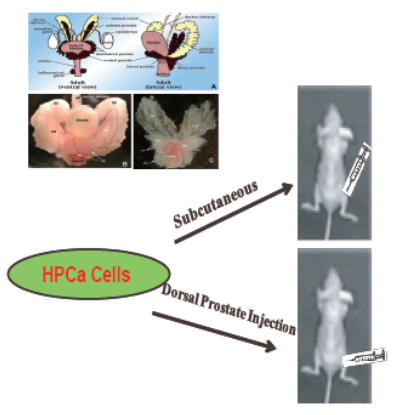

B

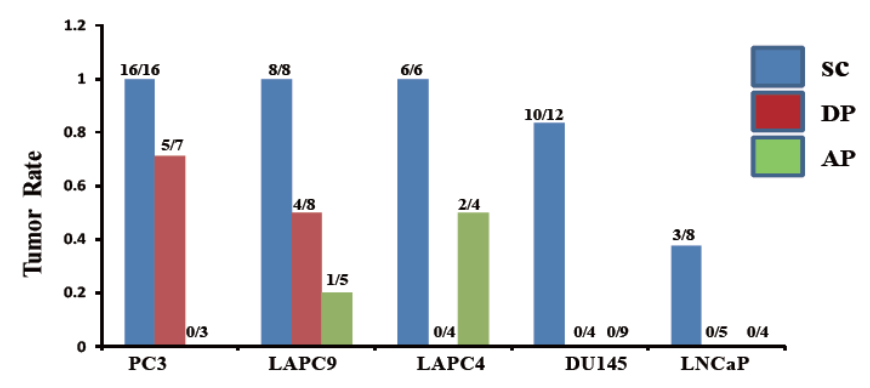

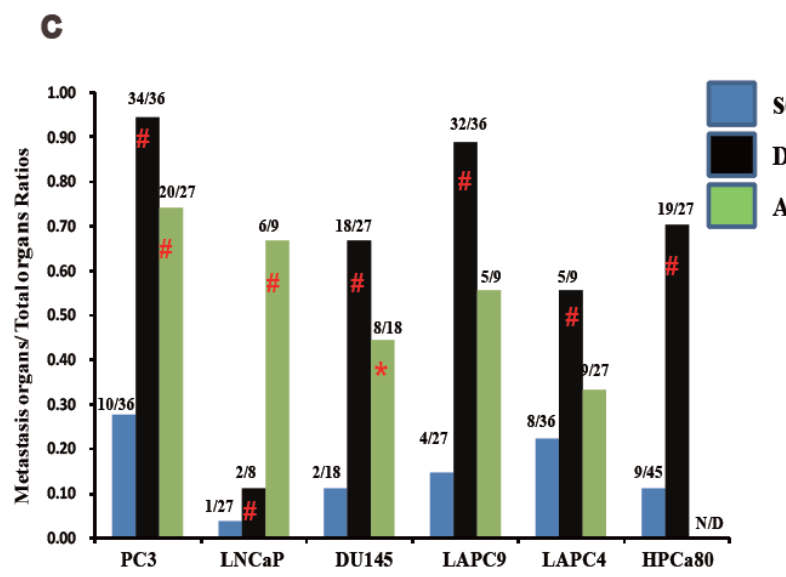

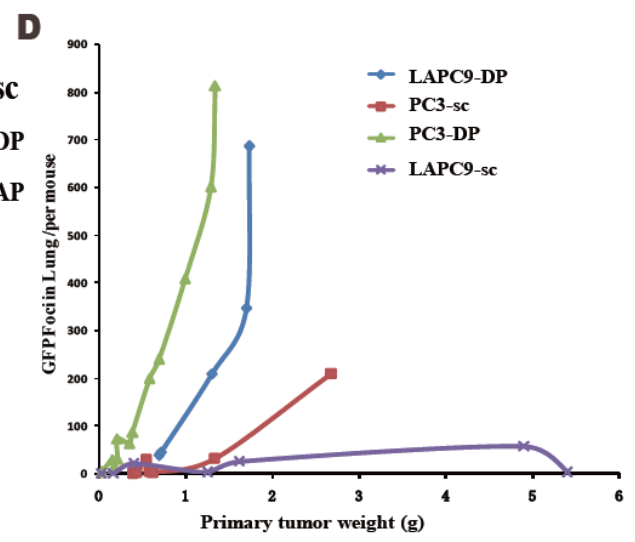

Figure 4.3. Human PCa cells implanted into orthotopic sites demonstrate less primary tumor growth and more widespread metastasis than cells implanted in the ectopic sites

(A) Illustration of mouse DP and AP, as well the DP/AP versus s.c. injection strategy. (B) we implanted 10,000 PC3,DU145, LAPC9, LAPC4 and LNCaP cells each on the three different sites( DP,AP and s.c.), the tumor rate( \# tumors/\# injected mice) were compared and shown in the bar graph. (C) to compare the metastases profiles of different sties implantations, we implanted 100,000 GFP labeled PCa including PC3, DU145, LAPC9,LAPC4, LNCaP and human primary tumor derived lines HPCa80 into two orthotopic sites( DP \& AP) and one ectopic site ( s.c.). When the tumor was harvested, total of nine organs were checked including BM, lung, pancreas, spleen, kidney, liver, brain, adrenal LN, LN( other sites LN except the adrenal LN), GFP dots were checked according the way in (Methods and Materials). We counted the number of GFP invaded organs versus total organs number we checked (organs number $\mathrm{X}$ mice number we checked). The ratio is the organ metastasis rate and used for the bar graph. By compared with s..c model, \# : p $<0.001 * \mathrm{p}<0.05$ (D) a curve graph was made to connect the primary tumor size (tumor weight) with distant lung metastasis (demonstrated by GFP green dots). Each dot of the curve was made by using primary tumor weight labeled in horizontal axis and the responsible lung metastasis in this primary tumor-bearing mouse counted in vertical axis. For each curve, multiple primary tumor-number of lung metastasis dots were applied to form the whole curve. PC3 and LAPC9 PCa lines were injected into DP or s.c. respectively and tumor and lung metastasis were checked. With the same primary tumor weight, the lung metastasis is dramatically different from different transplant site tumors. 
In table 4.1, we found a very surprise phenomenon, when these identical PCa cells were put into two different transplant sites, they exhibited dramatically different tumorigenesis potential. Subcutis implantation improved tumor growth with the presence of significant higher Tumorinitiating frequency (TIF). Additionally, because AP is another orthotopic site for PCa, we further test the tumor grow and metastasis profiles in AP. When you injected 10,000 cells of each PCa lines into these three sites( DP, AP, and s.c.), as shown in figure $4.3 \mathrm{~B}$, the tumor rates are also significant different between s.c. site and two orthotopic sites. The DP site seems to be less conducive for tumor growth, as 10,000 cells could lead to tumors only in PC3 and LAPC9, two of the most aggressive prostate cancer lines, but not in in LAPC4, DU145 and LNCaP. In contrast, the sc site more easily allows prostate cancer line to produce tumors. Either DP and AP has much lower tumor rates compared with s.c. sites. These results revealed that these two sites are different in promoting tumor growth.

Since our main purpose is to analyze metastasis and we already showed that PC3 and LAPC9 have more DP derived metastasis (Figure 4.1, 4.2), we further test the metastasis potential of AP model. We expected to demonstrate that not only the specific DP site, but the orthotopic environment promotes metastases. By using GFP label cells, we found not only DP models show enhanced metastasis production, but AP model exhibit the same phenomenon as well ( figure 4.4). AP-implanted PC3 cells, much like DP-injected cells, showed extensive dissemination (Figure 4.4).

Metastasis usually is not limited in one single organ. Hence, we did a comprehensive check of metastasis in multiple organs including lung, spleen, pancreas, liver, kidney, renal lymph node, brain and bone marrow. In order to quantify these metastasis profiles between mice, we 
A $\underset{\text { (Duit45 } 1 \text { mil; AP) }}{\operatorname{animal} \# 826}$ B (Du145 1 mil; AP)

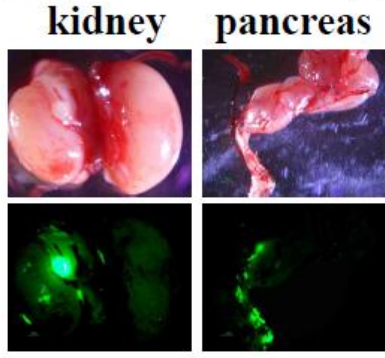

C animal $\# 572$ \begin{tabular}{lll} 
(LNCaP 1 & mil; AP) \\
\hline lung kidney
\end{tabular}

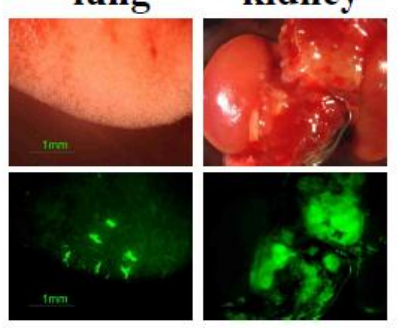

D

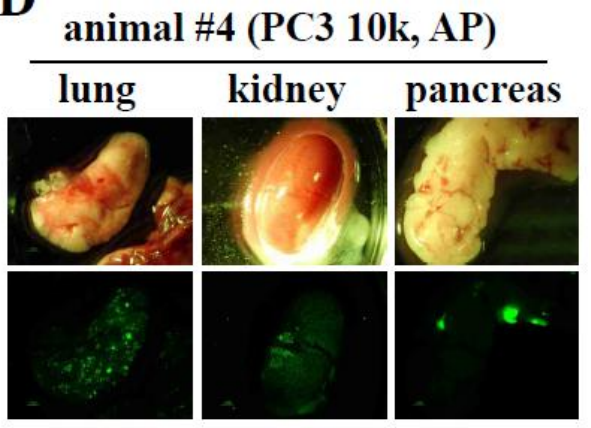

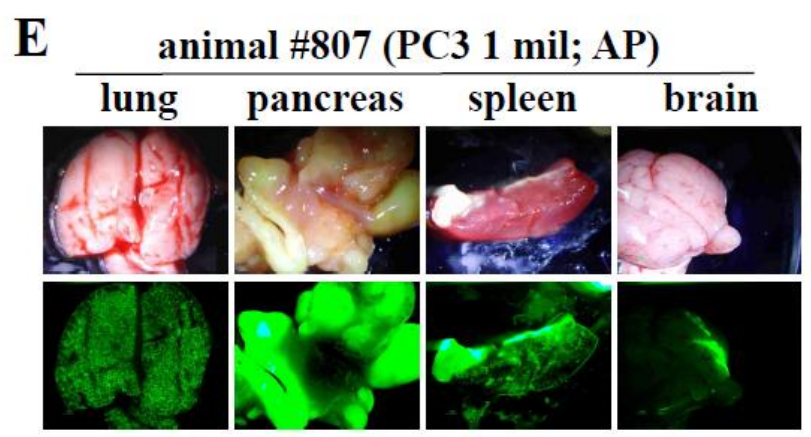

Figure 4.4. The AP-implanted PCa-GFP cells metastasize to multiple organs

Shown are representative organ images (upper panels, phase; lower panels, GFP) of 1 million Du145-GFP cells (A and B), 1 million LNCaP-GFP cells (C) and 10,000 (D) or 1 million (E) of PC3-GFP cells implanted in the AP of NOD/SCID mice and harvested $\sim 60$ days post implantation. Animal numbers are indicated on top. 

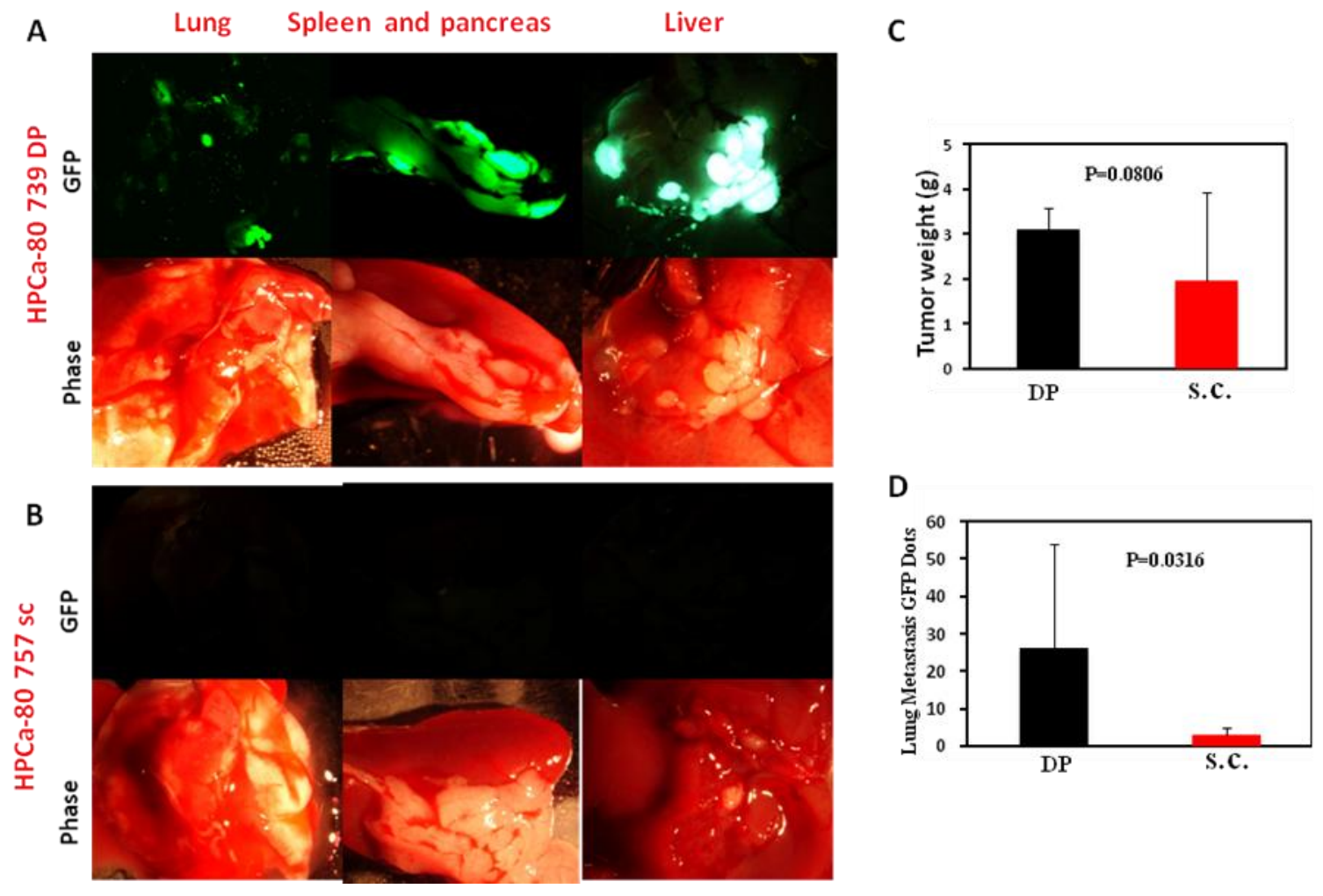

Figure 4.5 HPCa-80 Tumor growth and metastasis profiles in two implantation site DP vs sc

We developed HPCa80 cells from human primary patient and maintained in mice in vivo. After dissociated the tumor and these single HPCa80 cells were infected with our GFP vector. Then we implanted 500,000 HPCa-80-GFP cells into DP and s.c. respectively. (A) GFP and phase image of mouse lung, spleen, pancreas, and liver from HPCa80 DP implantation (500,000 cells) are exhibited. GFP dots in organ showed metastasis. (B) GFP and phase image of lung, spleen, pancreas, and liver from HPCa80 s.c. implantation (500,000 cells). (C) Tumor weight of DP and s.c. tumor, no significance was found. (D) Lung metastasis GFP dots were counted in both DP and sc tumor lung and has significantly difference between these two models. 
designed an index called "Metastatic ratios", which are calculated by dividing the number of metastasis-invaded organs by the total number of organs (Figure 4.3-C). Moreover, we applied a human primary tumor derived line HPCa80 to test the DP and s.c. site in tumor growth and metastasis (figure 4.5). Consistently, HPCa80 in DP also produce more metastasis. By calculating the metastatic ratio, we clearly indicate that DP tumors is superior in developing more widespread metastasis than sc tumors in all of these lines despite the higher tumor rate seen in sc environments. On the other hand, we already know that primary tumor size is associated to tumor metastasis. The bigger the primary tumor, the more metastases disseminate. It would miss some critical points for metastasis potential if we only compare with metastasis regardless of primary tumor weight. It would be more accurate to compare metastasis potential by using the same size primary tumor. Nevertheless, it may be less efficiency to compare multiple organs as the index for metastasis potential. Then we chose lung as the representative organ since it is one of the most frequent sites for PCa cell metastasis in mouse models (An et al., 1998). Even one of the weakest metastatic cancer cell lines, LNCaP, produced lung metastasis after four-week incubation (Zhou et al., 2002). We estimated lung metastasis by counting the GFP dots in each image. In order to better appreciate the metastatic potential of different primary tumors, we collected a series of data of primary tumor sizes and their lung metastases from DP and sc tumors originally derived from two different prostate cancer lines, PC3 and LAPC9, and developed a tumor size-lung metastasis correlated curve (In Figure 4.3D). In the tumor size-lung metastasis curve which we developed, we first confirmed the positive correlation between lung metastasis and tumor size: The bigger the tumor, the more lung metastasis observed. Looking at DP or sc tumors alone in either PC3 or LAPC9 lines, the metastasis increases with increasing 
size of the primary tumor. Second, we found that tumor weight-standardized metastasis increases with tumor aggressiveness in both DP and sc models. For example, PC3 is more aggressive than LAPC9; a one-gram PC3 tumor has a standardized lung metastasis of around 500, while the value for an LAPC9 DP metastasis is around 160. Significantly, DP tumors develop much more lung metastasis than sc tumors of the same primary tumor size. A one-gram PC3 DP tumor lung metastasis value is around 500, while the sc tumor has a value of only 30 (dots), underscoring a striking 16-fold difference between DP and sc. Moreover, for even the four gram sc tumor, the lung metastasis value is only around 200, still less than half that of the one-gram DP tumor. The same trend was also observed in LAPC9 tumors as seen in Figure 4.3D.

Individual differences between mice may influence metastasis progression, so, in order to remove this individual difference, we developed a dual-color metastasis assay, in which we injected PC3-RFP cells s.c and implanted the same number of PC3-GFP cells in the DP of the same recipient mouse. As shown in Figure 4.1.C-D, we observed much more GFP foci than RFP foci in the lung and lymph nodes. Reciprocating the color by injecting PC3-GFP cells s.c and implanting PC3-RFP cells in the DP revealed significantly more RFP foci in the lung and lymph node(Figure 4.1D).

In the DP model, the question of metastasis by fluid leakage during injection arose. To exclude any possibility that the $\mathrm{GFP}^{+}$human PCa cells observed in mouse organs resulted from injection-related leakage of tumor cells directly into the blood stream, we performed a time course study by implanting PC3-GFP cells into the DP and sacrificing mice at one to seven 
Table 4.2. Time course of DP-injected PC3-GFP cells

\begin{tabular}{|c|c|c|c|c|c|c|c|c|c|c|}
\hline \multirow{2}{*}{$\begin{array}{l}\text { Time }^{a} \\
\text { (after injection) }\end{array}$} & \multirow{2}{*}{$\begin{array}{l}\text { Termination time } \\
\text { (Inj. \& term. dates) }\end{array}$} & \multicolumn{9}{|c|}{ Metastasis $^{c}$} \\
\hline & & Femur & Tibia & Lung & Kidney & Liver & Pancreas & Brain & Spleen & $\mathrm{LN}^{\mathrm{d}}$ \\
\hline PC3-GFP $1,500,000$ (DP) & Inj:5/19/06 & & & & & & & & & \\
\hline \multicolumn{11}{|l|}{1 week } \\
\hline 686 (animal tag) & term:5/26/06 & $\cdot$ & - & $\cdot$ & - & $\cdot$ & - & $\cdot$ & $\cdot$ & - \\
\hline 679 & term:5/26/06 & - & - & - & - & - & - & - & - & - \\
\hline 678 & term:5/26/06 & - & - & - & - & - & - & - & - & - \\
\hline \multicolumn{11}{|l|}{2 weeks } \\
\hline 682 & term: $6 / 02 / 06$ & - & - & \pm & - & - & \pm & - & - & - \\
\hline 683 & term: $6 / 02 / 06$ & - & - & \pm & - & - & \pm & - & - & - \\
\hline 684 & term: $6 / 02 / 06$ & - & - & \pm & - & - & \pm & - & - & - \\
\hline 685 & term: $6 / 02 / 06$ & - & - & - & - & - & \pm & - & - & - \\
\hline \multicolumn{11}{|l|}{3 weeks } \\
\hline \#1 (animal tag) & term: $6 / 09 / 06$ & $\cdot$ & $\cdot$ & \pm & + & + & ++ & $\cdot$ & ++ & + \\
\hline$\# 2$ & term: $6 / 09 / 06$ & - & $\cdot$ & \pm & - & $\cdot$ & $\cdot$ & $\cdot$ & $\cdot$ & - \\
\hline$\# 3$ & term: $6 / 09 / 06$ & - & - & + & $\cdot$ & + & \pm & - & $\cdot$ & + \\
\hline$\# 4$ & term: $6 / 09 / 06$ & - & - & \pm & - & - & - & - & - & - \\
\hline \multicolumn{11}{|l|}{4 weeks } \\
\hline$\# 1$ & term:6/16/06 & - & - & + & - & $\cdot$ & + & - & - & ++ \\
\hline$\# 2$ & term: $6 / 16 / 06$ & - & - & + & + & + & ++ & - & + & ++ \\
\hline$\# 3$ & term: $6 / 16 / 06$ & - & - & + & + & + & + & - & + & + \\
\hline \multirow{2}{*}{\multicolumn{11}{|c|}{5 weeks }} \\
\hline & & & & & & & & & & \\
\hline$\# 1$ & term: $6 / 23 / 06$ & - & - & ++ & ++ & \pm & ++ & - & + & +++ \\
\hline$\# 2$ & term:6/23/06 & - & - & ++ & ++ & \pm & ++ & - & + & ++ \\
\hline \multicolumn{11}{|l|}{6 weeks } \\
\hline \#1 & term: $6 / 30 / 06$ & - & $\cdot$ & +++ & +++ & + & ++ & - & + & +++ \\
\hline$\# 2$ & term:6/30/06 & - & - & ++ & ++ & \pm & + & - & + & ++ \\
\hline$\# 3$ & term:6/30/06 & - & - & ++ & ++ & + & ++ & - & + & ++ \\
\hline$\# 4$ & term:6/30/06 & - & $\cdot$ & ++ & ++ & \pm & ++ & - & + & ++ \\
\hline$\# 5$ & term:6/30/06 & - & - & +++ & +++ & + & ++ & - & + & +++ \\
\hline \multicolumn{11}{|l|}{7 weeks } \\
\hline$\# 1$ & term:7/07/06 & \pm & $\cdot$ & +++ & +++ & + & ++ & \pm & + & +++ \\
\hline$\# 2$ & term:7/07/06 & \pm & - & +++ & +++ & + & ++ & \pm & + & +++ \\
\hline$\# 3$ & term:7/07/06 & - & - & +++ & +++ & + & ++ & + & + & +++ \\
\hline$\# 4$ & term:7/07/06 & - & - & +++ & ++ & + & ++ & $\cdot$ & ++ & ++ \\
\hline \#5 & term:7/07/06 & - & - & +++ & ++ & \pm & + & - & + & ++ \\
\hline
\end{tabular}


weeks after injection. We observed that there were no $\mathrm{GFP}^{+}$cells in blood (not shown) nor in any organ one week post surgery (Table 4.2). By the second week, 1-5 GFP ${ }^{+}$PCa cells began to appear in the lung and pancreas and by the third to fourth week, low levels of $\mathrm{GFP}^{+}$PCa cells were detected in most organs examined, which further increased thereafter (Table 4.2). By the seventh week, sporadic GFP ${ }^{+}$PC3 cells were observed in the femur (Table 4.2).

All together, we did a very comprehensive study on metastasis and tumor growth pertaining to these two sites. We not only evaluated the metastatic rate, but also quantified the lung metastasis and used this as the major index to reflect the overall metastatic potential. We further linked metastasis with primary tumor weight, showing more clearly that the high metastatic potential of DP or AP is not due to the primary tumor size. When these cancer cells are put in different sites, they are initially identical. Hence, this metastatic difference might be mainly due to microenvironmental modifications (or transplantation site). On the other hand, sc tumors with the same size as in the orthotopic sites develop much less metastasis. Meanwhile sc tumors also have a higher tumor-initiating rate than DP tumors. This significant separation of metastasis and tumor growth suggests that the tumor-initiating cells and metastatic cells need not always be the same cell population. Since the sc model is widely used as a CSC study model, it implies that CSCs may contain two subpopulations: One is the tumor-initiating CSC, the other is a metastatic CSC. There may be more/enriched metastatic CSC in the mouse DP model while sc tumors mostly contain tumor-initiating cells. On the other hand, it also hint that there might be a real CSC subpopulation that can both initiate tumor and develop metastasis in DP tumors, while sc tumors may contain more progenitor cells which have a limited ability to produce metastasis. 
Gene Expression Reveal that Not Only Invasive/metastatic/inflammatory Genes Respond to the Highly Metastatic Niche in Mouse DP, but also Development Pathway and Stem Cell-Related Genes Are Up-regulated in DP Tumors.

Gene microarrays are widely used in exploring the molecular mechanisms operating in tumor microenvironments and metastasis. However, there are still only a few studies focusing on the unique mechanism of orthotopic microenvironment-induced metastasis. Most current tumor microenvironment microarray analyses mainly use a single component of the tumor microenvironment, such as fibroblast cells, or a micro-dissected part as representative of the whole tumor microenvironment. In addition, these studies have used in vitro cultured cancer cell lines but not in vivo tumor samples as the experimental model (Nakamura et al., 2007). Additionally, many of these studies usually only focus on one or a few specific pathways that are related to interaction between tumor microenvironment and metastasis (Fidler et al., 2007). This may not give the entire picture of the interaction between the tumor and its microenvironment. By performing microarray on tumor samples implanted into the pancreas and subcutis, Nakamura et al provided solid evidence to show a significantly different gene expression pattern was evident in these two kinds of tumors (Nakamura et al., 2007). Their study suggests that these tumor microenvironments influence primary gene expression. However, this group failed to establish a clear mechanism "map" to show what genes are involved in regulating metastasis. One of the explanations as to why they are unable to discover a clear gene signature may be due to the individual difference between mice in the gene microarray platform. We showed previously that DP and sc tumors which develop in same mouse exhibited dramatic metastatic difference in our dual color label tumor system. 
Hence, we designed the microarray to analyze samples from the same mouse in order to eliminate between-mouse variability.

We injected 10,000 PC3-GFP cells s.c and into the DP of the same male NOD/SCID mouse. Three pairs of DP and s.c-grown PC3 tumors were harvested from each of three mice (\#714,717 and 718) and total RNA was isolated for microarray experiments using the Agilent "Human/mouse Whole Genome Oligo Microarray (44K) Kit"( Figure 4.6). Since the isolated tumor RNA contains both a human cancer cell species and a host stromal species, we carried out both human and mouse array analyses using the same sample. In the human array, we only chose those genes which were commonly upregulated /downregulated two-fold or greater. Since these up-regulated genes may be some of the genes related to metastasis, we found 593 genes were commonly overexpressed in DP tumors in all three arrays (Figure 4.6). Not only these three pairs of tumor show the same commonly up-down regulated genes, but also the purified human epithelial cancer cells array demonstrate the same trend (figure4.6). This further confirmed the accurate of our microarray. In sharp contrast, most genes down-regulated in the DP tumors remained unclear. We did not identify metastasis/inflammation/stem cell related genes in the list of down-regulated genes. By gene ontology analysis, as shown in Figure $4.7 \mathrm{D}$ and table 4.3 , as we expected, $12.5 \%$ of the identified genes are related to migration/ invasion/metastasis; These genes include OPN, MMP9, MMP10, IGFBP5, SPARC, ADAM family and ESM1. 7.5\% of them are inflammation-related genes, chemokines, and cytokines, including IL-1 $\beta$, CXCR4/CXCL12, CCR1, and TLR-6. It is already well-known that inflammation contributes to the highly metastatic microenvironment and promotes 


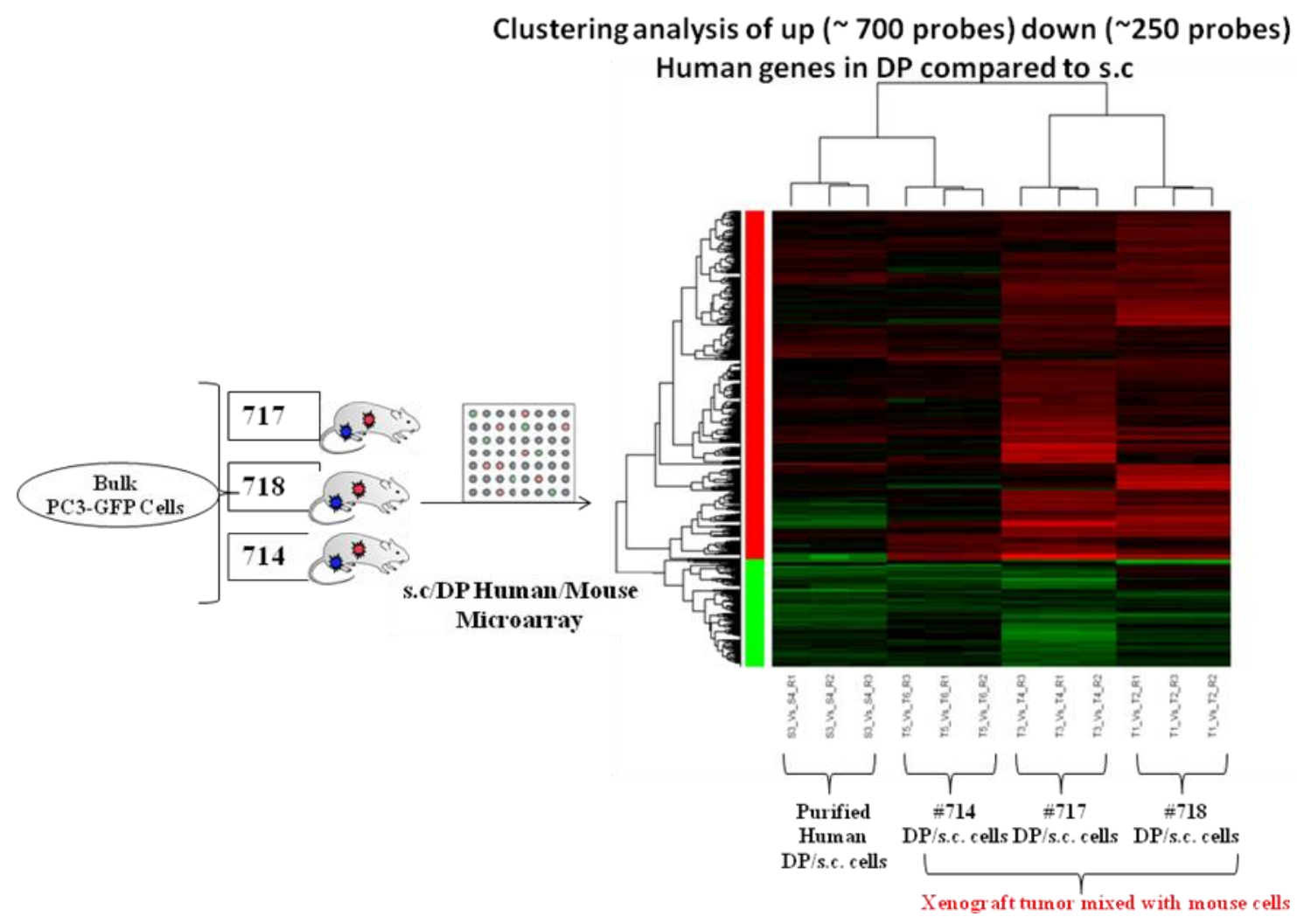

Figure 4.6. Schematic chart for gene microarray and clustering analysis up-regulated human genes

100,000 PC3-GFP cells were injected into DP and s.c. respectively in same mouse (717,718,714). DP and s.c. tumors were harvested after 8 weeks, the RNA were isolated to perform human and mouse genomic microarray (see methods and material section). For each mouse, we perform triplicate arrays; hence we totally have nine pairs of arrays). We also purified human epithelial cancer cells from xenograft tumor and perform the same microarray. By clustering analysis, both purified human cells and xenograft cells showed the same trend in microarray. 


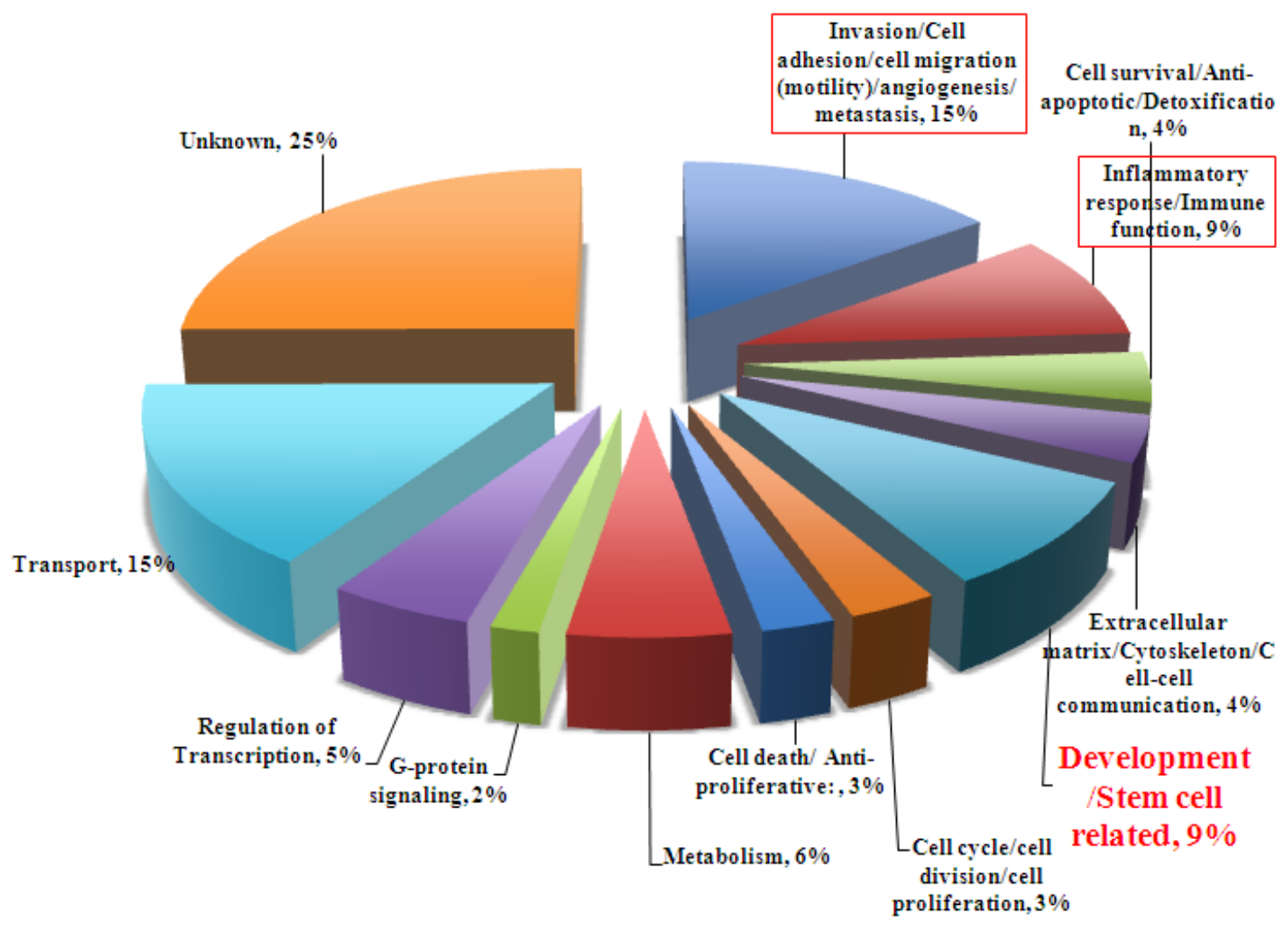

Figure 4.7 Pie graph showed the Gene Ontology analysis of DP up-regulated human genes 
Table 4.3. Representative human genes commonly upregulated in DP versus s.c tumors

\begin{tabular}{|c|c|c|c|c|}
\hline Gene name & Systematic name & Description & Fold increase* & P value $^{\#}$ \\
\hline \multicolumn{5}{|c|}{ Proteases/invasion/angiogenesis } \\
\hline SPP1 & NM000582 & secreted phosphoprotein 1 (osteopontin) & $9.4-11.0(10 \mathrm{x})$ & $0.02-0.03$ \\
\hline IGFBP5 & NM000599 & insulin-like growth factor binding protein 5 & $2.2-4.9(3 x)$ & $0.03-0.04$ \\
\hline HGF & NM001010931 & hepatocyte growth factor (scatter factor) & $2.1-4.5(10 \mathrm{x})$ & $0.04-0.007$ \\
\hline SPARC & NM003138 & secreted protein, acidic, cysteine-rich (osteonectin) & 2.2 & $1.2 \times 10^{-6}$ \\
\hline MMP9 & NM004994 & $\begin{array}{l}\text { matrix metallopeptidase } 9 \text { (gelatinase } \mathrm{B} \\
92 \mathrm{kDa} \text { gelatinase, } 92 \mathrm{kDa} \text { type IV collagenase) }\end{array}$ & $5.5-5.9(10 \mathrm{x})$ & all $\sim 0.03$ \\
\hline MMP10 & NM002425 & matrix metalloproteinase 10 (stromelysin 2 ) & $4.8-1.9-10$ & 0.001 \\
\hline MMP1 & NM002421 & matrix metallopeptidase 1 (interstitial collagenase) & $2.13-2.18(10 \mathrm{x})$ & all $\sim 0.001$ \\
\hline ADAMTS6 & NM197941 & ADAM metallopeptidase with TSP type 1 motif, 6 & 4.6 & 0.004 \\
\hline ADAM23 & NM003812 & ADAM metallopeptidase domain 23 & 3.7 & 0.004 \\
\hline ADAM22 & NM021721 & ADAM metallopeptidase domain 22 & $2.3,2.7$ & $<10^{-5}$ \\
\hline ESM1 & NM007636 & endothelial cell-specific molecule 1 & $6.3,11.3$ & both $<10^{-5}$ \\
\hline AGTR1 & NM031850 & angiotensin $\Pi$ receptor, type 1 & 4.2 & $4.4 \times 1^{-6}$ \\
\hline AGT & NM000029 & angiotensinogen (serpin peptidase inhibitor, clade $\mathrm{A}$ ) & $2.0-2.4(9 x)$ & $<0.0001$ \\
\hline HIF3A & AK024095 & weakly similar to HIF-1 $\alpha$ & 2.1 & $4 \times 10^{-6}$ \\
\hline EDNRA & NM001957 & endothelin receptor type $A$ & 2.0 & 0.006 \\
\hline \multicolumn{5}{|c|}{ Inflammation/cytokines } \\
\hline $\mathbb{L}-1 \beta$ & NM000576 & interleukin-1 $\beta$ & $4.36-4.4(10 \mathrm{x})$ & all $\sim 0.01$ \\
\hline IL1R1 & NM000877 & interleukin 1 receptor, type I & 2.6 & 0.005 \\
\hline IL13RA2 & NM000640 & interleukin 13 receptor, alpha 2 & 2.7 & 0.005 \\
\hline CXCL7 & NM002704 & pro-platelet basic protein (PPBP) & 5.0 & 0.005 \\
\hline CXCR4 & NM001008540 & chemokine (C-X-C motif) receptor 4 & $4.7-5.0(10 \mathrm{x})$ & $<0.0005$ \\
\hline CXCL5 & NM002994 & chemokine (C-X-C motif) ligand 5 & $2.2,3.5$ & 0.04 \\
\hline CCR1 & NM001295 & chemokine (C-C motif) receptor 1 & 2.7 & 0.002 \\
\hline CCR3 & NM001837 & chemokine (C-C motif) receptor 1 & 2.1 & 0.002 \\
\hline CXCL12 & NM199168 & chemokine (C-X-C motif) ligand 12 (SDF-1) & 2.0 & 0.0003 \\
\hline LCP1 & NM002298 & lymphocyte cytosolic protein 1 (L-plastin) & 4.1 & 0.01 \\
\hline PLD5 & NM152666 & phospholipase D family, member 5 & 3.2 & 0.0003 \\
\hline TNFS15 & NM005118 & tumor necrosis factor (ligand) superfamily, member 15 & 3.0 & 0.01 \\
\hline ALOX5AP & NM001629 & arachidonate 5-lipoxygenase-activating protein & 3.0 & 0.004 \\
\hline NOS2A & NM000625 & nitric oxide synthase $2 \mathrm{~A}$ (inducible) & $2.7-3.8(10 \mathrm{x})$ & $0.01-0.009$ \\
\hline PLCG2 & NM002661 & phospholipase $\mathrm{C}$, gamma 2 & 2.7 & 0.015 \\
\hline TLR6 & NM006068 & toll-like receptor 6 & 2.1 & 0.0003 \\
\hline C3AR1 & NM004054 & complement component 3 a receptor 1 & 2.5 & $7.6 \times 10^{-5}$ \\
\hline CF1 & NM000204 & complement factor I & 2.5 & 0.003 \\
\hline CFP & NM002621 & complement factor properdin & 2.1 & 0.0004 \\
\hline RGC32 & NM014059 & response gene to complement 32 & $2.0,2.1$ & 0.002 \\
\hline C1QTNF2 & NM031908 & $\mathrm{Clq}$ and tumor necrosis factor related protein 2 & 2.0 & 0.002 \\
\hline \multicolumn{5}{|c|}{ Developmental pathways/stem cell-related } \\
\hline PROM1 & NM006017 & prominin 1 (CD133); a stem cell marker & 3.3 & 0.007 \\
\hline FGF7 & NM002009 & fibroblast growth factor 7 (keratinocyte growth factor) & 3.3 & $3.7 \times 10^{-7}$ \\
\hline $\mathrm{ABCG} 2$ & NM004827 & ATP-binding cassette, sub-family $\mathrm{G}$ & 2.7 & 0.01 \\
\hline $\mathrm{CD} 24$ & L33930 & a CSC marker, related to $\mathrm{PCa}$ metastasis & 2.5 & 0.045 \\
\hline KITLG & NM000899 & KIT ligand & 2.5 & 0.0008 \\
\hline HOXD13 & NM000523 & homeobox D13 & 2.5 & 0.008 \\
\hline ID3 & NM002167 & inhibitor of DNA binding 3, dominant negative $\mathrm{HLH}$ protein & 2.3 & 0.001 \\
\hline NKX3.1 & NM006167 & NK3 transcription factor related, locus 1 & 2.3 & $2.4 \times 10^{-5}$ \\
\hline WNT4 & NM030761 & wingless-type family, member 4 & $2.2,2.3$ & $0.01,0.02$ \\
\hline FOXJ1 & NM001454 & forkhead box J1 & 2.0 & 0.017 \\
\hline HOXB9 & NM024017 & homeobox B9 & 2.0 & 0.03 \\
\hline TGFBI & NM000358 & transforming growth factor, beta-induced, $68 \mathrm{kDa}$ & 4.2 & 0.048 \\
\hline BMPR1B & NM001203 & bone morphogenetic protein receptor, type IB & 3.3 & $2.1 \times 10^{-6}$ \\
\hline BAMBI & NM12342 & BMP and activin membrane-bound inhibitor & 2.4 & 0.047 \\
\hline INHBA & NM002192 & inhibin, beta $\mathrm{A}$ (activin A) & 2.4 & 0.001 \\
\hline CHRD & NM003741 & chordin & 2.4 & 0.01 \\
\hline TGFB2 & NM003238 & transforming growth factor, beta 2 & 2.2 & 0.04 \\
\hline SMAD1 & NM005900 & SMAD, mothers against DPP homolog 1 & 2.1 & 0.0002 \\
\hline BMP3 & NM001201 & bone morphogenetic protein 3 (osteogenic) & 2.0 & 0.002 \\
\hline
\end{tabular}


metastasis (Mantovani et al., 2008). Both metastatic and inflammatory genes' presence show that not only is our microarray accurately associated with highly metastatic DP tumors, but also further confirmed that this high metastatic potential is truly linked to the tumor microenvironment. Most importantly, a cluster of developmental and stem cell-related genes (7.8\%) is highly up-regulated in DP tumors. These well- known stem cell markers such as CD133, ABCG2, CD24, WNT4, HOXB9, and NKX3.1, were all reported as CSC markers or CSC-related genes recently (Li et al., 2007a; O'Brien et al., 2007; van den Berg et al.; Wang et al., 2009). This CSC signature might be the most significant point of the microarray. It provides evidence that the primary tumor microenvironment may promote metastasis through regulation of a CSC phenotype. Linking metastasis with CSCs is not new; recently Weinberg's group's work and Glinsky's work both showed that stem cell signatures were discovered from malignant metastatic patient samples (Ben-Porath et al., 2008; Glinsky et al., 2005). This is not surprising since the first solid CSC phenotype was also derived from clinical metastatic samples (Al-Hajj et al., 2003).

However, the biggest difference between our studies and previous studies is that our study mainly focuses on primary tumors microenvironment. It clearly indicates that these CSClinked metastatic properties may not only present in those distant metastatic cells, but may also exist in primary tumors. It may be the first time to report this primary tumor microenvironment enriched with CSC features. It is very possible that those distant metastatic cells attain CSC properties before they leave the primary tumor. It also implies that tumor primary microenvironment is equally critical as the secondary metastatic microenvironment. On the 
A

\begin{tabular}{llll}
\hline Gene name & \multicolumn{3}{c}{ DP tumors /fold changes } \\
& animal 714 & animal 717 & animal 718 \\
\hline SPP1 (osteopontin) & 4352.4 & 775.2 & 15.1 \\
IGFBP-5 & 41.9 & 65.4 & 1.6 \\
MMP9 & 813.5 & 38.6 & 4.8 \\
ESM1 & 42.2 & 6644.5 & 3.9 \\
IL-1 $\beta$ & 83.3 & 125.7 & 1.6 \\
CXCR4 & 3.0 & 198 & 1.2 \\
TGF- $\beta$ 1 & 1.9 & 1.6 & 1.3 \\
TGF- $\beta$ 2 & 2.3 & 10.5 & 0.7 \\
Bcl-2 & 5.2 & 23.8 & 1.3 \\
& & & \\
TNFSF10 & & & 0.3 \\
SFRP1 & 0.2 & 0.3 & 0.4 \\
\hline
\end{tabular}

B

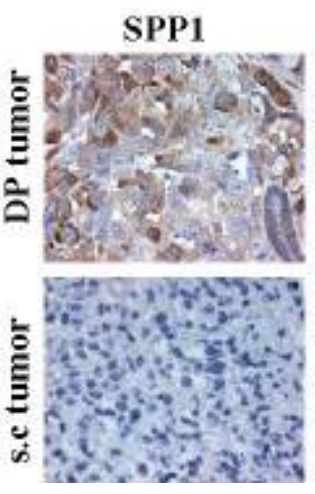

MMP9

ESM1
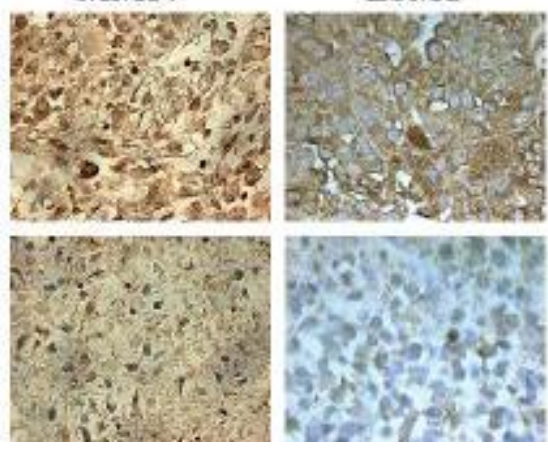

CXCR4
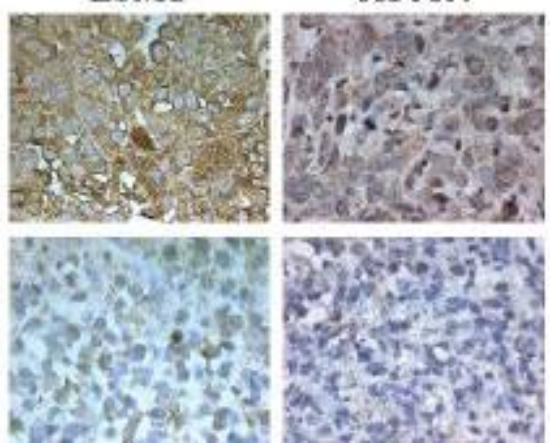

Figure 4.8. Verification of gene expression identified by microarray

(A) qRT-PCR confirmation of mRNA changes of representative genes in PC3 tumors implanted in the DP of NOD/SCID mice, Nine genes commonly upregulated (see Table 4.1) and two genes commonly downregulated (TNFSF10 and SFRP1) identified in the human microarray analysis were selected for independent confirmation using quantitative real-time RT-PCR. Values greater than 1 indicate upregulation in the DP tumors and values lower than 1 indicate downregulation in the DP tumors compared to the corresponding s.c PC3 tumors in the same host animal. qRT-PCR table (B) Several commonly up-regulated genes selected from A including SPP1, MMP9, ESM1 and CXCR4 were validated by IHC in PC3 tumors implanted in the DP of NOD/SCID mice. 
A

755 s.c. $759 \mathrm{DP} \quad 761$ s.c. $759 \mathrm{DP} \quad 261$ s.c. $261 \mathrm{DP}$

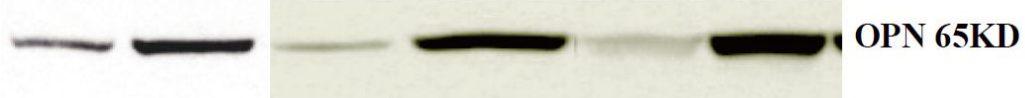

$\longrightarrow \longrightarrow$ B-Actin

B

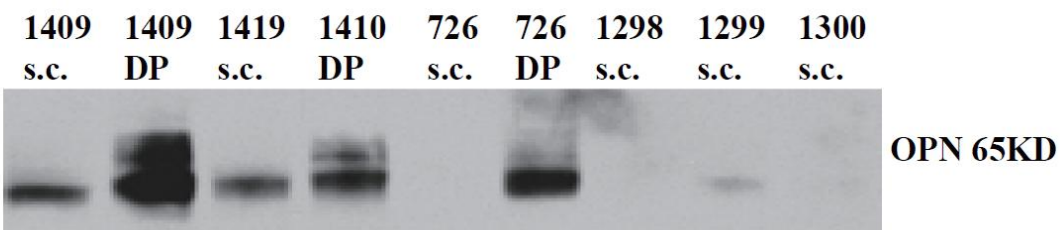

$\mathbf{C}$

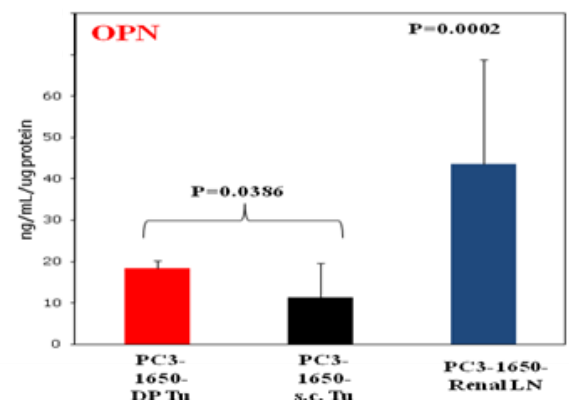

D

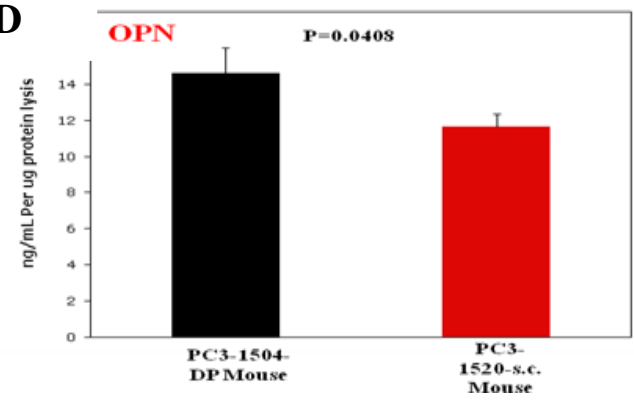

$\mathbf{E}$

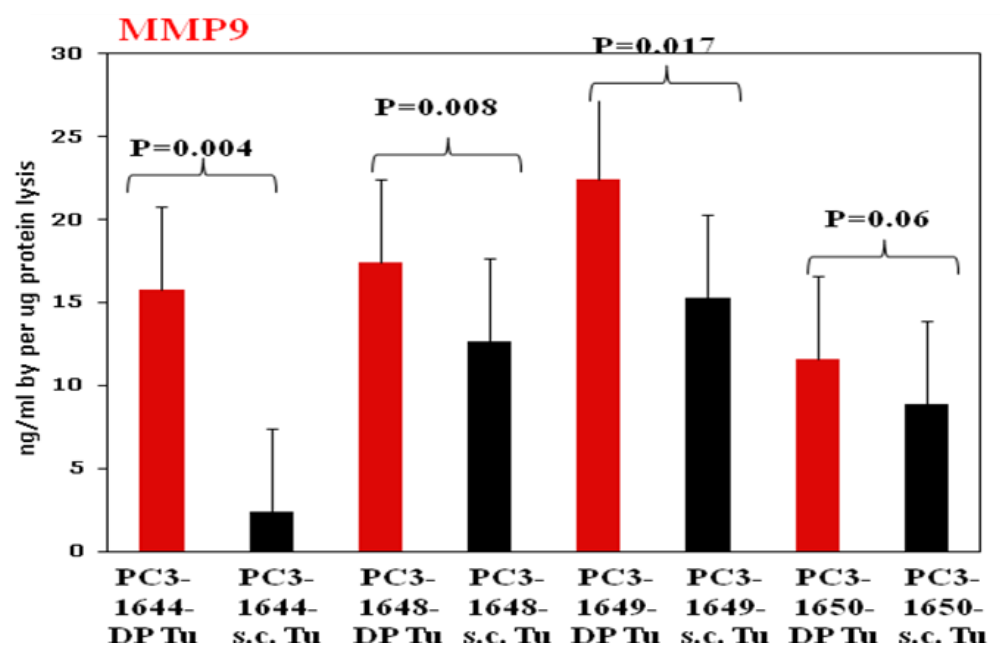

Figure 4.9. OPN WB in LAPC9 DP/sc tumor samples

(A) LAPC9 tumor WB for OPN expression in DP and s.c. pairs. (B) OPN WB of DU145 DP and s.c. tumor pairs. (C) OPN ELISA from PC3 DP/sc tumor samples (D) OPN ELISA from PC3 $\mathrm{DP} / \mathrm{sc}$ tumor bearing mouse plasma (E) MMP9 ELISA from PC3 DP/sc tumor samples. 
other hand, since the cells in either DP tumors or sc tumors are initially identical, the difference in CSC feature between them caused by different microenvironments suggests that CSCs may be a dynamic or transition population, not a fixed population. As long as they are in an appropriate niche, cancer cells may be modified to enter a status of CSC and to perhaps enrich metastatic potential.

We further validated the differentially-expressed genes by real-time RT/PCR, IHC and/or Western blotting (Figure4.8, 4.9). We identified eight up-regulated genes and two downregulated genes from the Human Microarray on which to perform qRT-PCR analysis. The results correlated exactly with the microarray data. DP/sc pairs from animals 714 and 717 showed a much higher up-regulation than that of animal 718 in our microarray, and we observed a similar high fold change in mice 714 and 717 compared with that of mouse 718 in our qRT-PCR (Figure 4.8 A). Moreover, we also investigated another gene, TGF- $\beta$ 1, that did not show up in our list of commonly upregulated genes. We only detected a slight change (1.3 to 1.9 in all three pairs) by qRT-PCR.

The perfect match between microarray and real time PCR confirmed the accuracy of our gene microarray. In addition, we verified OPN, IGFBP5, MM9, CXCR4, and TGF- $\beta$ - 2 by IHC analysis and the results revealed increased protein expression for all five molecules in the DPgrown PC3 tumors (Figure 4.8B). This gene signature is not evident in PC3 alone, as OPN was found to be overexpressed in DP tumors compared with sc tumors in LAPC9 and DU145 ( Figure 4.9 A). Additionally, because both OPN and MMP9 are secreted proteins, we carried out ELISA tests on either these tumor samples or tumor-bearing plasma. Both tumor samples and plasma showed the same trends which correlate with our microarray data (Figure 4.9). 


\section{The CSC Stem Cell Signature Was Validated in Other PCa Lines by Superarray}

Since our microarray mainly used PC3 as the experimental model, we wanted to verify our gene signature in other prostate cancer lines including LAPC9, DU145 and LNCaP, as well as two highly metastatic cell lines LM1 and BM1 that were derived from PC3 DP distant organ metastasis. We designed a stem cell gene signature-based super array to test if this stem cell signature is commonly overexpressed in other prostate cancer DP models, as well as those metastatic cells recovered from distant organ metastasis. These experiments are currently ongoing.

\section{Genes Up-regulated in the DP Tumors Are Functionally Involved in PCa Metastasis}

Since PCa cells grown in the mouse DP demonstrate a unique gene expression pattern which is highly related to metastasis, it suggests that these genes play critical roles in regulating metastasis. Therefore, we used shRNA-based knockdown assays to check the metastatic function of these genes. We chose OPN (one of the highest up-regulated genes in DP tumors), MMP9, ESM1 (another one of the highest up-regulated genes in DP tumors), TGF- $\beta$ 1, TGF- $\beta 2$, IL-1 $\beta$ , and CD44 as our targets. Since shRNA knockdown inhibition is dependent on shRNA targeting one or several sequence fragments of target genes, shRNA vectors usually have several isoforms to target different sequences of one single gene. Hence, we used a shRNA vector package including all multiple isoforms. After transfection, cells were cultured with puromycin to select transfected cells. These knockdown lines were close to $100 \%$ transfected according to the GFP 
positive cell percentage. The knockdown effect was substantiated by qRT-PCR(data not shown). A non-silencing vector (NS) was used as a negative control. In order to check metastasis, we injected 500,000 cells into the mouse DP for each line. After 40 days, primary tumors were harvested and metastases were checked in lung, pancreas, spleen, kidney, renal lymph node, liver and brain, again using lung metastasis as the overall indicator of tumor dissemination. By checking the tumor weight and metastases, we did not see a difference in tumor weight and tumor rate between the NS line and knockdown lines (Figure 4.10 A). However, lung metastases were significantly reduced in several lines including OPN2, TGF- $\beta$ 2, CD44-3, and MMP9 regardless of tumor size (Figure 4.10 B, 4.11 E, 4.12). In addition to these in vivo assays, we performed invasion assays by knockdown of MMP9 in a C-4-2 HPCa line model. Both invasion percentage and total invaded cells significantly decreased in knockdown lines (Figure $4.11 \mathrm{~A}-\mathrm{C}$ ). We further used the OPN-neutralizing antibody to perform the invasion assay. We found that the OPN antibody impaired the invasion ability of PC3 cells in vitro (Figure 4.12D). Overall, these results indicate that OPN, TGF- $\beta 2$, CD44, and MMP9 function as metastasis-regulating genes. It suggests that our microarray reveals a functional gene signature. More importantly, these genes' knockdown did not affect the primary tumor's growth ability. This may be reasonable since sc tumors have high tumor growth ability compared with DP tumor and such tumor initiating/tumor growth related may be irrelevant in this gene signature. The reason why tumor growth failed to make a difference might be due to the large number of cancer cells injected, 
A

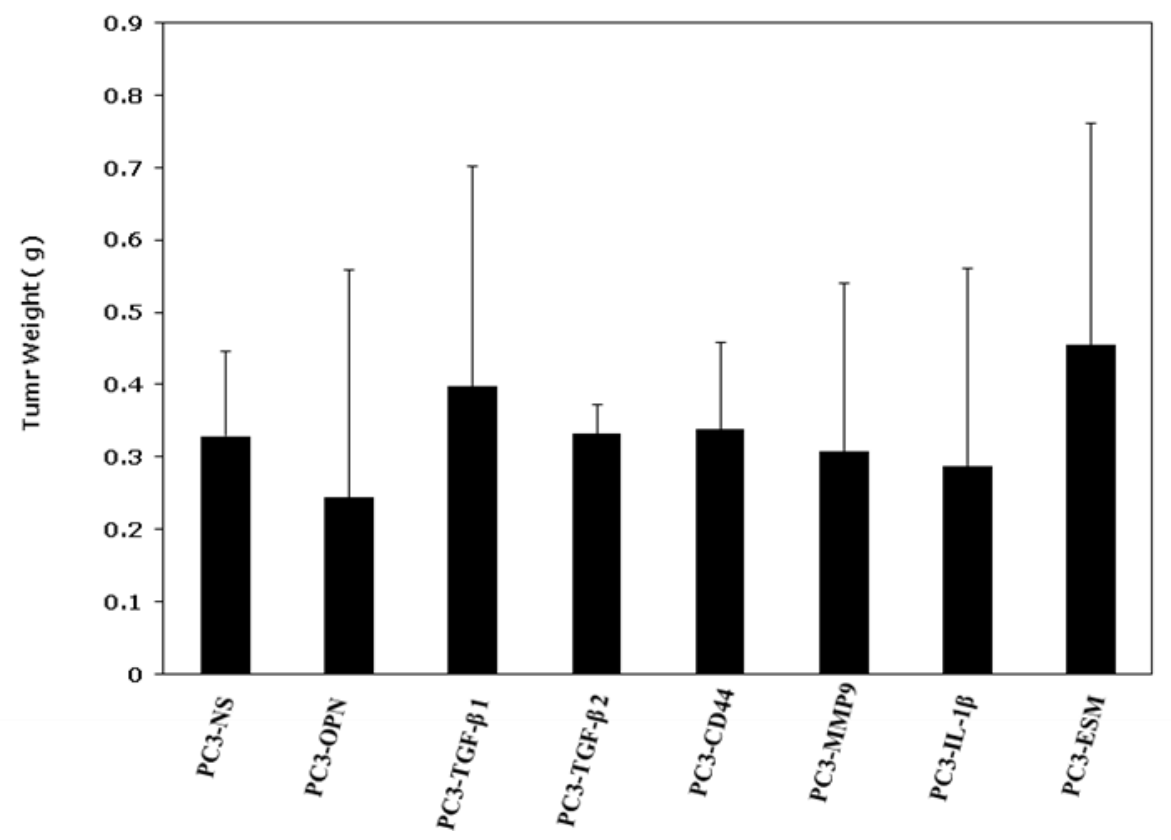

B

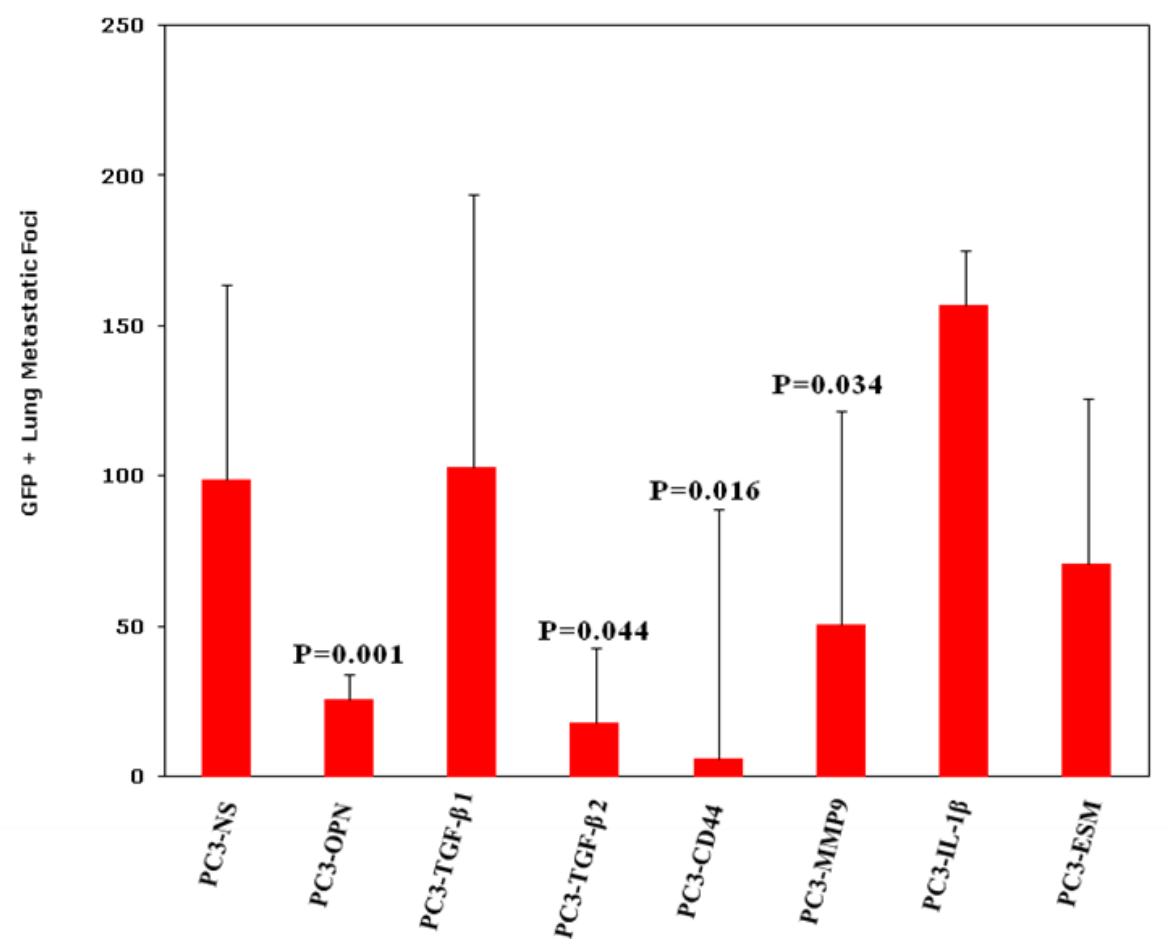

Figure 4.10 shRNA knockdown tumor experiments in PC3 model.

shRNA GIPZ vectors were from Open biosystem and injected into PC3 cells. For each target gene, a stable PC3-GIPZ cell lines were set up. (A) 500,000 cells of each PC3-GIPZ line were injected into mouse DP, after 40 days, tumors were harvested and measured the tumor rate and tumor weight. P value was calculated by compared with the PC3-non-slience control line injected tumors and listed on the column of each PC3-GIPZ lines. (B) Lung metastasis was checked as the representative of metastases. P vales were calculated by compared with NS control group. 


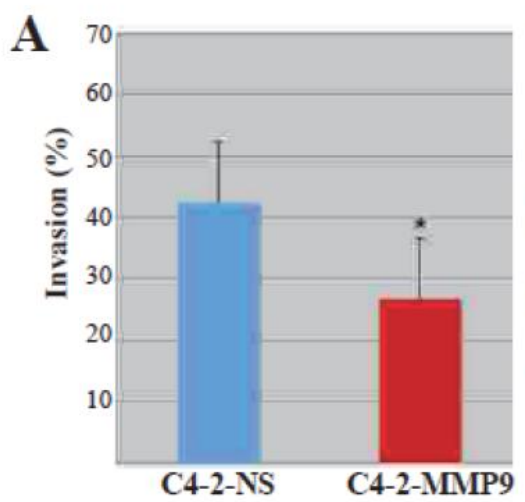

\section{B}
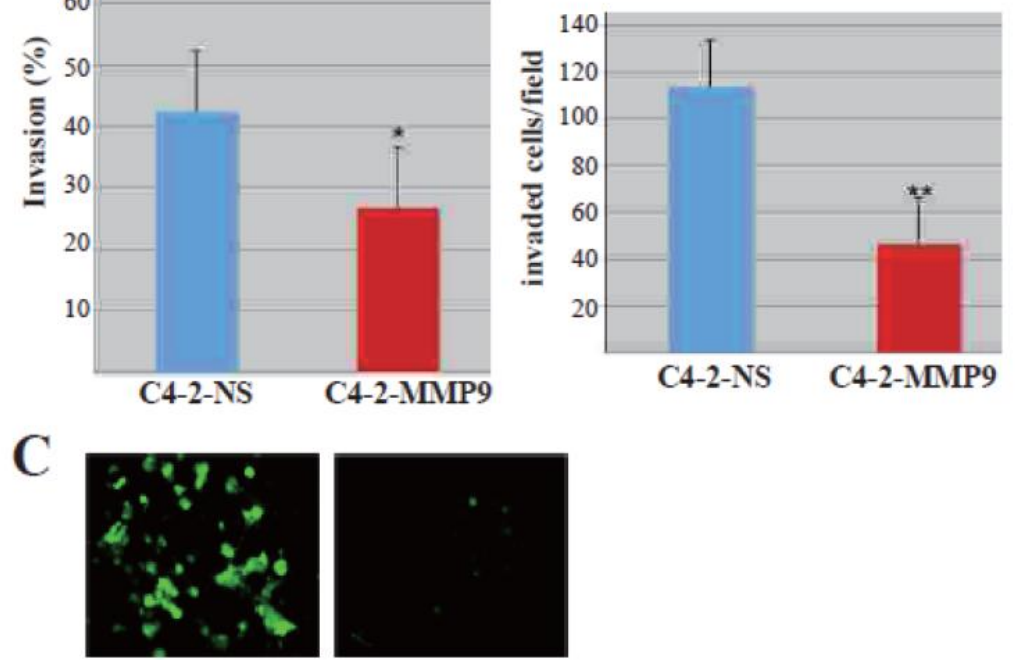

D
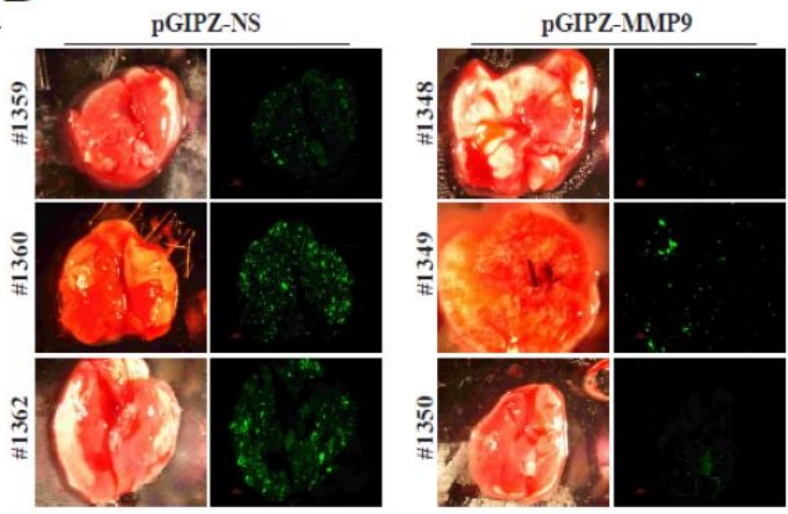

$\mathbf{E}$

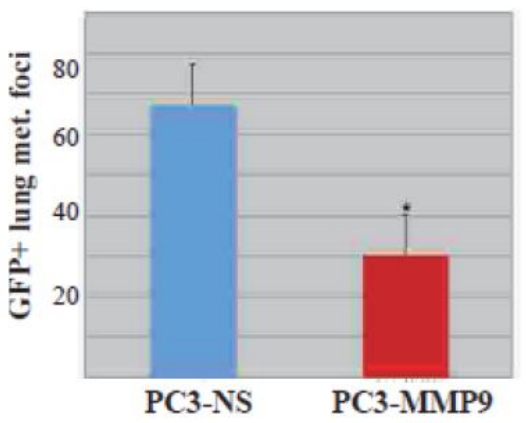

Figure 4.11 MMP9 is functionally involved in PCa cell invasion and metastasis

(A-C). MMP9 knockdown inhibits LNCaP C4-2 (C4-2) cell invasion. Log-phase C4-2 cells were infected with either the control pGIPZ lentiviral vector (which encodes an shRNA targeting a generic non-silencing [NS] sequence) or pGIPZ-MMP9-shRNA (MMP9)lentiviral vector. Both vectors were GFP-tagged and obtained from the Open Biosystems and used at an MOI (multiplicity of infection) of 25. Forty eight $h$ after infection, 10,000 cells were plated into one well of a Boyden chamber in which the $8 \mathrm{u}$ m porous membrane was pre-coated with Matrigel. Boyden chambers with non-coated 8-um membrane inset were used as control. Twenty four $\mathrm{h}$ after plating, the invaded cells were quantified (A-B) and imaged (C; left, pGIPZ-NS, right, pGIPZ-MMP9-shRNA). In A, the levels of invasion were plotted as $\%$ invasion relative to the control chamber inserts ( $* \mathrm{P}<0.05)$. In $\mathrm{B}$, invasion was presented as the numbers of invaded cells/20x field (**P $<0.01)$. A total of 10 random fields were counted for both conditions. (D). MMP9 knockdown inhibits PC3 cell metastasis. PC3 cells were infected with either pGIPZ-NS or pGIPZ-MMP9-shRNA (MMP9) lentiviral vectors (at MOI 25). 500,000 cells of each type were injected in 50\% Matrigel into the DP of NOD/SCID mice. Animals were terminated 40 days after implantation. Representative lung Images were shown in D ( 3 animals each) and GFP+ lung metastatic foci ( $>1 \mathrm{~mm} 3)$ were quantified $(\mathrm{E}, \mathrm{n}=5$ for each). $* \mathrm{P}<0.001$. 


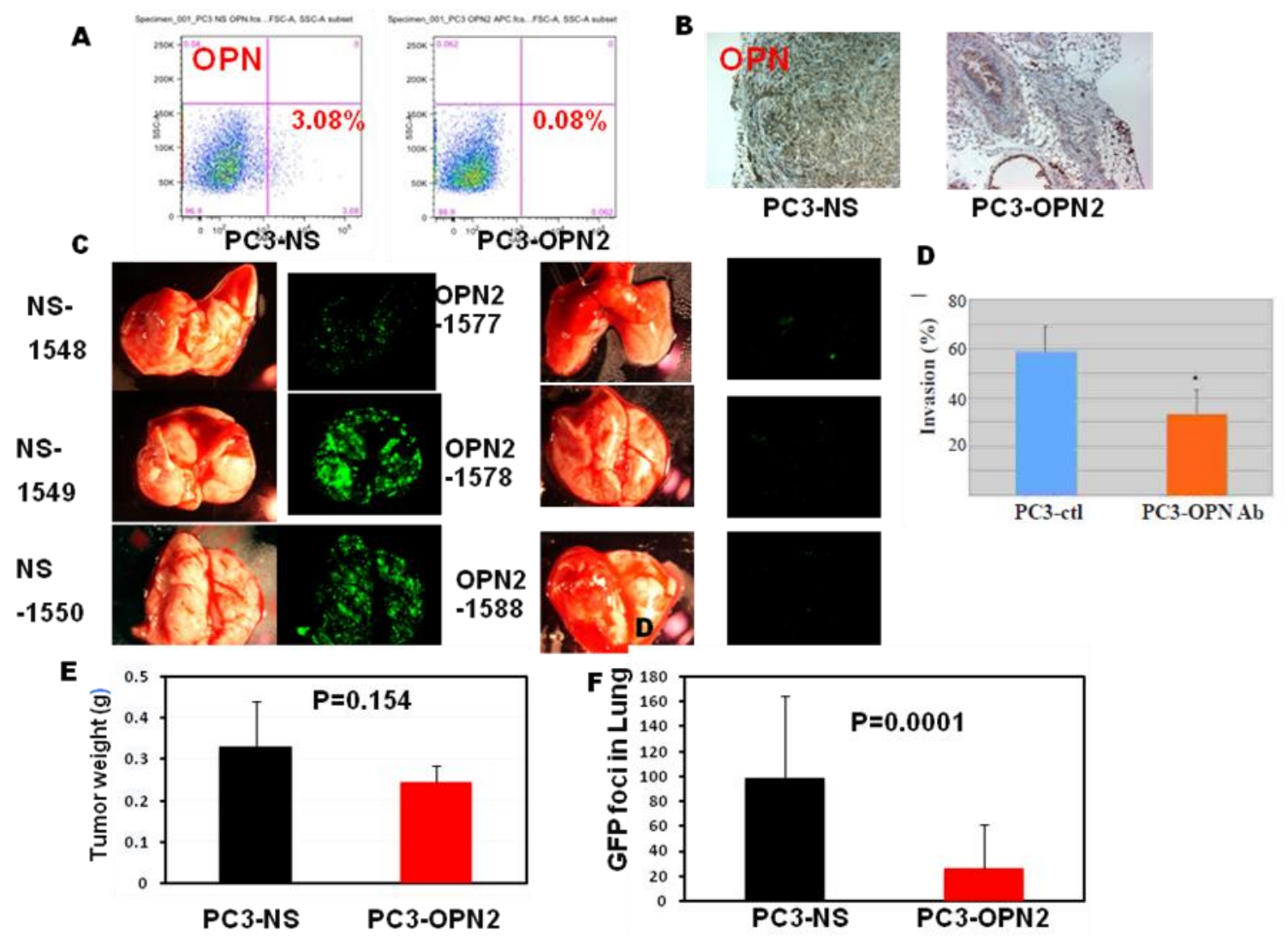

Figure 4.12 PC3-GIPZ-OPN2 Knockdown Xenograft model suppress metastasis

PC3 was infected with GIPZ-OPN vector. (A) The knockdown effect was checked by flow cytometry. PC3-GIPZ-OPN2 significantly reduced OPN expression compared with PC3-GIPZNS control. (B) IHC image showed the OPN knockdown tumor also decreased the expression of OPN. (C) Lung metastasis image of OPN knockdown effect. (D) Anti-OPN antibody blocks PC3 cell invasion. PC3 cells were preincubated with control $\mathrm{Rb}$ IgG or a $\mathrm{Rb} \mathrm{pAb}$ against OPN and then plated, in the presence of antibodies into the Boyden chambers. The results were plotted as $\%$ invasion as in $\mathrm{A}(* \mathrm{P}<0.01)$. (E) OPN knockdown did not suppress the tumor growth (measured by tumor weight) (F) OPN knockdown inhibited lung metastasis (average of group mice) of PC3 DP implantation. 
the metastatic behavior of these knockdown lines is significantly reduced regardless of difference in tumor size. It suggests that these metastatic genes are truly metastasis-specific and may play a critical role in regulating metastasis but not tumor growth. However, not all these genes from our gene signature can function as key regulators of metastasis. Some of them, like ESM1, though highly expressed in DP, are not able to suppress metastasis alone, and may only be a part of the metastasis machinery rather than significant modulators of metastasis.

\section{$\mathrm{OPN}^{+}$and $\mathrm{CD}^{+}{ }^{+}$prostate $\mathrm{CSC}$ function as key functional markers for regulating metastasis.}

Since the microarray data revealed a striking metastasis-related stem cell signature, the next question is "Do these cells function as CSCs, and furthermore, do these CSC signatures really modulate metastasis"? In order to answer these questions, stemness genes should pass the in vivo animal experiment to test metastatic ability. The most convincing way to do this is to isolate distinct CSC population by flow cytometry and inject these cells into the mouse DP. Among these widely reported CSC markers, we included six markers including CD133, ABCG2, CD44, OPN, CXCR4 and CD24 from our CSC gene signature. Preliminary data indicates that not all of these stem cell markers work well in regulating tumor growth and metastasis. Neither CD133, $\mathrm{ABCG}^{+}$, nor $\mathrm{CXCR} 4^{+}$exhibited a stronger metastatic phenotype and tumor growth ability than their negative counterparts (data not shown). It suggests that CSC regulation of metastasis may be complicated, or that these CSCs markers may just be one aspect of the metastatic regulation machinery. However, OPN, CD24, and CD44 stand out in this screen. Osteopontin is a secreted glycophosphoprotein and abundantly expressed in tissues during inflammation and repair 
(Brown et al., 1994). In tumor biology, OPN is widely linked to EMT, metastasis, inflammation and instigation (McAllister et al., 2008; Tuck et al., 2007). More interestingly, OPN is also involved in regulating CSCs: It is a negative regulator of HSC proliferation (Nilsson et al., 2005), the ligand of CSC marker CD44 (Weber et al., 1996), and a molecule which helps to build the pre-metastatic niche in distant organs (Li et al., 2007b). In our current study, OPN is one of the top upregulated genes in DP tumors. However, OPN as a surface protein was largely ignored for a long time. In fact, Verhulst et al successfully localized OPN at the apical surface in renal cells by flow cytometry recently (Verhulst et al., 2002), suggesting that stressed renal cells attract OPN and OPN enters the cell through endocytosis. During this process, OPN anchors itself on the cell surface. Based on this, OPN can be detected by flow cytometry in living cells in the same manner as a surface protein. A surface protein usually functions as a receptor to bind to the ligand and thus can be used as marker to purify distinct cell population. Though it is still unclear what is the mechanism of OPN localize in membrane, the idea to use OPN as a cell surface marker to isolate $\mathrm{OPN}^{+/-}$population in vivo is one we employed. Because OPN is the one of most up-regulated genes in DP tumors, it is significant to test the function of the $\mathrm{OPN}^{+}$ population in regulating metastasis and tumor development. There is still no single report to test it on cancer cell. Hence, in our current study, we first show that there are $4.93 \% \mathrm{OPN}^{+}$cells in PC3 culture by flow cytometry (Figure 4.13). Importantly, the expression of OPN is correlated to our microarray data in that OPN is two-fold higher in DP tumors than that in sc tumors in the LAPC9 model (12.6\% vs 6.22\%) (Figure 4.13). Shown in figure 4.13, we further purified OPN ${ }^{+}$ and $\mathrm{OPN}^{-}$cells from our PC3 culture and performed DP injections with three cell numbers: $1,000,10,000$, and 100,000. We harvested tumors after 8 weeks, and to our surprise, OPN ${ }^{+}$cells 
A

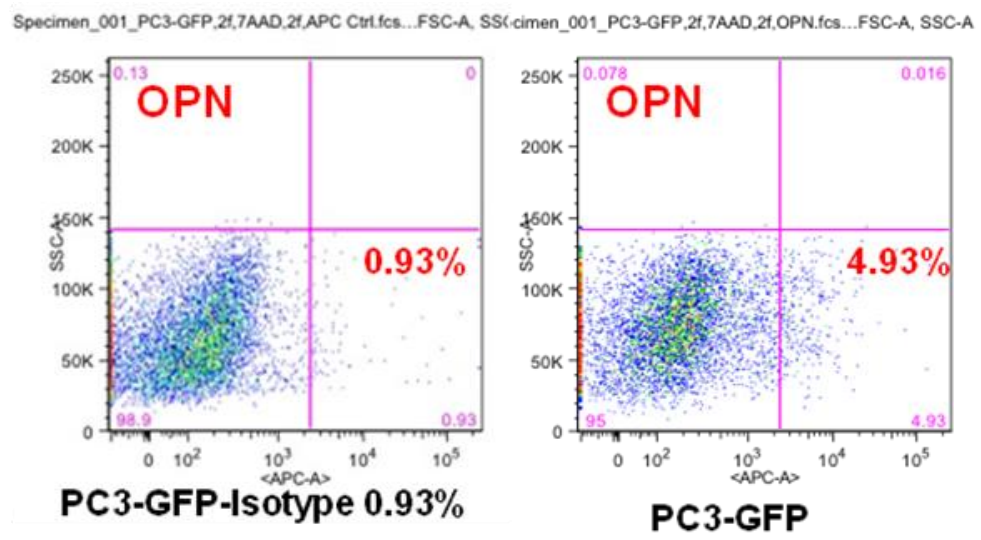

B
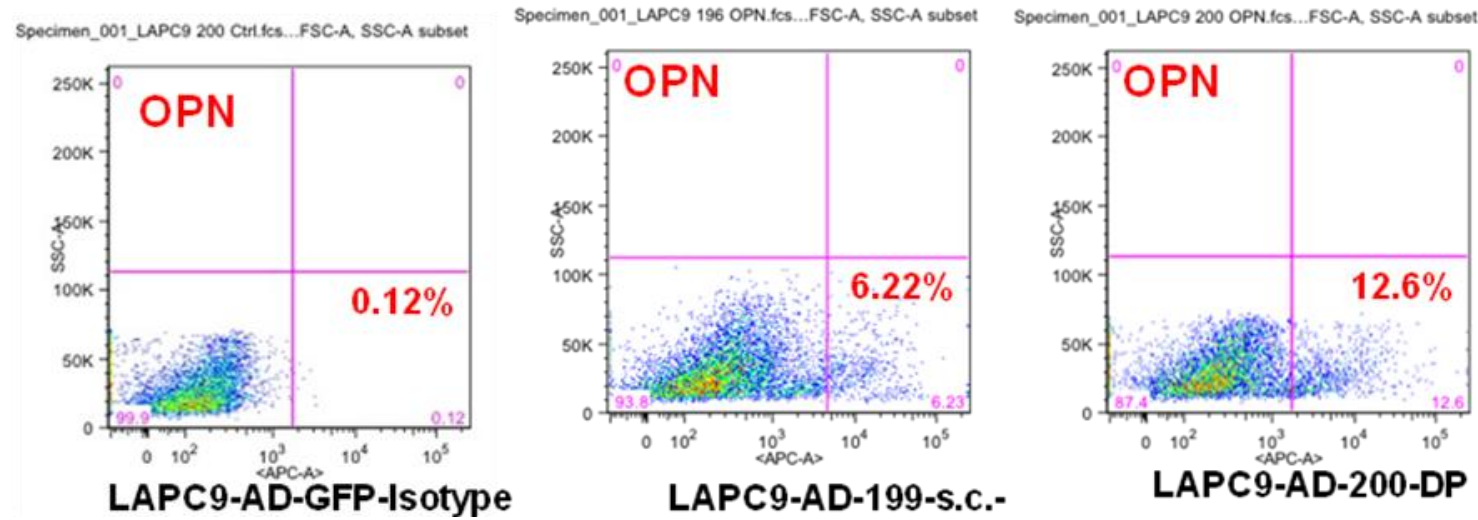

Figure 4.13 OPN expression in the cell surface of PCa.

(A)OPN is detected in live PC3 cells with a surface antibody by using flow cytometry. (B): LAPC9-AD tumors from DP and s.c. implant sites were harvested and single cell suspension were made according to the protocol described in methods and material. OPN is highly expressed in DP tumor ( $12.6 \%$ ) compared with s.c. tumor ( $6.22 \%)$. 
were dramatically more tumorigenic and metastatic than the $\mathrm{OPN}^{-}$population. $\mathrm{OPN}^{+}$tumors were evident even with as few as 1,000 cells injected, while $\mathrm{OPN}^{-}$cells only could initiate tumors in 1 out of 3 mice with 10,000 cells injected, yielding a tumor-initiating frequency (TIF) enrichment of almost 100 fold (1/910 in $\mathrm{OPN}^{+}$vs $1 / 83580$ in $\mathrm{OPN}^{-}$tumors). More importantly, $\mathrm{OPN}^{+}$cells are more metastatic, just as we expected. $\mathrm{OPN}^{+}$tumors with 10,000 cells injected show much strengthened lung metastasis compared to $\mathrm{OPN}^{-}$tumors with 10,000 cells injected. Although we did not expect OPN to influence tumor growth since our gene signature may only concern metastasis-related genes, we found that $\mathrm{OPN}^{+}$cells had a significant tumor growth advantage over $\mathrm{OPN}^{-}$cells. Not surprisingly, there were significantly more lung metastases in $\mathrm{OPN}^{+}$injected mice. However, knockdown of OPN by short hairpin RNA (shRNA) had no effect on tumor growth rate, but did significantly reduce lung metastases (Figure 4.14). This result indicates that OPN may not cause primary tumor growth advantage, but play a more critical role in regulating metastasis. Subsequent immunohistochemistry not only confirmed the knockdown effect by showing a decrease in OPN, but also a reduced expression of CD44 and the macrophage marker S100A9( data not shown). It suggests that OPN may be involved in regulating CD44 and immune cells. In addition, the in vitro invasion assay demonstrated that invasion ability was significantly decreased by application of neutralizing OPN antibody (Figure 4.12 D). These results imply that metastatic cancer stem cells might exist, and these metastatic cancer stem cells are not only specifically controlling metastasis, but are also involved in primary tumor growth. Additionally, it suggests that OPN has a greater influence in regulating metastasis than tumor growth. Our studies highlight OPN as a very promising marker for future CSC studies. 
A

\begin{tabular}{cccc}
\hline Injection cell \# & OPN $^{+}$ & OPN $^{-}$ & Pvalue \\
\hline 1000 & $1 / 3$ & $0 / 2$ & \\
10,000 & $3 / 3$ & $1 / 3$ & \\
100,000 & $1 / 1$ & $1 / 2$ & \\
TIF & $1 / 910$ & $1 / 83580$ & $\mathrm{P}=0.0338$ \\
\hline
\end{tabular}
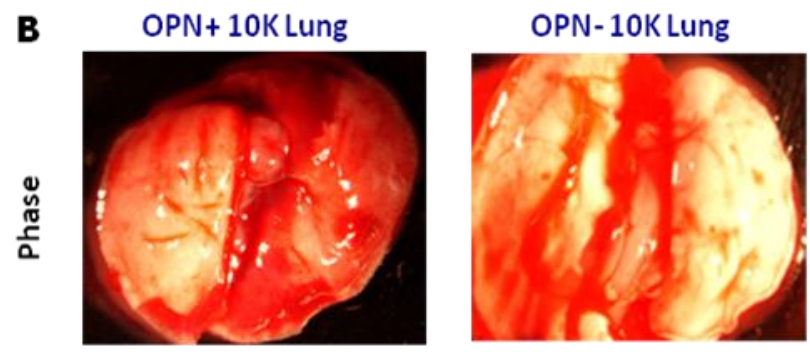

C
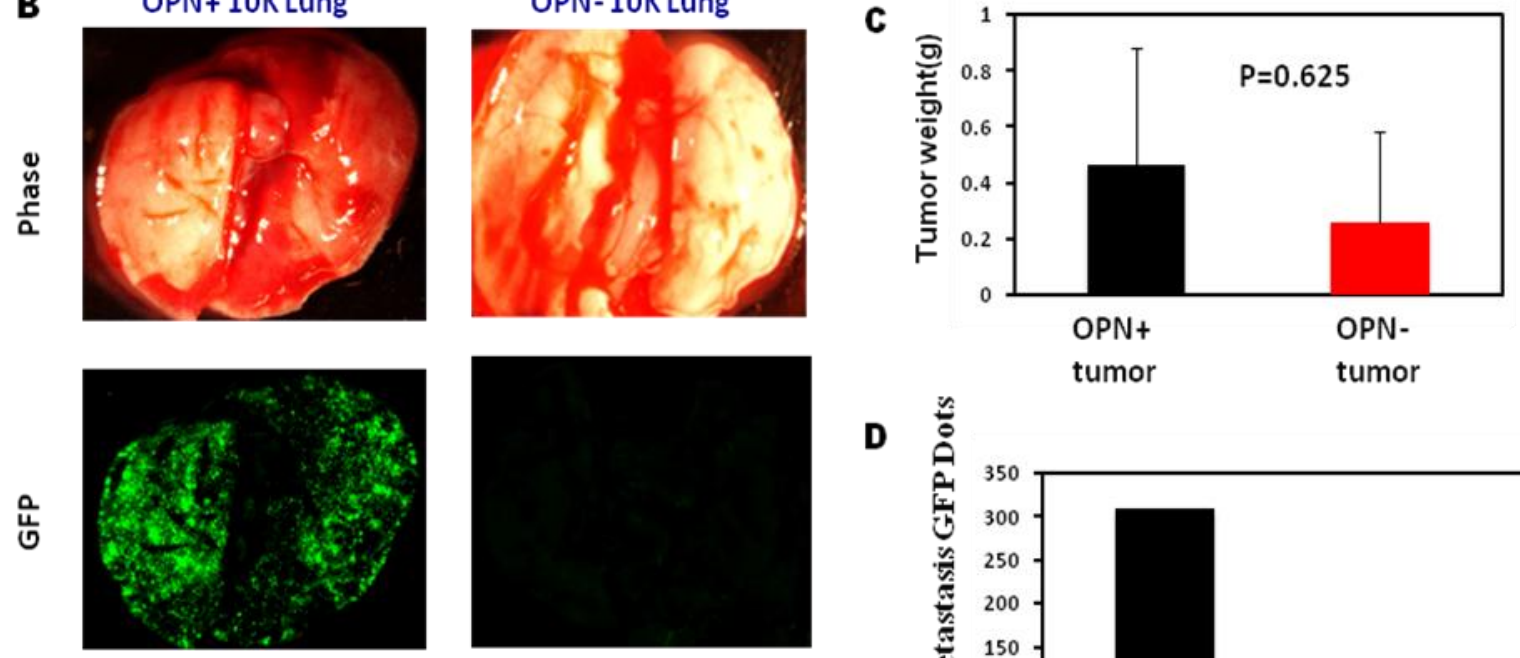

D

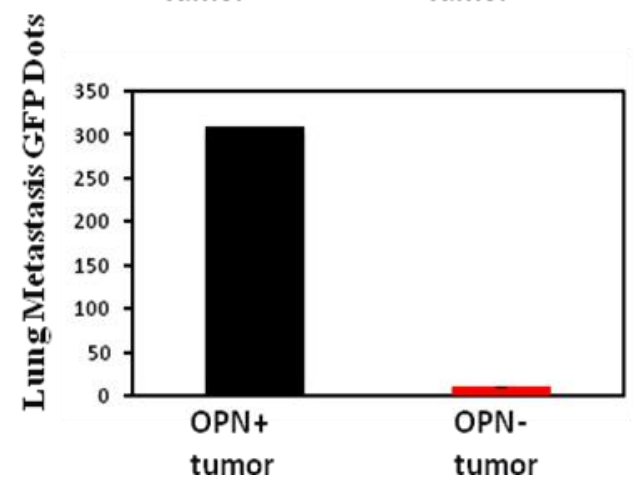

Figure 4.14: $\mathrm{OPN}^{+}$cells exhibit strong tumor growth and metastasis potential compared with $\mathrm{OPN}^{-}$counterpart

We sorted the $\mathrm{OPN}^{+}$and $\mathrm{OPN}^{-}$subpopulations from PC3-GFP cells by using flow cytometry. Then we injected these two populations into mouse DP respectively by limiting dilution. (A) Summary table for number of DP injection, tumor rate and TIF for $\mathrm{OPN}^{+}$and $\mathrm{OPN}^{-}$ subpopulations. (B) Representative lung metastasis image from $\mathrm{OPN}^{+}$and $\mathrm{OPN}^{-} 10,000$ cells injected mice. (C) Tumor weight between two groups. (D) Lung metastasis dots bar graph from $\mathrm{OPN}^{+}$and $\mathrm{OPN}^{-}$groups. 
The use of CD24 as a CSC marker is controversial and is dependent on tumor type: In breast cancer, including the first report of CSCs in solid cancer, Michael Clarke's group showed that CD24 actually is low or negative in CSC populations, instead using $\mathrm{CD} 44^{+} / \mathrm{CD} 24^{-}$as $\mathrm{CSC}$ markers (Al-Hajj et al., 2003). However, in pancreatic cancer and more recently in colon cancer, $\mathrm{CD}_{4} 4^{+} / \mathrm{CD} 24^{+}$were used as CSC markers (Li et al., 2007a). CD24 is correlated with tumor progression in these tumors (Yeung et al.). More interestingly, in human prostate cancer, CD24 over-expression was found to be linearly related to prostate cancer development and progression (Kristiansen et al., 2004). Therefore, we analyzed CD24 expression in PC3 cells (21.05\%), while PC 3-717 DP( a DP tumor-derived cell line) has $23.04 \%$ compared with $11.53 \%$ of PC3-717-sc ( a sc tumor derived cell line) ( Figure 4.15). We isolated CD24 positive and negative cell populations from PC3-GFP cells respectively by using flow cytometry. These cells were then implanted into mouse DP in a series of ten, 100,1000, 10,000 and 50,000 by limiting dilution ( see Figure 4.16). After 8 weeks, we harvested the tumor, and tumor rate, tumor weight and metastasis were checked. It is very interesting that $\mathrm{CD} 24{ }^{+}$failed to exhibit a tumor growth advantage compared with its negative counterpart in DP injection. The TIF in CD24- tumors is four-fold that of $\mathrm{CD} 24^{+}$tumors ( $1 / 339$ vs $\left.1 / 1617\right)$. The tumor weight of $\mathrm{CD} 24^{-}$tumors is correspondingly more than that of positive tumor though the statistical significance is not obvious. It has been suggested that CD24 alone may not be a " good" CSC marker but may need to be combined with other stem cell markers in tumor development. Strikingly, however, CD24 exhibits a strong ability to regulate metastasis. Lung metastasis is dramatically reduced in the $\mathrm{CD} 24^{-}$group compared with the $\mathrm{CD} 24^{+}$group though the weight of $\mathrm{CD} 24^{-}$tumors is much 
A

Spocimen_001_PC3 GFP APC.fCs...FSC.A SSC.A subeot

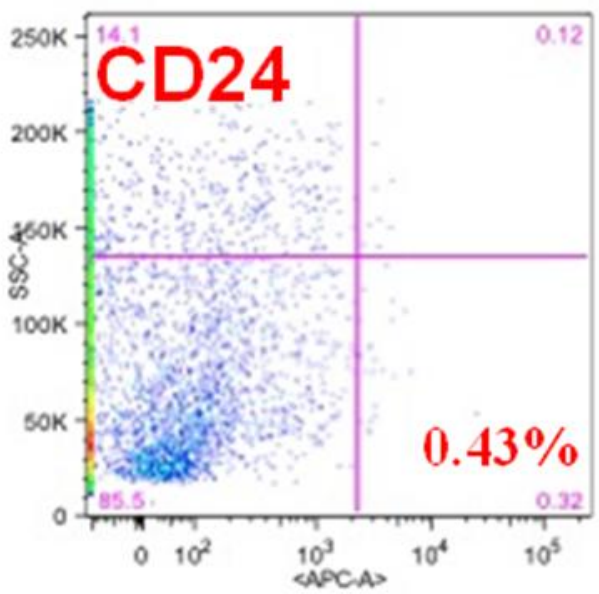

C

PC3-GFP-Isotope

Specimen_001_717 DP CD24.fos..FSC.A. SSC.A ubset

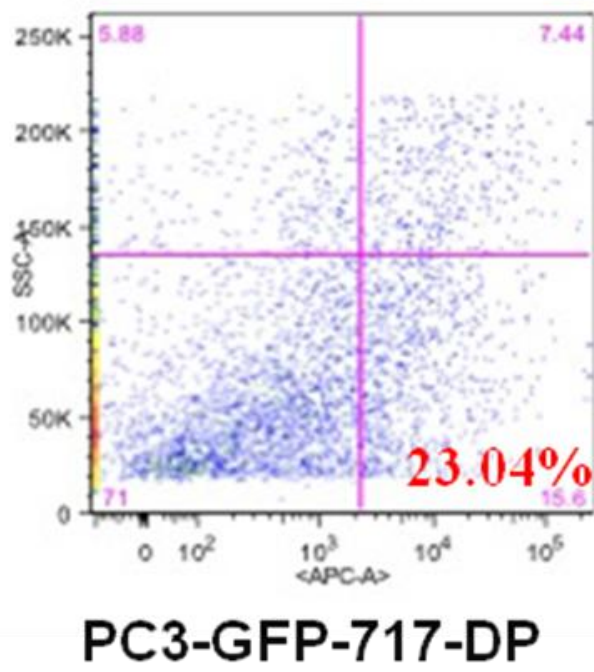

B

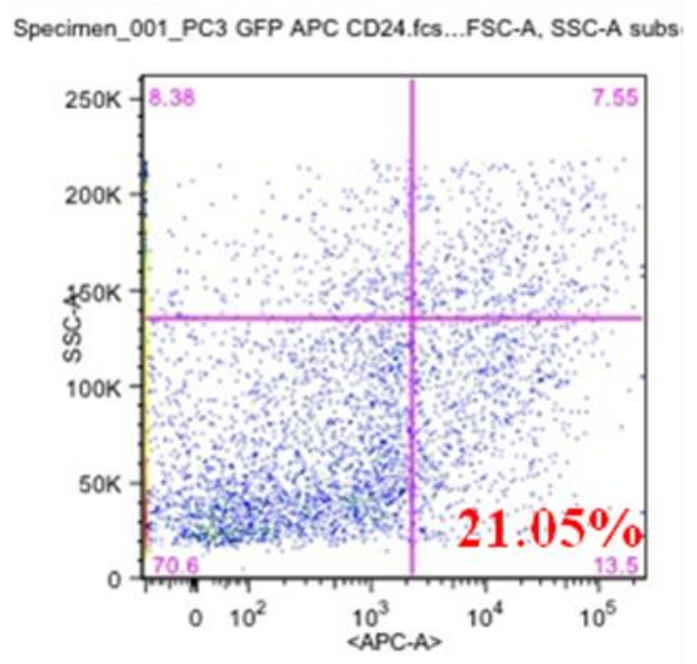

D

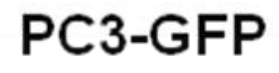

Specimen_001_717 SQ CD24.fcs...FSC-A, SSC-A subset

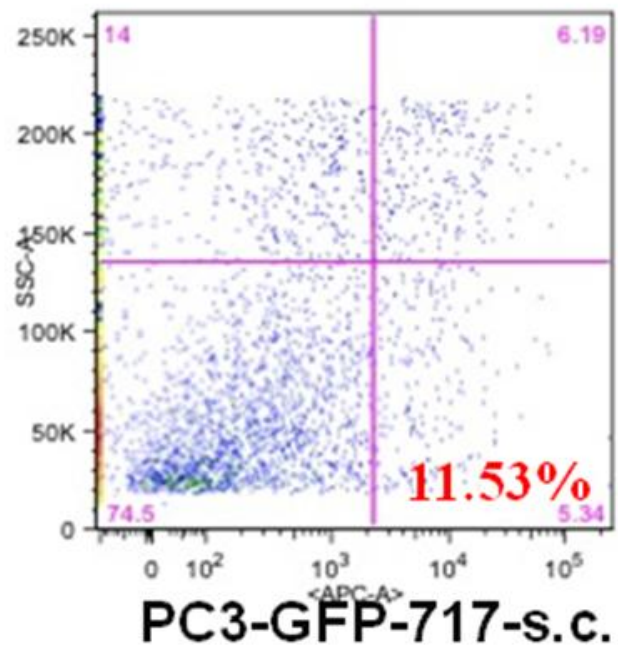

Figure 4.15 CD24 expressions in the cell surface of PCa cells by detection of flow cytometry.

CD24 expression was up-regulated in a PC3-DP derived line $(C)$ compared with a s.c. derived lines (D). (A) is the isotope control (B) CD24 expression in PC3 cells. 
$\mathbf{A}$

\begin{tabular}{cccc}
\hline Injection cell \# & CD24 $^{+}$ & CD24 & Pvalue \\
\hline 100 & $0 / 2$ & $0 / 1$ & \\
1000 & $1 / 2$ & $2 / 2$ & \\
10,000 & $2 / 2$ & $2 / 2$ & \\
50,000 & $1 / 1$ & $1 / 1$ & \\
TIF & $1 / 1617$ & $1 / 339$ & $\mathrm{P}=0.554$ \\
\hline
\end{tabular}

B CD24+1000 Lung CD24-50000 Lung
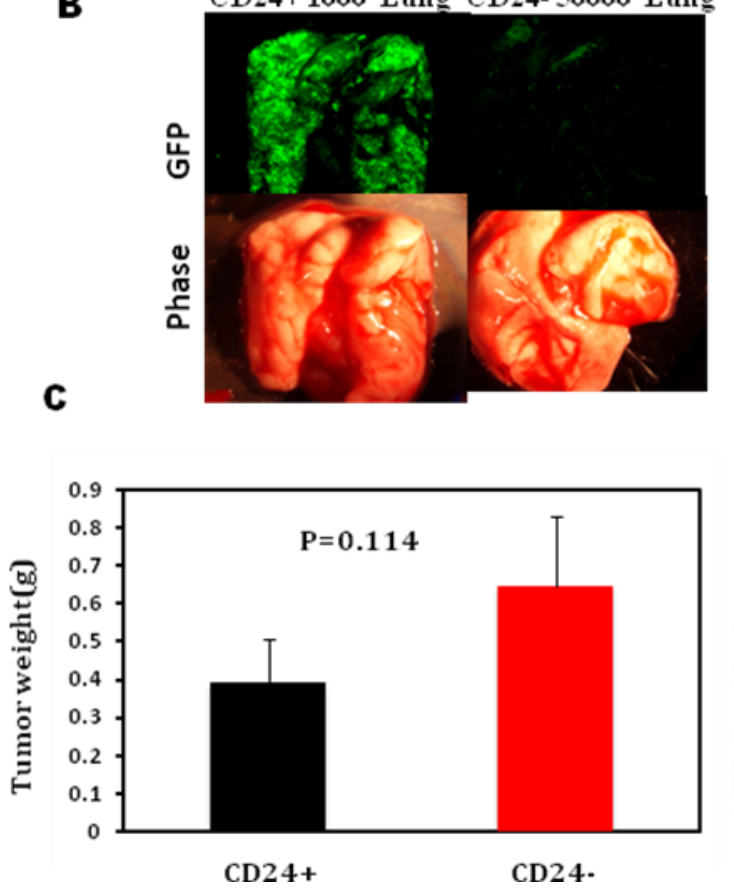

D

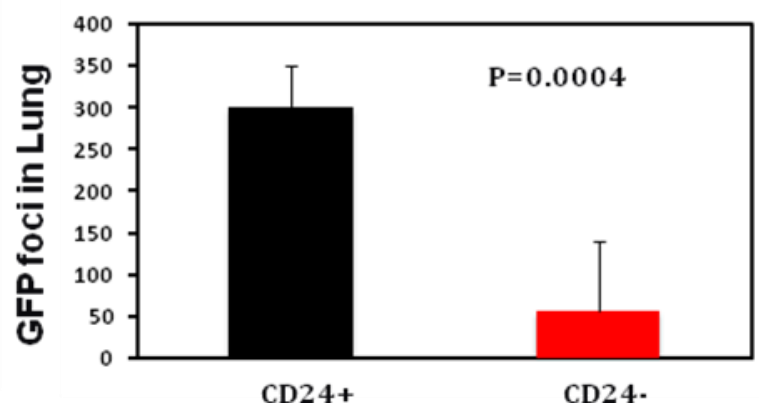

Figure 4.16 PC3-GFP CD24 ${ }^{+}$population enriched metastasis potential

(A) Purified PC3-GFP CD24 $4^{+}$and CD24 populations were injected into the mouse DP according to limited dilution. Tumor rate and TIF were summarized in this table. It suggests that CD24population may hold stronger tumorigenesis ability than $\mathrm{CD} 24^{+}$population. (B) $\mathrm{CD} 24^{+}$ population enhances metastasis. The lung metastasis in 1,000 cells injected animal is much more than 50,000 cells injected animal in CD24 population. (C) Tumor weight is no difference between these two groups. (D) Lung metastasis (average of group mice) was significantly reduced in CD24- group. 
A

\begin{tabular}{cccc}
\hline Injection cell \# & CD24 $^{+}$ & CD24 & Pvalue \\
\hline 100 & $2 / 2$ & $0 / 1$ & \\
1000 & $3 / 3$ & $2 / 3$ & \\
10,000 & $3 / 3$ & $2 / 3$ & \\
TIF & $100 \%$ & $1 / 4688$ & $\mathrm{P}=0.554$
\end{tabular}
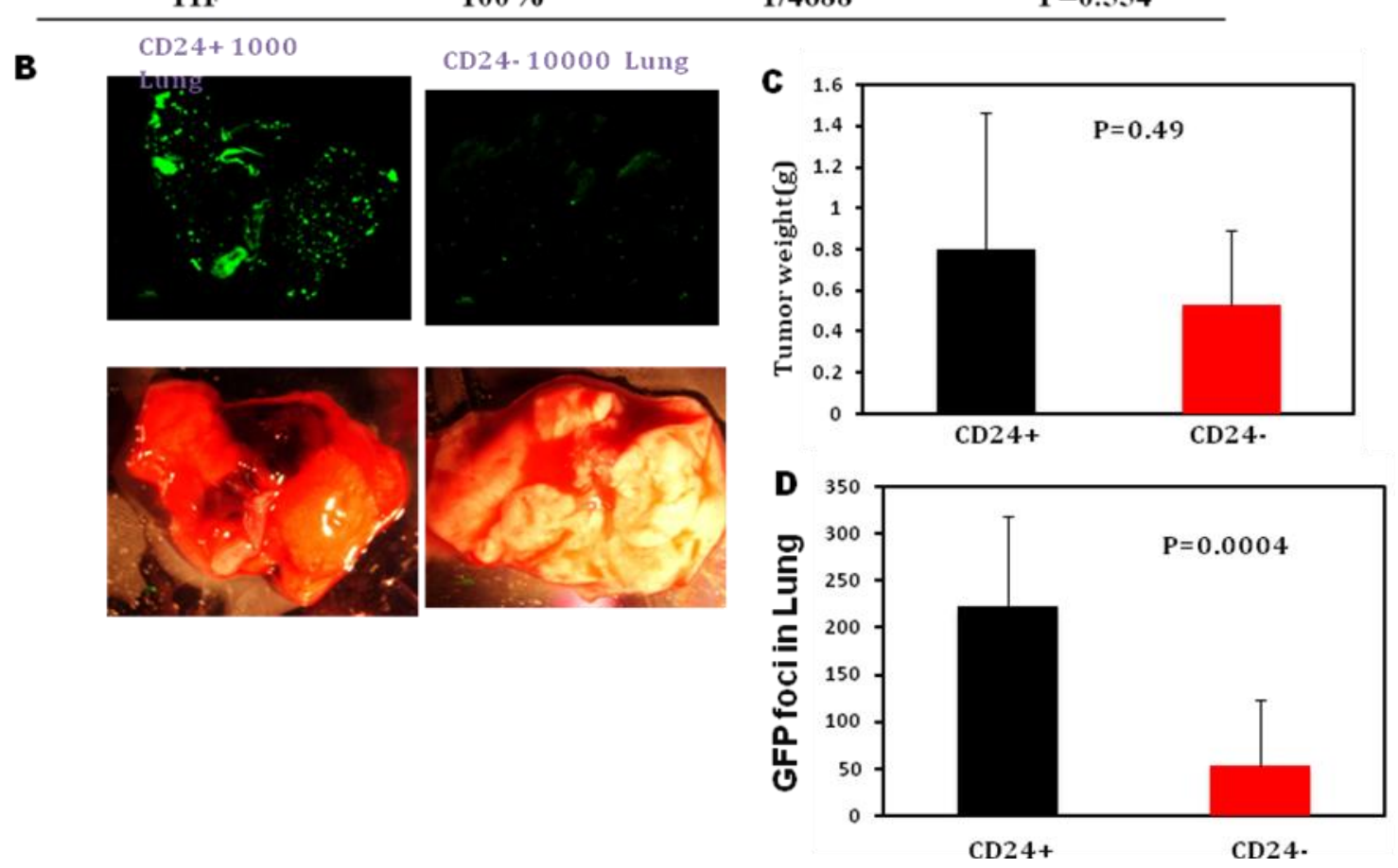

Figure 4.17 LAPC9-AI-GFP-DP CD24 +/- tumor and metastasis

(A)Purified LAPC9-AI-GFP CD24 ${ }^{+}$and CD24 populations were injected into the mouse DP according to limited dilution. Tumor rate and TIF were summarized in this table. (B) CD24 ${ }^{+}$ population enhances metastasis. The lung metastasis in 1,000 cells injected animal is much more than 10,000 cells injected animal in CD24- population. (C) Tumor weight is no difference between these two groups. (D) Lung metastasis (average of group mice) were significantly reduced in CD24- group. 
greater. We verified previously that there are more CD24 $4^{+}$cells in DP tumor than in sc tumors (Figure 4.15). Hence, we used LAPC9, an AR-positive prostate cancer line, derived from a patient bone metastasis and maintained in vivo, to test the tumorigenic and metastatic ability of the CD24-selected subpopulation (Figure 4.17). Surprisingly, the CD24 ${ }^{+}$subpopulation in LAPC9 did show a tumor growth advantage over the CD24- subpopulation both in TIF (100\% in $\mathrm{CD}_{2} 4^{+}$vs $1 / 4688$ in $\mathrm{CD} 24^{-}$) and tumor weight. However, the metastatic profile in LAPC9 is still highly consistent with the situation in PC3 tumors in that CD24 $4^{+}$tumors develop much more metastasis than $\mathrm{CD} 24^{-}$tumors. Even the injection of $1,000 \mathrm{CD} 24^{+}$cells results in significantly more metastasis than the injection of $10,000 \mathrm{CD} 24^{-}$tumors. These results imply that CD24 is a potential metastatic regulator regardless of tumor type. CD24 might function as marker of either a metastatic CSC or progenitor cells in some specific tumor types. The ability of CD24 to regulate tumor growth may be tumor type and cell type-dependent.

\section{Distant organ metastatic cells are enriched in CSC properties.}

Metastasis was found to be highly enriched by a multiple round injection model in breast cancer(Minn et al., 2005). Though previous studies reported that distant metastasis samples were enriched in CSC markers, whether these recovered metastatic cells from distant metastatic organs are enriched in CSC properties is still unknown (Balic et al., 2006). Since we have already provided evidence in the previous section to show that CSCs may be linked to metastasis in primary tumors, it might be very interesting to address the question of whether CSC properties could be enriched in these metastasis enriched cancer cells. In order to answer 
this question, we created a metastasis-enriched model by using the DP model (see Figure 4.18). First we injected bulk cultured PC3-GFP into mouse DP; after 8 weeks, we harvested the end organs, made single-cell suspensions, and cultured the disaggregated cells in PCa cell culture medium. Two weeks later, since most of the host mouse cells (either epithelial cells or support cells) died, we could observe only $\mathrm{GFP}^{+}$PCa cell colonies (Figures 4.19). These data also confirm that these metastatic cells are live cells in the distant organs which have the potential to form a local proliferative growth. Using similar strategies, we also observed that the GFPlabeled PC3 (Figure 4.19) or Du145 (not shown) cells recovered from multiple end organs including lung, tibia and femur supported robust clonal outgrowth in 1-2 weeks. After we successfully recovered these metastatic cells from distant organs, we tested whether or not these metastatic cells are also enriched in CSC properties. We re-injected lung metastasis cells (LM1) and Bone Marrow metastasis cells (BM1) into mouse DP and observed tumor growth and metastasis (Figure 4.20). Very surprisingly, these first-generation recovered metastatic cells show a great tumor growth and metastatic advantage compared to their parental cells. These cells could initiate tumor development and metastasis with as few as 100 cells (Figure 4.20 A). The TIF in both LM1 and BM1 is almost $100 \%$, which is much higher than that of their parental TIF, either with DP or sc injection. Moreover, these tumors also show a strong metastatic profile. This enrichment is so strong that the enrichment was achieved in just two generations. We believe these cells may be CSCs or metastatic CSCs due to their enriched tumor growth and metastasis advantage. Additionally, this enriched stem-like cell phenomenon was also observed in DU145 cells (data not show). 


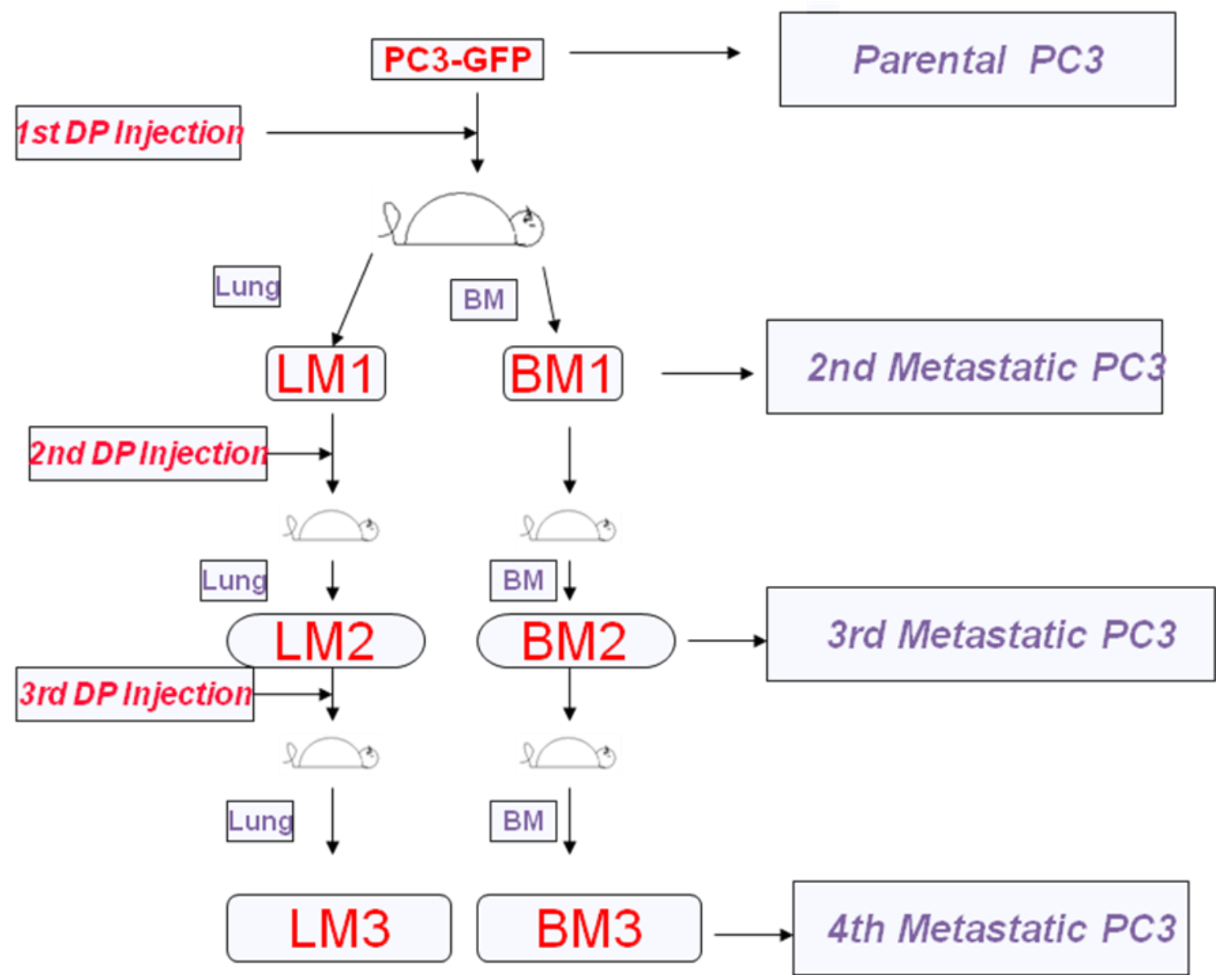

\section{Figure 4.18 Flow chart for serial enrichment PC3 Model}

PC3-GFP metastasis enrichment model: 2 million of PC3-GFP were injected into mouse DP, after 8 weeks, lung were harvested and digested into single cells suspension. Then culture in dish for 2-3 days, wash the dish and continue to culture these tissues with cancer cells favored medium. After 1-2 weeks, only human epithelial cancer cells were left and extend in the culture in most of cases. Purified these cells by flow cytometry and these cells are called LM1. Reinject these LM1 into mouse DP and form the second round enrichment. Since we get highly metastatic LM1 cells, we stop here and did not do the third round injection. BM1 were harvested by the same way but using the bone fusion to get the BM cells. 

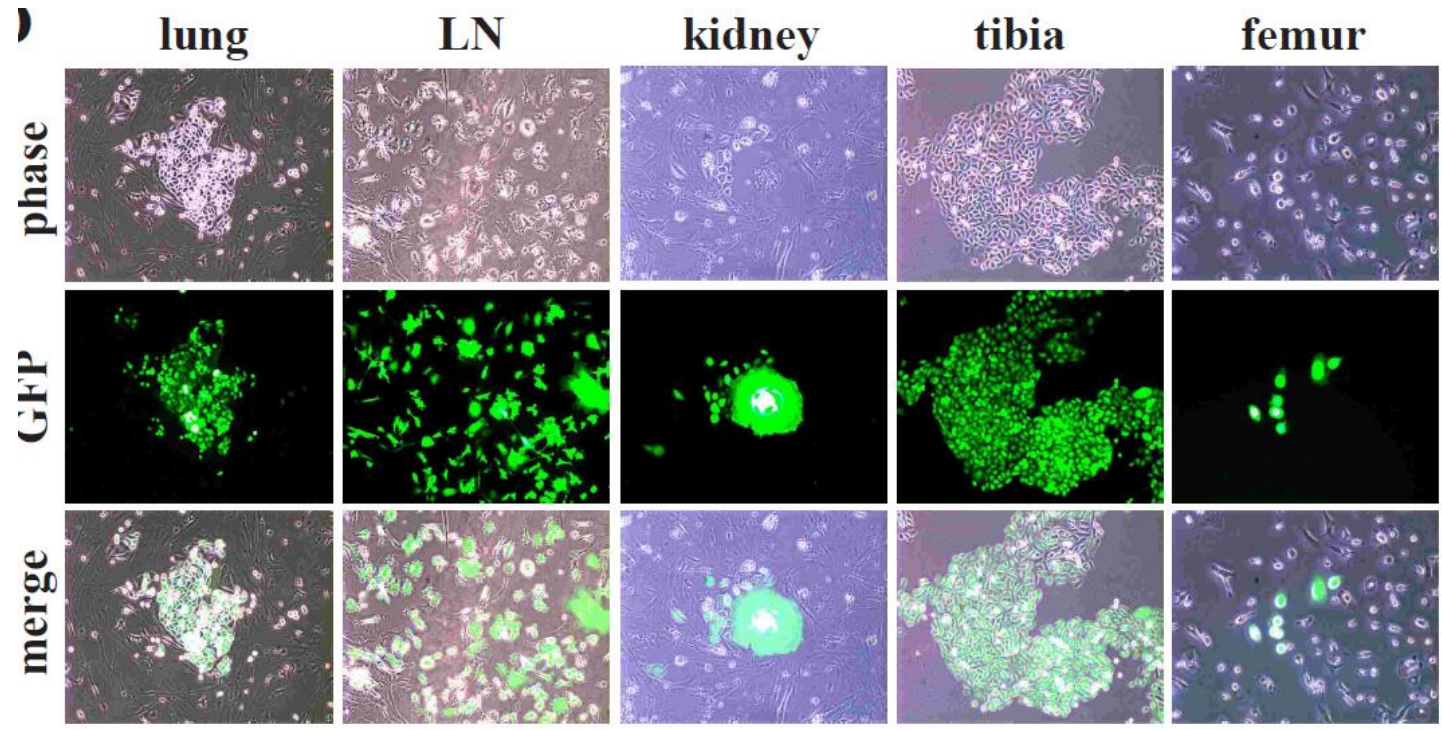

Figure 4.19 DP-implanted PC3-GFP cells at high cell numbers (i.e., 2,000,000 cells) metastasize to multiple organs

Shown are representative images of PC3-GFP cells cultured ( 2 weeks after plating) from various end organs. The GFP- cells on the background are the host cells. Original magnifications, $\mathrm{x} 200$ 
Second-round Metastatic PC3-GFP Enriched stem cells trait

A

\begin{tabular}{lclll}
\hline Cell Number & \multicolumn{2}{c}{ Tumor Rates } & & \\
& PC3 - s.c & PC3-DP & LM1 & BM1 \\
\hline 100 & $1 / 8(33)$ & -- & $3 / 3(46)$ & $2 / 2(51)$ \\
1,000 & $5 / 8(33)$ & $0 / 3$ & -- & - \\
10,000 & $8 / 8(18)$ & $2 / 4(107)$ & $3 / 3(41)$ & $1 / 2(52)$ \\
100,000 & $7 / 8(18)$ & $3 / 3(66)$ & $3 / 3(32)$ & $3 / 3(46)$
\end{tabular}

B
\begin{tabular}{|lcccc|}
\hline Cell Types & Lung & BM & LN & Multiple organs \\
\hline LMI-100 & ++ & - & +++ & $\pm \sim+++$ \\
LMI-10,000 & +++ & \pm & +++ & $\pm \sim++$ \\
LMI-100,000 & +++ & $\pm \sim+$ & +++ & $+\sim+++$ \\
BM1-100 & ++ & - & ++ & -+++ \\
BM1-10,000 & ++ & ND & ++ & $-\sim+$ \\
BM1-100,000 & ++ & $\pm \sim+$ & ++ & -+++ \\
& & & & \\
PC3 DP-1x 10 & +++ & -+ & $+\sim+++$ & -+++ \\
\hline
\end{tabular}

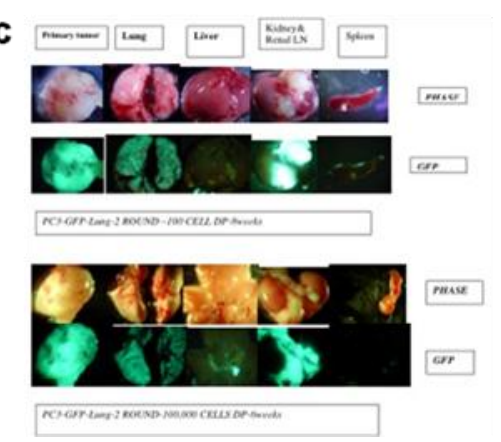

Figure 4.20 Second round Metastatic LM1 and BM1 enhance tumorigenesis and metastasis potential

LM1 and BM1 were injected into mouse DP according the cell number in (A). Tumor rate were summarized. Tumor latency is shown in the parentheses. (B-C) Metastasis profiles and image of LM1 and BM1 generated tumors. 
line (Patrawala et al., 2005). Strikingly, we found that the SP was significantly increased in BM1 cells in both PC3 and DU145 (Figure 4.22). It suggests that SP were enriched in these recovered BM1 cells. Additionally, both CXCR4 and CD24, which were reported to be linked with both CSCs and metastasis, indeed were highly up-regulated in both LM1 and BM1 compared with their parental PC3 bulk cells (Figure 4.21). CXCR4 expression increased from 8.25\% (bulk PC3 cells) to $14.1 \%$ ( two-fold in LM1) and 38.8\% (4.5-fold in BM1) respectively. CD24 expression is increased from $21 \%$ (bulk PC3 cells) to $47.3 \%$ (two-fold in LM1) and $67.1 \%$ ( three-fold in BM1). It is very interesting to note that BM1 seems to be more enriched in CSC properties. Previous studies indicated that stem cell markers are overexpressed in distant metastatic organs (Balic et al., 2006); however they did not perform functional assays by isolating these metastatic cells to do animal experiments. We not only checked expressions level of these markers, but also tested the CSC properties by in vivo experiments. Therefore, after we indicated that primary tumors hold CSC features, we subsequently proved that these distant metastatic cells are enriched in CSC properties. It demonstrates that CSC properties accompany tumor metastasis continually and correlate with metastasis progression. These stemness genes are equal to metastasis properties. This again implies that CSC is the population which drives metastasis. It is still very interesting to study how these CSC properties are enriched in these distant metastatic cells. Additionally, the SP was evident in our BM1 line, because SP is one feature of bone marrow cells (Lin and Goodell, 2006) and BM1 is derived from bone marrow metastasis; it is possible that there was fusion between these invading cancer cells and resident bone marrow cells (Rizvi et al., 2006; Wang, 2010). Then cancer cells endowed these BM cells with additional properties (Wang, 2010) 


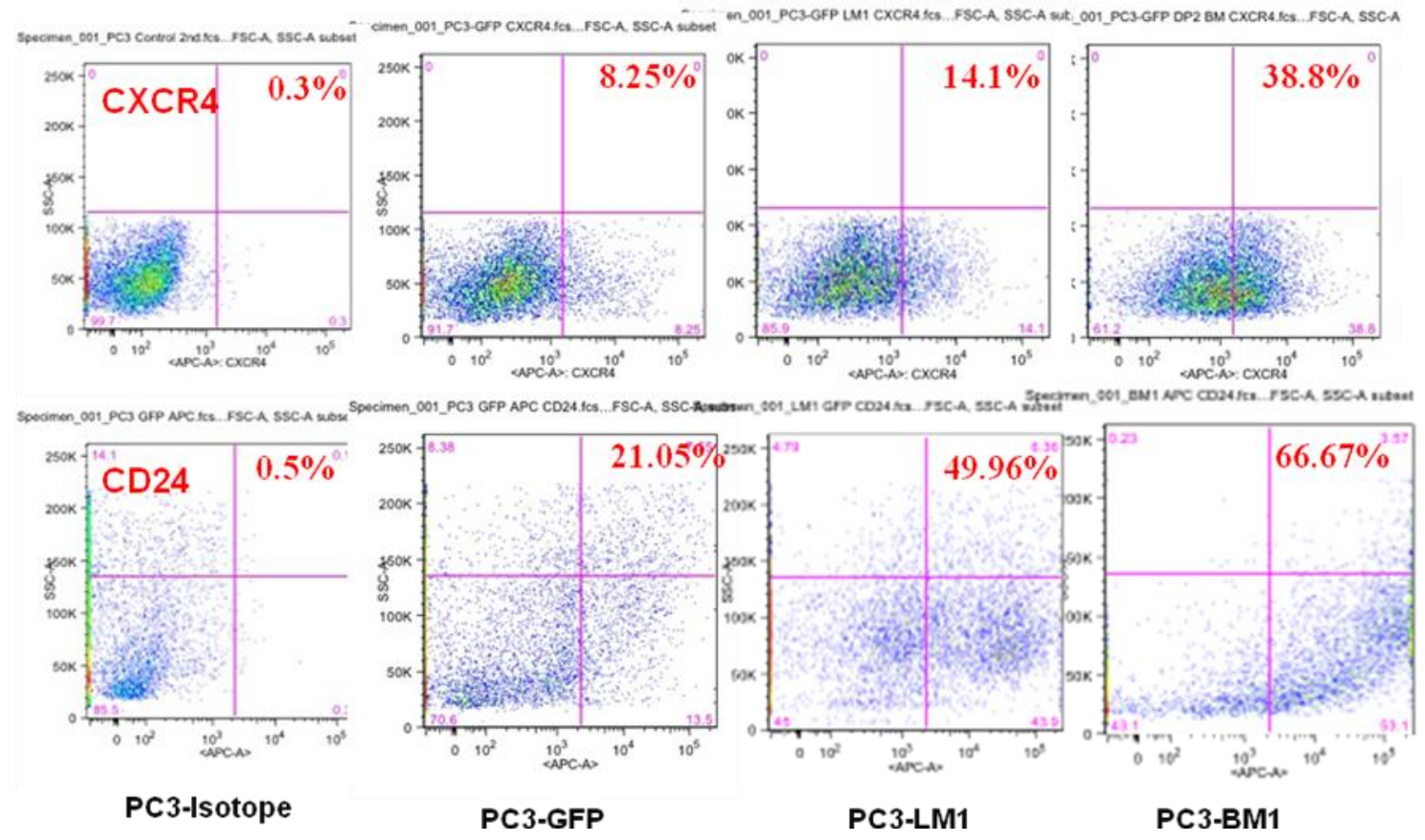

Figure 4.21 CD24 and CXCR4 expression are increased in LM1 and BM1

Both of CD24 and CXCR4 expression are up-regulated in PC3-LM1 and PC3-BM1 cell lines. 


\section{Side population were detected from the BM metastatic cells}

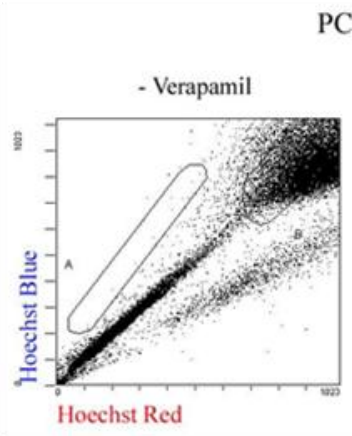

C3-GFP

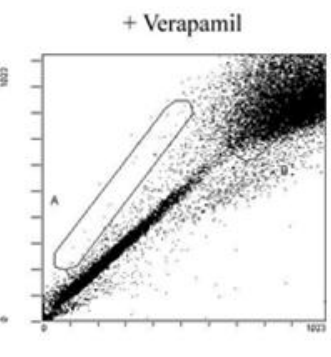

Hu PC3-GFP Ms Tumor Bone Metastases
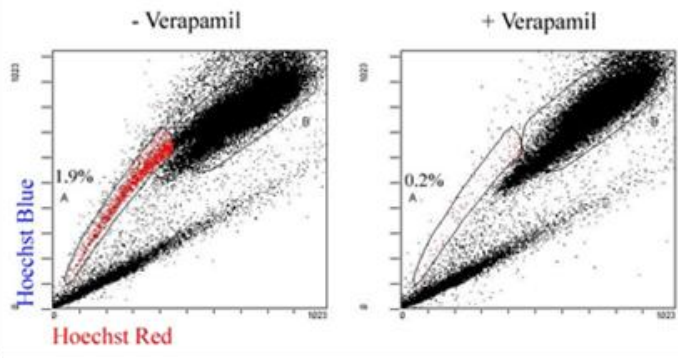

DU145-GFP
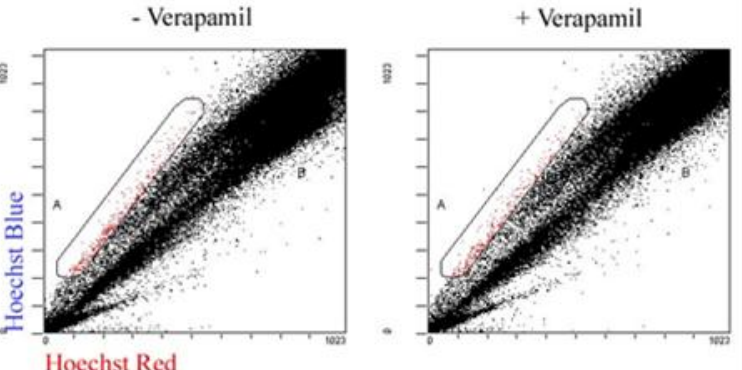

DU145-GFP BM

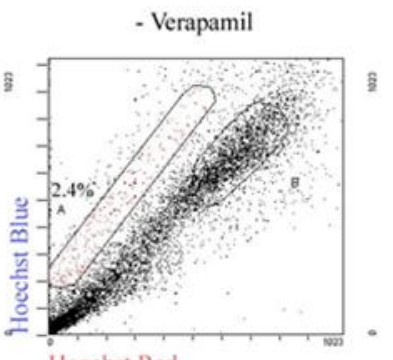

+ Verapamil

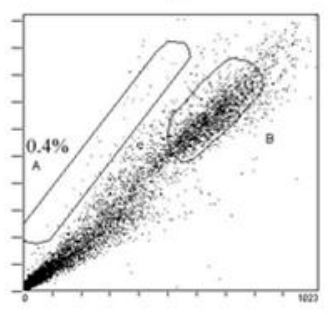

Figure 4.22 SP enriched in BM1 cells

BM1 cell lines derived from PC3 or DU145 metastasis demonstrate significantly larger SP phenotype. 
If this hypothesis is true, it may provide perfect evidence to support the cell fusion in cancer metastasis and a novel point for origin of cancer metastasis.

\section{TGF- $\beta 2$ Is A Key Regulator Which Modulates Metastasis, Tumor Microenvironment, and CSC Properties}

The TGF- $\beta$ pathway plays a dual role in tumorigenesis and metastasis: It may act as a tumor suppressor in the early stages of tumor growth, then in the later stages, it may promote tumor development and metastasis (Padua and Massague, 2009). In prostate cancer, the TGF- $\beta$ pathway has been reported as both a tumor suppressor and an oncogene (Basanta et al., 2009). In our previous microarray, we found that TGF $\beta 2$ is up regulated in DP tumors. In the mouse array, we also found that one of the TGF pathway receptors, tgfbr1, is up-regulated in mouse stroma. On the other hand, multiple studies have shown that TGF- $\beta$ is also involved in regulation of stem cell pathways. More interestingly, TGF- $\beta$ is also one of the key regulators of the tumor microenvironment. These pieces of evidence suggest that TGF- $\beta$ might be a key regulator of metastasis by modulating CSC pathways and interactions with the surrounding microenvironment. In order to verify the role of the TGF- $\beta$ pathway in our mouse DP prostate metastasis model, we used GIPZ shRNA to knock down TGF- $\beta 1$ and $\beta 2$ respectively and produced two stable PC3 lines with TGF- $\beta 1$ and $\beta 2$ knockdown (figure 4.23). By injecting these cells into mouse DP, we observed different effects on metastasis suppression. First, when we injected 500,000 cells in each group, the tumor rates were the same as all the injections produced tumors. Tumor weight was not significantly different among these three groups. 

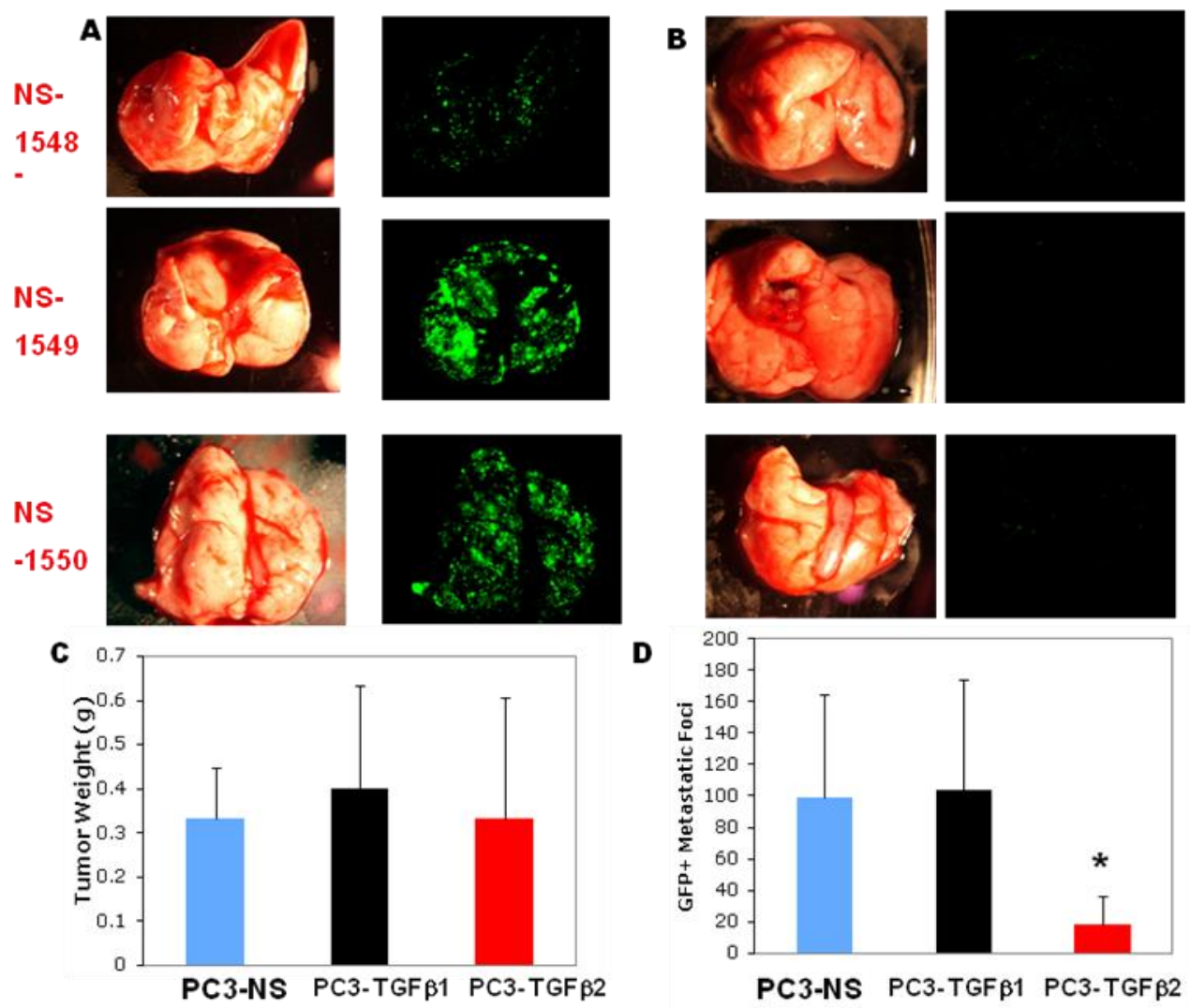

TGFB2-714
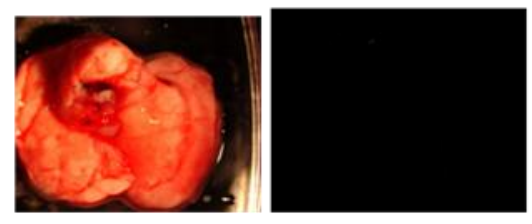

TGFB2-715

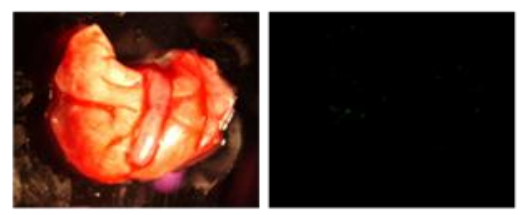

TGFB2-716

D

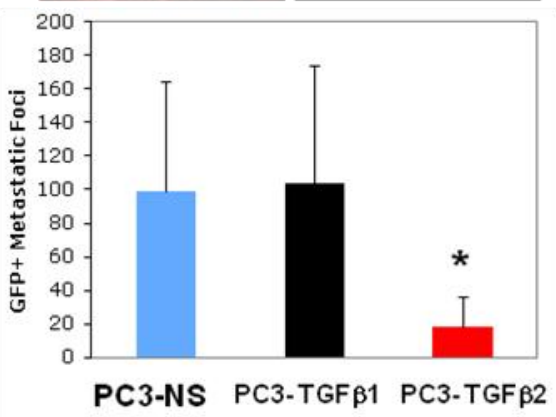

\section{Figure 4.23 PC3-GIPZ-TGFß2 KnockDown suppressed metastasis}

TGF 32 knockdown inhibits PC3 cell metastasis. .PC3 cells were infected with either pGIPZ-NS or pGIPZ-TGF $\beta 2$-shRNA lentiviral vectors (at MOI 25) $48 \mathrm{~h}$ later, 500,000 cells of each type were injected in 50\% Matrigel into the DP of NOD/SCID mice. Animals were terminated 40 days after implantation. Representative lung Images were shown in A (PC3-NS) and B (PC3 TGF 32,3 animals each). C.D. show the tumor weight and lung metastasis of PC3 NS, TGF $\beta 1$, TGF $\beta 2$ group. TGF $\beta 1$ did not show the inhibitory effect on metastasis. . 
However, metastases were sharply reduced in the TGF- $\beta 2$ GIPZ group as assessed by checking the lung metastasis compared with TGF- $\beta 1$ and NS control group. The TGF- $\beta 1$ GIPZ group did not show any inhibitory effect on metastasis compared with the NS control. This further confirmed our microarray data since TGF- $\beta 1$ expression did not show any change between DP and sc tumors. Our animal data also revealed that TGF- $\beta 2$, instead of TGF- $\beta 1$, might play a critical role in regulating prostate cancer metastasis.

After we identified that TGF- $\beta 2$ actually could suppress tumor metastasis, it became interesting to explore if these CSC markers are among the down-stream targets of the TGF- $\beta 2$ pathway. CD44 is interesting in this pathway since previous studies have shown that TGF- $\beta$ regulates CD44 in CSCs (Godar et al., 2008). More interestingly, TGF- $\beta$ was reported to directly regulate OPN and both OPN and TGF- $\beta 2$ are involved in regulating the tumor microenvironment. The relationship between OPN and TGF- $\beta 2$ in prostate cancer is still unclear. In order to investigate the mechanism of TGF- $\beta 2$ regulation of metastasis, CSCs and tumor microenvironment, we first checked the OPN expression by using PC3-GIPZ-TGF- $\beta 2$ tumor and cell lines (Figure 4.24A). OPN expression was reduced both in IHC and flow cytometry analysis in PC3-GIPZ-TGF- $\beta 2$ tumor and stable cell lines. It suggests that OPN is down-stream of the TGF- $\beta 2$ pathway. We performed Western blotting for CD44 expression in the TGF- $\beta 2$ knockdown stable cell line and found that CD44 significantly decreased with TGF$\beta 2$ knockdown (Figure 4.24B). It is interesting that CD44 is the one of the receptors of OPN (Weber et al., 1996). We already showed knockdown OPN also decreased the expression of CD44 (data not shown). It might be possible that OPN, CD44 and TGF- $\beta 2$ are in the same signal transduction pathway. Furthermore, MMP9 was reported as one target of the TGF- $\beta 2$ 
A
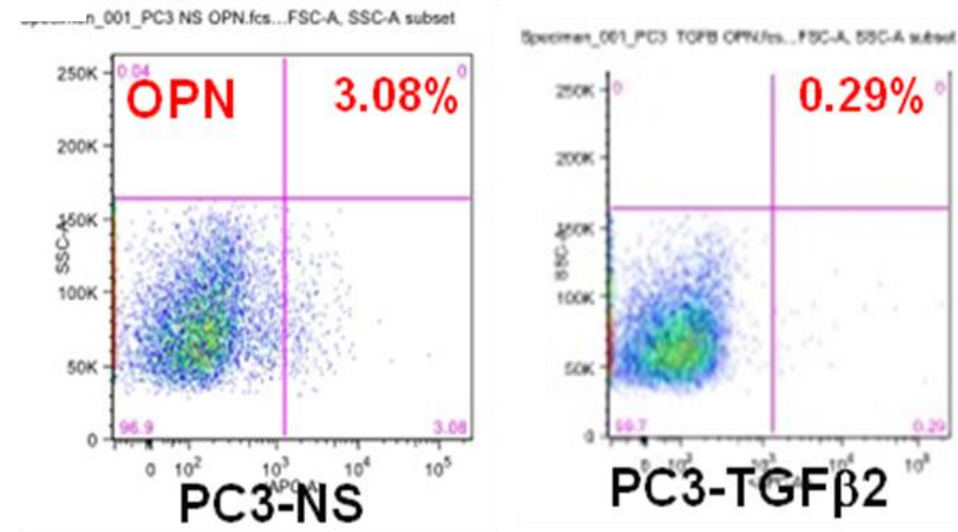

B

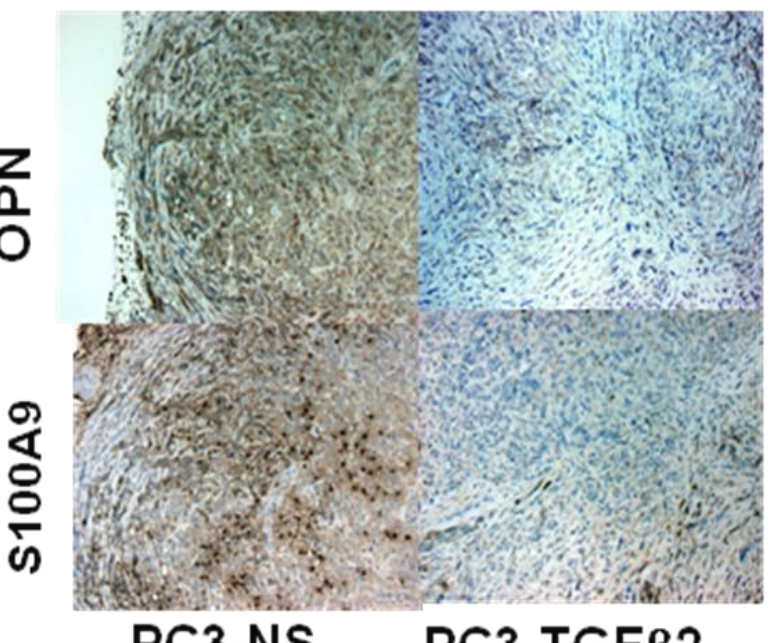

PC3-NS PC3-TGF $\beta 2$

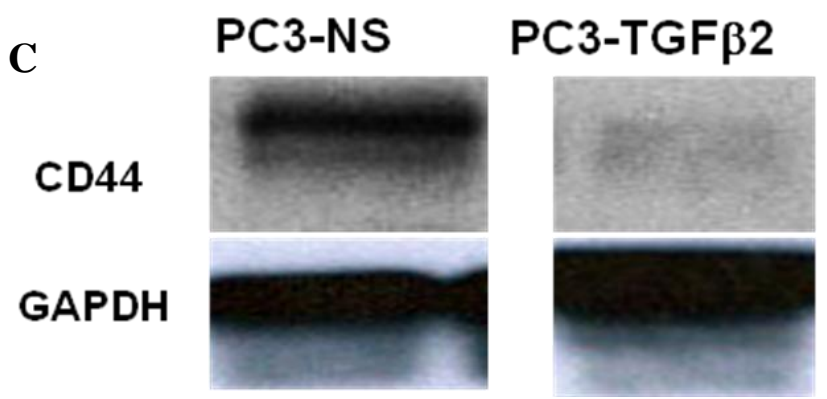

Figure 4.24 TGF- $\beta 2$ regulated stem cells markers OPN and CD44 expression

TGF 32 knockdown suppress the OPN expression in PC3- TGF 32 stable lines. (B) IHC demonstrate the PC3TGF 32 xenograft tumor also decreased expression of OPN and S100A9. (C) TGF 32 knockdown suppress the CD44 expression in PC3- TGF $\beta 2$ stable lines 


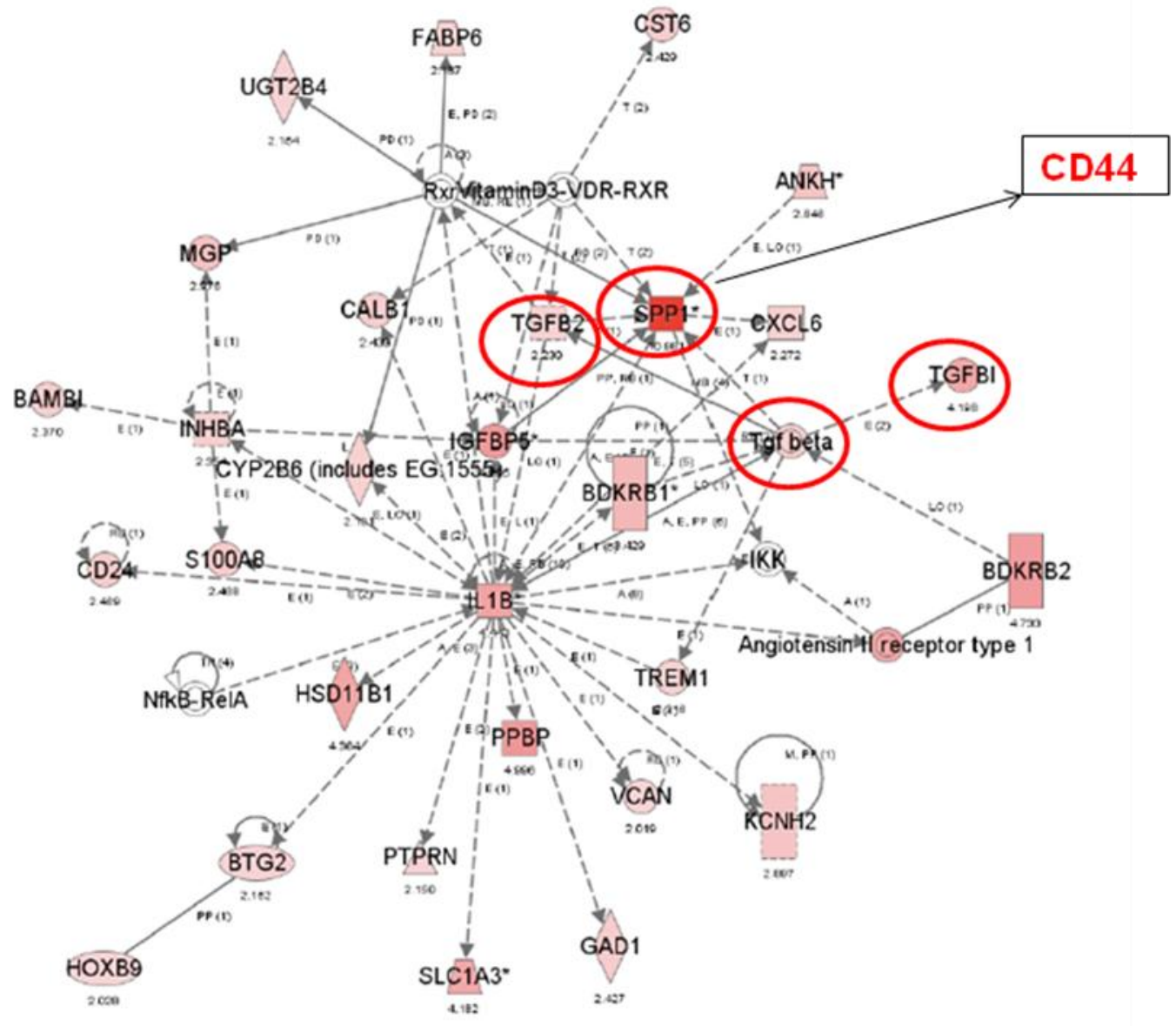

Figure 4.25. Ingenuity Pathway Analysis (IPA) of Up-regulated genes in DP vs sc comparisons (human).

Pathway analysis based on the Ingenuity Pathway Knowledge Base (IPKB). The two highest scoring networks (score 46, 43; a score 3 or greater is considered significant ( $\mathrm{p}$ < 0.001) associated to cancer, cellular movement, Cell growth and proliferation were combined. Intensity of the node color (red) represents the level of fold change. The shape of the node indicates the major function of the protein. A line denotes binding of the products of the two genes while a line with an arrow denotes 'acts on'. A thick line denotes direct interaction and a dotted line denotes an indirect interaction and the orange lines indicate potential interaction. 
Pathway Chou et al, 2006). More importantly, using the TGF- $\beta 2$ knockdown line, we also found that the mouse S100A9 was significantly decreased. This suggests that TGF- $\beta 2$ is involved in regulating these interactions between cancer cells and host tissue (figure 4.29.D). We also further perform the Ingenuity Pathway Analysis (IPA) to analysis the up-regulated genes in DP vs sc comparisons (human) (Figure 4.25). TGF- $\beta 2$ pathway is still in the two highest scoring networks (score 46, 43; a score 3 or greater is considered significant (p < 0.001) associated to cancer, cellular movement, Cell growth and proliferation were combined). And IPA suggested TGF- $\beta 2$ pathway connected with OPN and S100A9 pathway. Overall, these pieces of evidences illustrate that TGF- $\beta 2$ indeed plays a critical role in regulation of metastasis and might modulate metastasis through CSC pathways by regulating two important stem cell markers, OPN and CD44. These results provide further support for our hypothesis that TGF- $\beta 2$ may be the master regulator of tumor metastasis through modulating CSCs.

\section{Host Microenvironment Genes Identified Through Mouse-gene Microarray Analysis}

As a significant number of inflammation/immune-related genes $(\sim 8 \%)$ are upregulated in the human gene microarray in DP tumors, it implies that the host immune system may be involved into this phenomenon. We wondered whether or not we could obtain a similar microarray gene signature that may be akin to our human array signature, in order to draw a whole picture for demonstrating how the host microenvironment contributes to this highly metastatic microenvironment. To gain insights into this question, we carried out microarray analysis by using the same samples used in our human array with the Agilent's 44K mouse whole genome oligo microarray, which detects only mouse-specific genes. The microarray experiments were 
performed exactly as the human arrays. The results indicate that this dramatic gene signature does not only exist in our human array, but also is found in the mouse genomic microarray. The mouse array turns out to have many of the same trends found in our human array. Very strikingly, a significant number of mouse myo-fibroblast and myo-epithelial genes are up regulated in the mouse DP microenvironment (table 4.4). It suggests that the DP microenvironment may be the pre-metastatic and pre-CSC niche due to these highly reactive stroma. Reactive stroma has been reported to correlate with prostate cancer progression and metastasis (Anborgh et al., 2010; Dakhova et al., 2009). To verify this hypothesis, we analyzed one of the myo-fibroblast markers, $\alpha$-SMA, in mouse DP and sc tumor by IHC (Figure 4.29 A). As the results show, mouse $\alpha$-SMA significantly increased in both LAPC9 and PC3 DP tumors compared with sc tumors. This result indicates that there is indeed a reactive stromal microenvironment existing in the highly metastatic DP microenvironment.

There are in total 88 genes were found commonly increased in both the human and the mouse array (Figure 4.26). Strikingly, most of these commonly up-regulated genes are either metastatic genes related, such as Matrix metallopeptidase9 (MMP9), hepatocyte growth factor (HGF), and SPARC, or, of great significance and interest, multiple development/stem cellrelated genes including BMP3, BMPR1B, WNT4, HAS2, PROM1, HOXD13, ABCG2, SOX9, SOX11, and aldehyde dehydrogenase (ALDH). This unique phenomenon strongly suggests that development/ stem cell pathways regulate tumor metastasis and tumor microenvironment. Our result is correlated to a recent study in primary human prostate sample (Dakhova et al., 2009). These common up-regulated genes in both human and mouse tissue are not due to inefficient probes that could not distinguish well between human and mouse genes. Several 


\section{Table 4.4. Representative mouse genes commonly upregulated in $3 \mathrm{DP} / \mathrm{SC}$ tumor pairs}

\begin{tabular}{|c|c|c|c|c|}
\hline Probe name & Gene name & Systematic nam & Description & Fold increase \\
\hline \multicolumn{5}{|c|}{ Mouse prostate and related genes } \\
\hline P191669 & Chgb & NM007694 & Mus musculus chromogranin B (Chgb) & $23.3-37.7(10 x)$ \\
\hline P461822 & Sv p2 & NM009300 & Mus musculus seminal vesicle protein 2 & 23.7 \\
\hline P353162 & Sv s5 & NM009301 & Mus musculus seminal vesicle secretion 5 & 15.2 \\
\hline P194293 & Sv s 7 & NM020264 & Mus musculus seminal vesicle protein, secretion 7 & 5.7 \\
\hline P397983 & Slc9a2 & AK077026 & Mus musculus adult male testis cDNA & $9.8,4.5$ \\
\hline \multicolumn{5}{|c|}{ Inflammation/immune functions } \\
\hline P353252 & Mal2 & NM178920 & Mus musculus mal, T-cell differentiation protein 2 & 10.5 \\
\hline P399305 & Tnfrsf19 & NM013869 & Mus musculus TNFR superfamily, member 19 & 5.6 \\
\hline \multicolumn{5}{|c|}{ Cell survival/apoptosis } \\
\hline P352266 & Bcl-2 & NM000633 & anti-apoptotic protein & $2.7-3.8(10 x)$ \\
\hline \multicolumn{5}{|c|}{ Extracellular matrix/cytoskeleton/motility/proteases } \\
\hline P416858 & Myl1 & NM021285 & Mus musculus myosin, light polypeptide 1 & 11.7 \\
\hline P338072 & Myh4 & NM010855 & Mus musculus myosin, heavy polypeptide 4 & $11.2,6.8$ \\
\hline P143296 & Myh8 & NM177369 & Mus musculus myosin, heavy polypeptide 8 & 9.6 \\
\hline P552036 & Myh2 & NM001039545 & Mus musculus myosin, heavy polypeptide 2 & $6.7,4.6$ \\
\hline P455157 & Mybpc2 & NM146189 & Mus musculus myosin binding protein $\mathrm{C}$ & 6.4 \\
\hline P192042 & Myh1 & NM030679 & Mus musculus myosin, heavy polypeptide 1 & 5.8 \\
\hline P383572 & Mylpf & NM016754 & Mus musculus myosin light chain, phosphorylatable & 5.4 \\
\hline P488386 & Myot & NM001033621 & Mus musculus myotilin & 4.2 \\
\hline P622418 & Tnnt3 & NM011620 & Mus musculus troponin T3 & 7.0 \\
\hline P353232 & Tnnc2 & NM009394 & Mus musculus troponin $\mathrm{C} 2$, fast & 4.1 \\
\hline P656699 & Actn3 & NM013456 & Mus musculus actinin alpha 3 & 5.1 \\
\hline P107020 & Kif5a & NM001039000 & Mus musculus kinesin family member $5 \mathrm{~A}$ & 4.5 \\
\hline P516409 & Col4a6 & ВC004800 & Mus musculus procollagen, type IV, alpha 6 & 7.8 \\
\hline P302544 & $\mathrm{Col} 8 \mathrm{a} 2$ & NM199473 & Mus musculus procollagen, type VIII, alpha 2 & 5.8 \\
\hline P381484 & Spoon2 & NM133093 & Mus musculus spondin 2, ECM protein & 6.2 \\
\hline P64356 & Sparcl1 & NM010097 & Mus musculus SPARC-like 1 (mast9, hevin) & $5.6,5.1$ \\
\hline \multirow[t]{3}{*}{ P408728 } & Plp1 & NM011123 & Mus musculus proteolipid protein (myelin) 1 & $5.3,5,5,5,4.7,4.6$ \\
\hline & & & & $4.6,4.6,4.5,4.5$ \\
\hline & & & & 4.2 \\
\hline P426096 & Mmp7 & NM010810 & Mus musculus matrix metallopeptidase 7 & 4.1 \\
\hline P18116 & $\mathrm{Ccl} 24$ & NM019577 & Mus musculus chemokine (C-C motif) ligand 24 & 4.0 \\
\hline P507214 & Mmp9 & NM013599 & Mus musculus matrix metallopeptidase 9 & 3.84 \\
\hline \multicolumn{5}{|c|}{ Developmental pathways/stem cell-related } \\
\hline P248865 & Foxf2 & NM009846 & Mus musculus forkhead box F2 & 9.4 \\
\hline P244193 & $\mathrm{Cd} 24 \mathrm{a}$ & NM009846 & Mus musculus CD24a antigen & 9.3 \\
\hline P286748 & Frzb & NM011356 & Mus musculus frizzled-related protein & 8.5 \\
\hline P278368 & Dner & NM152915 & Mus musculus delta/notch-like EGF-related receptor & $6.3,5.3$ \\
\hline \multirow[t]{2}{*}{ P214630 } & Sox9 & NM011448 & Mus musculus SRY-box containing gene 9 & $6.2,5 \cdot 6,5 \cdot 5,4 \cdot 6$ \\
\hline & & & & $5.1,4.6,4.4,4.1$ \\
\hline P54176 & Axin 2 & NM015732 & Mus musculus axin2 & 5.8 \\
\hline P457196 & Sfrp4 & NM016687 & Mus musculus secreted frizzled-related sequence & 5.8 \\
\hline P437421 & Bmper & $\mathrm{NM} 028472$ & Mus musculus BMP-binding endothelial regulator & 5.2 \\
\hline P61864 & Wnt2 & NM023653 & Mus $\mathrm{m}$. wingless-related MMTV integration site 2 & 5.2 \\
\hline P414072 & Proml & NM008935 & Mus musculus prominin 1 & 4.5 \\
\hline P586944 & Bmpr $1 b$ & NM007560 & Mus musculus BMP receptor, type $1 \mathrm{~B}$ & 4.0 \\
\hline $\mathrm{P} 466285$ & Hoxa10 & NM008263 & Mus musculus homeo box A10 & 3.98 \\
\hline
\end{tabular}

* A total of 593 genes representing 423 known genes and 170 unknown genes showed $\geqslant 2$ fold increases in mRNA levels. For some genes (such as SPP1) multiple probe sets (numbers indicated in parentheses by $\mathrm{x}$ ) were detected and the range of fold increases was given. Genes indicated in red are selected for the gene signature. 
reasons support this idea: In total 44,000 genes were probed in both human and mouse arrays, if the probe is not species-specific, one would expect to find more than 88 commonly upregulated genes. More importantly, all of these common genes are functional genes related to metastasis, inflammation, stem cells and reactive stroma. It is not likely for these categories to be chosen at random. Nevertheless, these common genes' presence implied that both human and mouse genes share some important common signaling pathways in metastasis, inflammation and stem cells. For example, there may be some stem cell stimuli such as cytokines that could stimulate both human and mouse components to increase stem cell gene expression level. This common upregulated gene list in both mouse and human systems give us more reason to believe that there is a stem cell pathway underlying this active microenvironment. Finally, it is obvious that a tumor and host paracrine/autocrine crosstalk exists between human tumor cells and mouse host cells. Cytokines such as IL-1 $\beta$, CCR1, FGF7, and TGF- $\beta 2$ were found to be significantly up-regulated in the human array in DP tumors; correspondingly, these cytokines' receptors, like Illr2, Ccrll, Fgf10, and tgfbr1, were highly increased in mouse arrays in the DP tumors (figure 4.27). In addition, we also observed an increase in macrophage marker S100A9 in DP tumors relative to sc tumors (figure $4.28 \mathrm{~B}$ ). Macrophages are major players in cancer-related inflammation and interactions between epithelial cells and host stromal cells (Sangaletti et al., 2008). The increased S100A9 indicates that the DP microenvironment induced metastasis may be dependent on macrophage regulated epithelial-stromal interaction pathways. Although we are not certain which murine cells in the DP tumors are responsible for these unique gene expression profiles, these results strongly 
Important signaling molecules commonly upregulated in both human and mouse arrays (in total: 88 )

\begin{tabular}{ll} 
HPCa cells & \multicolumn{1}{c}{$\begin{array}{c}\text { Mouse prostate ce } \\
\text { (stromal/mesenchy } \\
\text { inflammatory/epit }\end{array}$} \\
\cline { 2 - 3 } HGF & Hgf \\
SPARC & Sparc1 \\
MMP9 & Mmp9 \\
ADAM23 & Adam23 \\
ADAMTS6 & Adamts6 \\
EDNRA & Ednra \\
ALOX5AP & Alox5ap \\
PROM1 (CD133) & Prom1 \\
ABCG2 & Abcg2 \\
CD24 & Cd24a \\
ALDH1 & Aldh1 \\
HOXB9 & Hoxb9 \\
HOXD13 & Hoxd13 \\
WNT4 & Wnt4 \\
BMPR1B & Bmpr1b \\
BMP3 & Bmp3 \\
CHRD (Chordin) & Chrd12 \\
TNNT3 (troponin T3) & Tnnt3 \\
ACTA2 (smooth muscle actin, $\alpha 2)$ & Acta2 \\
MYH4 & Myh4 \\
FGG (fibrinogen, $y$ chain) & FGG \\
HAS2 (hyaluronan synthase 2) & Has2
\end{tabular}

Figure4.26 Commonly upregulated genes in both human and mouse 


\section{Tumor-host paracrine/autocrine cytokine signaling}

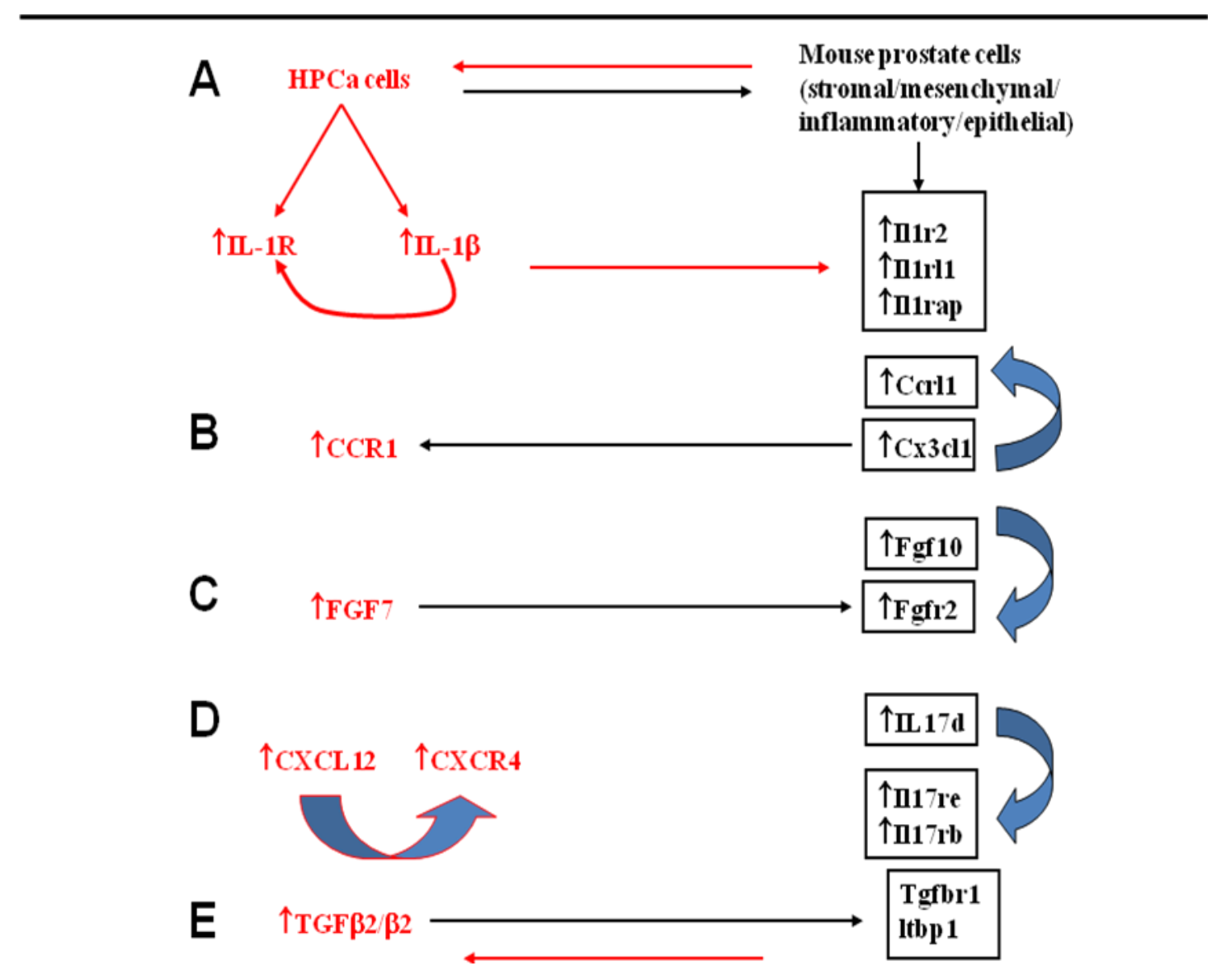

Figure 4.27 Tumor host cross talk pathway 


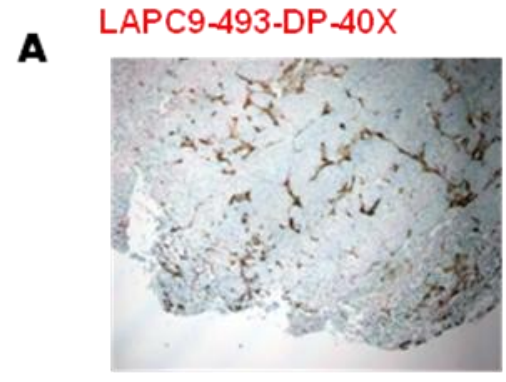

LAPC9-212-SQ-40X

B

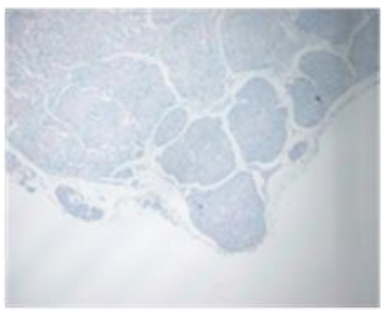

G

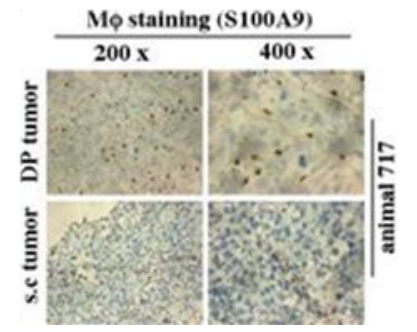

\section{C}

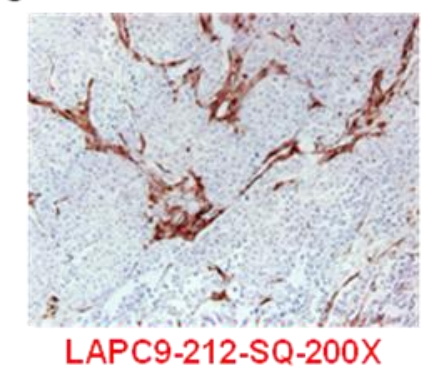

D
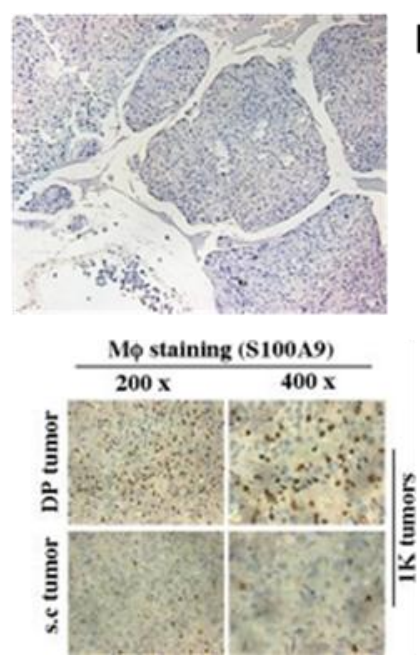

E PC3-1524-DP-40X

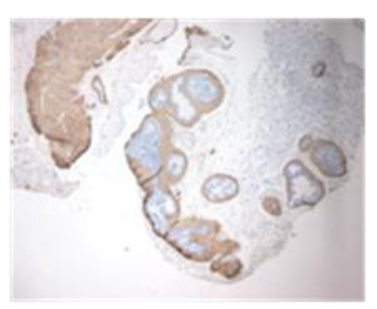

PC3-1520-SQ-40X

$\mathbf{F}$

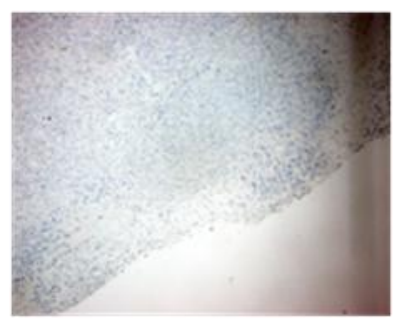

\section{Figure $4.28 \alpha$-SMA/S100A9 expressions in LAPC9 and PC3 DP vs s.c.}

IHC staining of $\alpha$-SMA in (A) LAPC9-DP tumor , 40x (B) LAPC9-sc tumor 40x; (C) LAPC9DP tumor, 200x; (D) LAPC9-sc tumor , 200x; (E) PC3-DP tumor , 40x; (F) PC3-sc tumor 40x.(G) Shown were representative images of IHC staining using a mAb to S100A9, a macrophage-specific molecule. Images from two tumors (200 x and 400x each) were shown. 
suggest that the host cells appear to be creating a pro-inflammatory and perhaps a stem cellenriched microenvironment for the implanted human $\mathrm{PCa}$ cells to promote a metastasis cascade.

All together, these human and mouse arrays suggest a very highly metastatic, inflammatory, and CSC-related tumor microenvironment existing in mouse orthotopic implantation sites. The specific microenvironment may function like a stem cell niche. It is enriched with fibroblasts and myoepithelial cells that form a physical anchor for stem cells to protect them from depletion. The specialized microenvironment also orients them to regulate stem-cell number, proliferation rate, and differentiation during environmental and genetic stimuli. The niche is unique in that some stem cells are not able to fully function without it. While the function of the niche is well studied in normal stem cells, there are still few studies which work on the cancer stem cell niche (Bissell and Labarge, 2005; Sneddon and Werb, 2007). Is there a

physically existing cancer stem cell niche which promotes cancer progression and metastasis? Some evidence indicates that the tumor microenvironment influence drug resistance and tumor dormancy (Vera-Ramirez et al., 2010). Because these are two important features of CSCs and metastasis, these data hint that the tumor microenvironment may regulate CSCs during metastasis. However, this is indirect evidence; our current research might be the first to direct answer this question. Targeting of CSC is fast developing in cancer therapy (Dubrovska et al., 2009), but most of these studies pay attention on these epithelial CSC, when the data suggests that we may be wise to target both epithelial and stroma.

\section{Reconstitution/mimicking of the Metastatic Microenvironment in Mouse Subcutis}


It is a very interesting question as to what would happen if we put metastatic cells into the subcutis, as it helps to answer the question as to what is more critical, the microenvironment or intrinsic properties, in deciding tumor metastasis. Since the DP microenvironment exhibits a highly-metastatic microenvironment, it is reasonable to attempt to rebuild this specific niche in subcutaneum. To test this idea, we implanted several types of cells into mouse subcutis and checked the metastasis development. We first used freshly isolated DU145 and LAPC9 DP tumor cells to re-inject into mouse sc. We did not see further metastases from these DP derived sc tumors. It implies that DP cells may lose their metastasis/stem cell potential under the influence of a sc microenvironment (data not shown). Since we hypothesized that CSCs could be the subpopulation which fuels metastasis, we further injected CD44 ${ }^{+}$and SP cells from LAPC9 into mouse sc; we still did not get dramatic metastasis from this experiment as we observed with DP injection (figure 4.29). Because we have identified OPN and CD24 as two putative metastatic CSC markers, we further tested $\mathrm{OPN}^{+}$and $\mathrm{CD} 24^{+}$populations by injecting them into the sc site. These experiments indicated that only $\mathrm{OPN}^{+}$cells produce much stronger metastasis than the negative subpopulation; surprisingly(figure $4.29 \mathrm{~A}), \mathrm{CD}_{2}{ }^{+}$cells did not develop metastasis in the sc site( data not shown). In addition, our previous experiment shows that distant metastatic cells are enriched in CSC features, as well as in metastatic ability. Then we injected BM1 into mouse sc, only a slight increase was shown in lung metastasis compared with parental cells (data not shown). When we injected another renal lymph node metastasis derived line ( RLM1) into subcutis, RLMI bearing mice show a significant increase of lung metastasis compared with parental cells ( figure $4.29 \mathrm{C}$ ). These results indicate that the metastatic limitations in mouse subcutis are sufficient to further prevent cancer with intrinsic 
A

$$
\mathrm{OPN}^{+} 100 \mathrm{~K}
$$

s.c. Lung
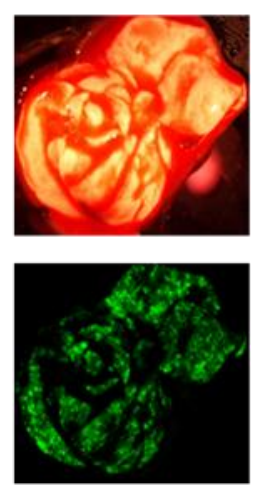

OPN-

100K s.c.Lung
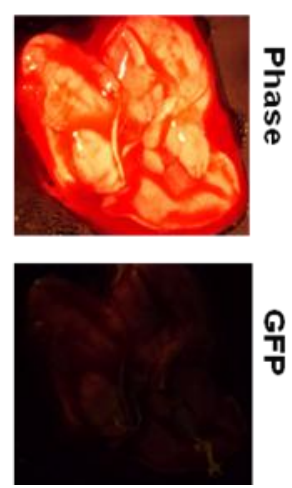

B

PC3-GFP-SQ-LUNG
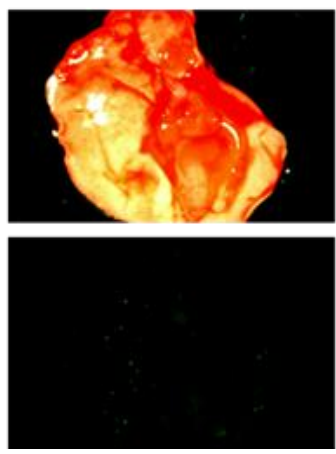

PC3-GFP-SQ+ hs5-LUNG
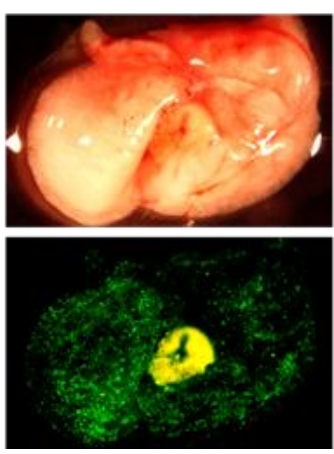

C

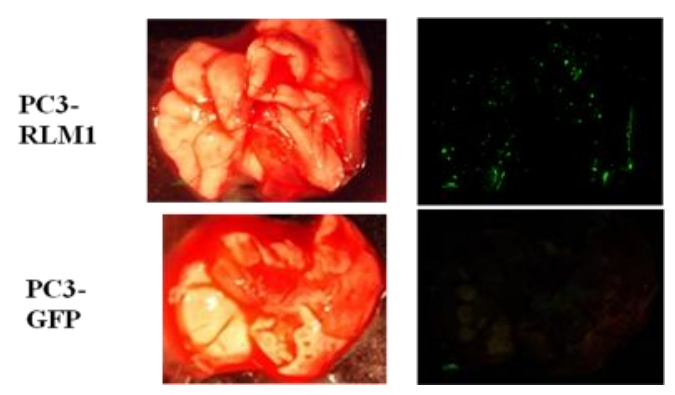

Figure 4.29 Reconstitute Metastasis profiles in mouse subcutis

(A) $100,000 \mathrm{OPN}^{+}$injected into subcutis and produce strong metastasis (B) 100,000 PC3-GFP cells co-injected with $1 \times 10^{6}$ Hs 5 cells into the subcutis, dramatically increased the metastasis. (C) Adrenal LN derived metastatic cells RLM1 were reinjected into mouse subcutis, which slightly increased the metastasis compared with PC3-GFP cells. 
CSC properties from spreading. It reflects the critical role of the tumor microenvironment in ultimately deciding cancer metastasis. There are some clinical relevance points in that we can potentially focus on remodeling the tumor microenvironment into a sc-like microenvironment to prevent or suppress tumor metastasis. However, the microenvironment is not absolutely limiting, both intrinsic cell properties and microenvironment play critical roles in regulating metastasis. For example, $\mathrm{OPN}^{+}$and RLM1 still can metastasize under sc. It suggests that these cell populations may have stronger intrinsic properties in metastasis so that it can overcome the metastatic limitation of sc microenvironment. Moreover, when Hs5 human mesenchemal stem cells (MSC) were co-injected with PC3 bulk cells into mouse sc, the bulk tumor cells develop dramatic lung metastasis (Figure 4.29 B). This is very interesting since a recent study indicated that MSC are able to promote tumor metastasis through TGF- $\beta$ pathway (Karnoub et al., 2007). There may be an underlying connection between TGF- $\beta$ and MSC in our DP model as well as in our enrichment model. Based on this finding, we will overexpress TGF- $\beta 2$, as well as use the whole minced DP, to add cancer cells to co-implant into mouse subcutis to see whether or not these will raise metastasis. All together, this evidence suggests both intrinsic properties and microenvironment play critical roles in regulating metastasis. Compared with other CSC markers, OPN might be the best metastatic CSC marker with which to purify these seeds of metastasis.

\section{DP-derived Metastatic Gene Signature Linked to Clinical Malignance.}

We further verified our metastatic gene signature in human prostate cancer clinical data sets. We first applied meta analysis by matching our gene signature with nine clinical prostate cancer data 
$\mathbf{A}$

\begin{tabular}{lrr}
\hline DataSet & Primary Site & Metastatic \\
\hline Holzbeierlein_Prostate & 40 & 9 \\
Lapointe_Prostate & 62 & 9 \\
LaTulippe_Prostate & 23 & 9 \\
Magee_Prostate & 8 & 3 \\
Ramaswamy_Multi_cancer_2 & 10 & 3 \\
Ramaswamy_Multi_cancer & 10 & 3 \\
Vanaja_Prostate & 27 & 5 \\
Varambally_Prostate & 7 & 6 \\
Yu_Prostate & 64 & 24 \\
\hline
\end{tabular}

B

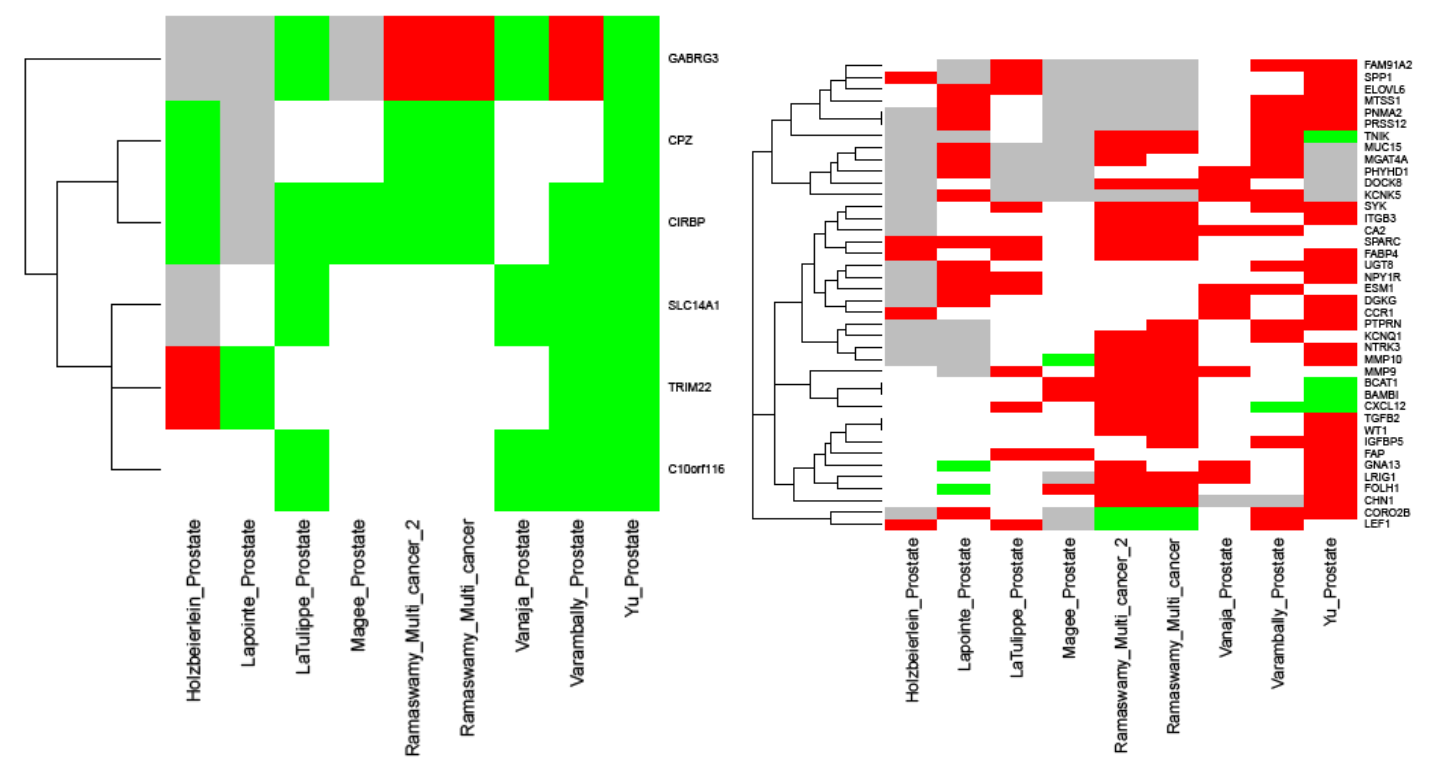

Figure 4.30 Genes whose down/up-regulated expression in metastasis validated by 9 data sets

Table for nine data sets (A), genes down regulated expression in these data sets (B), and (C) genes up-regulated expression in these data sets. 
sets. These nine data sets include primary tumor samples and metastasis samples, as well as clinical outcome ( figure 4.30 A). The principle we utilized for meta analysis is that if a gene is identified in more than three different data sets among these nine data sets, we count this gene as one involved in a gene signature. Under this principle, we found about 40 genes up -regulated from our gene signature in these clinical data sets including OPN, MMP9, TGF $\beta$ 2, CXCR12 (Figure 4.30C). It is very interesting that several of these genes were validated by our shRNA knockdown experiments including OPN and TGF- $\beta 2$ (Figure 4.10B).

We further adapted an unsupervised algorithm called consensus $\mathrm{k}$ means $(\mathrm{k}=2)$ clustering (Monti et al., 2003) to predict the prognosis of prostate cancer sample based three gene signatures. These three signatures are six down-regulated (Figure 4.31A and B) and 40 upregulated genes (Figure 4.31C and 1D) identified in above meta-analysis (figure 4.30), as well as the stem cell markers (Figure 4.31E, F) selected from our previous microarray.

The 1000 times consensus $\mathrm{k}$ means $(\mathrm{k}=2)$ clustering was performed on expression profile of 150 prostate cancer samples (131 primaries and 19 metatstases) available at GEO (GSE21034, PMID: 20579941). Table 1 shows the number of samples that were predicted into high and low risk, each group's overlap with metastasis samples and the survival analysis result based the BCR (Biochemical Recurrence) time.

Table 4.5 and figure 4.30 demonstrate that all three signatures can reveal distinct subgroups with substantial differences in time to biochemical (PSA) relapse (BCR). In this dataset, the six gene signature identified from meta analysis pop up as best signature. Based on six gene signature, all the 19 metastasis prostate cancer samples are predicted as high risk. In addition, the 
Table 4.5. Summary of prediction result based on three signatures

\begin{tabular}{lllr}
\hline & High Risk & Low Risk & SurvivalAnalysis \\
\hline Down & $63(100 \%)$ & $87(0 \%)$ & $2.97 \mathrm{E}-05$ \\
Up & $64(58 \%)$ & $86(42 \%)$ & 0.011 \\
StemCell & $92(95 \%)$ & $58(5 \%)$ & 0.007 \\
\hline
\end{tabular}

Note: Percentages in the parentheses denote the fraction of metastasis samples in each predicted class.

Down: the six down-regulated signature identified in my meta-analysis

Up: the 40 up-regulated signature identified in my meta-analysis

Stem cell: stem cell markers were selected from our human DP upregulated genes. 
A.

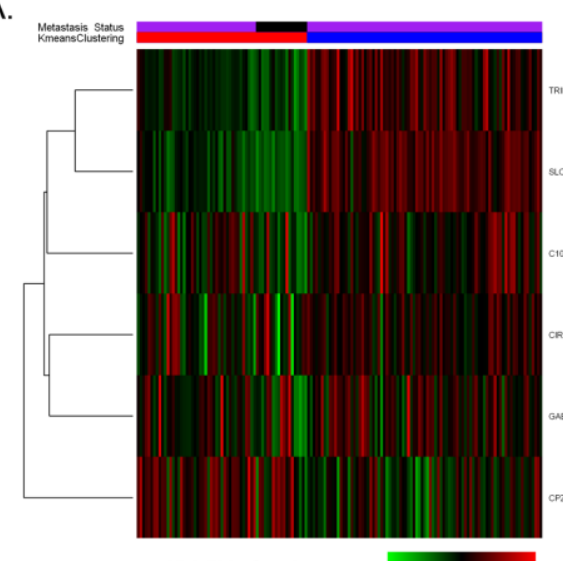

- High Risky Samples $\quad$ z score-4 0 Low Risky Samples

C.

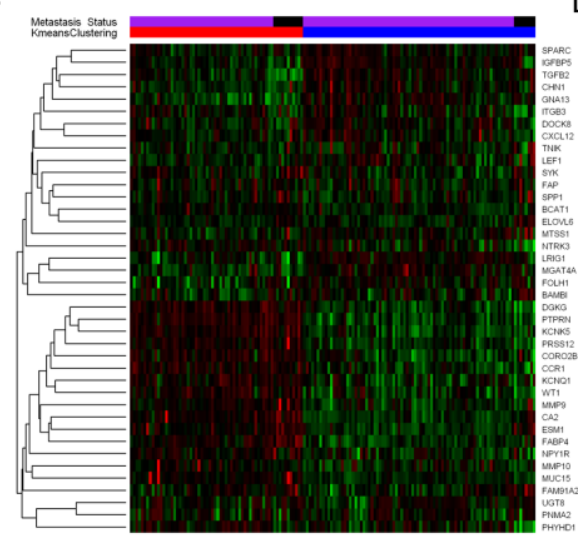

B.

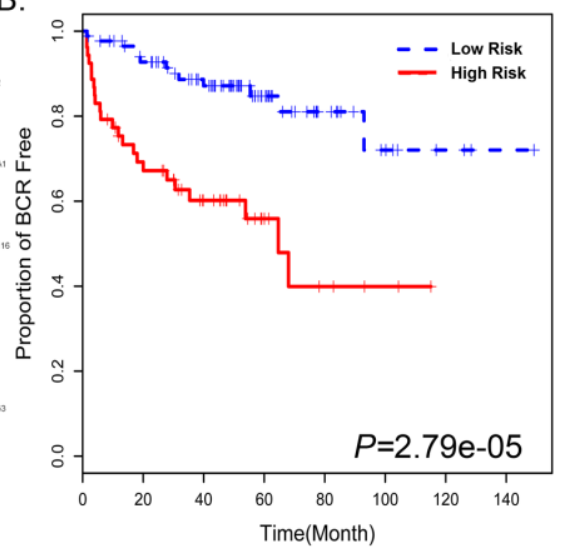

D.

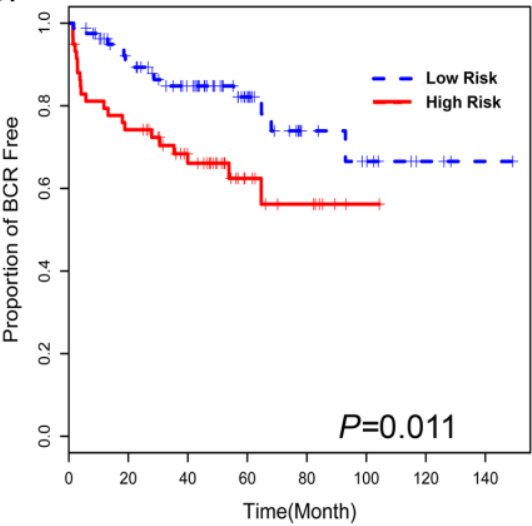

E.

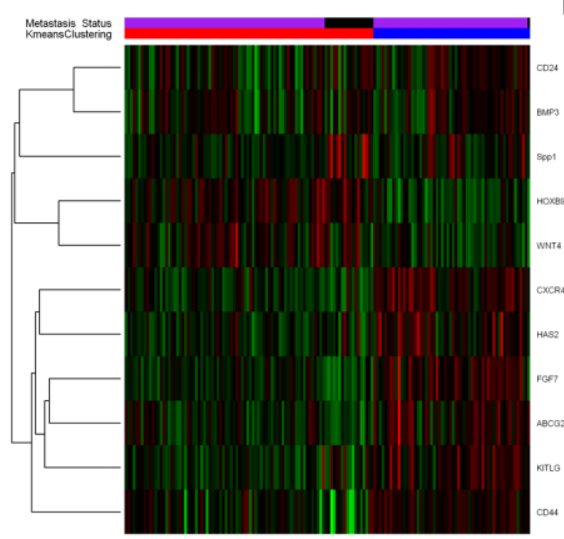

F.

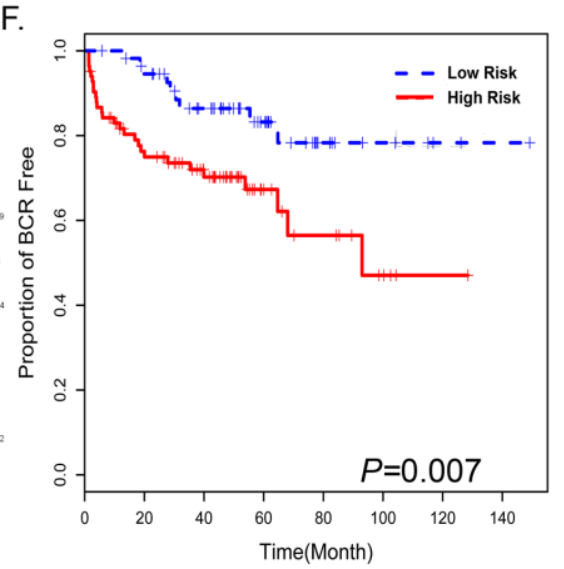

Figure 4.31. K-mean clustering identify high risk and low risk prostate cancer samples based on six down-regulated ( $\mathrm{A}$ and $\mathrm{B}), 40$ up-regulated genes ( $C$ and $D$ ) and the stem cell markers ( $E$ and F) 
difference of time to $\mathrm{BCR}$ is most prominent $(\mathrm{P}=2.97 \mathrm{E}-05$, figure $4.30 \mathrm{~B})$ among the three signatures. Based on the stem cell signature, 18 out of 19 metastasis prostate cancer samples are predicted as high risk. The stem cell signature also identified subgroups with distinct risk for $\mathrm{BCR}(\mathrm{P}=0.007$, figure $4.31 \mathrm{D})$. In contrast to six gene signature and stem cell signature, the 40 up-regulate signature shows no discriminative ability on metastasis prediction. However, the high risk and low risk samples characterized by this signature still demonstrated significant differences in time to $\mathrm{BCR}(\mathrm{P}=0.011$, figure $4.31 \mathrm{~F})$.

Figure 4.30A shows that six gene signature's expression, except CPZ, is coordinately downregulated in the high risk samples (especially in 19 metastasis prostate cancer samples), which is quite consistent to our microarray analysis and meta-analysis. For stem cell signature, the situation is a little bit complicated, we can see OPN, HOXB9 and WNT4 are unregulated in mestastasis samples and some other high risk samples. But not all the stem cell markers show consistently upregulation, we still need further to screen the best CSC markers which are correlated to clinical outcome.

It is exciting that our work from xenograft model are so closely to microarray, it suggest that these gene signature, and the CSC pathway might be the real in HPCa and involved in HPCa development and metastasis.

In the past decade, CSCs have been increasingly studied and CSC-based cancer therapy has been developed in mouse models. However, until recently, most CSC studies focused on primary tumor growth. The metastatic properties of CSCs and the " niche " of CSCs have been largely ignored. Studies on both CSCs' niche and metastatic CSCs are still in their infancy. There is no clear evidence to show the physical existence of a CSC niche in solid tumors. Our 
current study might provide an interesting model to show a highly metastatic, inflammatory, myofibroblast/myoepithelial -enriched, and stem cell-enriched tumor microenvironment as a CSC metastatic niche (figure30). Additionally, though researchers show evidence that $\mathrm{CD} 133^{+} / \mathrm{CXCR}^{+}$is the metastatic CSC population while $\mathrm{CD} 133^{+}$is only responsible for tumor initiation (Hermann et al., 2007), the more critical question is "What is the relationship between CSCs and metastatic CSCs"; This is still unanswered. In our current study, we provide a putative model for addressing metastatic CSCs. In our model, we believe there may be two types of metastatic CSCs: One may be the more potent CSCs, as they not only initiate tumor growth, but also spread metastases, e.g. $\mathrm{OPN}^{+}$subpopulation and LM1/BM1. The other type of metastatic CSC is a subpopulation of cells that is specifically functional in regulating metastasis but does not greatly influence tumor initiating. This subset might be a subtype of real CSCs. In this case, metastatic CSCs are a metastatic subtype of CSCs, while CSCs may contain other subtypes of cell population that can initiate tumor growth but not metastasis. For example, the $\mathrm{CD} 24^{+}$population is critical for metastasis, but may not influence tumor growth. In our DP /sc model system, the DP is the putative metastatic CSC niche, which may enriched/incubate both type of metastatic CSCs. Thus DP tumors, as well as these distant metastatic LM1/BM1 cells, are highly metastatic. s.c. tumors may lack CSCs or metastatic CSCs due to limitations imposed by the tumor microenvironment. The high tumor growth ability may be due to the tumor progenitor cells in s.c. tumors.

In summary, in our current research, we provide evidence to support the existence of a metastatic CSC niche. And this metastatic CSC niche (tumor microenvironment) is able to promote metastasis through regulating CSC phenotypes. Additionally, we identified the OPN ${ }^{+}$ 
subpopulation, as well as the $\mathrm{CD} 24^{+}$subpopulation as the metastatic CSCs. This evidence for metastatic CSCs will help us understand the underlying mechanisms of interaction between CSCs and the microenvironment. Nevertheless, we propose that TGF- $\beta 2$ may be a key regulator which modulates CSCs, microenvironment, and metastasis, and may help shape a highly metastatic niche. Our current study also indicates that both the tumor microenvironment and cell intrinsic properties are critical to tumor metastasis. Therefore, it is very important to target both of these two sides in clinical applications to eliminate primary cancer and metastasis. 


\section{References}

Abate-Shen C, Shen MM (2000). Molecular genetics of prostate cancer. Genes Dev 14: 2410-34.

Adams JM, Strasser A (2008). Is tumor growth sustained by rare cancer stem cells or dominant clones? Cancer Res 68: 4018-21.

Al-Hajj M, Wicha MS, Benito-Hernandez A, Morrison SJ, Clarke MF (2003). Prospective identification of tumorigenic breast cancer cells. Proc Natl Acad Sci U S A 100: 3983-8.

Albini A, Iwamoto Y, Kleinman HK, Martin GR, Aaronson SA, Kozlowski JM et al (1987). A rapid in vitro assay for quantitating the invasive potential of tumor cells. Cancer Res 47: 3239-45.

Allinen M, Beroukhim R, Cai L, Brennan C, Lahti-Domenici J, Huang H et al (2004). Molecular characterization of the tumor microenvironment in breast cancer. Cancer Cell 6: 17-32.

An Z, Wang X, Geller J, Moossa AR, Hoffman RM (1998). Surgical orthotopic implantation allows high lung and lymph node metastatic expression of human prostate carcinoma cell line PC-3 in nude mice. Prostate 34: 169-74.

Anborgh PH, Mutrie JC, Tuck AB, Chambers AF (2010). Role of the metastasis-promoting protein osteopontin in the tumor microenvironment. J Cell Mol Med.

Balic M, Lin H, Young L, Hawes D, Giuliano A, McNamara G et al (2006). Most early disseminated cancer cells detected in bone marrow of breast cancer patients have a putative breast cancer stem cell phenotype. Clin Cancer Res 12: 5615-21.

Barnhart BC, Simon MC (2007). Metastasis and stem cell pathways. Cancer Metastasis Rev 26: 261-71.

Barrandon Y, Green H (1985). Cell size as a determinant of the clone-forming ability of human keratinocytes. Proc Natl Acad Sci U S A 82: 5390-4.

Barrandon Y, Green H (1987). Three clonal types of keratinocyte with different capacities for multiplication. Proc Natl Acad Sci U S A 84: 2302-6.

Basanta D, Strand DW, Lukner RB, Franco OE, Cliffel DE, Ayala GE et al (2009). The role of transforming growth factor-beta-mediated tumor-stroma interactions in prostate cancer progression: an integrative approach. Cancer Res 69: 7111-20.

Ben-Porath I, Thomson MW, Carey VJ, Ge R, Bell GW, Regev A et al (2008). An embryonic stem cell-like gene expression signature in poorly differentiated aggressive human tumors. Nat Genet 40: 499-507. 
Bhatia B, Jiang M, Suraneni M, Patrawala L, Badeaux M, Schneider-Broussard R et al (2008a). Critical and distinct roles of p16 and telomerase in regulating the proliferative life span of normal human prostate epithelial progenitor cells. J Biol Chem 283: 27957-72.

Bhatia B, Maldonado CJ, Tang S, Chandra D, Klein RD, Chopra D et al (2003). Subcellular localization and tumor-suppressive functions of 15-lipoxygenase 2 (15-LOX2) and its splice variants. J Biol Chem 278: 25091-100.

Bhatia B, Multani AS, Patrawala L, Chen X, Calhoun-Davis T, Zhou J et al (2008b). Evidence that senescent human prostate epithelial cells enhance tumorigenicity: cell fusion as a potential mechanism and inhibition by p16INK4a and hTERT. Int J Cancer 122: 1483-95.

Bhatia B, Tang S, Yang P, Doll A, Aumueller G, Newman RA et al (2005). Cell-autonomous induction of functional tumor suppressor 15-lipoxygenase 2 (15-LOX2) contributes to replicative senescence of human prostate progenitor cells. Oncogene 24: 3583-95.

Bissell MJ, Labarge MA (2005). Context, tissue plasticity, and cancer: are tumor stem cells also regulated by the microenvironment? Cancer Cell 7: 17-23.

Brown LF, Papadopoulos-Sergiou A, Berse B, Manseau EJ, Tognazzi K, Perruzzi CA et al (1994). Osteopontin expression and distribution in human carcinomas. Am J Pathol 145: 610-23.

Brown MD, Gilmore PE, Hart CA, Samuel JD, Ramani VA, George NJ et al (2007). Characterization of benign and malignant prostate epithelial Hoechst 33342 side populations. Prostate 67: 1384-96.

Campbell LL, Polyak K (2007). Breast tumor heterogeneity: cancer stem cells or clonal evolution? Cell Cycle 6: 2332-8.

Chandra D, Bratton SB, Person MD, Tian Y, Martin AG, Ayres M et al (2006). Intracellular nucleotides act as critical prosurvival factors by binding to cytochrome $\mathrm{C}$ and inhibiting apoptosome. Cell 125: 1333-46.

Chandra D, Choy G, Deng X, Bhatia B, Daniel P, Tang DG (2004). Association of active caspase 8 with the mitochondrial membrane during apoptosis: potential roles in cleaving BAP31 and caspase 3 and mediating mitochondrion-endoplasmic reticulum cross talk in etoposideinduced cell death. Mol Cell Biol 24: 6592-607.

Clarke MF, Dick JE, Dirks PB, Eaves CJ, Jamieson CH, Jones DL et al (2006). Cancer stem cells--perspectives on current status and future directions: AACR Workshop on cancer stem cells. Cancer Res 66: 9339-44. 
Clarke RB, Anderson E, Howell A, Potten CS (2003). Regulation of human breast epithelial stem cells. Cell Prolif 36 Suppl 1: 45-58.

Claudinot S, Nicolas M, Oshima H, Rochat A, Barrandon Y (2005). Long-term renewal of hair follicles from clonogenic multipotent stem cells. Proc Natl Acad Sci U S A 102: 14677-82.

Collins AT, Berry PA, Hyde C, Stower MJ, Maitland NJ (2005). Prospective identification of tumorigenic prostate cancer stem cells. Cancer Res 65: 10946-51.

Collins AT, Habib FK, Maitland NJ, Neal DE (2001). Identification and isolation of human prostate epithelial stem cells based on alpha(2)beta(1)-integrin expression. J Cell Sci 114: 386572 .

Dakhova O, Ozen M, Creighton CJ, Li R, Ayala G, Rowley D et al (2009). Global gene expression analysis of reactive stroma in prostate cancer. Clin Cancer Res 15: 3979-89.

Dalerba P, Dylla SJ, Park IK, Liu R, Wang X, Cho RW et al (2007). Phenotypic characterization of human colorectal cancer stem cells. Proc Natl Acad Sci U S A 104: 10158-63.

Dexter DL, Kowalski HM, Blazar BA, Fligiel Z, Vogel R, Heppner GH (1978). Heterogeneity of tumor cells from a single mouse mammary tumor. Cancer Res 38: 3174-81.

Dhanasekaran SM, Barrette TR, Ghosh D, Shah R, Varambally S, Kurachi K et al (2001). Delineation of prognostic biomarkers in prostate cancer. Nature 412: 822-6.

Dubrovska A, Kim S, Salamone RJ, Walker JR, Maira SM, Garcia-Echeverria C et al (2009). The role of PTEN/Akt/PI3K signaling in the maintenance and viability of prostate cancer stemlike cell populations. Proc Natl Acad Sci U S A 106: 268-73.

English HF, Santen RJ, Isaacs JT (1987). Response of glandular versus basal rat ventral prostatic epithelial cells to androgen withdrawal and replacement. Prostate 11: 229-42.

Eramo A, Lotti F, Sette G, Pilozzi E, Biffoni M, Di Virgilio A et al (2008). Identification and expansion of the tumorigenic lung cancer stem cell population. Cell Death Differ 15: 504-14.

Fang H, Harris SC, Su Z, Chen M, Qian F, Shi L et al (2009). ArrayTrack: an FDA and public genomic tool. Methods Mol Biol 563: 379-98.

Fialkow PJ, Jacobson RJ, Papayannopoulou T (1977). Chronic myelocytic leukemia: clonal origin in a stem cell common to the granulocyte, erythrocyte, platelet and monocyte/macrophage. Am J Med 63: 125-30. 
Fidler IJ (2002). The organ microenvironment and cancer metastasis. Differentiation 70: 498505.

Fidler IJ, Kim SJ, Langley RR (2007). The role of the organ microenvironment in the biology and therapy of cancer metastasis. J Cell Biochem 101: 927-36.

Fidler IJ, Talmadge JE (1986). Evidence that intravenously derived murine pulmonary melanoma metastases can originate from the expansion of a single tumor cell. Cancer Res 46: 5167-71.

Foye A, Febbo PG Cancer gene profiling in prostate cancer. Methods Mol Biol 576: 293-326.

Fukumura D, Yuan F, Monsky WL, Chen Y, Jain RK (1997). Effect of host microenvironment on the microcirculation of human colon adenocarcinoma. Am J Pathol 151: 679-88.

Ginestier C, Hur MH, Charafe-Jauffret E, Monville F, Dutcher J, Brown M et al (2007). ALDH1 is a marker of normal and malignant human mammary stem cells and a predictor of poor clinical outcome. Cell Stem Cell 1: 555-67.

Glinsky GV (2005). Death-from-cancer signatures and stem cell contribution to metastatic cancer. Cell Cycle 4: 1171-5.

Glinsky GV, Berezovska O, Glinskii AB (2005). Microarray analysis identifies a death-fromcancer signature predicting therapy failure in patients with multiple types of cancer. J Clin Invest 115: $1503-21$.

Godar S, Ince TA, Bell GW, Feldser D, Donaher JL, Bergh J et al (2008). Growth-inhibitory and tumor- suppressive functions of p53 depend on its repression of CD44 expression. Cell 134: 6273.

Gohongi T, Fukumura D, Boucher Y, Yun CO, Soff GA, Compton C et al (1999). Tumor-host interactions in the gallbladder suppress distal angiogenesis and tumor growth: involvement of transforming growth factor beta1. Nat Med 5: 1203-8.

Greaves M (2010). Cancer stem cells: back to Darwin? Semin Cancer Biol 20: 65-70.

Gu G, Yuan J, Wills M, Kasper S (2007). Prostate cancer cells with stem cell characteristics reconstitute the original human tumor in vivo. Cancer Res 67: 4807-15.

Haeno H, Levine RL, Gilliland DG, Michor F (2009). A progenitor cell origin of myeloid malignancies. Proc Natl Acad Sci U S A 106: 16616-21. 
Henderson MJ, Choi S, Beesley AH, Sutton R, Venn NC, Marshall GM et al (2008). Mechanism of relapse in pediatric acute lymphoblastic leukemia. Cell Cycle 7: 1315-20.

Heppner GH (1984). Tumor heterogeneity. Cancer Res 44: 2259-65.

Hermann PC, Huber SL, Heeschen C (2008). Metastatic cancer stem cells: a new target for anticancer therapy? Cell Cycle 7: 188-93.

Hermann PC, Huber SL, Herrler T, Aicher A, Ellwart JW, Guba M et al (2007). Distinct populations of cancer stem cells determine tumor growth and metastatic activity in human pancreatic cancer. Cell Stem Cell 1: 313-23.

Hill R, Wu H (2009). PTEN, stem cells, and cancer stem cells. J Biol Chem 284: 11755-9.

Hill RP (2006). Identifying cancer stem cells in solid tumors: case not proven. Cancer Res 66: 1891-5; discussion 1890.

Hoffman RM (2002). Whole-body fluorescence imaging with green fluorescence protein. Methods Mol Biol 183: 135-48.

Hope KJ, Jin L, Dick JE (2004). Acute myeloid leukemia originates from a hierarchy of leukemic stem cell classes that differ in self-renewal capacity. Nat Immunol 5: 738-43.

Hu M, Polyak K (2008a). Microenvironmental regulation of cancer development. Curr Opin Genet Dev 18: 27-34.

Hu M, Polyak K (2008b). Molecular characterisation of the tumour microenvironment in breast cancer. Eur J Cancer 44: 2760-5.

Hurt EM, Farrar WL (2008). Cancer stem cells: the seeds of metastasis? Mol Interv 8: 140-2.

Jantscheff P, Ziroli V, Esser N, Graeser R, Kluth J, Sukolinskaya A et al (2009). Anti-metastatic effects of liposomal gemcitabine in a human orthotopic LNCaP prostate cancer xenograft model. Clin Exp Metastasis 26: 981-92.

Jeter CR, Badeaux M, Choy G, Chandra D, Patrawala L, Liu C et al (2009). Functional evidence that the self-renewal gene NANOG regulates human tumor development. Stem Cells 27: 9931005.

Jordan CT, Guzman ML, Noble M (2006). Cancer stem cells. N Engl J Med 355: 1253-61. June CH (2007). Adoptive T cell therapy for cancer in the clinic. J Clin Invest 117: 1466-76. 
Kang Y (2009). Analysis of cancer stem cell metastasis in xenograft animal models. Methods Mol Biol 568: 7-19.

Karnoub AE, Dash AB, Vo AP, Sullivan A, Brooks MW, Bell GW et al (2007). Mesenchymal stem cells within tumour stroma promote breast cancer metastasis. Nature 449: 557-63.

Kitadai Y, Bucana CD, Ellis LM, Anzai H, Tahara E, Fidler IJ (1995). In situ mRNA hybridization technique for analysis of metastasis-related genes in human colon carcinoma cells. Am J Pathol 147: 1238-47.

Kleinman HK, Martin GR (2005). Matrigel: basement membrane matrix with biological activity. Semin Cancer Biol 15: 378-86.

Klonisch T, Wiechec E, Hombach-Klonisch S, Ande SR, Wesselborg S, Schulze-Osthoff K et al (2008). Cancer stem cell markers in common cancers - therapeutic implications. Trends Mol Med 14: $450-60$.

Kristiansen G, Pilarsky C, Pervan J, Sturzebecher B, Stephan C, Jung K et al (2004). CD24 expression is a significant predictor of PSA relapse and poor prognosis in low grade or organ confined prostate cancer. Prostate 58: 183-92.

Krivtsov AV, Twomey D, Feng Z, Stubbs MC, Wang Y, Faber J et al (2006). Transformation from committed progenitor to leukaemia stem cell initiated by MLL-AF9. Nature 442: 818-22.

Langley RR, Fidler IJ (2007). Tumor cell-organ microenvironment interactions in the pathogenesis of cancer metastasis. Endocr Rev 28: 297-321.

Lawson DA, Witte ON (2007). Stem cells in prostate cancer initiation and progression. J Clin Invest 117: 2044-50.

Leong KG, Wang BE, Johnson L, Gao WQ (2008). Generation of a prostate from a single adult stem cell. Nature 456: 804-8.

Li C, Heidt DG, Dalerba P, Burant CF, Zhang L, Adsay V et al (2007a). Identification of pancreatic cancer stem cells. Cancer Res 67: 1030-7.

Li F, Tiede B, Massague J, Kang Y (2007b). Beyond tumorigenesis: cancer stem cells in metastasis. Cell Res 17: 3-14.

Li H, Chen X, Calhoun-Davis T, Claypool K, Tang DG (2008). PC3 human prostate carcinoma cell holoclones contain self-renewing tumor-initiating cells. Cancer Res 68: 1820-5. 
Li H, Jiang M, Honorio S, Patrawala L, Jeter CR, Calhoun-Davis T et al (2009). Methodologies in assaying prostate cancer stem cells. Methods Mol Biol 568: 85-138.

Lin KK, Goodell MA (2006). Purification of hematopoietic stem cells using the side population. Methods Enzymol 420: 255-64.

Liu AY, True LD, LaTray L, Nelson PS, Ellis WJ, Vessella RL et al (1997). Cell-cell interaction in prostate gene regulation and cytodifferentiation. Proc Natl Acad Sci U S A 94: 10705-10.

Locke M, Heywood M, Fawell S, Mackenzie IC (2005). Retention of intrinsic stem cell hierarchies in carcinoma-derived cell lines. Cancer Res 65: 8944-50.

Lu X, Kang Y (2009). Cell fusion as a hidden force in tumor progression. Cancer Res 69: 8536-9.

Ma XJ, Dahiya S, Richardson E, Erlander M, Sgroi DC (2009). Gene expression profiling of the tumor microenvironment during breast cancer progression. Breast Cancer Res 11: R7.

Man YG, Gardner WA (2008). Bad seeds produce bad crops: a single stage-process of prostate tumor invasion. Int J Biol Sci 4: 246-58.

Mani SA, Guo W, Liao MJ, Eaton EN, Ayyanan A, Zhou AY et al (2008). The epithelialmesenchymal transition generates cells with properties of stem cells. Cell 133: 704-15.

Mantovani A, Allavena P, Sica A, Balkwill F (2008). Cancer-related inflammation. Nature 454: $436-44$.

Marusyk A, Polyak K (2010). Tumor heterogeneity: causes and consequences. Biochim Biophys Acta 1805: 105-17.

McAllister SS, Gifford AM, Greiner AL, Kelleher SP, Saelzler MP, Ince TA et al (2008). Systemic endocrine instigation of indolent tumor growth requires osteopontin. Cell 133: 9941005.

Miki J, Furusato B, Li H, Gu Y, Takahashi H, Egawa S et al (2007). Identification of putative stem cell markers, CD133 and CXCR4, in hTERT-immortalized primary nonmalignant and malignant tumor-derived human prostate epithelial cell lines and in prostate cancer specimens. Cancer Res 67: 3153-61.

Millikan R, Logothetis C (1997). Update of the NCCN guidelines for treatment of prostate cancer. Oncology (Williston Park) 11: 180-93.

Mills AA, Qi Y, Bradley A (2002). Conditional inactivation of p63 by Cre-mediated excision. Genesis 32: 138-41. 
Mimeault M, Batra SK (2007). Functions of tumorigenic and migrating cancer progenitor cells in cancer progression and metastasis and their therapeutic implications. Cancer Metastasis Rev 26: 203-14.

Minn AJ, Gupta GP, Siegel PM, Bos PD, Shu W, Giri DD et al (2005). Genes that mediate breast cancer metastasis to lung. Nature 436: 518-24.

Miyamoto T, Weissman IL, Akashi K (2000). AML1/ETO-expressing nonleukemic stem cells in acute myelogenous leukemia with 8;21 chromosomal translocation. Proc Natl Acad Sci U S A 97: 7521-6.

Monsky WL, Mouta Carreira C, Tsuzuki Y, Gohongi T, Fukumura D, Jain RK (2002). Role of host microenvironment in angiogenesis and microvascular functions in human breast cancer xenografts: mammary fat pad versus cranial tumors. Clin Cancer Res 8: 1008-13.

Monti M, Mancini LL, Ferrari B, Rahal D, Santoro A (2003). Complications of therapy and a diagnostic dilemma case. Case 2. Cutaneous toxicity induced by cetuximab. J Clin Oncol 21: 4651-3.

Morikawa K, Walker SM, Nakajima M, Pathak S, Jessup JM, Fidler IJ (1988). Influence of organ environment on the growth, selection, and metastasis of human colon carcinoma cells in nude mice. Cancer Res 48: 6863-71.

Morrissey C, Vessella RL (2007). The role of tumor microenvironment in prostate cancer bone metastasis. J Cell Biochem 101: 873-86.

Nakamura T, Fidler IJ, Coombes KR (2007). Gene expression profile of metastatic human pancreatic cancer cells depends on the organ microenvironment. Cancer Res 67: 139-48.

Nilsson SK, Johnston HM, Whitty GA, Williams B, Webb RJ, Denhardt DT et al (2005). Osteopontin, a key component of the hematopoietic stem cell niche and regulator of primitive hematopoietic progenitor cells. Blood 106: 1232-9.

Nowell PC (1976). The clonal evolution of tumor cell populations. Science 194: 23-8.

O'Brien CA, Pollett A, Gallinger S, Dick JE (2007). A human colon cancer cell capable of initiating tumour growth in immunodeficient mice. Nature 445: 106-10.

Odoux C, Fohrer H, Hoppo T, Guzik L, Stolz DB, Lewis DW et al (2008). A stochastic model for cancer stem cell origin in metastatic colon cancer. Cancer Res 68: 6932-41.

Oesterling JE, Martin SK, Bergstralh EJ, Lowe FC (1993). The use of prostate-specific antigen in staging patients with newly diagnosed prostate cancer. JAMA 269: 57-60. 
Padua D, Massague J (2009). Roles of TGFbeta in metastasis. Cell Res 19: 89-102.

Palapattu GS, Wu C, Silvers CR, Martin HB, Williams K, Salamone L et al (2009). Selective expression of CD44, a putative prostate cancer stem cell marker, in neuroendocrine tumor cells of human prostate cancer. Prostate 69: 787-98.

Passegue E, Jamieson CH, Ailles LE, Weissman IL (2003). Normal and leukemic hematopoiesis: are leukemias a stem cell disorder or a reacquisition of stem cell characteristics? Proc Natl Acad Sci U S A 100 Suppl 1: 11842-9.

Patocs A, Zhang L, Xu Y, Weber F, Caldes T, Mutter GL et al (2007). Breast-cancer stromal cells with TP53 mutations and nodal metastases. $N$ Engl J Med 357: 2543-51.

Patrawala L, Calhoun-Davis T, Schneider-Broussard R, Tang DG (2007). Hierarchical organization of prostate cancer cells in xenograft tumors: the CD44+alpha2beta1+ cell population is enriched in tumor-initiating cells. Cancer Res 67: 6796-805.

Patrawala L, Calhoun T, Schneider-Broussard R, Li H, Bhatia B, Tang S et al (2006). Highly purified CD44+ prostate cancer cells from xenograft human tumors are enriched in tumorigenic and metastatic progenitor cells. Oncogene 25: 1696-708.

Patrawala L, Calhoun T, Schneider-Broussard R, Zhou J, Claypool K, Tang DG (2005). Side population is enriched in tumorigenic, stem-like cancer cells, whereas ABCG2+ and ABCG2cancer cells are similarly tumorigenic. Cancer Res 65: 6207-19.

Pawelek JM, Chakraborty AK (2008). Fusion of tumour cells with bone marrow-derived cells: a unifying explanation for metastasis. Nat Rev Cancer 8: 377-86.

Pece S, Tosoni D, Confalonieri S, Mazzarol G, Vecchi M, Ronzoni S et al (2010). Biological and molecular heterogeneity of breast cancers correlates with their cancer stem cell content. Cell 140: 62-73.

Peinado H, Rafii S, Lyden D (2008). Inflammation joins the "niche". Cancer Cell 14: 347-9.

Pierce GB (1974). Neoplasms, differentiations and mutations. Am J Pathol 77: 103-118.

Polyak K (2007). Breast cancer: origins and evolution. J Clin Invest 117: 3155-63.

Polyak K, Shipitsin M, Campbell-Marrotta L, Bloushtain-Qimron N, Park SY (2009). Breast tumor heterogeneity: causes and consequences. Breast Cancer Res 11 Suppl 1: S18. 
Prince ME, Sivanandan R, Kaczorowski A, Wolf GT, Kaplan MJ, Dalerba P et al (2007). Identification of a subpopulation of cells with cancer stem cell properties in head and neck squamous cell carcinoma. Proc Natl Acad Sci U S A 104: 973-8.

Psaila B, Lyden D (2009). The metastatic niche: adapting the foreign soil. Nat Rev Cancer 9: 285-93.

Raff M (2003). Adult stem cell plasticity: fact or artifact? Annu Rev Cell Dev Biol 19: 1-22.

Ramaswamy S, Ross KN, Lander ES, Golub TR (2003). A molecular signature of metastasis in primary solid tumors. Nat Genet 33: 49-54.

Rembrink K, Romijn JC, van der Kwast TH, Rubben H, Schroder FH (1997). Orthotopic implantation of human prostate cancer cell lines: a clinically relevant animal model for metastatic prostate cancer. Prostate 31: 168-74.

Reya T, Morrison SJ, Clarke MF, Weissman IL (2001). Stem cells, cancer, and cancer stem cells. Nature 414: 105-11.

Ricci-Vitiani L, Lombardi DG, Pilozzi E, Biffoni M, Todaro M, Peschle C et al (2007). Identification and expansion of human colon-cancer-initiating cells. Nature 445: 111-5.

Richardson AM, Woodson K, Wang Y, Rodriguez-Canales J, Erickson HS, Tangrea MA et al (2007). Global expression analysis of prostate cancer-associated stroma and epithelia. Diagn Mol Pathol 16: 189-97.

Richardson GD, Robson CN, Lang SH, Neal DE, Maitland NJ, Collins AT (2004). CD133, a novel marker for human prostatic epithelial stem cells. J Cell Sci 117: 3539-45.

Rizvi AZ, Swain JR, Davies PS, Bailey AS, Decker AD, Willenbring H et al (2006). Bone marrow-derived cells fuse with normal and transformed intestinal stem cells. Proc Natl Acad Sci U S A 103: 6321-5.

Rove KO, Crawford ED (2009). Metastatic cancer in solid tumors and clinical outcome: skeletalrelated events. Oncology (Williston Park) 23: 21-7.

Sangaletti S, Di Carlo E, Gariboldi S, Miotti S, Cappetti B, Parenza M et al (2008). Macrophagederived SPARC bridges tumor cell-extracellular matrix interactions toward metastasis. Cancer Res 68: 9050-9.

Schatton T, Murphy GF, Frank NY, Yamaura K, Waaga-Gasser AM, Gasser M et al (2008). Identification of cells initiating human melanomas. Nature 451: 345-9. 
Scheel C, Onder T, Karnoub A, Weinberg RA (2007). Adaptation versus selection: the origins of metastatic behavior. Cancer Res 67: 11476-9; discussion 11479-80.

Sell S, Pierce GB (1994). Maturation arrest of stem cell differentiation is a common pathway for the cellular origin of teratocarcinomas and epithelial cancers. Lab Invest 70: 6-22.

Sgroi DC (2010). Preinvasive breast cancer. Annu Rev Pathol 5: 193-221.

Shackleton M, Quintana E, Fearon ER, Morrison SJ (2009). Heterogeneity in cancer: cancer stem cells versus clonal evolution. Cell 138: 822-9.

Shen MM, Wang X, Economides KD, Walker D, Abate-Shen C (2008). Progenitor cells for the prostate epithelium: roles in development, regeneration, and cancer. Cold Spring Harb Symp Quant Biol 73: 529-38.

Sheridan C, Kishimoto H, Fuchs RK, Mehrotra S, Bhat-Nakshatri P, Turner CH et al (2006). CD44+/CD24- breast cancer cells exhibit enhanced invasive properties: an early step necessary for metastasis. Breast Cancer Res 8: R59.

Shipitsin M, Campbell LL, Argani P, Weremowicz S, Bloushtain-Qimron N, Yao J et al (2007). Molecular definition of breast tumor heterogeneity. Cancer Cell 11: 259-73.

Shmelkov SV, Butler JM, Hooper AT, Hormigo A, Kushner J, Milde T et al (2008). CD133 expression is not restricted to stem cells, and both CD133+ and CD133-metastatic colon cancer cells initiate tumors. J Clin Invest 118: 2111-20.

Singh D, Febbo PG, Ross K, Jackson DG, Manola J, Ladd C et al (2002). Gene expression correlates of clinical prostate cancer behavior. Cancer Cell 1: 203-9.

Singh SK, Clarke ID, Terasaki M, Bonn VE, Hawkins C, Squire J et al (2003). Identification of a cancer stem cell in human brain tumors. Cancer Res 63: 5821-8.

Singh SK, Hawkins C, Clarke ID, Squire JA, Bayani J, Hide T et al (2004). Identification of human brain tumour initiating cells. Nature 432: 396-401.

Sneddon JB, Werb Z (2007). Location, location, location: the cancer stem cell niche. Cell Stem Cell 1: 607-11.

Staack A, Donjacour AA, Brody J, Cunha GR, Carroll P (2003). Mouse urogenital development: a practical approach. Differentiation 71: 402-13.

Steeg PS (2006). Tumor metastasis: mechanistic insights and clinical challenges. Nat Med 12: 895-904. 
Stephenson RA, Dinney CP, Gohji K, Ordonez NG, Killion JJ, Fidler IJ (1992). Metastatic model for human prostate cancer using orthotopic implantation in nude mice. J Natl Cancer Inst 84: 951-7.

Tang DG, Patrawala L, Calhoun T, Bhatia B, Choy G, Schneider-Broussard R et al (2007). Prostate cancer stem/progenitor cells: identification, characterization, and implications. Mol Carcinog 46: 1-14.

Todaro M, Alea MP, Di Stefano AB, Cammareri P, Vermeulen L, Iovino F et al (2007). Colon cancer stem cells dictate tumor growth and resist cell death by production of interleukin-4. Cell Stem Cell 1: 389-402.

Tomlins SA, Mehra R, Rhodes DR, Cao X, Wang L, Dhanasekaran SM et al (2007). Integrative molecular concept modeling of prostate cancer progression. Nat Genet 39: 41-51.

True L, Coleman I, Hawley S, Huang CY, Gifford D, Coleman R et al (2006). A molecular correlate to the Gleason grading system for prostate adenocarcinoma. Proc Natl Acad Sci U S A 103: 10991-6.

Tsingotjidou AS, Zotalis G, Jackson KR, Sawyers C, Puzas JE, Hicks DG et al (2001). Development of an animal model for prostate cancer cell metastasis to adult human bone. Anticancer Res 21: 971-8.

Tsujimura A, Koikawa Y, Salm S, Takao T, Coetzee S, Moscatelli D et al (2002). Proximal location of mouse prostate epithelial stem cells: a model of prostatic homeostasis. J Cell Biol 157: 1257-65.

Tsuzuki Y, Mouta Carreira C, Bockhorn M, Xu L, Jain RK, Fukumura D (2001). Pancreas microenvironment promotes VEGF expression and tumor growth: novel window models for pancreatic tumor angiogenesis and microcirculation. Lab Invest 81: 1439-51.

Tu SM, Lin SH, Logothetis CJ (2002). Stem-cell origin of metastasis and heterogeneity in solid tumours. Lancet Oncol 3: 508-13.

Tuck AB, Chambers AF, Allan AL (2007). Osteopontin overexpression in breast cancer: knowledge gained and possible implications for clinical management. J Cell Biochem 102: 85968 .

van 't Veer LJ, Dai H, van de Vijver MJ, He YD, Hart AA, Mao M et al (2002). Gene expression profiling predicts clinical outcome of breast cancer. Nature 415: 530-6.

van de Vijver MJ, He YD, van't Veer LJ, Dai H, Hart AA, Voskuil DW et al (2002). A geneexpression signature as a predictor of survival in breast cancer. N Engl J Med 347: 1999-2009. 
van den Berg DL, Snoek T, Mullin NP, Yates A, Bezstarosti K, Demmers J et al An Oct4centered protein interaction network in embryonic stem cells. Cell Stem Cell 6: 369-81.

van Weerden WM, Romijn JC (2000). Use of nude mouse xenograft models in prostate cancer research. Prostate 43: 263-71.

Varambally S, Yu J, Laxman B, Rhodes DR, Mehra R, Tomlins SA et al (2005). Integrative genomic and proteomic analysis of prostate cancer reveals signatures of metastatic progression. Cancer Cell 8: 393-406.

Vera-Ramirez L, Sanchez-Rovira P, Ramirez-Tortosa CL, Quiles JL, Ramirez-Tortosa MC, Alvarez JC et al (2010). Gene-expression profiles, tumor microenvironment, and cancer stem cells in breast cancer: Latest advances towards an integrated approach. Cancer Treat Rev.

Verhulst A, Persy VP, Van Rompay AR, Verstrepen WA, Helbert MF, De Broe ME (2002). Osteopontin synthesis and localization along the human nephron. J Am Soc Nephrol 13: 1210-8.

Vermeulen L, Todaro M, de Sousa Mello F, Sprick MR, Kemper K, Perez Alea M et al (2008). Single-cell cloning of colon cancer stem cells reveals a multi-lineage differentiation capacity. Proc Natl Acad Sci U S A 105: 13427-32.

Wang JC (2007). Evaluating therapeutic efficacy against cancer stem cells: new challenges posed by a new paradigm. Cell Stem Cell 1: 497-501.

Wang JC (2010). Good cells gone bad: the cellular origins of cancer. Trends Mol Med 16: 14551 .

Wang X, Kruithof-de Julio M, Economides KD, Walker D, Yu H, Halili MV et al (2009). A luminal epithelial stem cell that is a cell of origin for prostate cancer. Nature 461: 495-500.

Wang Y, Revelo MP, Sudilovsky D, Cao M, Chen WG, Goetz L et al (2005a). Development and characterization of efficient xenograft models for benign and malignant human prostate tissue. Prostate 64: 149-59.

Wang Y, Xue H, Cutz JC, Bayani J, Mawji NR, Chen WG et al (2005b). An orthotopic metastatic prostate cancer model in SCID mice via grafting of a transplantable human prostate tumor line. Lab Invest 85: 1392-404.

Weber GF, Ashkar S, Glimcher MJ, Cantor H (1996). Receptor-ligand interaction between CD44 and osteopontin (Eta-1). Science 271: 509-12.

Weigelt B, Peterse JL, van 't Veer LJ (2005). Breast cancer metastasis: markers and models. Nat Rev Cancer 5: 591-602. 
Weiss L (2000). Metastasis of cancer: a conceptual history from antiquity to the 1990s. Cancer Metastasis Rev 19: I-XI, 193-383.

Weissman IL (2000). Stem cells: units of development, units of regeneration, and units in evolution. Cell 100: 157-68.

Welsh JB, Sapinoso LM, Su AI, Kern SG, Wang-Rodriguez J, Moskaluk CA et al (2001). Analysis of gene expression identifies candidate markers and pharmacological targets in prostate cancer. Cancer Res 61: 5974-8.

Werbowetski-Ogilvie TE, Bhatia M (2008). Pluripotent human stem cell lines: what we can learn about cancer initiation. Trends Mol Med 14: 323-32.

Wicha MS (2006). Cancer stem cells and metastasis: lethal seeds. Clin Cancer Res 12: 5606-7.

Yang M, Jiang P, Sun FX, Hasegawa S, Baranov E, Chishima T et al (1999). A fluorescent orthotopic bone metastasis model of human prostate cancer. Cancer Res 59: 781-6.

Yang ZF, Ho DW, Ng MN, Lau CK, Yu WC, Ngai P et al (2008). Significance of CD90+ cancer stem cells in human liver cancer. Cancer Cell 13: 153-66.

Yeung TM, Gandhi SC, Wilding JL, Muschel R, Bodmer WF Cancer stem cells from colorectal cancer-derived cell lines. Proc Natl Acad Sci U S A 107: 3722-7.

Yu F, Yao H, Zhu P, Zhang X, Pan Q, Gong C et al (2007). let-7 regulates self renewal and tumorigenicity of breast cancer cells. Cell 131: 1109-23.

Zaehres H, Lensch MW, Daheron L, Stewart SA, Itskovitz-Eldor J, Daley GQ (2005). Highefficiency RNA interference in human embryonic stem cells. Stem Cells 23: 299-305.

Zhang HB, Ren CP, Yang XY, Wang L, Li H, Zhao M et al (2007). Identification of labelretaining cells in nasopharyngeal epithelia and nasopharyngeal carcinoma tissues. Histochem Cell Biol 127: 347-54.

Zhou JR, Yu L, Zhong Y, Nassr RL, Franke AA, Gaston SM et al (2002). Inhibition of orthotopic growth and metastasis of androgen-sensitive human prostate tumors in mice by bioactive soybean components. Prostate 53: 143-53. 


\section{VITA}

Hangwen Li was born in Anyang, Henan, P.R China on March 27, 1974, the son of Zhengguo Li and Meiying Wang. After completing high school in Anyang No 1 High School, Anyang, Henan, China, in 1991, he entered the Medical School of Zhengzhou University, Zhengzhou, Henan, China and received the degree of Bachelor of Medicine in 1996. From 19962000, he was employed as a physician in Anyang CDC, Anyang, Henan, China, P.R. China. From 2000-2003, he entered the Graduate School of China CDC, Beijing, China and attained the degree of Master of Medicine in 2000. In fall 2003, Mr. Li entered the Graduate School of The University of Texas at Austin.

Permanent email: lihangwen@yahoo.com

This dissertation was typed by the author. 